\title{
Stoßinduzierte Strömungsablösung in transitionellen Grenzschichten
}

\author{
Dissertation \\ zur Erlangung des mathematisch-naturwissenschaftlichen Doktorgrades \\ "Doctor rerum naturalium" \\ der Georg-August-Universität Göttingen \\ im Promotionsstudiengang Physik \\ der Georg-August University School of Science (GAUSS) \\ vorgelegt von

\section{Jens Bernard Lunte} \\ aus Lohne
}

Göttingen, 2021 


\section{Betreuungsausschuss:}

\section{Prof. Dr. Dr. Andreas Dillmann}

Georg-August-Universität Göttingen, Institut für Dynamik komplexer Systeme und

Deutsches Zentrum für Luft- und Raumfahrt e.V., Institut für Aerodynamik und Strömungstechnik

Prof. Dr. Martin Rein

Georg-August-Universität Göttingen, Institut für Dynamik komplexer Systeme und

Deutsches Zentrum für Luft- und Raumfahrt e.V., Institut für Aerodynamik und Strömungstechnik

\section{Dr. Erich Schülein}

Deutsches Zentrum für Luft- und Raumfahrt e.V., Institut für Aerodynamik und Strömungstechnik

\section{Mitglieder der Prüfungskommission:}

\section{Referent: Prof. Dr. Dr. Andreas Dillmann}

Georg-August-Universität Göttingen, Institut für Dynamik komplexer Systeme und

Deutsches Zentrum für Luft- und Raumfahrt e.V., Institut für Aerodynamik und Strömungstechnik

\section{Korreferent: Prof. Dr. Martin Rein}

Georg-August-Universität Göttingen, Institut für Dynamik komplexer Systeme und

Deutsches Zentrum für Luft- und Raumfahrt e.V., Institut für Aerodynamik und Strömungstechnik

\section{Weitere Mitglieder der Prüfungskommission:}

Prof. Dr. Andreas Tilgner,

Georg-August-Universität Göttingen, Institut für Geophysik

Prof. Dr. Wolfgang Glatzel,

Georg-August-Universität Göttingen, Institut für Astrophysik

Prof. Dr. Ulrich Parlitz,

Georg-August-Universität Göttingen, Institut für Dynamik komplexer Systeme und

Max-Planck-Institut für Dynamik und Selbstorganisation

\section{Prof. Dr. Stefan Klumpp,}

Georg-August-Universität Göttingen, Institut für Dynamik komplexer Systeme

Tag der mündlichen Prüfung: 02.12.2021 


\section{Kurzbeschreibung}

Die Auswirkungen von transitionellen Stoß-Grenzschicht-Wechselwirkungen (SGWW) auf die induzierten Wärmelasten und den Verlauf des laminar-turbulenten Umschlags sind Thema dieser Arbeit. Experimentelle Untersuchungen wurden bei Mach 6 am Rohrwindkanal Göttingen an einer ebenen Platte durchgeführt. Die Strömungstopologie wurde mittels Schattenaufnahmen untersucht und die Wärmestromdichte über quantitative Infrarotthermographie bestimmt. Zusätzlich wurden die Druck- und Dichteschwankungen innerhalb der Grenzschicht mittels Drucksensoren bzw. fokussierter Laser-Differentialinterferometrie detektiert. Verschiedene Verfahren zur Quantifizierung des Transitionsfortschrittes wurden anhand der Messdaten verglichen und lieferten systematische Abweichungen in der Intermittenzverteilung. Die Abweichungen ließen sich anhand der experimentellen Implementierung erklären und liegen im Rahmen der Messgenauigkeit.

Unter Verwendung eines zweidimensionalen einfallenden Stoßes wurde systematisch und unabhängig die Stoßauftreffposition $\left(x_{\text {imp }}\right)$, die Stoßintensität sowie die Lage der natürlichen Transition variiert. Die Messungen zeigen einen systematischen Einfluss der variierten Parameter auf die räumliche Verteilung der Wärmestromdichte. Hierbei korreliert die maximale stoßinduzierte dimensionslose Wärmestromdichte $S t_{\max }$ mit dem Transitionsende der Grenzschicht. Die Entfernung bis zum Transitionsende und der Wert von $S t_{\max }$ hängen maßgeblich von der Kombination aus Stoßintensität und dem Grenzschichtzustand bei $x_{\text {imp }}$ ab. Durch eine Skalierung der relativen Lage der Stoßauftreffposition mit der Länge des ungestörten Transitionsgebietes ergibt sich eine einheitliche Verteilung der normierten $S t_{\text {max }}$-Werte. Für die Normierung wird zum einen der Einfluss der Stoßintensität mit dem Skalierungsgesetz von Holden (1977) berücksichtigt und zum anderen ein aus der ungestörten St-Verteilung abgeleiteter Referenzwert verwendet. Die höchsten Wärmestromverstärkungen werden bei transitionellen SGWW beobachtet.

\section{Abstract}

The effects of transitional shock-wave/boundary-layer interactions (SWBLI) on the induced heat loads and the development of the laminar-turbulent transition are the subject of this work. Experimental investigations were conducted in the Ludwieg-Tube Facility at DLR Göttingen at Mach 6 on a flat plate. The flow topology was investigated by shadowgraphy and the heat flux density was determined via quantitative infrared thermography. Additionally, the pressure and density fluctuations within the boundary layer were detected using pressure sensors and focused laser differential interferometry, respectively. Different methods for quantifying the transition progress were compared and revealed systematic deviations in the intermittency distribution. The discrepancies could be explained by the experimental implementation and are within the accuracy of the measurements.

Using a two-dimensional impinging shock, the impingement position $\left(x_{\mathrm{imp}}\right)$, the shock intensity, and the location of the natural transition were systematically and indepen- 
dently varied. The measurements show a systematic influence of the varied parameters on the heat flux distribution. In this context, the maximum shock-induced dimensionless heat flux density $S t_{\max }$ correlates with the transition end of the boundary layer. The length until the transition completes and the value of $S t_{\max }$ depend critically on the combination of the shock intensity and the boundary layer state at $x_{\mathrm{imp}}$. Scaling the relative position of the shock impingement point with the length of the undisturbed transition region yields a uniform distribution of the normalized $S t_{\max }$ values. For normalization, the influence of the shock intensity is taken into account with the scaling law of Holden (1977) and a reference value derived from the undisturbed St-distribution is used. The highest heat transfer amplifications are observed in transitional SGWW. 


\section{Vorwort}

Die vorliegende Dissertation ist das Ergebnis meiner Arbeit als Doktorand und wissenschaftlicher Mitarbeiter in der Abteilung Hochgeschwindigkeitskonfigurationen des Instituts für Aerodynamik und Strömungstechnik des Deutschen Zentrums für Luftund Raumfahrt (DLR) in Göttingen.

Eine Promotion kann nicht alleine erstellt werden. Daher widme ich diesen Abschnitt den Menschen, die mir geholfen haben, diese Arbeit zu verwirklichen.

Zunächst möchte ich mich bei Herrn Prof. Dr. Dr. Andreas Dillmann und Herrn Prof. Dr. Martin Rein für die Übernahme des Referates und Korreferates dieser Dissertation bedanken.

Vielen Dank an die gesamte Abteilung Hochgeschwindigkeitskonfigurationen für die angenehme Arbeitsatmosphäre und Hilfsbereitschaft. Insbesondere möchte ich mich bei der Flugkörpergruppe für die Einarbeitung, die Unterstützung und die vielen Diskussionen bedanken. Hervorheben möchte ich meinen fachlichen Betreuer, Dr. Erich Schülein, der sich immer die Zeit nahm Probleme zu diskutieren und Lösungen zu erarbeiten.

Mein Dank gilt auch denen, die mich bei der technischen Umsetzung der Experimente unterstützt haben. Dazu gehören Uwe Becker mit der Konstruktion der Windkanalmodelle, Karl Borchert und Lothar Schuster mit der Modellvorbereitung und Realisierung am Windkanal. Hartmut Haselmeyer mit der Unterstützung bei der Messtechnik sowie Karsten Pfeiffer für den Betrieb des Windkanals und die Hilfe während der Messkampagnen.

Abschließend möchte ich mich noch bei meinen Eltern bedanken, die mir mein Studium ermöglicht haben und mir während der Corona-Einschränkungen einen hervorragenden Heimarbeitsplatz zur Verfügung stellten. 



\section{Inhaltsverzeichnis}

1 Einleitung 1

1.1 Zielsetzung und Gliederung $\ldots \ldots \ldots \ldots \ldots$

$\begin{array}{lll}2 & \text { Theorie } & 7\end{array}$

2.1 Grenzschichten in kompressiblen Strömungen $\ldots \ldots \ldots \ldots \ldots$

2.1 .1 Laminare Grenzschicht . . . . . . . . . . . . . . . . . . . . . . 7

2.1 .2 Turbulente Grenzschicht . . . . . . . . . . . . . . . . . . 11

2.1 .3 Transition . . . . . . . . . . . . . . . . . . . . . . . . . 13

2.2 Stoß-Grenzschicht-Wechselwirkung $\ldots \ldots \ldots \ldots \ldots$

$2.2 .1 \quad$ Schwache SGWW $\ldots \ldots \ldots \ldots \ldots$

2.2 .2 Starke SGWW . . . . . . . . . . . . . . . . . . . 20

2.2 .3 Grenzschichtzustand bei SGWW . . . . . . . . . . . 22

3 Messaufbau 25

3.1 Rohrwindkanal Göttingen . . . . . . . . . . . . . . . . . . 25

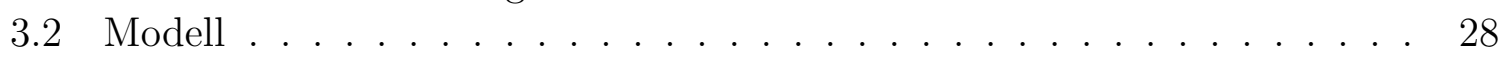

3.3 Schattenverfahren . . . . . . . . . . . . . . . . . . . . . . 30

3.4 Quantitative Infrarotthermographie . . . . . . . . . . . . . . . . . 31

3.5 Zeitaufgelöste Messungen $\ldots \ldots \ldots \ldots \ldots$

3.5 .1 Druckmessungen . . . . . . . . . . . . . . . . . 37

3.5.2 Fokussierte Laser-Differentialinterferometrie . . . . . . . . . . . 41

3.6 Messmatrix . . . . . . . . . . . . . . . . . . . . . . 52

4 Ergebnisse und Diskussion $\quad 53$

4.1 Ungestörte Grenzschicht . . . . . . . . . . . . . . . . . 53

4.1 .1 Schattenaufnahmen . . . . . . . . . . . . . . . . 53

4.1 .2 Verteilung der Wärmestromdichte . . . . . . . . . . . . . . 55

4.1 .3 Druckschwankungen $\ldots \ldots \ldots \ldots \ldots$

4.1 .4 Dichteschwankungen . . . . . . . . . . . . . . . . . . . . 64

4.1 .5 Intermittenz . . . . . . . . . . . . . . . . . . . . . 72

4.2 Stoß-Grenzschicht-Interaktion . . . . . . . . . . . . . . . . . 81

4.2 .1 Schattenaufnahmen . . . . . . . . . . . . . . . . . . . 82

4.2 .2 Stoßinduzierte Wärmestromdichte . . . . . . . . . . . . . . 86

4.2 .3 Intermittenzverlauf bei SGWW . . . . . . . . . . . . . . . 94

4.2 .4 LDS bei SGWW $\ldots \ldots \ldots \ldots \ldots$

$4.2 .5 \quad$ Skalierungsgesetze für kritische Kenngrößen $\ldots \ldots \ldots$ 
$\begin{array}{lll}5 & \text { Zusammenfassung } & 115\end{array}$

\begin{tabular}{ll}
\hline A Anhang & 117
\end{tabular}

A.1 Herleitung der Gleichungen zur kompressiblen laminaren Grenzschicht . . 117

A.2 Informationen bezüglich der PCB-Sensoren . . . . . . . . . . . . . . . 119

A.3 Tabellen zur Messmatrix . . . . . . . . . . . . . . . . . . . . . . . . . . . 121

A.4 Werte zur Intermittenzbestimmung . . . . . . . . . . . . . . . 122 


\section{Symbolverzeichnis}

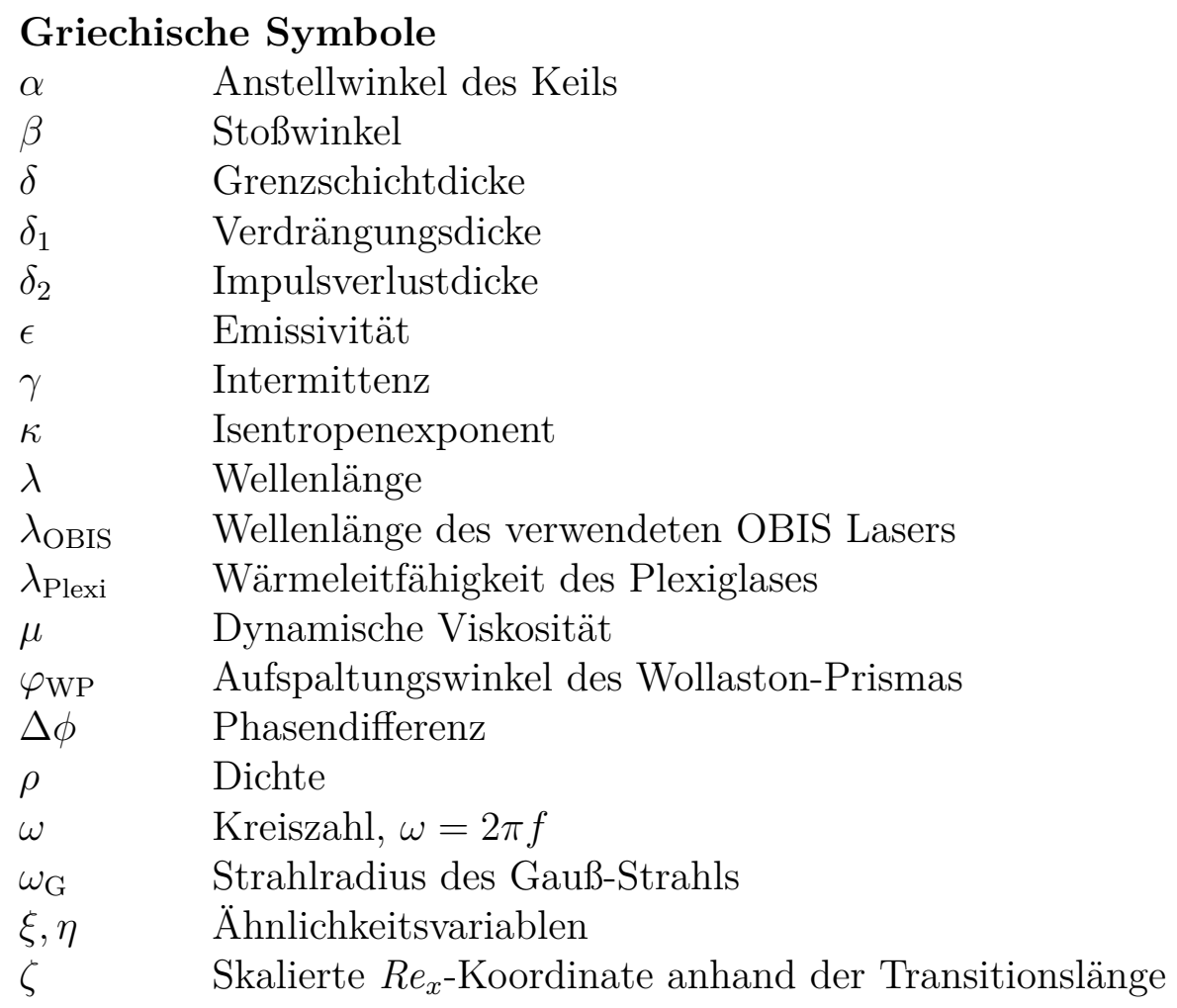

\section{Lateinische Symbole}

$c_{f} \quad$ Reibungsbeiwert

$c_{p} \quad$ Spezifische Wärmekapazität bei konstantem Druck

$f \quad$ Frequenz

$f_{\mathrm{L}} \quad$ Brennweite von Linsen

$h \quad$ Spezifische Enthalpie

I Intensität

$K_{\mathrm{GD}} \quad$ Gladstone-Dale-Konstante

$k \quad$ Wellenzahl, $k=2 \pi / \lambda$

Ma Machzahl

$\operatorname{Pr} \quad$ Prandtl-Zahl

$p \quad$ Druck

$\dot{q}_{\mathrm{w}} \quad$ Wärmestromdichte an der Modellwand

$r \quad$ Radius

$r \quad$ Rückgewinnfaktor 


$\begin{array}{ll}R & \text { Spezifische Gaskonstante } \\ R_{\mathrm{A}} & \text { Abschlusswiderstand } \\ R e & \text { Reynolds-Zahl } \\ R e_{1} & \text { Einheitsreynoldszahl, } R e_{1}=\rho_{\infty} u_{\infty} / \mu_{\infty} \\ R_{z} & \text { Oberflächenrauhigkeit } \\ S t & \text { Stanton-Zahl } \\ \Delta s_{\mathrm{opt}} & \text { Optische Wegdifferenz } \\ t & \text { Zeit } \\ U & \text { Spannung } \\ u & \text { Strömungsgeschwindigkeit } \\ u_{\mathrm{Ph}} & \text { Phasengeschwindigkeit } \\ x, y, z & \text { räumliche Koordinaten }\end{array}$

\section{Tiefgestellte Indizes}

0 Ruhegröße

1 Strömungsgebiet stromauf des einfallenden Stoßes

2 Strömungsgebiet zwischen dem einfallenden und dem reflektierten Stoß

$3 \quad$ Strömungsgebiet stromab des reflektierten Stoßes

ui $\quad$ Wert an der Stelle der Stromaufwirkung

aw Annahme einer adiabatischen Wand

imp Größe an der nicht-viskosen Stoßauftreffposition

lami Laminare Grenzschichtbedingung

Plexi Wert des verwendeten Plexiglases

$\max \quad$ Wert am Maximum

S Größe einer hypothetischen Störung

$\mathrm{Tb} \quad$ Größe am Transitionsbeginn

turb Turbulente Grenzschichtbedingung

Te Größe am Transitionsende

$\infty \quad$ Anströmbedingungen

w $\quad$ Werte an der Modellwand

Abkürzungen

FLDI Fokussierte Laser-Differentialinterferometrie

LDS Leistungsdichtespektrum

PCB PCB-132A31 Sensoren der Firma PCB Synotech GmbH

QIRT Quantitative Infrarotthermographie

SGWW Stoß-Grenzschicht-Wechselwirkung 


\section{Abbildungsverzeichnis}

1.1 Beispiele von SGWW am Überschallfluggerät (Babinsky \& Harvey |2011) 1

2.1 Grenzschichtprofile der Ähnlichkeitslösungen einer kompressiblen laminaren Grenzschicht für repräsentative Strömungsbedingungen am RWG . . 9

$2.2 \quad$ Flussdiagramm bezüglich der verschiedenen Pfade zur Transition (Abbildung angepasst aus $($ Fedorov $\mid 2011))] \ldots \ldots \ldots$. . . . . . . . . . 14

$2.3 \quad$ Skizze von möglichen externen Störungen, die auf eine laminare Grenzschicht im Überschall wirken (übersetzt aus (Zhong \& Wang $\mid$ 2012)) $\ldots$. . . 14

2.4 Einfluss der Anströmmachzahl auf die zeitlichen Verstärkungsraten der ersten und zweiten Störungsmode. Angegeben ist die Verstärkungsrate für den instabilsten Ausbreitungswinkel der Welle (nachgezeichnet aus $(\operatorname{Mack} 1984))$. . . . . . . . . . . . . . . . . . . . 16

2.5 Görtler-Wirbel innerhalb der Grenzschicht entlang einer konkaven Wand

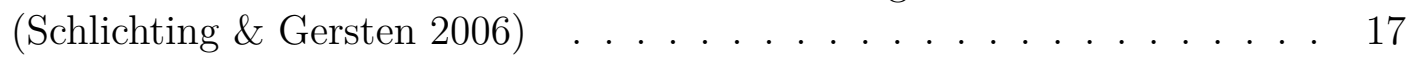

2.6 Grundlegende Konfigurationen für 2D SGWW (nicht maßstabsgetreu) . . 19

$2.7 \quad$ Strömungsstruktur eines einfallenden Stoßes ohne Ablösung . . . . . . . 20

2.8 Strömungsstruktur eines einfallenden Stoßes mit Ablösung (übersetzt aus Babinsky \& Harvey $(2011))$. . . . . . . . . . . . . . . . . . 21

2.9 Druckverteilungen auf der Modelloberfläche aufgrund einfallender SGWW 22

3.1 Skizze der Ludwieg-Rohr Anlage RWG am DLR Göttingen (Schülein 1999) 25

3.2 Über die Messzeit sind der Totaldruck und die Totaltemperatur stromauf der Lavaldüse dargestellt. Zusätzlich ist die daraus berechnete Einheitsreynoldszahl in der Messstrecke aufgetragen. Die Kurven sind aus 44 einzelnen Versuchen gemittelt und die jeweilige Streuung in Form der $1 \sigma$-Umgebung durch gestrichelte Linien angezeigt. . . . . . . . . . . . . 27

3.3 Skizze des Windkanalmodells bestehend aus einer ebenen Platte mit montiertem Stoßgenerator. In der Aussparung ist der schwarze PlexiglasEinsatz dargestellt . . . . . . . . . . . . . . . . . . . . 28

3.4 Vereinfachtes Stoßsystem des Modells. Die Skizze zeigt sowohl den gewünschten Verlauf (grün) des Vorderkantenstoßes als auch einen unerwünschten Verlauf (rot) mit der Stoßreflexion an der Unterseite des Stoßgenerators . . . . . . . . . . . . . . . . . . . . 29

$3.5 \quad$ Skizze des Messaufbaus der fokussierten Schattenaufnahmen am RWG. . 30 
3.6 Beispiel für die Auswertung einer Schattenaufnahme. Markiert sind die beiden Referenzpunkte (rote Punkte) für die Umrechnung ins Koordinatensystem der ebenen Platte und die Lage des Verdichtungsstoßes samt

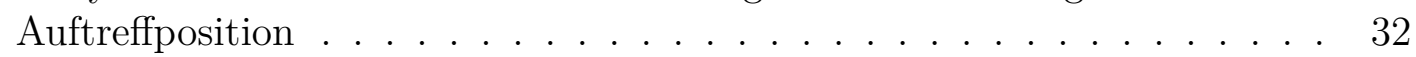

3.7 Skizze der QIRT Messung (angepasst aus (Schülein||2014)). . . . . . . . . . 33

3.8 Beispiel für die gemessene Temperaturverteilung mit SGWW und die Bedienoberfläche von dem Programm HeatFit . . . . . . . . . . . . . . . . 34

$3.9 \quad$ Realteil des gewählten Mutter-Wavelet mit $\omega_{0}=4 \pi \ldots$. . . . . . . . . . . 36

3.10 Zeichnungen zum Einbau der PCB-Sensoren . . . . . . . . . . . . . . . . 37

3.11 Schwingungsübertragung der PCB-Sensoren für unterschiedliche Einbauvarianten . . . . . . . . . . . . . . . . . . . . 39

3.12 LDS eines PCB-Sensors . . . . . . . . . . . . . . . . . . 40

3.13 Skizze des FLDI Aufbaus. Der aufgeteilte Strahl ist blau und rot dargestellt. Überlappende Bereiche sind violett . . . . . . . . . . . . . . . . . . 41

3.14 Skizze vom Strahlengang beim klassischen FLDI-Aufbau und bei der Verwendung von Zylinderlinsen (CFLDI). Beim klassischen FLDI wird an ebenen Geometrien ein Großteil des Strahldurchmessers abgeschattet. Um der Abschattung zu entgehen, kann ein Glas in die Platte eingebaut werden, wobei der Strahlengang senkrecht zur Oberfläche verläuft. . . . . . . . 43

3.15 Schnitt durch das Plattenmodell samt Schacht. Der Schacht beginnt an der Unterseite der ebenen Platte und endet an der Wand des Windkanals. Durch den Schacht wird der Strahlenverlauf des FLDI-Aufbaus vollständig von der Strömung unterhalb der ebenen Platte isoliert. . . . . . . . . . . . 44

3.16 Skalierte Intensitätsverteilungen der Laserstrahlprofile beim FLDIAufbau an mehreren Positionen entlang der optischen Achse . . . . . . . 46

3.17 Antwortverhalten der FLDI-Technik auf eine idealisierte eindimensionale Dichtestörung. Die messbare Signalamplitude ist in a) zusammen mit der Signalamplitude eines idealen Differentialinterferometers dargestellt. Die Systemfunktion aufgrund des endlichen Abstandes der Fokuspunkte ist in b) für einen infinitesimalen und einen endlichen Fokusradius dargestellt. . 49

3.18 Effektivwerte der Freistrahl-Messung . . . . . . . . . . . . 50

3.19 LDS des Freistrahls an verschiedenen Positionen entlang der optischen Achse auf der Empfängerseite des FLDI-Aufbaus. Die Reduzierung des LDS mit zunehmender Entfernung vom Fokuspunkt belegt die reduzierte Empfindlichkeit der FLDI-Technik außerhalb der Fokusebene . . . . . . . 51

4.1 Über jeweils 200 Einzelaufnahmen gemittelte Schattenaufnahmen von der Umströmung der ebenen Platte für zwei $R e_{1} \ldots \ldots$. . . . . . . . . . . . 54

4.2 Oberflächenverteilung der Stantonzahl. Das Einsetzen der Transition ist anhand der steigenden Wärmestromdichte erkennbar. Zusätzlich ist eine keilförmige Struktur zwischen $x \approx 150 \mathrm{~mm}-325 \mathrm{~mm}$ erkennbar. Die dargestellte Verteilung gehört zu Plattenposition B und $R e_{1}=24 \cdot 10^{6} \mathrm{~m}^{-1} .55$

4.3 Spannweitig gemittelte Stantonzahlverteilung der natürlichen Transition entlang der ebenen Platte für verschiedene $R e_{1} \ldots$. . . . . . . . . . . . 57 
4.4 Visualisierung der Transitionslage bei $R e_{1}=14 \cdot 10^{6} \mathrm{~m}^{-1}$ anhand der StVerteilung und der PCB-Sensorposition (a). Die skalierten Zeitsignale der dazugehörigen PCB-Sensoren zeigen die Turbulenzflecken innerhalb der Grenzschicht bei unterschiedlichen Transitionszuständen (b). Die Auswirkungen der Transition auf das Frequenzspektrum der Druckschwankungen zeigen die LDS (c) . . . . . . . . . . . . . . . . . . . . . . . . . 59

4.5 LDS der Druckschwankungen in einer turbulenten Grenzschicht $(x=$ $473.3 \mathrm{~mm}$ bei $R e_{1}=24 \cdot 10^{6} \mathrm{~m}^{-1}$ ). Dazu ist die Frequenzantwort der PCB-Sensoren innerhalb einer turbulenten Grenzschicht basierend auf den Rechnungen von Corcos $(1963)$ dargestellt. . . . . . . . . . . . . . . . . . 61

4.6 Zur Übersichtlichkeit wurde der zeitlicher Verlauf der Druckschwankungen bei unterschiedlichen $R e_{1}$ zueinander versetzt (a). Die LDS verdeutlichen wie sich der Transitionszustand an einer festen Messposition aufgrund der $R e_{1}$ verändert (b). . . . . . . . . . . . . . . . . . . . . 62

4.7 Zeitlicher Verlauf der Druckschwankungen bei unterschiedlichen $\operatorname{Re}_{1}$ (a) und die dazugehörigen Wavelet-Transformationen (b-e). . . . . . . . . . . 63

4.8 Mittels FLDI gemessene Leistungsdichtesprektren der Anströmung für mehrere $R e_{1} \ldots \ldots \ldots \ldots \ldots \ldots \ldots$. . . . . . . . . . . . . . . . . . . . . .

4.9 Leistungsdichtespektren der Dichteschwankungen in der Grenzschicht an verschiedenen $x$-Positionen für zwei unterschiedliche $R e_{1}$. Die Spektren zeigen die Veränderung aufgrund des einsetzenden laminar-turbulenten Umschlags . . . . . . . . . . . . . . . . . . . 67

4.10 Zeitlicher Verlauf (a) der FLDI-Messung an der Position $x=393.6 \mathrm{~mm}$ bei $R e_{1}=14 \cdot 10^{6} \mathrm{~m}^{-1}$ und die dazugehörige Wavelet-Transformation (b). Der bandpassgefilterte Ausschnitt (c) von einer zweiten Mack-Mode ähnelt einem Wellenpaket. . . . . . . . . . . . . . . . . . . . 69

4.11 Bei einem etablierten Skalierungsgesetz (a) für die Frequenz der zweiten Mack-Mode $f_{\text {Mack }}$ verbleibt eine Abhängigkeit bezüglich $R e_{1}(\mathrm{~b})$. Durch die Verwendung einer mechanismusbasierten Längenskala $z_{\text {inf }}$ können die $R e_{1}$-Effekte bei der Skalierung adäquat berücksichtigt werden (c,d). . . . 71

4.12 Darstellung der Zwischenschritte bei der Bestimmung von $\gamma$ mittels Detektion der Turbulenzflecken. a) zeigt den zeitlichen Verlauf des Messignals und b) die dazugehörige Wavelet-Transformation. Die Skalierung der Wavelet-Transformation (c) mit dem Spektrum der laminaren Grenzschicht erleichtert die Detektion der Turbulenzflecken gegenüber Störsignalen wie den Schwankungen in der Außenströmung. In d) sind die berechnete Selektionsfunktion $S F(t)$, der Schwellenwert $S$ und die daraus

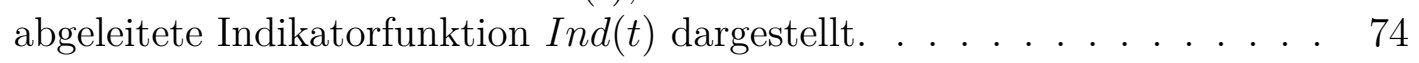

4.13 Beispiel für das gemittelte Wavelet-Spektrum einer laminaren Grenzschicht 75

4.14 Wahrscheinlichkeitsdichteverteilung der Selektionsfunktion für $R e_{1}=$ $24 \cdot 10^{6} \mathrm{~m}^{-1}$ an diversen $x$-Positionen. Aufgrund der Transition findet eine Verlagerung der Wahrscheinlichkeitsdichte zu größeren SF-Werten statt. . 76 
4.15 Wahrscheinlichkeitsdichte von $S F$ für eine laminare Grenzschicht unmittelbar stromauf des Transitionsbeginns. Die Wahrscheinlichkeitsdichte kann durch eine logarithmische Normalverteilung nachgebildet werden. 77

4.16 St-Verteilungen für diverse $R e_{1}$ an der Plattenposition A. Markiert sind die bestimmten $R e_{\mathrm{Tb}_{\mathrm{b}}}$ und $R e_{\mathrm{Te}}$-Punkte sowie die daraus bestimmten Korrelationen $S t_{\mathrm{Tb}}$ und $S t_{\mathrm{Te}}$. Die gestrichelten vertikalen Linien zeigen die mit der Ross-Korrelation (Ross 1972$)$ abgeschätzten $R e_{\mathrm{Te}}$-Positionen für $R e_{1}=8 \cdot 10^{6} \mathrm{~m}^{-1}$ und $R e_{1}=14 \cdot 10^{6} \mathrm{~m}^{-1}$. . . . . . . . . . . . . 78

4.17 Vergleich der bestimmten Intermittenzverteilung anhand unterschiedlicher Verfahren und Strömungsgrößen für die untersuchten $R e_{1}$ an der Plattenposition A . . . . . . . . . . . . . . . . . . . . 80

4.18 Schattenaufnahmen von der Umströmung der ebenen Platte mit montiertem Stoßgenerator. . . . . . . . . . . . . . . . . . . . 83

4.19 Bestimmung des einfallenden Verdichtungsstoßes mittels linearer Regression für 200 Einzelbilder . . . . . . . . . . . . . . . . . . . . . 85

4.20 Beispiel für die St-Verteilung mit SGWW (a) und der spannweitig gemittelte $S t$-Verlauf im Vergleich zum ungestörten Fall (b) bei $R e_{1}=24 \cdot 10^{6} \mathrm{~m}^{-1} 87$

4.21 St-Verteilung mit SGWW bei $R e_{1}=24 \cdot 10^{6} \mathrm{~m}^{-1}$ für diverse $x_{\mathrm{imp}} . \ldots$. . 89

$4.22 S t$-Verteilungen bei der Stoßintensität $p_{3} / p_{1}=2.29$ für diverse $R e_{1}$. . . . 91

4.23 St-Verläufe bei $R e_{1}=19 \cdot 10^{6} \mathrm{~m}^{-1}$ und diversen Stoßintensitäten . . . . . 92

4.24 St-Verläufe bei $R e_{1}=8 \cdot 10^{6} \mathrm{~m}^{-1}$ und $p_{3} / p_{1}=1.76$ für diverse $x_{\text {imp }}$. Die

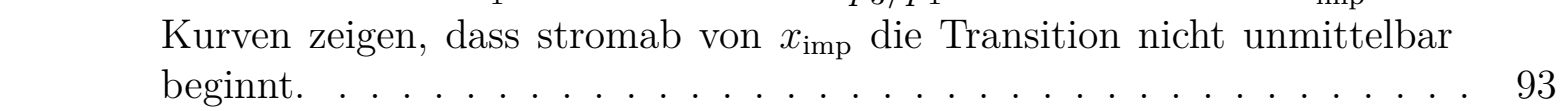

4.25 Die St-Verteilung (a) bei $p_{3} / p_{1}=2.95$ und $R e_{1}=8 \cdot 10^{6} \mathrm{~m}^{-1}$ zeigt spannweitige Variationen stromab der Stroßauftreffposition, die auf GörtlerWirbel zurückgeführt werden. Anhand des spannweitigen St-Verlaufs (b) bei $x=300 \mathrm{~mm} \pm 5 \mathrm{~mm}$ wurde die Wellenlänge der Wirbel bestimmt. . . 94

4.26 Die obere Zeile zeigt den St-Verlauf der untersuchten SGWW mit einer Stoßintensität von $p_{3} / p_{1}=2.29$. In der unteren Zeile sind die Intermittenzverteilungen dargestellt, die aus den Zeischrieben der FLDI-Technik bzw. den PCB-Sensoren berechnet wurden. Markante Stellen der SGWW wurden mittels vertikaler Linien durch beide Diagramme gekennzeichnet. 95

4.27 Fortsetzung von Abb. $4.26 \ldots \ldots \ldots$. . . . . . . . . . . 96

4.28 LDS bei einer SGWW an diversen Plattenpositionen für $R e_{1}=$ $19 \cdot 10^{6} \mathrm{~m}^{-1}, p_{3} / p_{1}=2.29$ und $x_{\mathrm{imp}}=214.1 \mathrm{~mm}$. . . . . . . . . . . . . 98

4.29 Schematische Darstellung der Stantonzahlverteilung mit und ohne SGWW für eine konstante $R e_{1}$ (angepasst aus (Schülein 2014)). Gekennzeichnet sind die charakteristischen Größen des St-Verlaufs. .

4.30 Zur Überprüfung des Skalierungsansatzes von Needham $(1965)$ wurden die experimentellen Daten in die Darstellung von Needham (1965) eingetragen. Da in den eigenen Messungen der Plateaudruck stromab des Ablösestoßes nicht gemessen wurde, wird die Stoßintensität stattdessen über das Druckverhältnis $p_{3} / p_{1}$ berücksichtigt. . . . . . . . . . . . . . . . 104 
4.31 Skalierungsansätze für die Länge der Ablöseblase bei transitionellen SGWW. Zur Entdimensionalisierung der Ablöselänge wird einmal die Lauflänge $x_{\text {ui }}$ (a) und einmal die Verdrängungsdicke $\delta_{1, \text { ui }}(\mathrm{b})$ verwendet. . 105

4.32 Stoßinduzierte maximale Wärmestromdichte im Verhältnis zum Niveau der ungestörten Referenzströmung in Abhängigkeit des Grenzsichtzustandes an der Stoßauftreffposition. . . . . . . . . . . . . . . . . 106

4.33 Skalierte maximale Wärmestromdichte bei transitionellen SGWW für unterschiedliche Stoßintensitäten und $R e_{1}$. . . . . . . . . . . . . . . . . . . 107

4.34 Zusammenhang zwischen $\zeta_{\text {imp }}$, der Distanz bis zum Erreichen des Transitionsendes und die Auswirkung auf die stoßinduzierte Wärmestromdichte. 109

4.35 Vergleich der skalierten maximalen Wärmestromdichte bei transitionellen SGWW zwischen verschiedenen Messkampagnen mit unterschiedlichen Vorderkantenradien der ebenen Platte. . . . . . . . . . . . . . . . . 110

4.36 Durch eine zusätzliche Skalierung der Stoßintensität nach Holden (1977) ergibt sich ein einheitlicher Kurvenverlauf über $\zeta_{\text {imp }}$ für die skalierten maximalen Wärmestromdichten bei transitionellen SGWW. . . . . . . . . 111

4.37 Bei der Verwendung eines theoretischen Referenzwertes in Form der Van Driest II Abschätzung kann der Einfluss der expliziten Strömungssituation auf die stoßinduzierten Wärmestromdichte nicht adäquat nachgebildet werden, wodurch kein einheitlicher Kurvenverlauf entsteht. . . . . . . . . 112

4.38 Die Verwendung von $S t_{\mathrm{Te}}\left(R e_{\mathrm{imp}}\right)$ als Referenzgröße ergibt auch einen einheitlichen Kurvenverlauf, der auch für eine Prognose der stoßinduzierten Wärmelasten bei transitionellen SGWW verwendet werden kann. . . . . 113

A.1 Einbauanleitung der PCB-Sensoren . . . . . . . . . . . . . . . . . . . . 119

A.2 Datenblatt der verwendeten $P C B-132 A 31$ Sensoren . . . . . . . . . . . . 120 



\section{Tabellenverzeichnis}

2.1 Ähnlichkeitslösungen der kompressiblen laminaren Grenzschicht für repräsentative Strömungsbedingungen am RWG . . . . . . . . . . . . . . . 10

3.1 Strömungsparameterbereich des Rohrwindkanals Göttingen . . . . . . . . 26

3.2 Repräsentative Strömungsparameter bei den untersuchten Einheitsreynoldszahlen am RWG . . . . . . . . . . . . . . . . . . 26

$3.3 \quad$ Koordinaten der Stoßgeneratorspitze für die maximal stromauf gelegene Stoßgeneratorposition (Pos. I). . . . . . . . . . . . . . . . . . . 29

3.4 Relevante Stoffgrößen des Plexiglas-Einsatzes bei $T_{\mathrm{w}}=300 \mathrm{~K}$. . . . . . . 32

3.5 Komponenten des verwendeten FLDI Aufbaus . . . . . . . . . . . . . . . 42

$4.1 \quad$ Nicht-viskose Stoßauftreffposition anhand der Schattenaufnahmen für die Stoßgeneratorposition Pos. I bei verschiedenen Anstellwinkeln . . . . . . 84

A.1 Zuordnung zwischen der Einbauposition bei der PCB-Einsatzplatte und dem verwendeten PCB-Sensor. Zu jeder Einbauposition sind die beiden Messpositionen im Koordinatensystem der ebenen Platte angegeben. . . . 120

A.2 Strömungsparameter stromab des einfallenden (G2) und reflektierten (G3) Stoßes. Die Strömungsgrößen $p, \rho, T$ und $u$ sind mit den Anströ-

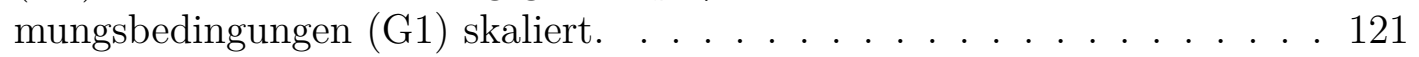

A.3 Übersicht zur Messmatrix bei Pos. A . . . . . . . . . . . . . . . . . . . . . 121

A.4 Übersicht zur Messmatrix bei Pos. B. Der Stoßgenerator wurde um $10 \mathrm{~mm}$

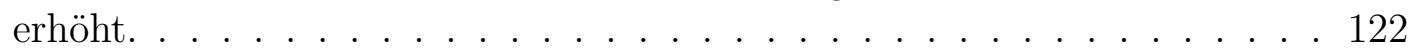

A.5 Übersicht zur Messmatrix der Schattenaufnahmen . . . . . . . . . . . . . 122

A.6 Werte für den Transitionsbeginn bzw. das Transitionsende anhand der St-Messungen für die Plattenposition A. Zusätzlich sind die bestimmten Fitparameter zur Berechnung von $S t_{\mathrm{Tb}}$ und $S t_{\mathrm{Te}}$ aufgelistet. . . . . . . . 123

A.7 Werte für das Transitionsende anhand der St-Verteilung für die Plattenposition B. Da für den stromabgelegenen Messbereich zwei Kurven vorliegen, gibt es zum Transitionsende jeweils zwei Punkte. Zusätzlich sind die bestimmten Fitparameter zur Berechnung von $S t_{\mathrm{Te}}$ aufgelistet. Die Stellen des Transitionsbeginns konnten bei dieser Plattenposition nicht aus den St-Verläufen bestimmt werden, da der Verlauf der Wärmestromdichte durch Störungen von der Lavaldüse beeinflusst ist. . . . . . . . . . . . . . 123 



\section{Einleitung}

Die Umströmung eines Über-/Hyperschallfluggeräts ist nicht nur von Verdichtungsstößen geprägt, die an dessen Oberfläche entstehen, sondern auch von der viskosen Scherschicht, die eine sogenannte Grenzschicht zwischen der Wand und der nicht-viskosen Außenströmung darstellt. Die Interaktion der Verdichtungsstöße mit dieser viskosen Grenzschicht, die im Allgemeinen als Stoß-Grenzschicht-Wechselwirkung (SGWW) bekannt ist, stellt hier die größte Herausforderung bei einer genauen Vorhersage der zu erwarteten thermischen und mechanischen Lasten dar. Abbildung 1.1 verdeutlicht die Vielfalt an SGWW, die an einem Fluggerät im Überschall auftreten können.


Abbildung 1.1: Beispiele von SGWW am Überschallfluggerät (Babinsky \& Harvey 2011)

Ein Verdichtungsstoß verursacht an der Wand einen starken Druckanstieg, wodurch die Grenzschicht aufdickt und gegebenenfalls von der Oberfläche ablöst. Die stoßinduzierte Ablösung kann z. B. die Steuerklappenwirksamkeit stark beeinträchtigen oder zum Leistungseinbruch eines Überschalllufteinlaufs bzw. des gesamten luftatmenden Triebwerks führen. Beim Wiederanlegen der abgelösten Scherschicht entstehen lokale Wärmelasten, 
die um ein Vielfaches größer sind als in der ungestörten Grenzschicht. Bei höheren Machzahlen kann die lokale Aufheizung zur Zerstörung des Fluggeräts führen (Babinsky \& Harvey 2011).

Der Einsatzbereich von Überschallfluggeräten erstreckt sich bis in die Stratosphäre und umfasst einen weiten Einheitsreynoldszahlbereich. In großen Höhen ist die Grenzschicht des Fluggerätes vollständig laminar (Hirschel 2005), aber im Sinkflug steigt die Reynolds-Zahl und schließlich beginnt am Heck der laminar-turbulente Umschlag. Mit wachsender Reynolds-Zahl verschiebt sich der Transitionsbeginn stromauf und durchläuft schließlich den Ort der SGWW, sodass der Grenzschichtzustand bei SGWW mit der Flugsituation variiert. In der Designphase des Fluggerätes müssen daher die thermischen und mechanischen Lasten für sämtliche Fälle berücksichtigt werden. Da die SGWW wesentlich von der viskosen Scherschicht abhängt, entfallen analytische Berechnungen zur Beschreibung der Phänomene bei SGWW. Auch numerische Simulationen mittels RANS haben Probleme die Größe des Ablösegebietes oder die Wärmestromdichte korrekt vorherzusagen.

Seit den 1940ern werden SGWW an generischen Konfigurationen untersucht. Die bisherigen Erkenntnisse sind in mehreren Übersichtsartikeln (Délery \& Marvin 1986, Dolling 2001, Babinsky \& Harvey 2011, Gaitonde 2015) zusammengefasst. Die Datenbasis ermöglichte die Identifizierung der wichtigsten Parameter bei SGWW und die Ausarbeitung von Modellvorstellungen. Es wurden Korrelationsgesetze für wichtige Strömungsgrößen erarbeitet, die unter anderem die Größe der Ablöseblase, die Länge des stromauf beeinflussten Gebietes, die Wanddruckverteilung im Interaktionsbereich und die Wärmestromdichte stromab der Wiederanlegung beschreiben. Die Datenbasis und die Korrelationsgesetze wurden auch zur Validierung und Weiterentwicklung von numerischen Simulationen eingesetzt. Inzwischen genügen die gewonnenen Erkenntnisse bezüglich laminarer SGWW, um mittels numerischer Simulationen eine zufriedenstellende Berechnung der Strömungparameter bei laminaren SGWW durchzuführen (Knight \& Degrez 1998). Bei turbulenten SGWW ist das Verständnis über die zugrundeliegenden Mechanismen noch unzureichend, um diese adäquat in Simulationen nachzubilden (Knight \& Degrez 1998, Gaitonde 2015). Allerdings basieren die erarbeiteten Korrelationen für turbulente SGWW auf einer breit aufgestellten experimentellen Datenbasis, sodass die relevanten Strömungsgrößen zumindest vertretbar abgeschätzt werden können.

Zu transitionellen SGWW liegen im Vergleich zu laminaren und turbulenten SGWW nur wenige experimentelle und numerische Untersuchungen vor. Viele Aspekte bei transitionellen SGWW sind noch unverstanden bzw. unzureichend untersucht, sodass die Vorhersage von Strömungsgrößen großen Ungenauigkeiten unterliegt. Die Kombination aus SGWW und Transition ist besonders anspruchsvoll, weil sie eine gegenseitige Wechselwirkung beider Phänomene beinhaltet. Die SGWW verändert sich mit dem Transitionszustand der Grenzschicht und gleichzeitig beschleunigt die SGWW die natürliche Transition. Zudem hängt die natürliche Transition stark von den Randbedingungen des Windkanalmodells und der Strömungsqualität im Windkanal ab, was sowohl die Reproduzierung der Messdaten als auch den Vergleich zwischen verschiedenen Windkanalanlagen erschwert. Zusätzlich besitzen transitionelle SGWW eine Vielzahl an Einflussparametern, die bei experimentellen Parameterstudien oftmals nicht individuell variierbar 
sind. Wegen dieser Herausforderungen existieren kaum hochwertige Valdierungsdaten zu transitionellen SGWW. Einen aktuellen Überblick über die durchgeführten Untersuchungen zu transitionellen SGWW geben Knight \& Mortazavi (2017).

Eine der ersten Studien zum Einfluss der Transition auf die Länge von stoßinduzierten Ablöseblasen wurde von Needham \& Stollery (1966) durchgeführt. Sie zeigten, dass sich für laminare und turbulente SGWW zwei unterschiedliche Niveaus bei der Größe der Ablöseblase ergeben. Vereinfacht gesehen, stellen die transitionellen SGWW einen Übergang zwischen diesen beiden Niveaus dar. Je weiter die Transition am Interaktionsgebiet fortgeschritten ist, desto kleiner ist die Ablöseblase aufgrund des gestiegenen Impulsaustausches innerhalb der Grenzschicht.

Eine weitere Fragestellung bei transitionellen SGWW betrifft die Auswirkung der SGWW auf die Entwicklung des laminar-turbulenten Umschlags. Pagella et al. (2002) zeigten anhand von direkten numerischen Simulationen und linearen Stabilitätsanalysen, dass Instabilitäten durch die SGWW verstärkt anwachsen. Zusätzlich werden durch die SGWW weitere Störungen in die Grenzschicht eingebracht. Beide Effekte können den laminar-turbulenten Umschlag auslösen oder bei bereits transitionellen SGWW den Prozess signifikant beschleunigen (Hung \& Barnett 1973). Experimentelle Untersuchungen zur Entwicklung der Transition bzw. der Instabilitäten bei SGWW stellen eine Herausforderung dar, weil die Frequenzen der Instabilitäten bei Überschallströmungen oft deutlich über $100 \mathrm{kHz}$ liegen und damit messtechnisch kaum zu erfassen sind. Gleichzeitig sind die Störungsamplituden unter den rauen Messbedingungen in Überschall-Windkanalanlagen nur schwer zu detektieren.

Aufgrund der hohen thermischen Lasten im Überschall gehört die stoßinduzierte Wärmestromdichte zu den kritischsten Parametern bei SGWW. Trotz dieser Bedeutung ist bis heute unklar wie die maximalen Wärmelasten bei transitionellen SGWW abgeschätzt werden können. Eine erste Korrelationsgleichung wurde von Hung \& Barnett (1973) vorgestellt und basiert auf einer Skalierung der maximalen Wärmestromdichte mit einem Referenzwert aus einer laminaren Grenzschicht. Die experimentellen Messungen von Heffner et al. (1993) übertrafen die Korrelation von Hung \& Barnett (1973) allerdings um eine Größenordnung. In einem anderen Skalierungsgesetz von Simeonides et al. (1994) folgen die maximalen Wärmestromdichten bei transitionellen SGWW dem selben Verlauf wie bei turbulenten SGWW. Aktuellere Untersuchungen (Longo 2003, Benay et al. 2006, Schülein 2014, Willems et al. 2015, Currao et al. 2020) vermitteln jedoch einen anderen Eindruck: Die erzeugten Wärmelasten bei transitionellen SGWW übersteigen sowohl die Fälle mit laminaren als auch turbulenten SGWW. Die Unklarheiten bei der Abschätzung der maximalen Wärmestromdichten sind zum Teil der geringen Anzahl an Datensätzen und überschaubaren Parameterstudien geschuldet.

In der Arbeit von Schülein (2014) wurden die Wärmelasten bei transitionellen SGWW zunächst für eine konstante Stoßintensität untersucht und in einer nachfolgenden Messkampagne (Lunte \& Schülein 2019) um zusätzliche Stoßintensitäten erweitert. Beide Studien deuten an, dass transitionelle SGWW nahe des Transitionsbeginns nicht unmittelbar den laminar-turbulenten Umschlag abschließen, was sich auf den Verlauf sowie den Maximalwert der Wärmestromdichte auswirkt. Die aktuelle Arbeit knüpft an den Erkenntnissen von Schülein (2014) und Lunte \& Schülein (2019) an. Durch Anpassungen 
des Windkanalmodells (Abrundung der Modellvorderkante, Verschiebung sowie Erweiterung des Messbereichs) können nun Parameterstudien von SGWW im Bereich des Transitionsbeginns durchgeführt werden.

\subsection{Zielsetzung und Gliederung}

In der aktuellen Arbeit werden systematische Validierungsdaten zu transitionellen SGWW erzeugt und dazu genutzt, die physikalischen Modellvorstellungen auszubauen. Zur Erreichung dieses Ziels wurde am Rohrwindkanal Göttingen (RWG) bei Mach 6 zunächst die transitionelle Grenzschicht entlang einer ebenen Platte untersucht. Dafür wurden von der Strömung Schattenaufnahmen erstellt und die Stantonzahlverteilung auf der Oberfläche mit quantitativer Infrarot-Thermographie (QIRT) vermessen. Zusätzlich wurden im Rahmen der Promotion zwei weitere Messtechniken am RWG eingerichtet. Zum einen wurden die Druckschwankungen an der Modelloberfläche mittels piezoelektrischer Sensoren und zum anderen die Dichteschwankungen innerhalb der Grenzschicht mittels fokussierter Laser-Differentialinterferometrie (FLDI) gemessen. Erst die beiden zusätzlichen Messtechniken ermöglichen die Untersuchung der hochfrequenten Instabilitäten innerhalb der transitionellen Grenzschicht. Die Vermessung der diversen Strömungsparameter ergibt ein umfangreiches Gesamtbild der Grenzschichttransition, das zum einen zur quantitativen Beschreibung der Grenzschicht dient. Zum anderen werden die Daten dazu genutzt, einen skalaren Parameter (Intermittenzwert) zur vereinfachten Kennzeichnung des Grenzschichtzustandes abzuleiten.

In einem zweiten Schritt werden mit den gleichen Messtechniken zweidimensionale SGWW untersucht, die durch einen einfallenden Verdichtungsstoß initiiert werden. Der Verdichtungsstoß wird mittels eines keilförmigen Verdrängungskörpers oberhalb der ebenen Platte erzeugt. Die einfache Modellgeometrie ermöglicht die unabhängige Untersuchung diverser Parameter. Zu diesen Parametern gehört die Stoßauftreffposition und die Stoßintensität. Zusätzlich wurde die Grenzschicht über die Anströmbedingungen variiert. Die zweidimensionalen SGWW auf der ebenen Platte stellen eine idealisierte Messumgebung dar, die eine fokussierte Analyse der SGWW ermöglicht. Da zu den SGWW auch die jeweilige ungestörte Strömungssituation gemessen wurde, können die Veränderungen durch die SGWW identifiziert werden. Diese Maßnahmen ermöglichen die Durchführung einer systematischen Parameterstudie, anhand derer die strömungsphysikalischen Effekte bei SGWW untersucht werden. Auf Basis dieses Datensatzes werden Korrelationen zu charakteristischen Größen erarbeitet.

Die Dissertation ist im Folgenden in vier Kapitel strukturiert. Kapitel 2 behandelt die theoretischen Hintergründe zu den Themengebieten der kompressiblen Grenzschicht und SGWW. Der Windkanal und das verwendete Modell werden zu Beginn von Kapitel 3 vorgestellt. Daran anschließend werden die Versuchsaufbauten der einzelnen Messverfahren sowie die Aufbereitung der Messdaten erläutert. Kapitel 4 zur Diskussion der experimentellen Ergebnisse ist zweigeteilt. Es beginnt mit einer Betrachtung der ungestörten Transition auf der ebenen Platte, die in der Ausarbeitung eines Intermittenzwertes mündet. Erst im zweiten Teil werden die Auswirkung der SGWW untersucht, mit den 
Referenzdaten der ungestörten Plattenumströmung verglichen und Korrelationen bezüglich charakteristischer Größen dargestellt. Die zentralen Erkenntnisse der Dissertation werden in Kapitel 5 zusammengefasst. 



\section{Theorie}

In diesem Kapitel werden die Grundlagen zu SGWW und Grenzschichten in Überschallanströmungen vorgestellt. Dabei erfolgt die Betrachtung der drei Grenzschichtzustände jeweils in einem separaten Abschnitt, wo die charackteristischen Merkmale dargestellt und soweit möglich etablierte Korrelationen zur Berechnung der Strömungsparameter eingeführt werden. Der Abschnitt zu SGWW konzentriert sich auf die Erläuterung der entstehenden Strömungstopologie und betrachtet die Fälle mit und ohne stoßinduzierter Ablösung. Auf eine Vorstellung der Stoßbeziehungen und isentropen Gasrelationen wird in dieser Arbeit verzichtet.

\subsection{Grenzschichten in kompressiblen Strömungen}

Umströmt ein Fluid eine Wand, so erfüllt die Strömung im Allgemeinen die Haftbedingung (Schlichting \& Gersten 2006). Die Haftbedingung fordert, dass die Strömungsgeschwindigkeit tangential zur Wand null ist. Durch diese Randbedingung entsteht ein Übergangsgebiet zwischen der Wand und der endlichen Geschwindigkeit der Anströmung. Dieses Übergangsgebiet wird als Grenzschicht bezeichnet und ist stark durch die Viskosität des Fluids beeinflusst. Die Dicke der Grenzschicht hängt von der Reynoldszahl ab und ist für Luft typischerweise im Millimeterbereich. Nach der Prandtlschen Grenzschichttheorie (Schlichting \& Gersten 2006) kann das Strömungsfeld in eine Außenströmung, in der viskose Effekte vernachlässigt werden können, und in die Grenzschicht unterteilt werden. Abhängig von der Lauflänge an der Modellwand verändert sich die interne Struktur der Grenzschicht. Bei kleinen Lauflängen ist die Grenzschicht laminar und geht dann im Zuge des laminar-turbulenten Umschlag, der auch als Transition bezeichnet wird, in eine turbulente Grenzschicht über. Die folgenden Unterkapitel betrachten jeden Zustand einzeln.

\subsubsection{Laminare Grenzschicht}

Eine Grenzschicht wird als laminar bezeichnet, falls nur ein schwacher Austausch der Fluidteilchen quer zur Strömungsrichtung existiert (Schlichting \& Gersten 2006). Als Folge strömt das Fluid innerhalb der Grenzschicht in Schichten mit unterschiedlicher Geschwindigkeit nebeneinander. Die Form dieses Grenzschichtprofils kann anhand der Grenzschichtgleichungen näherungsweise berechnet werden. Bei den Grenzschichtgleichungen handelt es sich um ein Gleichungssystem, dass durch diverse Vereinfachungen aus den Navier-Stokes-Gleichungen abgeleitet wird. Für zweidimensionale kompressible Grenzschichten ist die Herleitung der Grenzschichtgleichungen beispielsweise in White 
(1991) dargestellt. Für die Aufstellung der Grenzschichtgleichungen wird das Koordinatensystem $(x, y)$ so gewählt, dass es tangential $(x)$ und normal $(y)$ zur Wand zeigt. In den Navier-Stokes-Gleichungen werden die einzelnen Terme auf ihre Größenordnung abgeschätzt und irrelevante Terme aus den Gleichungen entfernt. Daraus ergibt sich, dass der Druck $p$ senkrecht zur Wand konstant ist. Zusätzlich soll das Fluid der Zustandsgleichung idealer Gase folgen. Nach White (1991) ergibt sich schließlich für die zweidimensionale ebene Platte folgendes Gleichungssystem für die Berechnung der Ähnlichkeitslösungen:

$$
\begin{aligned}
\left(\frac{\rho \mu}{\rho_{\infty} \mu_{\infty}} f^{\prime \prime}\right)^{\prime}+f f^{\prime \prime} & =0 \\
\left(\frac{\rho \mu}{\rho_{\infty} \mu_{\infty}} g^{\prime}\right)^{\prime}+\operatorname{Pr} f g^{\prime} & =-\frac{\rho \mu}{\rho_{\infty} \mu_{\infty}} \operatorname{Pr}(\kappa-1) M a_{\infty}^{2} f^{\prime \prime 2}
\end{aligned}
$$

mit den Funktionen

$$
f^{\prime}(\eta)=\frac{u(\eta)}{u_{\infty}}, \quad g(\eta)=\frac{h(\eta)}{h_{\infty}}
$$

und den Ähnlichkeitsvariablen

$$
\xi(x)=\int_{0}^{x} \rho_{\infty} u_{\infty} \mu_{\infty} \mathrm{d} \tilde{x}=\rho_{\infty} u_{\infty} \mu_{\infty} x, \quad \eta(x, y)=\frac{u_{\infty}}{\sqrt{2 \xi(x)}} \int_{0}^{y} \rho \mathrm{d} \tilde{y} .
$$

In den obigen Gleichungen steht $u$ für die Strömungsgeschwindigkeit tangential zur Wand, $M a$ für die Machzahl, $\rho$ für die Dichte, $\mu$ für die dynamische Viskosität, $\operatorname{Pr}$ für die Prandtl-Zahl, $\kappa$ für den Isentropenexponent und $h$ für die spezifische Enthalpie. Der Ableitungsstrich in den Gleichungen bedeutet die Differentiation nach $\eta$. Der Index $\infty$ beschreibt in den Grenzschichtgleichungen streng genommen den Wert des jeweiligen Strömungsparameters am Grenzschichtrand. In dieser Arbeit wird für die ebene Platte angenommen, dass die Strömungsparameter am Grenzschichtrand identisch zur Anströmung sind. Für die Funktionen $f$ und $g$ gelten die folgenden Randbedingungen:

$$
\begin{aligned}
\eta=0: & f=0, \quad f^{\prime}=0, \quad g=g_{\mathrm{w}} \\
\eta \rightarrow \infty: & f^{\prime}=1, \quad g=1 .
\end{aligned}
$$

Das Gleichungssystem 2.1 und 2.2 kann durch numerische Integration gelöst werden, indem die Anfangswerte aus Gleichung (2.5) verwendet werden und die Werte von $f^{\prime \prime}(\eta=0)$ und $g^{\prime}(\eta=0)$ systematisch iteriert werden bis die Rangbindungen 2.6 für ein ausreichend großes $\eta$ erfüllt sind.

Für die numerische Integration wurde ein explizites Euler-Verfahren mit einer Schrittweite $\Delta \eta=1 \cdot 10^{-5}$ verwendet und bis zu $\eta=20$ integriert. Die Berechnungen wurden für Luft mit den Stoffwerten $\operatorname{Pr}=0.72, \kappa=1.4$ und der spezifische Gaskonstante $R=287 \mathrm{~J} /(\mathrm{kgK})$ durchgeführt (Schlichting \& Gersten 2006). Für die Abhängigkeit der Viskosität von der Temperatur wird in dieser Arbeit das Sutherland-Gesetz

$$
\mu(T)=\mu_{\text {ref }}\left(\frac{T}{T_{\text {ref }}}\right)^{3 / 2} \frac{T_{\text {ref }}+S}{T+S}
$$


verwendet mit den Referenzgrößen für Luft $\mu_{\text {ref }}=1.716 \cdot 10^{-5} \mathrm{~kg} /(\mathrm{sm}), T_{\text {ref }}=273 \mathrm{~K}$ und $S=111 \mathrm{~K}$ (White 1991). Zusätzlich werden konstante spezifische Wärmekapazitäten angenommen, sodass $g=T / T_{\infty}\left(\right.$ Schlichting \& Gersten 2006) gilt. Die Werte $M a_{\infty}=5.98$, $T_{\infty}=66.6 \mathrm{~K}$ und die Wandtemperatur $T_{\mathrm{w}}=300 \mathrm{~K}$ wurden passend zu den Strömungsbedingungen im Windkanal gewählt (vgl. Abschnitt 3.1). Somit können die Ergebnisse der numerischen Berechnung später als Referenzfall für die laminare Grenzschicht zu den experimentellen Daten verwendet werden.

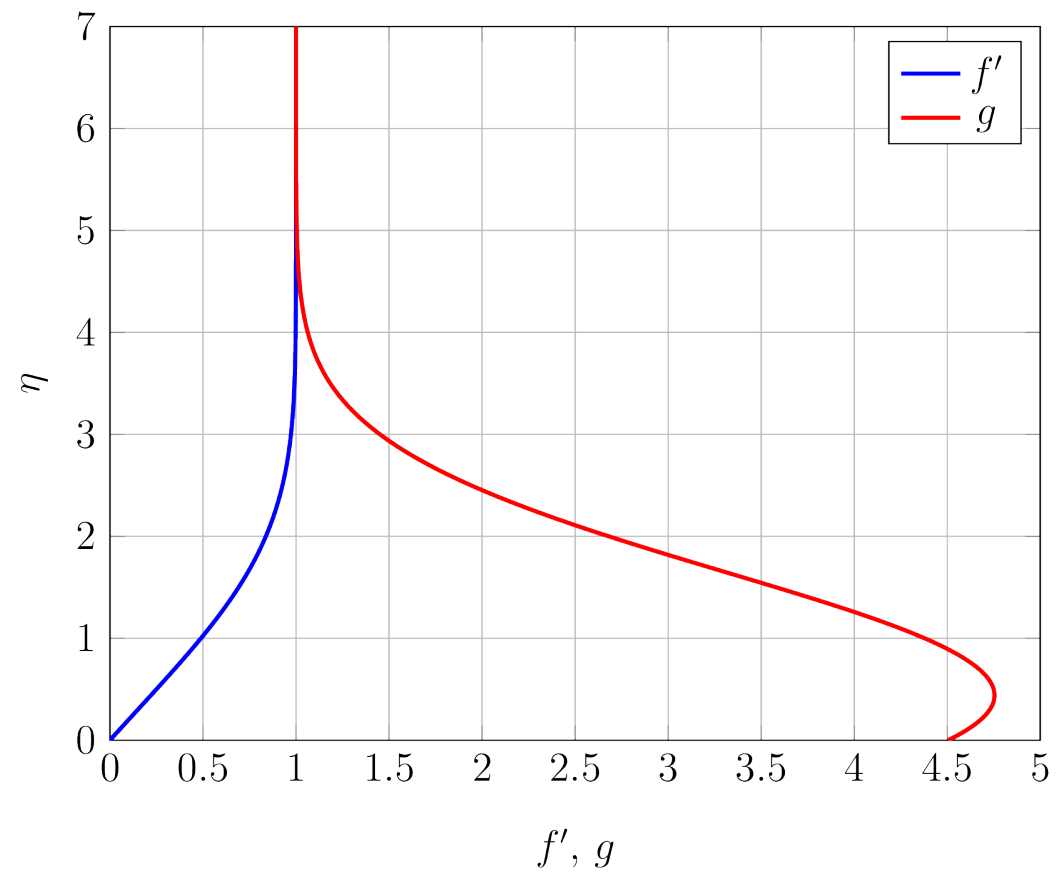

Abbildung 2.1: Grenzschichtprofile der Ähnlichkeitslösungen einer kompressiblen laminaren Grenzschicht für repräsentative Strömungsbedingungen am RWG

Die berechneten Werte für $f^{\prime \prime}(0)$ und $g^{\prime}(0)$ sind in Tabelle 2.1 notiert. In Abbildung 2.1 ist das Grenzschichtprofil von $f^{\prime}$ und $g$ dargestellt. Das Geschwindigkeitsprofil $\left(f^{\prime}\right)$ wächst mit zunehmendem Abstand von der Wand bis es die Geschwindigkeit der Außenströmung erreicht. Im Vergleich dazu erreicht die statische Temperatur $(g)$ den Wert in der Außenströmung $(g=1)$ erst im größeren Abstand zur Wand. Dieser Effekt entsteht, da die Prandtl-Zahl des Fluids kleiner als 1 ist. Die Prandtl-Zahl beschreibt das Verhältnis zwischen kinematischer Viskosität und der Temperaturleitfähigkeit. Für $\operatorname{Pr}<1$ dominiert die Temperaturleitfähigkeit, wodurch Temperaturunterschiede sich über einen größeren Bereich erstrecken. Bei dem Profil von $g$ bildet sich in Wandnähe ein lokales Maximum aus, da die Wandtemperatur $\left(T_{\mathrm{w}}=300 \mathrm{~K}\right)$ kleiner ist als die adiabate Wandtemperatur $T_{\text {aw }}$ und somit das Fluid Wärme in die Wand abgibt. Bei der adiabatischen Wandtemperatur handelt es sich um die Gleichgewichtstemperatur, die das Fluid an der Wand annimmt, falls kein Wärmeaustausch (adiabatisch) mit der Wand stattfindet. Aufgrund von Dissipation innerhalb der Grenzschicht ist $T_{\text {aw }}$ kleiner als die Totaltemperatur $T_{0}$. Der Unterschied zwischen diesen beiden Temperaturen wird 
mit dem Rückgewinnfaktor

$$
r=\frac{T_{\mathrm{aw}}-T_{\infty}}{T_{0}-T_{\infty}}
$$

beschrieben, sodass $T_{\text {aw }}$ mittels

$$
T_{\mathrm{aw}}=T_{\infty}\left(1+r \frac{\kappa-1}{2} M a^{2}\right)
$$

berechnet werden kann (White 1991). Der Rückgewinnfaktor einer laminaren Grenzschicht kann mit Hilfe der Grenzschichtgleichungen bestimmt werden (vorgeführt in White (1991)) und wird für Luft üblicherweise als $r_{\text {lami }}=\sqrt{P r}$ angenommen. Die adiabate Wandtemperatur hat für die Skalierung von Kennzahlen mit Bezug zu Wärmeübergängen eine große Bedeutung, da die Wärmestromdichte proportional zur Temperaturdifferenz $\Delta T=T_{\mathrm{w}}-T_{\mathrm{aw}}$ ist.

Die berechneten Grenzschichtprofile können auch genutzt werden, um die Verteilung der Stanton-Zahl St, des Reibungsbeiwerts $c_{f}$, der Grenzschichtdicke $\delta_{99}$, der Verdrängungsdicke $\delta_{1}$ und der Impulsverlustdicke $\delta_{2}$ auf einer ebenen Platte zu berechnen:

$$
\begin{aligned}
S t & =\frac{g^{\prime}(0) T_{\infty}}{\sqrt{2} \operatorname{Pr}\left(T_{\mathrm{aw}}-T_{\mathrm{w}}\right)} \frac{\rho_{\mathrm{w}} \mu_{\mathrm{w}}}{\rho_{\infty} \mu_{\infty}} \frac{1}{\sqrt{R e_{x}}}=\frac{C_{S t}}{\sqrt{R e_{x}}} \\
c_{f} & =\sqrt{2} f^{\prime \prime}(0) \frac{\rho_{\mathrm{w}} \mu_{\mathrm{w}}}{\rho_{\infty} \mu_{\infty}} \frac{1}{\sqrt{R e_{x}}}=\frac{C_{c_{f}}}{\sqrt{R e_{x}}} \\
\delta_{99} & =\frac{\sqrt{2} x}{\sqrt{R e_{x}}} \int_{0}^{\eta_{99}} g \mathrm{~d} \eta=C_{\delta_{99}} \frac{x}{\sqrt{R e_{x}}} \\
\delta_{1} & =\frac{\sqrt{2} x}{\sqrt{R e_{x}}} \int_{0}^{\infty}\left(g-f^{\prime}\right) \mathrm{d} \eta=C_{\delta_{1}} \frac{x}{\sqrt{R e_{x}}} \\
\delta_{2} & =\frac{\sqrt{2 x}}{\sqrt{R e_{x}}} \int_{0}^{\infty} f^{\prime}\left(1-f^{\prime}\right) \mathrm{d} \eta=C_{\delta_{2}} \frac{x}{\sqrt{R e_{x}}}
\end{aligned}
$$

Die Herleitung dieser Gleichungen ist ausgehend von ihrer jeweiligen Definition im Anhang A.1 vorgeführt. Da sich die Grenzschichtgleichungen auf ein Gleichungssystem gewöhnlicher Differentialgleichungen (Gl. (2.1) und (2.2) reduzieren lassen, ergeben sich für die Strömungsparameter $\left(S t, c_{f}, \delta_{99}, \delta_{1}\right.$ und $\left.\delta_{2}\right)$ analytische Funktionen bestehend aus einer Konstanten, die mit einem Skalierungsfaktor multipliziert wird. Die Ergebnisse für die einzelnen Konstanten sind in Tabelle 2.1 aufgelistet.

\begin{tabular}{ccccccc}
\hline$f^{\prime \prime}(0)$ & $g^{\prime}(0)$ & $C_{S t}$ & $C_{c_{f}}$ & $C_{\delta_{99}}$ & $C_{\delta_{1}}$ & $C_{\delta_{2}}$ \\
\hline 0.49690 & 1.12602 & 0.3955 & 0.6445 & 15.20 & 12.14 & 0.6445 \\
\hline
\end{tabular}

Tabelle 2.1: Ähnlichkeitslösungen der kompressiblen laminaren Grenzschicht für repräsentative Strömungsbedingungen am RWG 


\subsubsection{Turbulente Grenzschicht}

Im Gegensatz zu einer laminaren Strömung wird eine Strömung als turbulent bezeichnet, falls ein signifikanter Austausch an Impuls und Temperatur zwischen den Fluidteilchen quer zur Strömungsrichtung vorliegt (Schlichting \& Gersten 2006). Als Konsequenz fluktuieren die Strömungsgeschwindigkeiten und somit auch sämtliche andere Strömungsgrößen im zeitlichen Verlauf. Diese Schwankungen, die auch als Störungen bezeichnet werden, müssen bei Berechnung von turbulenten Strömungen berücksichtigt werden.

Für die Aufstellung von Grenzschichtgleichungen bedeuten die Schwankungen, dass es keinen stationären Fall gibt. Da eine zeitlich aufgelöste Berechnung der Strömungsgrößen oftmals nicht benötigt wird, wird eine zeitliche Mittelung durchgeführt. In kompressiblen Strömungen wird hierfür die Favre-Mittelung eingesetzt (Schlichting \& Gersten 2006). Als Folge der Mittelung entstehen in den Gleichungen zusätzliche Terme, die sich aus den Schwankungsgrößen zusammensetzen. Da in den gemittelten Gleichungen sowohl die gemittelten Strömungsparameter als auch deren Schwankungen enthalten sind, ist das Gleichungssystem unterbestimmt. Um dieses Gleichungssystem dennoch lösen zu können, sind empirische Korrelationen zur Beschreibung der Schwankungsgrößen notwendig. In numerischen Simulationen werden hierfür speziell erarbeitete Turbulenzmodelle verwendet. Für spezielle Strömungssituationen existieren allerdings auch semi-analytische Lösungen. Im Bereich der Überschallströmungen wird die Lösung von Van Driest (1956) für die Umströmung einer ebenen Platte am häufigsten verwendet (White 1991). Die Lösung wird auch als Van Driest II bezeichnet und liefert nach Cebeci \& Bradshaw (1984) folgende Gleichung für die Berechnung des Reibungsbeiwertes:

$$
\begin{aligned}
& \frac{0.242}{A \sqrt{c_{f}\left(T_{\mathrm{w}} / T_{\infty}\right)}}(\arcsin \alpha+\arcsin \beta)=0.41+\log _{10}\left(R e_{x} c_{f}\right)-\omega \log _{10}\left(T_{\mathrm{w}} / T_{\infty}\right), \\
& \alpha=\frac{2 A^{2}-B}{\sqrt{B^{2}+4 A^{2}}}, \quad \beta=\frac{B}{\sqrt{B^{2}+4 A^{2}}}, \\
& A=\sqrt{\frac{r M a_{\infty}^{2}(\kappa-1) / 2}{T_{\mathrm{w}} / T_{\infty}}}, \quad B=\frac{1+r M a_{\infty}^{2}(\kappa-1) / 2}{T_{\mathrm{w}} / T_{\infty}}-1
\end{aligned}
$$

mit $r=0.89, \omega=0.76$ und $\operatorname{Pr}=0.72$. Zusammen mit der modifizierten Reynoldsanalogie (Van Driest 1956)

$$
S t=\operatorname{Pr}^{-2 / 3} \frac{c_{f}}{2}
$$

kann die dimensionslose Wärmestromdichte entlang einer ebenen Platte bei einer vollturbulenten Grenzschicht abgeschätzt werden.

Der verstärkte Austausch zwischen den Teilchen gegenüber einer laminaren Grenzschicht führt zu einem erhöhtem Impuls im wandnahem Grenzschichtbereich und zu einem völligerem Geschwindigkeitsprofil. Dies macht die turbulente Grenzschicht widerstandsfähiger gegenüber Druckanstiegen, weswegen in turbulenten Grenzschichten gemeinhin kleinere Ablösegebiete entstehen. 
Die Ursache für die signifikanten Schwankungen innerhalb einer turbulenten Strömung liegen in der Nichtlinearität der Navier-Stokes Gleichung. Die Auswirkungen der Nichtlinearität auf die zeitliche Entwicklung von Störungen wird vereinfacht in Bailly \& Comte-Bellot (2015) aufgezeigt. In dieser Vereinfachung wird nur die substantielle Ableitung der Navier-Stokes-Gleichung einer zweidimensionalen Strömung betrachtet. Druck- und Reibungskräfte werden für die Erklärung auf null gesetzt, sodass sich die Impulsgleichungen $\mathrm{zu}$

$$
\begin{aligned}
& \frac{\partial u}{\partial t}+u \frac{\partial u}{\partial x}+v \frac{\partial u}{\partial y}=0 \\
& \frac{\partial v}{\partial t}+u \frac{\partial v}{\partial x}+v \frac{\partial v}{\partial y}=0
\end{aligned}
$$

vereinfachen. Die Störungen der Strömungsparameter können mathematisch durch Schwingungen beschrieben werden. In diesem Fall wird für die Schwankungen der Geschwindigkeitskomponenten $u$ und $v$ in den Raumrichtungen $x$ und $y$ angenommen, dass

$$
\begin{aligned}
& u(x, y, t=0)=A \cos \left(k_{x} x\right) \cos \left(k_{y} y\right) \\
& v(x, y, t=0)=A^{\prime} \cos \left(k_{x}^{\prime} x\right) \cos \left(k_{y}^{\prime} y\right)
\end{aligned}
$$

gilt. Hierbei stehen $k_{x}, k_{x}^{\prime}, k_{y}$ und $k_{y}^{\prime}$ für die Wellenzahlen der Schwingungen. Mittels einer Taylor-Näherung kann die Strömungsgeschwindigkeit nach der Zeit $\Delta t$ bestimmt werden:

$$
\begin{aligned}
u(x, y, \Delta t)= & u(x, y, 0)+\left.\Delta t \cdot \frac{\partial u}{\partial t}\right|_{t=0}+\mathcal{O}\left(\Delta t^{2}\right) \\
= & u(x, y, 0)+\left.\Delta t \cdot\left[-u \frac{\partial u}{\partial x}-v \frac{\partial u}{\partial y}\right]\right|_{t=0}+\mathcal{O}\left(\Delta t^{2}\right) \\
= & u(x, y, 0)+\Delta t \cdot\left[A^{2} k_{x} \cos \left(k_{x} x\right) \sin \left(k_{x} x\right) \cos ^{2}\left(k_{y} y\right)\right. \\
& \left.+A^{\prime} A k_{y} \cos \left(k_{x}^{\prime} x\right) \cos \left(k_{y}^{\prime} y\right) \cos \left(k_{x} x\right) \sin \left(k_{y} y\right)\right]+\mathcal{O}\left(\Delta t^{2}\right) \\
= & u(x, y, 0)+\Delta t \cdot\left[\frac{A^{2} k_{x}}{4} \sin \left(2 k_{x} x\right)\left(\cos \left(2 k_{y} y\right)+1\right)\right. \\
& +\frac{A^{\prime} A k_{y}}{4}\left\{\cos \left(\left(k_{x}+k_{x}^{\prime}\right) x\right)+\cos \left(\left(k_{x}-k_{x}^{\prime}\right) x\right)\right\} \\
& \left.\cdot\left\{\sin \left(\left(k_{y}+k_{y}^{\prime}\right) y\right)+\sin \left(\left(k_{y}-k_{y}^{\prime}\right) y\right)\right\}\right]+\mathcal{O}\left(\Delta t^{2}\right)
\end{aligned}
$$

Wichtig an dieser Rechnung ist zu erkennen, dass eine initiale Störung durch die Nichtlinearität der Navier-Stokes Gleichung über die Zeit sowohl höhere Schwingungsmoden $\left(\cos \left(2 k_{y} y\right)\right.$ und $\left.\sin \left(2 k_{x} x\right)\right)$ als auch neue Schwingungen erzeugt. Die neuen Schwingungen besitzen sowohl kleinere (bspw. $\left.\sin \left(\left(k_{y}-k_{y}^{\prime}\right) y\right)\right)$ als auch größere $\left(\right.$ bspw. $\left.\cos \left(\left(k_{x}+k_{x}^{\prime}\right) x\right)\right)$ 
Wellenzahlen als die Ausgangsschwingung. Da diese Schwingungserzeugung für alle Frequenzen gilt und in der Strömung permanent stattfindet, entsteht ein sehr breitbandiges Frequenzspektrum. Dieses Spektrum ist für kleine Wellenzahlen durch die geometrischen Abmessungen des Modells begrenzt und für große Wellenzahlen durch molekulare Dissipation. In dem Bereich zwischen diesen Extremen kann das Energiespektrum über das Kolmogorov-5/3-Gesetz $E(k) \sim k^{-5 / 3}$ beschrieben werden (Bailly \& Comte-Bellot 2015).

Diese mathematische Veranschaulichung bezüglich des Frequenzspektrums gilt genauso für laminare Grenzschichten, obwohl die laminare Grenzschicht dadurch gekennzeichnet ist, dass interne Störungen vernachlässigbar sind. Dieser Widerspruch löst sich auf, wenn bedacht wird, dass wesentliche Bestandteile der Navier-Stokes-Gleichung für die Berechnung auf null gesetzt wurden. Die mathematische Veranschaulichung zeigt nur, dass durch die Nichtlinearität zusätzliche Wellenzahlen erzeugt werden können. Es liefert allerdings keine Aussage darüber, ob und wie diese Störungen wachsen. So wurde der schwingungsdämpfende Effekt der Viskosität (Oertel 2017) vernachlässigt, der in einer laminaren Grenzschicht das Wachstum der Schwingungen unterbindet. Mehr Informationen über die Entstehung und Entwicklung von Störungen innerhalb der Grenzschicht werden im nächsten Kapitel zur Transition erläutert.

\subsubsection{Transition}

Der Wechsel von einer laminaren zu einer turbulenten Grenzschicht wird als Transition bezeichnet. Obgleich in vielen Arbeiten von einem Transitionspunkt gesprochen wird, erstreckt sich der Transitionsprozess über eine endliche Distanz in Strömungsrichtung. Bei der Transition handelt es sich um ein Stabilitätsproblem (Oertel 2017), bei dem kleine Störungen innerhalb der Grenzschicht nicht mehr gedämpft werden können. Als Folge beginnen die Störungen zu wachsen und leiten den Übergang zur turbulenten Grenzschicht ein. Die Vorgänge während der Transition sind nicht vollständig verstanden, sodass die Transition auch heutzutage ein aktives Forschungsthema der Strömungsmechanik ist. Numerische und experimentelle Untersuchungen zeigten, dass unterschiedliche physikalische Mechanismen zur Transition führen können. Morkovin (1969) begann die verschiedenen Prozesse in einem Flussdiagramm zu visualisieren. Eine moderne Variante des Flussdiagramms ist in Abbildung 2.2 dargestellt.

Im Flussdiagramm beginnt der Transitionsprozess damit, dass externe Störungen auf die laminare Grenzschicht wirken. Die externen Störungen können verschiedene Ursachen haben und wirken sich mannigfaltig auf die Grenzschicht aus. Einige Ursachen sind in Abbildung 2.3 schematisch für eine Strömung im Überschall dargestellt. Mögliche externe Störungen sind die Turbulenz der Anströmung, Oberflächenrauhigkeiten und akustische Wellen. Neben der Art der externen Störung beeinflusst auch deren jeweilige Stärke den Transitionsprozess.

Innerhalb der laminaren Grenzschicht werden durch einen als Rezeptivität bezeichneten Prozess externe Störungen in interne Schwingungen umgewandelt. Die Rezeptivität der Grenzschicht bestimmt die anfängliche Amplitude, Frequenz und Phase der angeregten Schwingungen (Zhong \& Wang 2012). Damit hat die Rezeptivität einen großen Einfluss auf den weiteren Verlauf der Transition (vgl. Abb. 2.2). Die verschiedenen Ver- 


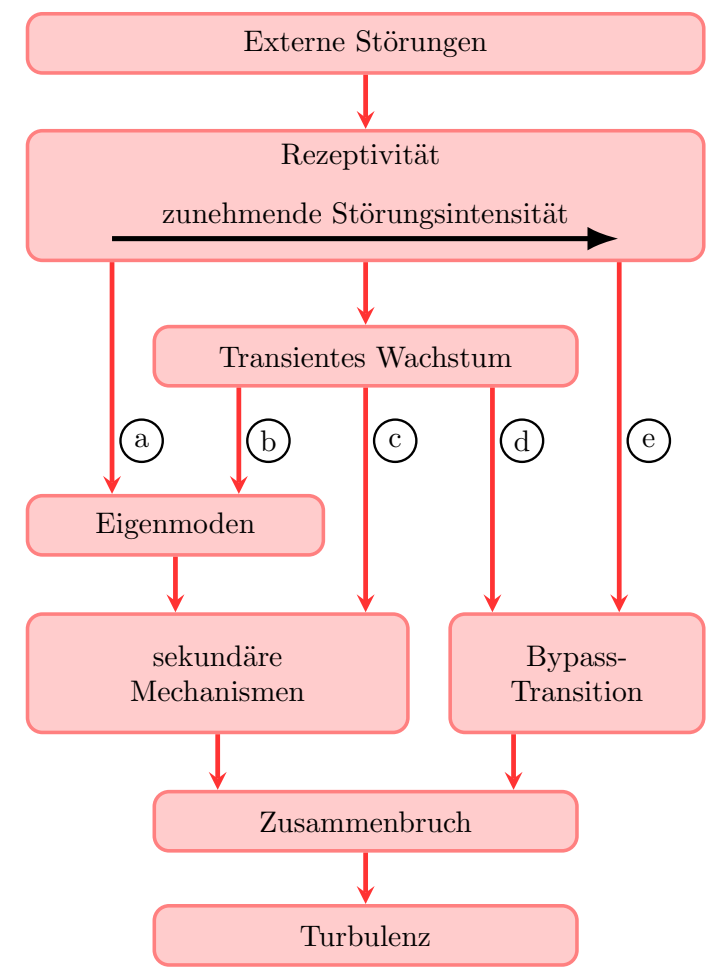

Abbildung 2.2: Flussdiagramm bezüglich der verschiedenen Pfade zur Transition (Abbildung angepasst aus (Fedorov 2011))

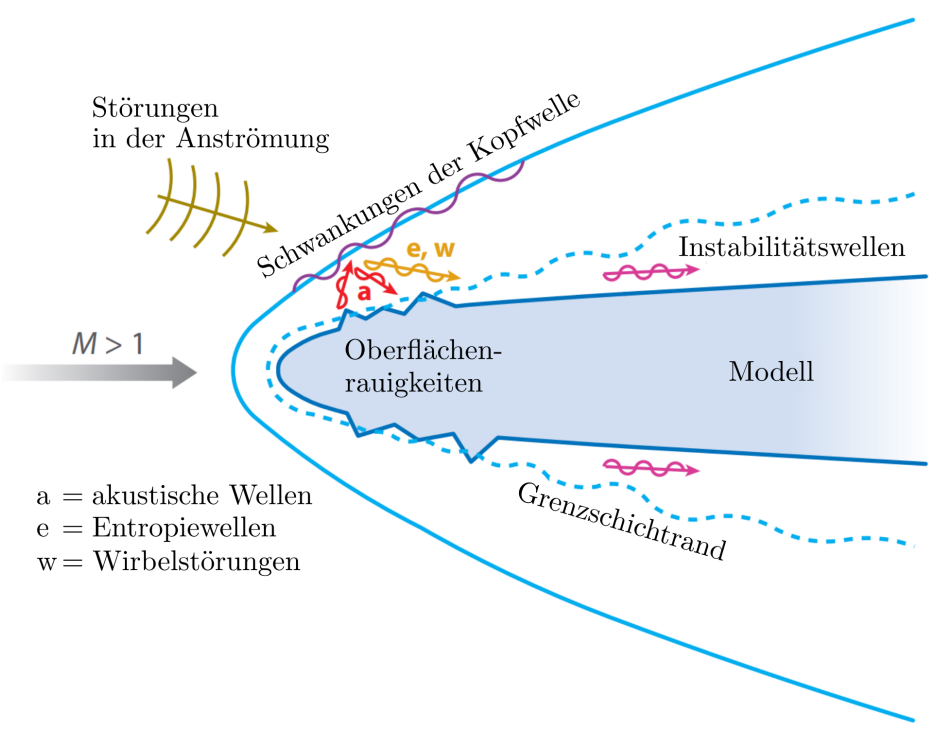

Abbildung 2.3: Skizze von möglichen externen Störungen, die auf eine laminare Grenzschicht im Überschall wirken (übersetzt aus (Zhong \& Wang 2012)) 
läufe sollen im folgenden näher diskutiert werden.

Natürliche Transition Falls die externen Störungen klein sind, werden innerhalb der Grenzschicht durch die Rezeptivität die instabilen Eigenmoden angefacht. Diese Eigenmoden wachsen entlang der Strömungsrichtung und führen sowohl zu dreidimensionalen als auch nichtlinearen Interaktionen, wenn ihre Amplitude ausreichend ist. Die zusätzlichen Interaktionen beschleunigen das weitere Wachstum der Störungen, bis diese Strukturen zusammenbrechen und die turbulente Grenzschicht beginnt (Saric et al. 2002). Dieser Prozess ist in Pfad a dargestellt und wird als natürliche Transition bezeichnet.

Da die Amplitude der angeregten Eigenmoden bei ihrer Entstehung klein ist, kann die lineare Stabilitätstheorie (LST) (Reed et al. 1996) verwendet werden, um das Wachstum der Schwingungen zu berechnen. Bei der LST werden die Navier-Stokes-Gleichungen in eine stationäre Grundströmung und einen instationären Anteil aufgeteilt. Das Gleichungssystem wird anschließend vereinfacht und unter der Voraussetzung kleiner Störungen linearisiert. Unter Vorgabe einer stationären Grundströmung wird analysiert, wie das Gleichungssystem auf eine Störung reagiert. Die LST kann sowohl dazu verwendet werden die instabilen Eigenmoden des Systems zu bestimmen als auch vorherzusagen, wie sich die Moden innerhalb der Grenzschicht entwickeln.

Mack (1984) wendete die LST auf eine kompressible Grenzschicht an einer ebenen Platte an und zeigte, dass abhängig von der Machzahl unterschiedliche Störungen den Transitionsprozess dominieren. Abbildung 2.4 zeigt die zeitlichen Verstärkungsraten zweier Moden für die instabilste Ausbreitungsrichtung $\psi$ der Welle.

Im unteren Machzahlbereich ist die dominante Störung als erste Mode bzw. TollmienSchlichting (TS) Mode (Stetson \& Kimmel 1992) bekannt. Diese Art der Instabilität ist in der Literatur intensiv untersucht worden, da die natürliche Transition im Unterschall meistens durch die TS-Mode hervorgerufen wird. Im Unterschall bilden sich durch die TS-Instabilitäten zweidimensionale Wellen aus, die anwachsen und zu dreidimensionalen $\Lambda$-Wirbeln führen. Diese Wirbel zerfallen schließlich und erzeugen Turbulenzflecken. Hierbei handelt es sich um räumlich begrenzte Gebiete, in denen die Strömung bereits turbulenten Charakter besitzt, während die umgebene Strömung noch laminar ist. Stromab erhöht sich der prozentuale Anteil der turbulenten Strömung aufgrund der zunehmenden Anzahl und Größe der Turbulenzflecken bis eine vollständig ausgebildete turbulente Grenzschicht vorliegt (Oertel 2017).

Für die untersuchte Anströmmachzahl von 6 zeigt Abbildung 2.4, dass die zweite Mode - auch zweite Mack-Mode genannt - für den Transitionsprozess relevant ist. Hierbei handelt es sich um akustische Moden (Mack 1990), die sich bevorzugt in Strömungsrichtung ausbreiten $\left(\psi=0^{\circ}\right)$ und innerhalb der Grenzschicht eingeschlossen sind (Morkovin 1987, Mack 1990). Durch eine thermoakustische Interpretation der zweiten Mack-Moden konnte Kuehl (2018) zeigen, dass innerhalb der Grenzschicht ein Minimum in der akustische Feldimpedanz existiert. Die akustische Feldimpedanz beschreibt den Widerstand den ein Medium der Ausbreitung einer akustischen Welle entgegensetzt. Die Existenz eines Minimums bezüglich der akustische Feldimpedanz behindert eine Emission der akustischen Wellen aus der Grenzschicht. Somit bleibt die Energie der akustischen Wellen inner- 


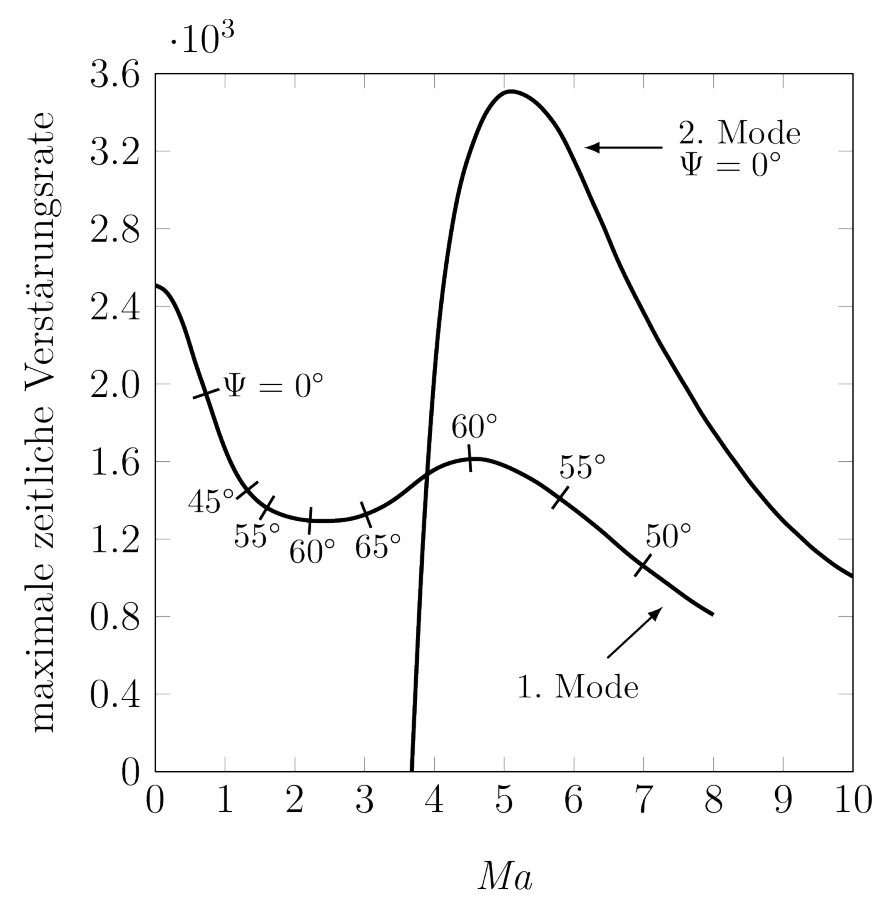

Abbildung 2.4: Einfluss der Anströmmachzahl auf die zeitlichen Verstärkungsraten der ersten und zweiten Störungsmode. Angegeben ist die Verstärkungsrate für den instabilsten Ausbreitungswinkel der Welle (nachgezeichnet aus (Mack 1984))

halb der Grenzschicht. Thermoakustische Reynoldsspannungen in der Grundströmung liefern zusätzliche Energie (Kuehl 2018) für das Anwachsen der zweiten Mack-Moden, sodass die zweiten Mack-Moden zur dominanten Instabilität werden. Der weitere Transitionsprozess verläuft nach dem bereits beschriebenen qualitativen Schema ab. Erreichen die Instabilitäten eine gewisse Intensität führt dies zu sekundären Mechanismen. Durch nichtlineare Effekte können sowohl weitere Moden als auch dreidimensionale Strukturen angeregt werden. Schlussendlich kommt es zum Zusammenbruch und zur Entstehung von Turbulenzflecken.

Bypass-Transition Bislang wurde nur die Situation mit einer turbulenzarmen Anströmung behandelt. Interagieren deutlich stärkere Störungen mit der Grenzschicht, so verläuft die Transition entlang anderer Pfade (vgl. Abb. 2.2). Mit zunehmender Störungsintensität werden die Abläufe der natürlichen Transition teilweise oder ganz übersprungen. Da bei starken Störungen wesentliche Abläufe der natürlichen Transition umgangen werden, bezeichnet man diesen Pfad als Bypass-Transition (Pfad e in Abb. 2.2). Neben den beiden Extremfällen sind noch drei weitere Mechanismen bekannt, die zu einer beschleunigten Transition führen. Diese werden unter dem Begriff transientes Wachstum zusammengefasst (Pfade b, c und d). Hierbei handelt es sich um eigentlich stabile Moden innerhalb der Grenzschicht. Aber durch Überlagerung der Moden können die resultierenden Amplituden vorübergehend (transient) wachsen, bevor die Schwingungen 
wieder gedämpft werden. Je nachdem welches Niveau die Amplituden erreichen, wird der Transitionsprozess unterschiedlich stark beeinflusst. Diese Beeinflussung reicht von einem beschleunigten Ablauf der natürlichen Transition bis zur Bypass-Transition.

Görtler-Wirbel Neben den bisher vorgestellten Transitionsmechanismen, soll an dieser Stelle noch auf eine weitere Instabilität hingewiesen werden, die an konkav gekrümmten Wänden existiert. Ist der Krümmungsradius im Verhältnis zur Grenzschichtdicke ausreichend klein, führt die Trägheit des Fluids zu Instabilitäten innerhalb der laminaren Grenzschicht. Diese Instabilitäten führen zu gegenläufig rotierenden Wirbelpaaren, die sich in Strömungsrichtung erstrecken und allgemein als Görtler-Wirbel bezeichnet werden (Schlichting \& Gersten 2006). Eine Skizze der Wirbelstruktur ist in Abbildung 2.5 dargestellt.

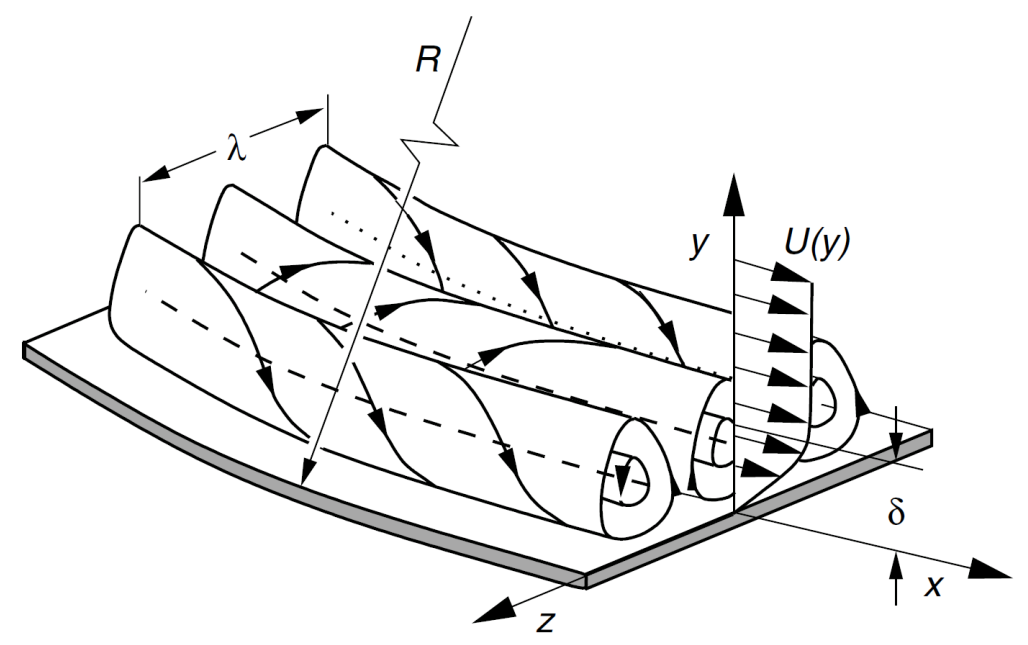

Abbildung 2.5: Görtler-Wirbel innerhalb der Grenzschicht entlang einer konkaven Wand (Schlichting \& Gersten 2006)

Experimentelle Untersuchung der Transition Im Experiment wird die Transition durch viele Faktoren beeinflusst: Machzahl, Wandtemperatur, Einheitsreynoldszahl, Oberflächenrauhigkeiten, Querströmungen, Wandkrümmungen, Vorderkantenradius, Grenzschicht der Überschalldüse etc. (Reshotko 1976). Aufgrund der vielen Einflussfaktoren und der unverstandenen strömungsmechanischen Vorgänge sind experimentelle Messungen bezüglich Transition herausfordernd und schwierig zu vergleichen.

Um die strömungsmechanischen Vorgänge während der Transition besser zu verstehen, werden daher Stabilitätsuntersuchungen durchgeführt. Hierbei werden die angeregten Schwingungen innerhalb der Grenzschicht gemessen, mit dem Ziel theoretische Konzepte zu validieren, die Modellbildung hinter der Transition zu erweitern und die Vorhersage des Instabilitätswachstums innerhalb der laminaren Grenzschicht zu verbessern. Hitzdraht-Messungen bei Mach 8 von Stetson \& Kimmel (1992) zeigten beispielsweise, dass bei den Eigenmoden die größten Schwankungen des Massenstroms bei circa $90 \%$ der 
Grenzschichtdicke $\delta$ vorliegen. Hierbei basieren die Fluktuationen im Massenstrom vor allem auf Dichteschwankungen und nicht auf den Geschwindigkeitsschwankungen. Demetriades (1977) und Stetson et al. (1989) zeigten, dass für die Wellenlänge der zweiten Mack-Mode näherungsweise gilt $\lambda_{\text {Mack }} \approx 2 \delta$, sodass die Frequenz der zweiten Mack-Mode über $f_{\text {Mack }}=u_{\infty} /(2 \delta)$ abgeschätzt werden kann.

Neben den Stabilitätsmessungen werden auch die Auswirkungen des laminarturbulenten Umschlags auf makroskopische Eigenschaften der Strömung wie der Wandschubspannung oder der Wärmestromdichte untersucht. Hierfür wird der Zustand bzw. der Fortschritt der Transition über einen skalaren Intermittenzwert $\gamma$ (Narasimha 1985) quantifiziert. Üblicherweise ist $\gamma$ über den prozentualen Anteil an turbulenter Strömung definiert (Schneider 1995) und somit eng mit den Turbulenzflecken verknüpft. Aus den experimentellen Daten werden Korrelationen zwischen dem Intermittenzwert und anderen Strömungsparametern wie die Länge der Ablöseblase oder der Wärmestromdichte abgeleitet.

\subsection{Stoß-Grenzschicht-Wechselwirkung}

Abbildung 1.1 zeigt die Strömungstopologie an einem Überschallflugzeug, in der eine Vielzahl an unterschiedlichen 2D und 3D SGWW vorliegen. Wegen der modularen Zusammensetzung können durch ein besseres Verständnis der fundamentalen SGWW auch die Eigenschaften des Gesamtflugzeugs optimiert werden. Délery \& Marvin (1986) unterscheiden vier grundlegende Konfigurationen bei der zweidimensionalen Interaktion zwischen Stoß und Grenzschicht. Bei den Grundtypen handelt es sich um

- eine Rampe,

- einen einfallenden Stoß mit dessen Reflexion,

- eine stromauf gewandte Stufe und

- eine stromab gewandte Stufe.

Die jeweilige Strömungsstruktur ist in Abbildung 2.6 skizziert.

In dieser Arbeit wurde die Konfiguration mit einem einfallenden Verdichtungsstoß experimentell untersucht, weswegen sich die folgenden Beschreibungen auf diesen Fall fokussieren. Ein einfallender Verdichtungsstoß kann experimentell durch einen montierten Keil (bzw. angestellte Platte) erzeugt werden. Abhängig vom Anstellwinkel $\alpha$ der Stoßgenerator-Platte erfolgt eine entsprechende Ablenkung der Strömung, die mit der Ausbildung eines schrägen Verdichtungsstoßes einhergeht. Ein anliegender Verdichtungsstoß beginnt an der Spitze des Stoßgenerators und seine Intensität ist bei konstanter Machzahl alleine durch den Anstellwinkel definiert. Der einfallende Stoß wechselwirkt mit der Grenzschicht des Modells und erzeugt ein Stoßsystem mit einem reflektierten Stoß. Beim Stoßsystem wird unterschieden, ob durch den einfallenden Stoß eine Strömungsablösung auf der Modelloberfläche entsteht. Im Folgenden wird die Struktur des Stoßsystems mit bzw. ohne Ablösung erläutert. 


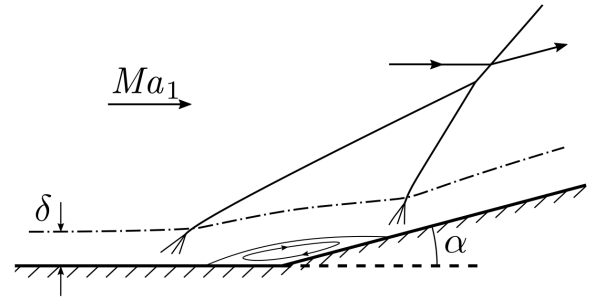

(a) Rampe

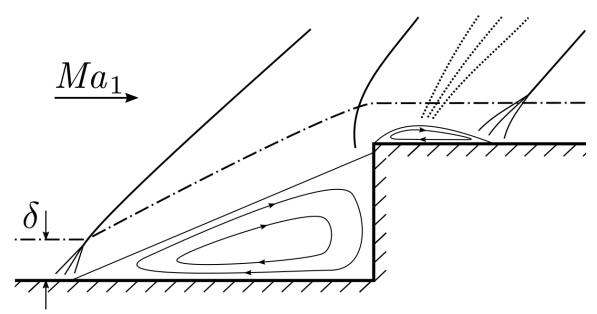

(c) stromauf gewandte Stufe

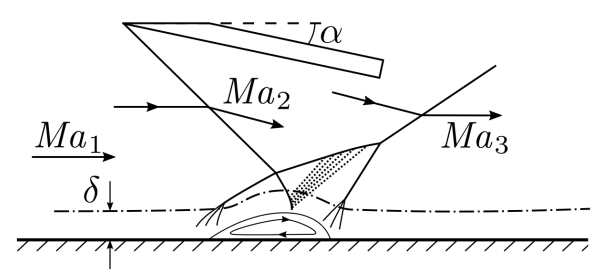

(b) einfallender Stoß mit Reflexion

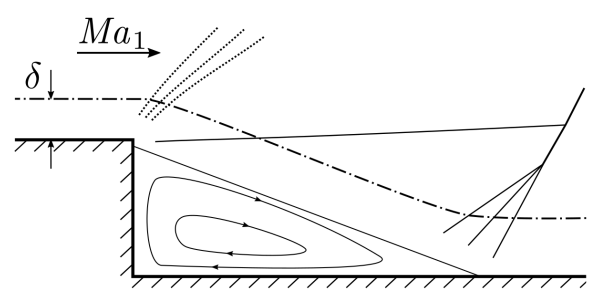

(d) stromab gewandte Stufe

Abbildung 2.6: Grundlegende Konfigurationen für 2D SGWW (nicht maßstabsgetreu)

\subsubsection{Schwache SGWW}

Abbildung 2.7 zeigt eine schematische Darstellung der Stoßstruktur für die Situation, dass der einfallende Stoß keine Ablösung verursacht. Mit dem Eintritt des einfallenden Stoßes in die Grenzschicht beginnt sich der Stoß aufgrund der abnehmenden Anströmmachzahl zu krümmen. Die detaillierten Vorgänge beim Durchqueren der Grenzschicht wurden von Henderson (1967) untersucht und hängen von den Anströmbedingungen ab. Mit zunehmender Eindringtiefe wächst zudem der Einfluss der viskosen Kräfte, wodurch die Stoßstruktur noch komplexer wird. Auf eine detaillierte Darstellung wird hier verzichtet, um den Fokus von den wesentlichen Vorgängen der SGWW nicht zu verlieren.

Durch den einfallenden Stoß entsteht ein Druckanstieg in Strömungsrichtung. Der Druckanstieg wirkt sich wegen des subsonischen Grenzschichtbereiches auch auf die Strömung stromauf des einfallenden Stoßes aus. Im subsonischen Bereich führt der Druckanstieg zu einer Verlangsamung der Strömung, was eine Ablenkung der Strömung und eine Aufdickung der Grenzschicht bedeutet. Aufgrund der Strömungsablenkung entstehen an der Schalllinie Kompressionswellen. Die Kompressionswellen werden beim Durchlaufen der Grenzschicht wegen der wachsenden Anströmmachzahl flacher und interagieren mit dem einfallenden Stoß. Schließlich laufen die Kompressionswellen zum reflektierten Stoß zusammen.

Im wandnahen Bereich wird die Strömung zunächst von der Oberfläche weggelenkt (Kompressionwellen) und stromab durch den einfallenden Stoß wieder in Richtung Oberfläche geleitet. Durch eine Abfolge weiterer Kompressionswellen wird die Strömung wieder tangential zur Modelloberfläche ausgerichtet.

Eine SGWW ohne Ablösung liegt nur bei schwachen Stoßintensitäten vor, wodurch die Ausdehnung der Stoßstruktur gering ist und üblicherweise vollständig innerhalb der Grenzschicht stattfindet. Von Weitem ähnelt die beschriebene Stoßstruktur dem Fall 


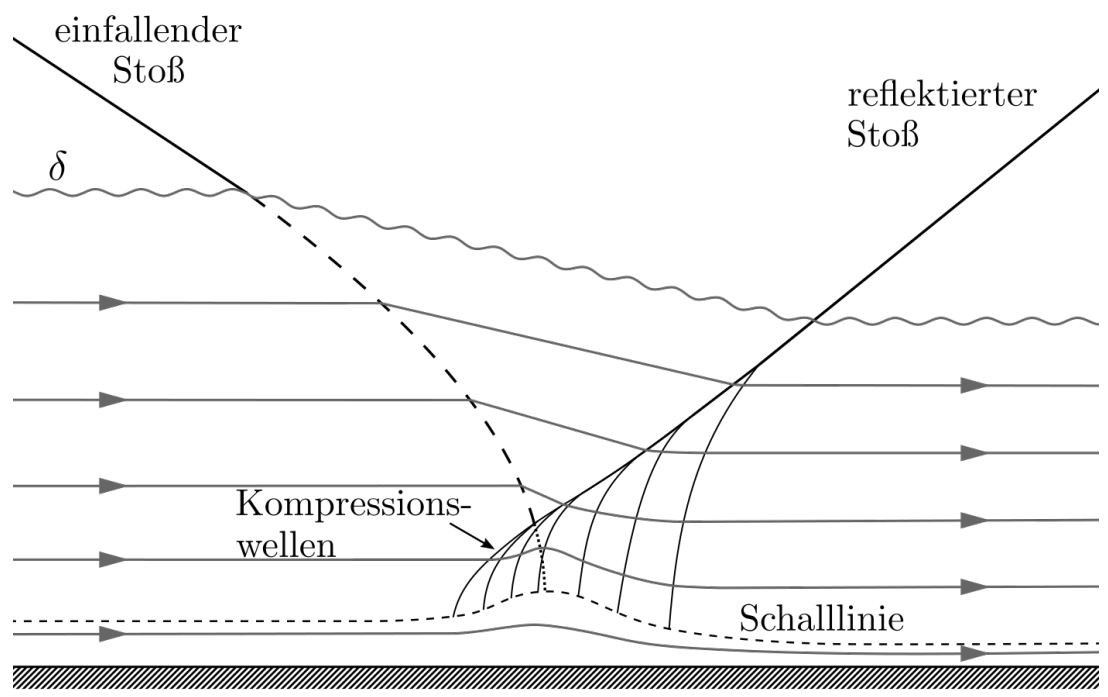

Abbildung 2.7: Strömungsstruktur eines einfallenden Stoßes ohne Ablösung

ohne Grenzschicht, bestehend aus der idealen Reflexion des einfallenden Stoßes. Diese Ähnlichkeit zeigt sich auch in der Druckverteilung auf der Plattenoberfläche (Abbildung 2.9a). Durch viskose Effekte der Grenzschicht kommt es zu einer räumlichen Glättung des Druckanstieges gegenüber der reibungsfreien Strömung. Nach einem steilen Anstieg an der Stoßauftreffstelle folgt eine stetige Annäherung an das Druckniveau ohne Grenzschicht. Babinsky \& Harvey (2011) zeigten anhand einer Rechnung auf Basis des Charakteristikenverfahrens, dass gasdynamische Relationen bei der Krümmung des einfallenden Stoßes zu dem verlangsamten Druckanstieg stromab der Auftreffposition führen. Da der Unterschied im Druckverlauf im Vergleich zum reibungsfreiem Fall gering ausfällt, spricht man bei der SGWW ohne Ablösung auch von einer schwachen Interaktion.

\subsubsection{Starke SGWW}

Ist der stoßinduzierte Druckanstieg in der Grenzschicht ausreichend stark, löst die Grenzschicht von der Wand ab und die Strömungstopologie verändert sich. Eine Skizze des zu erwartenden Stoßsystems mit Ablösung ist in Abbildung 2.8 gezeigt.

Analog zur schwachen SGWW beginnt die Entstehung des Stoßsystems mit dem Eintritt des einfallenden Stoßes in die Grenzschicht, der damit einhergehenden Krümmung des Stoßes und der Erzeugung eines positiven Druckgradienten. Die Grenzschicht löst an dem Punkt S ab, der aufgrund des subsonischen Grenzschichtbereiches stromauf von der Stoßauftreffposition liegt. Das Verhalten einer Überschallströmung bei einer ausreichend großen Ablösung kann nach Chapman et al. (1957) als freie Interaktion betrachtet werden. Dies bedeutet, die Strömung am Ablösepunkt hängt ausschließlich von den lokalen Strömungsbedingungen ab. Als Konsequenz ist die Ablösung unabhängig von der Ursache des Druckanstiegs, was bei geeigneter Skalierung einen Vergleich zwischen verschiedenen Typen von SGWW ermöglicht. 


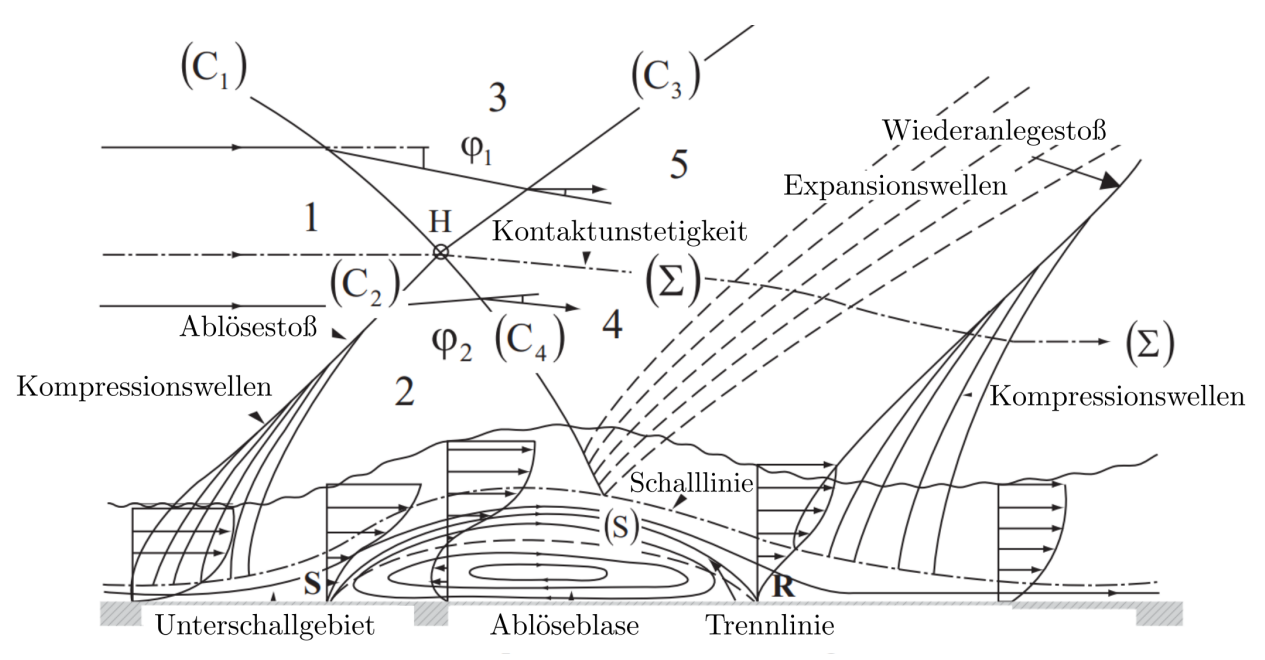

Abbildung 2.8: Strömungsstruktur eines einfallenden Stoßes mit Ablösung (übersetzt aus Babinsky \& Harvey (2011))

Das Ablösegebiet führt zu einer Ablenkung der Anströmung, wodurch im Überschallgebiet der Grenzschicht Kompressionswellen stromauf von $S$ entstehen. Die Kompressionswellen durchlaufen die Grenzschicht und bilden den Ablösestoß $\left(C_{2}\right)$. Da der Ablösepunkt signifikant stromauf von der Stoßauftreffposition liegt, befindet sich der Interaktionspunkt $H$ zwischen dem einfallenden Stoß $\left(C_{1}\right)$ und den Ablösestoß $\left(C_{2}\right)$ normalerweise außerhalb der Grenzschicht. Hierbei handelt es sich um eine Stoß-Stoß-Interaktion, aus der sich die Stoßwinkel von $C_{3}$ und $C_{4}$ ergeben. Da sich die Entropieerhöhungen über die Stöße $C_{1}$ und $C_{3}$ von der Variante über $C_{2}$ und $C_{4}$ unterscheiden, ist $H$ Ausgangspunkt einer Kontaktunstetigkeit.

Die Kontaktlinie zwischen der abgelösten Strömung und dem Fluid innerhalb der Ablöseblase wird als Trennlinie bezeichnet. Quer zur Trennlinie bildet sich eine Scherschicht aus. Aufgrund des Impulsaustausches findet innerhalb der Ablöseblase eine Rezirkulation statt und die Strömung entlang der Trennlinie erhöht ihren Impuls. Die Lage des Ablösepunktes verschiebt sich solange stromauf, bis der Impuls an der Trennlinie ausreicht, um den stoßinduzierten Druckanstieg zu überwinden (Needham \& Stollery 1966). Der einfallende Stoß $\left(C_{4}\right)$ lenkt die Strömung in Richtung Oberfläche und die abgelöste Scherschicht legt sich wieder an die Modelloberfläche an. Der Abstand zwischen dem Ablöse- $(S)$ und Wiederanlegepunkt $(R)$ definiert die Länge des Ablösegebietes.

Die Umlenkung der Strömung in Richtung Modelloberfläche erzeugt weitere Kompressionswellen stromab von $R$. Durch die Kompressionswellen findet eine letzte Umlenkung der Strömung statt, sodass die Strömung parallel zur Oberfläche verläuft. Die Kompressionswellen laufen zusammen und bilden den Wiederanlegestoß.

Eine schematische Darstellung des Oberflächendruckes entlang der Strömungsrichtung ist in Abbildung 2.9b dargestellt. Die Verteilung weicht deutlich vom reibungsfreien Fall ab. Stromauf der nicht-viskosen Stoßauftreffposition findet aufgrund der Ablösung ein erster Druckanstieg statt. Innerhalb der Ablöseblase liegt ein nahezu konstanter Plateaudruck vor. Im Bereich des Wiederanlegepunktes kommt es zu einem zweiten 
rapiden Druckanstieg, hervorgerufen durch den einfallenden Verdichtungsstoß $C_{4}$ und den Wiederanlegestoß.

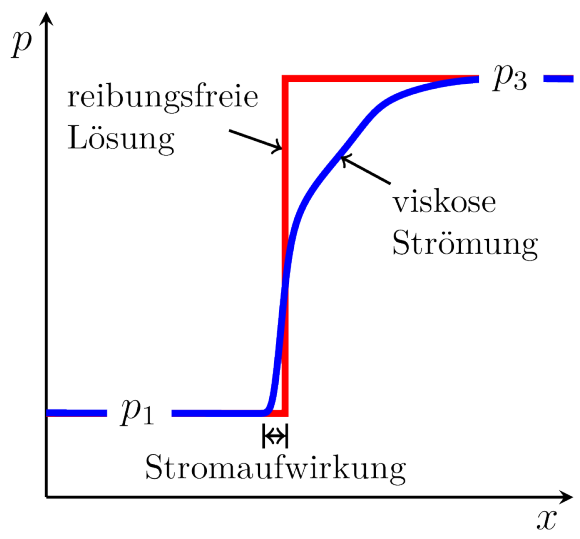

(a) Schwache SGWW ohne Ablösung

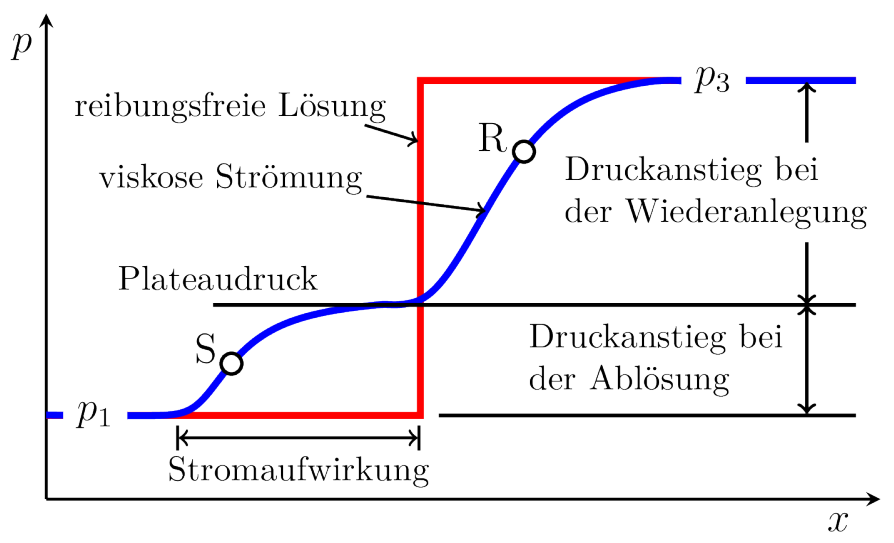

(b) Starke SGWW mit Ablösung

Abbildung 2.9: Druckverteilungen auf der Modelloberfläche aufgrund einfallender SGWW

Die Höhe des Plateaudrucks hängt nach der freien Interaktions-Theorie nur davon ab, wie widerstandsfähig die Grenzschicht gegenüber dem Druckanstieg am Ablösepunkt ist. Folglich führt ein stärkerer einfallender Stoß in erster Linie zu einem höherem Level des zweiten Druckanstiegs. Um den stärkeren zweiten Druckanstieg zu überwinden, benötigt die abgelöste Strömung mehr Impuls, wodurch der Ablösepunkt stromauf wandert und die Länge der Ablöseblase zunimmt. Durch die Verschiebung des Ablösepunktes verringert sich die dortige Grenzschichtdicke und es ist eine geringe Erhöhung des Plateaudrucks zu erwarten.

\subsubsection{Grenzschichtzustand bei SGWW}

Neben der Stoßintensität hängt die Strömungstopologie auch maßgeblich vom Grenzschichtzustand ab. Laminare Grenzschichten tendieren aufgrund des geringeren Impulsaustausches bereits bei kleineren Druckanstiegen zur Ablösung als turbulente Grenzschichten. Als Konsequenz ist bei laminaren Grenzschichten das Ablösegebiet im Allgemeinen größer. Bei beiden Grenzschichtzuständen wächst die Größe des Ablösegebietes mit der Grenzschichtdicke am Ablösepunkt, da sich weniger Impuls in Wandnähe befindet. Transitionelle SGWW stellen bezüglich der Größe der Ablöseblase vereinfacht betrachtet einen Übergang zwischen den beiden Niveaus dar (Needham \& Stollery 1966).

Die SGWW beeinflussen allerdings auch den Verlauf der natürlichen Transition. Pagella et al. (2002) zeigten anhand von direkten numerischen Simulationen und linearen Stabilitätsanalysen, dass Instabilitäten durch die SGWW verstärkt anwachsen. Zusätzlich werden durch die SGWW weitere Störungen in die Grenzschicht eingebracht. Beide Effekte können den laminar-turbulenten Umschlag auslösen oder bei bereits transitionellen SGWW den Prozess signifikant beschleunigen. Krishnan \& Sandham (2007) untersuchten numerisch wie sich ein Turbulenzfleck beim Passieren einer einfallenden SGWW 
entwickelt und wiesen ein starkes Wachstum nach, was einem beschleunigten Transitionsprozess entspricht. Stromab des Wiederanlegepunktes führt die angeregte Transition zu hohen Wärmelasten, die bislang noch nicht a priori abgeschätzt werden können. 



\section{Messaufbau}

In diesem Kapitel wird die technische Umsetzung der Experimente vorgestellt. Hierbei wird sowohl der verwendete Windkanal als auch das Testmodell beschrieben. Daran anschließend werden die eingesetzten Messverfahren vorgestellt und die Aufbereitung der Messdaten erläutert. Zum Abschluss des Kapitels wird der Umfang der durchgeführten Parameterstudie geschildert.

\subsection{Rohrwindkanal Göttingen}

Die Windkanalmessungen wurden am Rohrwindkanal Göttingen (RWG) (Ludwieg 1955 , Ludwieg et al. 1969) durchgeführt. Eine Skizze des Windkanals ist in Abbildung 3.1 dargestellt. Mit der Anlage können Machzahlen im Bereich $2 \leq M a \leq 7$ und Einheitsreynoldszahlen im Bereich $2 \cdot 10^{6} \mathrm{~m}^{-1} \leq R e_{1} \leq 110 \cdot 10^{6} \mathrm{~m}^{-1}$ untersucht werden. Detailliertere Informationen bezüglich der untersuchbaren Strömungsparameter sind in Tabelle 3.1 aufgeführt.

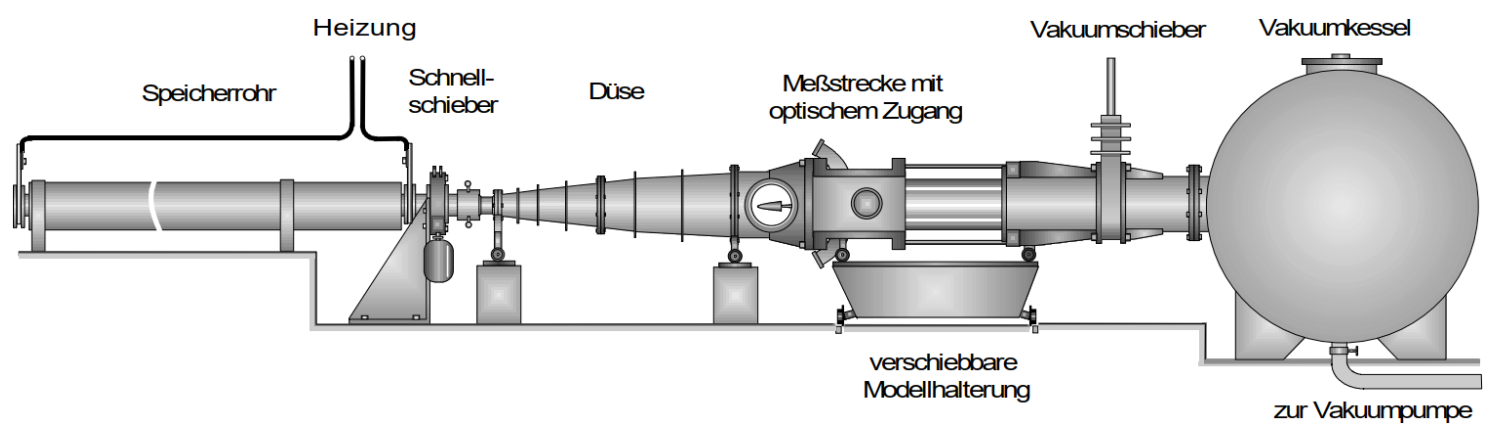

Abbildung 3.1: Skizze der Ludwieg-Rohr Anlage RWG am DLR Göttingen (Schülein 1999)

Die Anlage basiert auf dem Prinzip des Ludwieg-Rohrs und kann grob in zwei Bereiche unterteilt werden. Im Speicherrohr wird vor dem Versuch der Ruhedruck $p_{0}$ auf den gewünschten Wert eingestellt und das Gas falls nötig erhitzt. Das Speicherrohr ist über einen Schieber von dem Niederdruckbereich der Anlage getrennt. Auf der anderen Seite des Schiebers befindet sich die Lavaldüse, die Messstrecke sowie ein Vakuumtank. In allen drei Komponenten wird der Druck vor dem Versuch auf circa 55 mbar reduziert, um ein ausreichendes Druckverhältnis für den Betrieb der Lavaldüse zu gewährleisten.

Die Messung wird eingeleitet durch das Öffnen des Schiebers. Hierdurch breitet sich ein Verdichtungsstoß in das druckreduzierte Gebiet aus und Expansionswellen laufen 


\begin{tabular}{l|ccc|ccc}
\hline$M a$ & 2 & 3 & 4 & 5 & 6 & 6.85 \\
Rohr & A & A & A & B & B & B \\
$p_{0}[\mathrm{MPa}]$ & $0.05-0.7$ & $0.06-0.6$ & $0.08-1.0$ & $0.4-2.9$ & $0.4-3.4$ & $0.4-3.6$ \\
$T_{0}[\mathrm{~K}]$ & $236-262$ & $241-267$ & $258-287$ & $340-610$ & $410-640$ & $440-655$ \\
$R e_{1}\left[10^{6} \mathrm{~m}^{-1}\right]$ & $10-110$ & $6-70$ & $4-60$ & $5-55$ & $3-28$ & $2-17$ \\
Messstrecke & $0.34 \times 0.35 \mathrm{~m}^{2}$ & $0.5 \times 0.5 \mathrm{~m}^{2}$ & $0.5 \times 0.5 \mathrm{~m}^{2}$ & $\varnothing 0.5 \mathrm{~m}$ & $\varnothing 0.5 \mathrm{~m}$ & $\varnothing 0.5 \mathrm{~m}$ \\
\hline
\end{tabular}

Tabelle 3.1: Strömungsparameterbereich des Rohrwindkanals Göttingen

durch das Speicherrohr, wobei das ruhende Testgas in Richtung Messstrecke beschleunigt wird. Nach einer Einlaufphase baut sich die Strömung auf und es liegen quasistationäre Stömungsbedingungen in der Messstrecke vor. Die Messzeit endet, sobald die ersten Expansionswellen am Ende des Speicherrohres reflektieren und erneut die Lavaldüse erreichen. Der RWG besitzt zwei verschiedene Speicherrohre, ein ungeheiztes Rohr A und ein heizbares Rohr B, mit einer jeweiligen Länge von $80 \mathrm{~m}$. Aus der Länge ergibt sich eine Messzeit von 300 ms-400 ms, abhängig von der Temperatur und der Ausströmgeschwindigkeit im Speicherrohr.

Die Experimente in dieser Arbeit wurden bei einer nominellen Machzahl von $M a=6$ durchgeführt. Die exakte Machzahl der verwendeten Düse beträgt nach Krogmann (1995) $M a=5.98 \pm 0.06$. Durch eine Anpassung des Totaldrucks $p_{0}$ wurde die Einheitsreynoldszahl zwischen $R e_{1}=8 \cdot 10^{6} \mathrm{~m}^{-1}$ und $R e_{1}=24 \cdot 10^{6} \mathrm{~m}^{-1}$ variiert, wodurch sich der transitionelle Zustand der Grenzschicht an einer festen Position verändert. Die Totaltemperatur wurde für alle Messungen zum Zeitpunkt $200 \mathrm{~ms}$ nach der Schieberöffnung möglichst konstant bei $T_{0}=543 \mathrm{~K} \pm 5 \mathrm{~K}$ gehalten. In Tabelle 3.2 sind representative Werte für die relevanten Strömungsparameter bei den untersuchten Einheitsreynoldszahlen angegeben.

\begin{tabular}{ccccccc}
\hline$R e_{1}\left[10^{6} \mathrm{~m}^{-1}\right]$ & $p_{0}[\mathrm{MPa}]$ & $T_{0}[\mathrm{~K}]$ & $p_{\infty}[\mathrm{Pa}]$ & $T_{\infty}[\mathrm{K}]$ & $\rho_{\infty}\left[\mathrm{kg} / \mathrm{m}^{3}\right]$ & $u_{\infty}[\mathrm{m} / \mathrm{s}]$ \\
\hline 8 & 1.08 & 543 & 700 & 66.6 & 0.037 & 978 \\
14 & 1.89 & 543 & 1220 & 66.6 & 0.064 & 978 \\
19 & 2.55 & 543 & 1650 & 66.6 & 0.086 & 978 \\
24 & 3.32 & 543 & 2090 & 66.6 & 0.109 & 978 \\
\hline
\end{tabular}

Tabelle 3.2: Repräsentative Strömungsparameter bei den untersuchten Einheitsreynoldszahlen am RWG

Bei jeder Messung am RWG wird der Totaldruck $p_{0}$ und die Totaltemperatur $T_{0}$ stromauf der Lavaldüse aufgezeichnet. Diese Messdaten werden verwendet um die Strömungsparamter in der Messstrecke anhand isentroper Zustandsänderungen zu berechnen. Mit Hilfe des Sutherland-Gesetzes (Gl. 2.7) kann von dem Zustand im Speicherrohr auf die Einheitsreynoldszahl in der Messstrecke geschlossen werden. Auf Basis von 44 einzelnen 
Versuchen zeigt Abbildung 3.2 den gemittelten Verlauf der Windkanaldaten zusammen mit der $1 \sigma$-Umgebung.
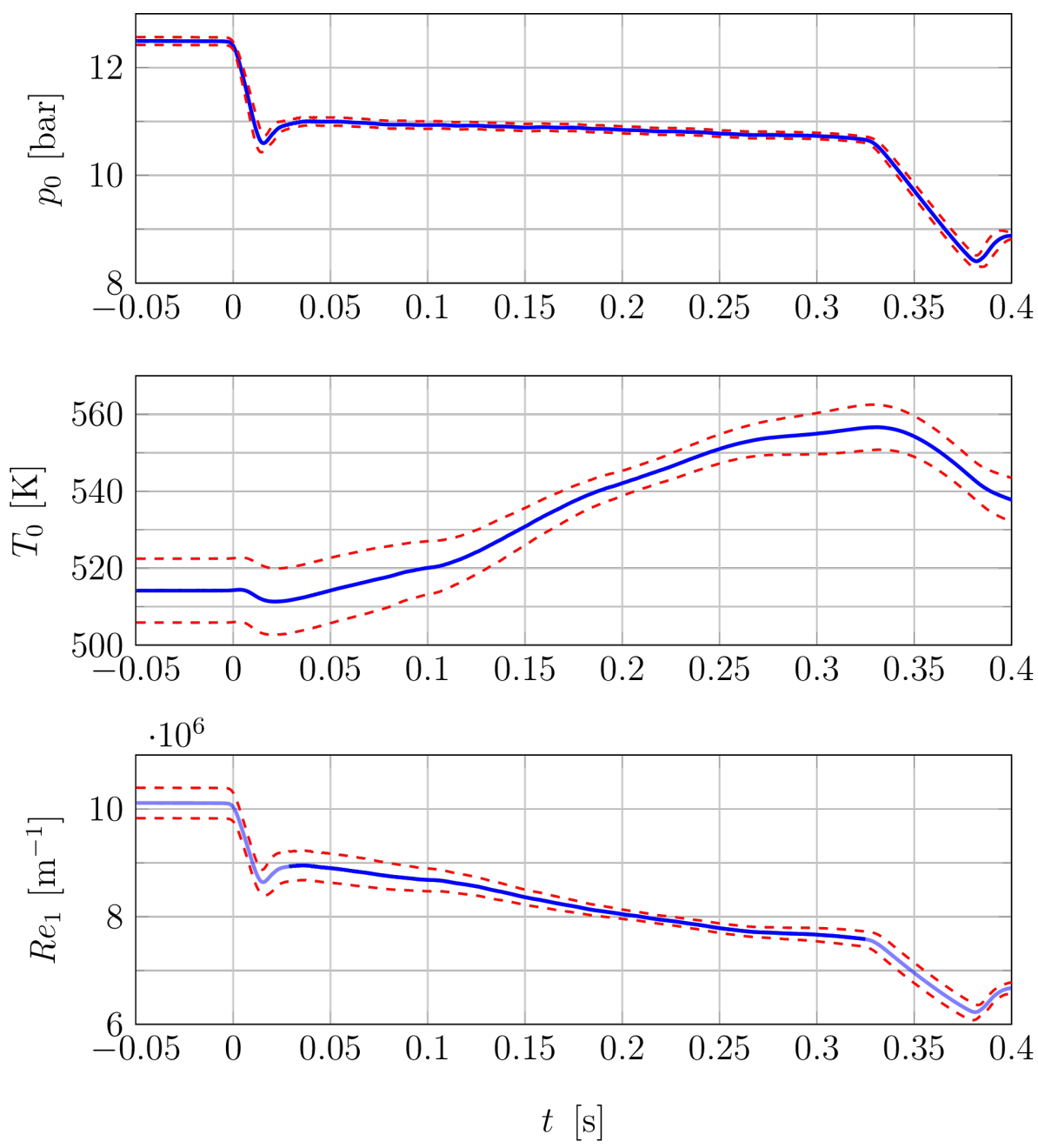

Abbildung 3.2: Über die Messzeit sind der Totaldruck und die Totaltemperatur stromauf der Lavaldüse dargestellt. Zusätzlich ist die daraus berechnete Einheitsreynoldszahl in der Messstrecke aufgetragen. Die Kurven sind aus 44 einzelnen Versuchen gemittelt und die jeweilige Streuung in Form der $1 \sigma$-Umgebung durch gestrichelte Linien angezeigt.

Die verschiedenen Phasen der Messung sind am deutlichsten anhand des Totaldrucks zu erkennen. Bei $t=0 \mathrm{~s}$ beginnt die Öffnung des Schiebers und nach ca. $30 \mathrm{~ms}$ liegen quasi-stationäre Bedingungen vor. Bei $t \approx 330 \mathrm{~ms}$ erreichen die Expansionswellen die Lavaldüse und beenden die Messzeit. Die Temperatur stromauf der Lavaldüse steigt während der Messzeit, da das Gas im Speicherrohr nicht auf eine einheitliche Temperatur aufgeheizt werden kann. Der Temperaturgradient verändert sich im Verlauf des Messtages, weshalb die Streuung beim Temperaturverlauf größer ausfällt. Während der quasi-stationären Messzeit kommt es aufgrund der steigenden Temperatur zu einer steti- 
gen Reduzierung von $R e_{1}$. Falls bei einer Messtechnik eine zeitliche Mittelung der Daten durchgeführt wird, so erfolgt diese Mittelung symmetrisch um die gewünschte Einheitsreynoldszahl über den Zeitbereich $\pm 50 \mathrm{~ms}$. Die gewünschte Einheitsreynoldszahl verändert sich während dieses Mittelungszeitraums um circa $\pm 4 \%$.

\subsection{Modell}

Das untersuchte Modell ist in Abbildung 3.3 dargestellt. Das Modell besteht aus einer ebenen Basisplatte mit eine Länge von $669 \mathrm{~mm}$ und Breite von $400 \mathrm{~mm}$. Der Vorderkantenradius beträgt $0.1 \mathrm{~mm}$ und stellt einen Kompromiss zwischen der laminaren Lauflänge, der Stärke des Vorderkantenstoßes und der realisierbaren Fertigungstoleranz dar. Der spannweitige Mittelpunkt der Vorderkante wurde als Ursprung für das verwendete Koordinatensystem gewählt (siehe Abbildung 3.3). Die ebene Platte besitzt eine $150 \mathrm{~mm}$ breite rechteckige Aussparung zwischen $x=96 \mathrm{~mm}$ und $x=496 \mathrm{~mm}$. Die Aussparung ermöglicht die oberflächenbündige Montage spezieller Einsätze zur Messung diverser Strömungsparameter. Die Details der einzelnen Einsätze werden zusammen mit der jeweiligen Messtechnik beschrieben. An den Kanten der Aussparung befinden sich mit Spachtelmasse aufgefüllte Sacklöcher $(\varnothing 2 \mathrm{~mm})$, die bei optischen Messtechniken als Referenzpunkte genutzt werden.

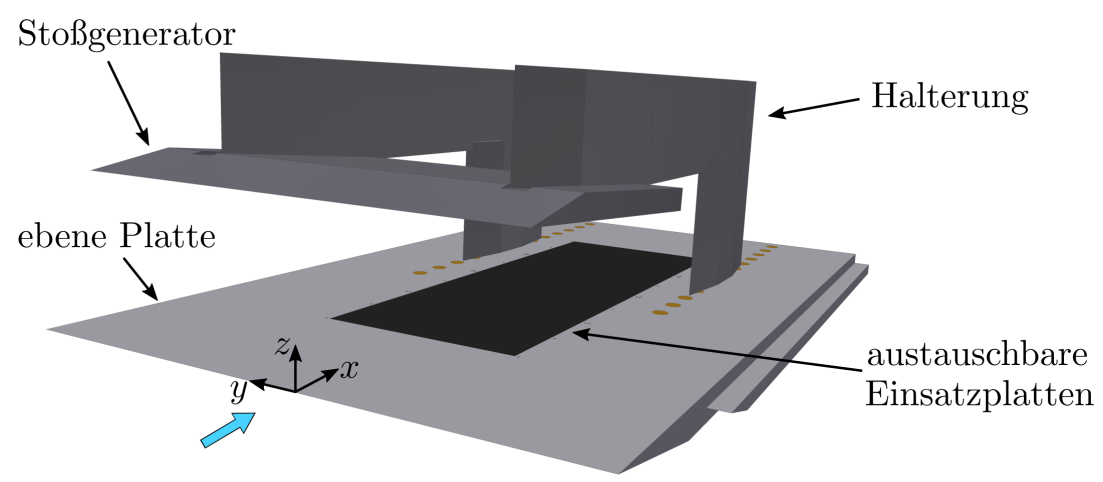

Abbildung 3.3: Skizze des Windkanalmodells bestehend aus einer ebenen Platte mit montiertem Stoßgenerator. In der Aussparung ist der schwarze PlexiglasEinsatz dargestellt

Optional kann oberhalb der ebenen Platte ein Stoßgenerator in Form eines Keils über Halterungen mit der Platte verschraubt werden. Der Stoßgenerator ist insgesamt $300 \mathrm{~mm}$ breit und $255 \mathrm{~mm}$ lang, wobei eine Verlängerung $(45 \mathrm{~mm})$ bündig an das Ende des Stoßgenerators installiert wurde, um die genannte Gesamtlänge zu erreichen. Durch die modulare Bauweise der Halterung wurden folgende Parameter bezüglich des Stoßgenerators verändert. Der Anstellwinkel der Stoßgeneratorplatte wurde zwischen den Werten $\alpha=2^{\circ}, 3^{\circ}$ und $4^{\circ}$ variiert, um unterschiedliche Stoßintensitäten zu erzeugen. In Tabelle A.2 sind die Strömungsparamter stromab des einfallenden bzw. reflektierten Stoßes auf Basis der schrägen Stoßrelationen für die drei Ablenkwinkel aufgeführt. 
Durch eine Vielzahl an Bohrlöchern (siehe Abbildung 3.3) kann die Position des Stoßgenerators in Strömungsrichtung in $15 \mathrm{~mm}$ Schritten verschoben werden, wodurch sich die Auftreffposition des erzeugten Verdichtungsstoßes im Bezug zur Plattenvorderkante ändert. Insgesamt gibt es 27 Positionen für die Montage der Halterung. Für die am weitesten stromauf gelegene Position (Pos. I) zeigt Tabelle 3.3 die Lage der Stoßgeneratorspitze im Koordinatensystem der ebenen Platte. Die z-Position der Stoßgeneratorspitze wurde für jeden Anstellwinkel angepasst mit dem Ziel, an einer gegeben Position für alle Anstellwinkel die selbe Stoßauftreffposition zu erhalten. Die Anpassung der z-Position wurde anhand von nicht-viskosen Rechnungen durchgeführt.

\begin{tabular}{ccc}
\hline Anstellwinkel $\left[^{\circ}\right]$ & $x[\mathrm{~mm}]$ & $z[\mathrm{~mm}]$ \\
\hline 2 & -165.00 & 58.24 \\
3 & -165.00 & 62.13 \\
4 & -165.00 & 66.27 \\
\hline
\end{tabular}

Tabelle 3.3: Koordinaten der Stoßgeneratorspitze für die maximal stromauf gelegene Stoßgeneratorposition (Pos. I).

Als weitere Option kann noch die Höhe des Stoßgenerators durch ein Distanzbauteil um $10 \mathrm{~mm}$ erhöht werden. Diese Möglichkeit war für die Durchführung der Messungen notwendig, wie in Abbildung 3.4 anhand zweier Messpositionen verdeutlicht wird.


Abbildung 3.4: Vereinfachtes Stoßsystem des Modells. Die Skizze zeigt sowohl den gewünschten Verlauf (grün) des Vorderkantenstoßes als auch einen unerwünschten Verlauf (rot) mit der Stoßreflexion an der Unterseite des Stoßgenerators

Abbildung 3.4 zeigt eine stark vereinfachte Darstellung des zu erwartenden Stoßsystems für zwei Modellkonfigurationen. Befindet sich der Stoßgenerator im Bezug zur Vorderkante der ebenen Platte ausreichend stromauf (linke Skizze), so verläuft der Vorderkantenstoß unterhalb des Stoßgenerators. Andernfalls reflektiert der Vorderkantenstoß am Stoßgenerator (rechte Skizze), trifft auf die ebene Platte und beeinflusst das Messgebiet. Wenn die Vorderkante des Stoßgenerators entlang der jeweiligen virtuellen Stoßfrontlinie (blau gestrichelte Linie) diagonal stromauf verschiebbar wäre, könnte das Problem an jeder erwünschten Stoßauftreffposition umgangen werden. Aus diesem Grund wurde in der vorliegenden Studie die erwähnte zweite vertikale Position des Stoßgenerators vorgesehen. 


\subsection{Schattenverfahren}

Schattenverfahren gehören zusammen mit den eng verwandten Schlierenverfahren zu den wichtigsten und am häufigsten praktizierten Messtechniken im Bereich der Überschallaerodynamik. Beide Messverfahren basieren darauf, dass ein paralleler Lichtstrahl durch die Messstrecke verläuft und aufgrund eines Gradienten im Brechungsindex abgelenkt werden. Über die Gladstone-Dale Beziehung (Gladstone \& Dale 1863) besteht ein linearer Zusammenhang zwischen dem Brechungsindex eines Gases und dessen Dichte. Da die Dichte in Überschallströmungen durch Verdichtungsstöße und Expansionswellen beeinflusst wird, können Schatten- beziehungsweise Schlierenaufnahmen die Strömungstopologie (insbesondere die Stoßstruktur) visualisieren. Detaillierte Informationen zur Funktionsweise und Umsetzung der beiden Messtechniken sind in dem Buch von Settles (2001) beschrieben. Die folgenden Erläuterungen fokussieren sich auf die in dieser Arbeit durchgeführten fokussierten Schattenaufnahmen.

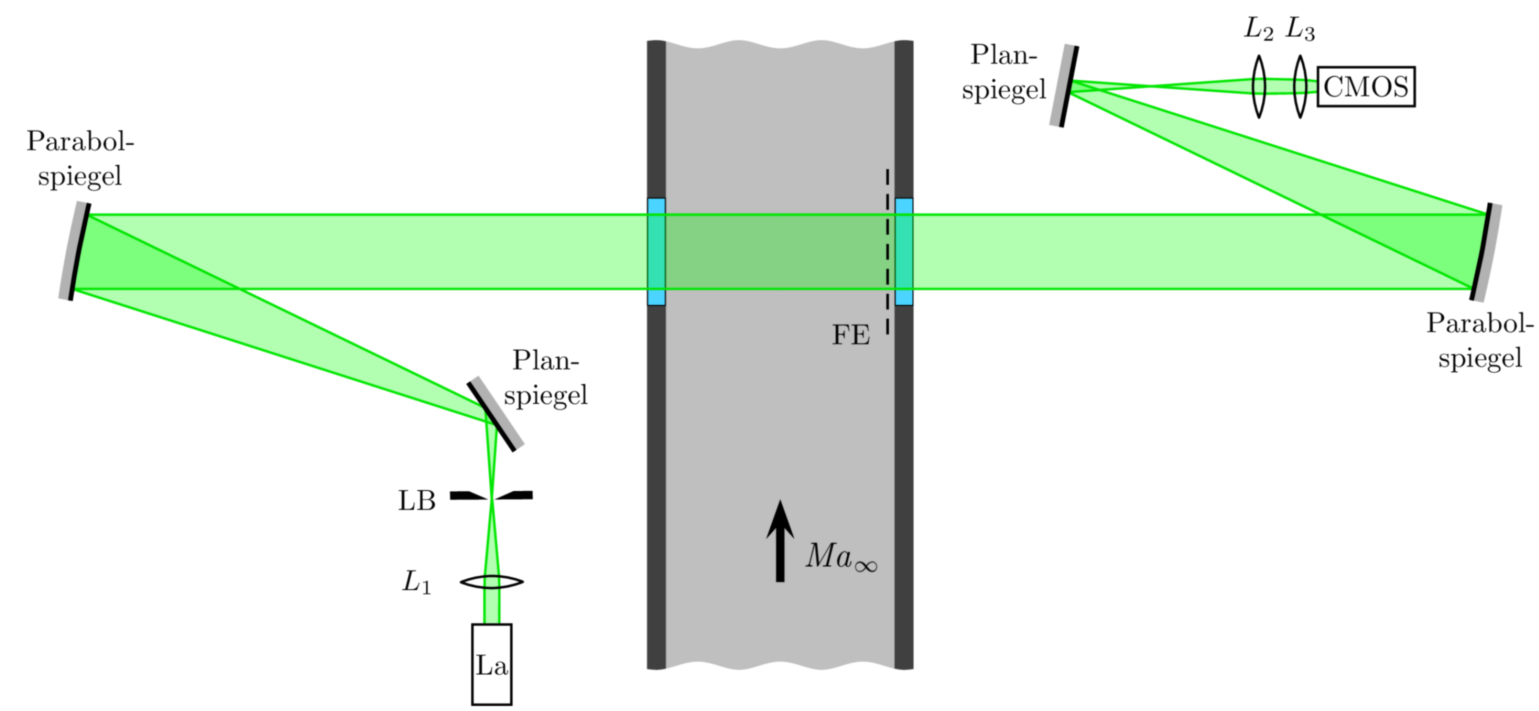

Abbildung 3.5: Skizze des Messaufbaus der fokussierten Schattenaufnahmen am RWG

Abbildung 3.5 zeigt eine Skizze vom optischen Aufbau am RWG. Zunächst wird der Laserstrahl mittels einer Linse $\left(L_{1}\right)$ fokussiert, sodass sich der Fokuspunkt im Brennpunkt des nachfolgenden Parabolspiegels befindet. Eine Lochblende (LB) entfernt unerwünschte Lichtstrahlen aus der Brennebene und erzeugt eine idealisierte Punktquelle. Die passierten Lichtstrahlen reflektieren an einem Planspiegel und treffen anschließend auf den Parabolspiegel. Da sich die Punktquelle in der Brennebene des Parabolspiegels befindet, entsteht ein paralleler Lichtstrahl, der durch die Messstrecke verläuft. Nach dem Passieren der Messstrecke wird der Lichtstrahl von einem zweiten Parabolspiegel gebündelt und zwei Linsen $\left(L_{2}, L_{3}\right)$ fungieren als Objektiv für den CMOS-Sensor der Kamera. In den Messungen wurde als Fokusebene (FE) die Windkanalgrenzschicht der Empfängerseite gewählt, um die Lichtbrechung in dieser Ebene auszublenden. Als Lichtquelle wurde ein Cavilux Smart $(400 \mathrm{~W})$ Laser der Firma Cavitar Ltd verwendet. Der Laser wurde im Pulsbetrieb bei einer Frequenz von $2 \mathrm{kHz}$ und einer Pulsbreite von $20 \mathrm{~ns}$ 
betrieben. Als Kamera kam eine pco.dimax HS4 der Firma PCO AG zum Einsatz. Die Kamera erfasste die Bilder in einer Auflösung von $2000 \times 1000$ Pixel bei einer Aufnahmerate von $2 \mathrm{kHz}$. Während der Belichtungszeit bewegt sich das Fluid in der Anströmung um $20 \mu \mathrm{m}$ (entspricht 0.14 Pixel), sodass eine Momentaufnahme der Strömung mit einer geringen Bewegungsunschärfe entsteht. Mit diesen Einstellungen wurden bei jeder Messung circa 800 Bilder aufgenommen, die den Aufbau der Strömung, die Strömungsstruktur während der Messzeit und den Strömungszusammenbruch zeigen.

Durch die Brechungsindex-Verteilung innerhalb der Messstrecke wird der Lichtstrahl abgelenkt. Die Schattenmesstechnik ist allerdings nur sensitiv auf die zweite Ableitung des Brechungsindex, denn ein konstanter Gradient des Brechungsindex führt zu einer einheitlichen Ablenkung der Lichtstrahlen, wodurch im Bild keine Intensitätsunterschiede sichtbar werden. Der Kontrast in den Schattenaufnahmen hängt nicht nur von dem Betrag der zweiten Ableitung ab. Da das parallele Strahlenbündel durch die gesamte Messstrecke verläuft, wird die Ablenkung des Lichtstrahls über die Breite der Messstrecke integriert. Als Konsequenz erscheinen zweidimensionale Effekte, die sich über die gesamte Breite erstrecken, kontraststärker als lokale Dichteänderungen.

In den Schattenaufnahmen konnte mittels transparentem Millimeterpapier eine tonnenförmige Verzeichnung festgestellt werden, die daraufhin korrigiert wurde. Zusätzlich existieren vibrationsbedingte Verschiebungen zwischen den einzelnen Schattenaufnahmen, die durch den Windkanalbetrieb verursacht werden. Die Verschiebung beträgt bis zu 7 Pixel (entspricht im Bild ca. $1 \mathrm{~mm}$ ) und wurde korrigiert, indem die Einzelbilder zunächst durch einen bilateralen Filter (Tomasi \& Manduchi 1998) aufbereitet und anschließend eine Kantendetektion mit dem Canny-Algorithmus (Canny 1986) durchgeführt wurde. Die Einstellungsparameter für beide Methoden wurden so gewählt, dass die Modellkanten im Schattenbild zuverlässig detektiert wurden. Mit einer Kreuzkorrelation wurde die Verschiebung der Einzelbilder anhand der Modellkanten berechnet und die Position der Rohbilder korrigiert. Anhand zweier Referenzpunkte im Bild erfolgt anschließend eine lineare Umrechnung der Pixel-Koordinaten in das Koordinatensystem der ebenen Platte.

Ein Ziel der Schattenaufnahmen ist die Bestimmung der nicht-viskosen Auftreffposition $x_{\text {imp }}$ des einfallenden Verdichtungsstoßes. Die Auftreffposition ergibt sich anhand des Schnittpunktes zwischen der Plattenoberfläche und der virtuell verlängerten Stoßfront. Für die Bestimmung wurde erneut der bilaterale Filter und die Kantendetektion verwendet. Diesmal wurden die Einstellungsparameter so gewählt, dass der einfallende Verdichtungsstoß detektiert wird. Der Verlauf des Verdichtungsstoßes wird als Gerade angenommen und auf Basis einer linearen Regression erfolgt die Bestimmung des Winkels und die Auftreffposition des Stoßes. Ein Beispiel für ein ausgewertetes Schattenbild ist in Abbildung 3.6 dargestellt.

\subsection{Quantitative Infrarotthermographie}

Die Verteilung der Wärmestromdichte auf der ebenen Platte wurde mittels Infrarotthermographie gemessen. Hierfür wurde ein Einsatz aus Plexiglas ${ }^{\circledast}$ bündig in die Aussparung 


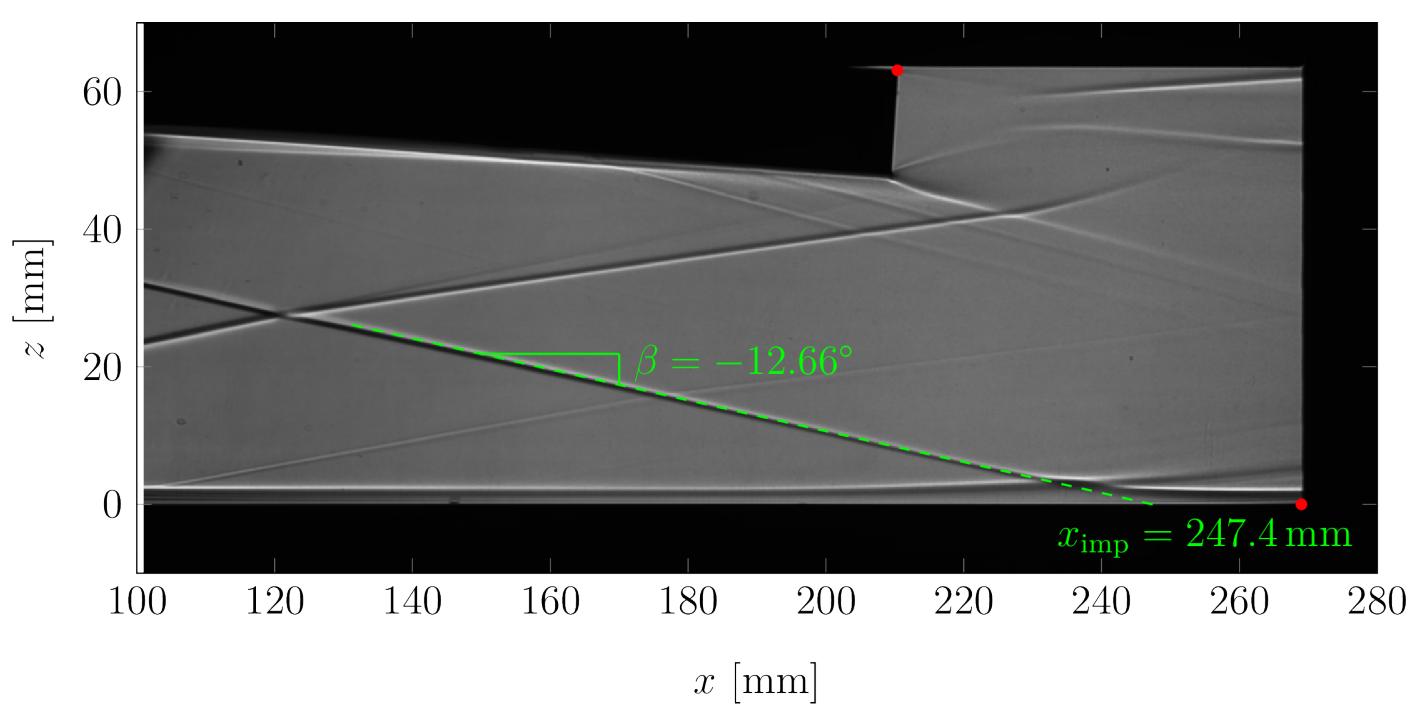

Abbildung 3.6: Beispiel für die Auswertung einer Schattenaufnahme. Markiert sind die beiden Referenzpunkte (rote Punkte) für die Umrechnung ins Koordinatensystem der ebenen Platte und die Lage des Verdichtungsstoßes samt Auftreffposition

der Basisplatte eingebaut (siehe Abbildung 3.3). Relevante Stoffgrößen vom Plexiglas ${ }^{\circledR}$ sind in Tabelle 3.4 aufgelistet. Die Emissivität der Plexiglasoberfläche wurde indirekt unter der Voraussetzung $\varepsilon_{\text {Plexi }}=1-r_{\text {Plexi }}$ bestimmt, wobei $r_{\text {Plexi }}$ die Reflektivität der Oberfläche angibt. Im Rahmen einer Kalibrierungsmessung wurden verschiedene Hintergrundtemperaturen mit einem Schwarzkörperstrahler vorgegeben. Die von der Plexiglasoberfläche reflektierte Strahlung wurde mittels Infrarotkamera detektiert und zur Bestimmung von $r_{\text {Plexi }}$ verwendet.

\begin{tabular}{ll}
\hline Wärmeleitfähigkeit & $\lambda_{\text {Plexi }}=0.202 \mathrm{~W} / \mathrm{Km}$ \\
spezifische Wärmekapazität & $c_{p \text {,Plexi }}=1.48 \mathrm{~kJ} / \mathrm{kgK}$ \\
Dichte & $\rho_{\text {Plexi }}=1183 \mathrm{~kg} / \mathrm{m}^{3}$ \\
Emissivität & $\varepsilon_{\text {Plexi }}=0.96$ \\
\hline
\end{tabular}

Tabelle 3.4: Relevante Stoffgrößen des Plexiglas-Einsatzes bei $T_{\mathrm{w}}=300 \mathrm{~K}$

Die geringe Wärmeleitfähigkeit reduziert den räumlichen Temperaturausgleich sowohl an der Oberfläche als auch in die Tiefe des Einsatzes. Zusammen mit der Emissivität nahe 1 ermöglichen die Stoffeigenschaften des Plexiglases die Messung von örtlich aufgelösten Temperaturverteilungen mit einem optimierten Signal-Rausch-Verhältnis. Die zeitliche Entwicklung der Temperaturverteilung wurde von der Infrarotkamera IRCAM EQUUS 32\%kL mit einem $25 \mathrm{~mm}$ Objektiv, einer Aufnahmerate von $105 \mathrm{~Hz}$ und einer Belichtungszeit von $100 \mu$ s aufgezeichnet. Die Kamera basiert auf einem $640 \times 512$ Pixel FPA-Sensor mit einem sensitiven Spektralbereich von $8.0 \mu \mathrm{m}-9.4 \mu \mathrm{m}$. Der optische Zu- 
gang zur Oberfläche des Modells erfolgt durch ein Germaniumfenster in der Wand des Windkanals. Eine Skizze des Aufbaus ist in Abbildung 3.7 dargestellt. Das Germaniumfenster hat einen Durchmesser von $115 \mathrm{~mm}$ und kann an zwei Positionen montiert werden, die in Strömungrichtung um $150 \mathrm{~mm}$ verschoben sind. Durch die zwei Montagemöglichkeiten in der Windkanalwand können überlappende Messbereiche aufgenommen werden.

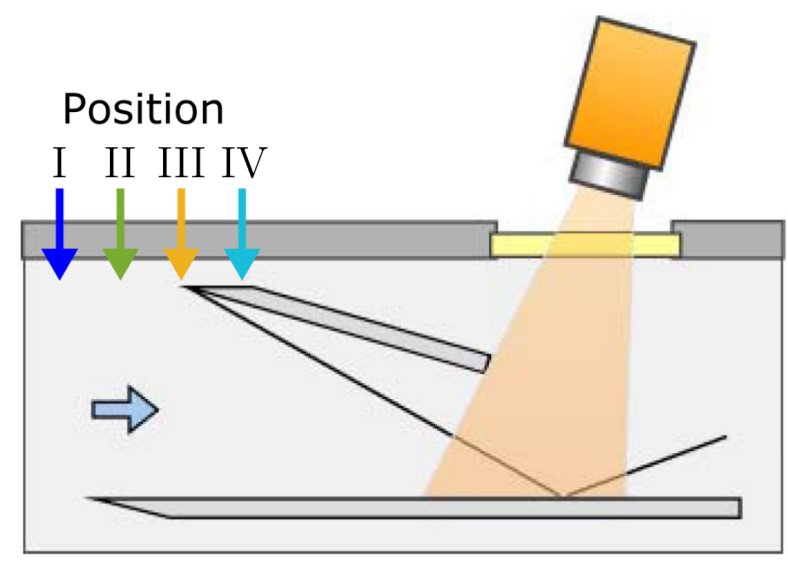

Abbildung 3.7: Skizze der QIRT Messung (angepasst aus (Schülein 2014)).

Die zeitliche Entwicklung der Temperaturverteilung wird mit der DLR-internen Software HeatFit (Schülein 2006, 2014) ausgewertet. Für die Berechnung der Wärmestromdichte $\dot{q}_{\mathrm{w}}\left(t_{n}\right)$ zum Zeitpunkt $t_{n}$ wird das numerische Integrationsverfahren von Cook und Feldermann (Schultz \& Jones 1973, Gl. 83) verwendet:

$$
\dot{q}_{\mathrm{w}}\left(t_{n}\right)=2 \sqrt{\frac{\rho_{\text {Plexi }} c_{p, \text { Plexi }} \lambda_{\text {Plexi }}}{\pi}}\left[\sum_{i=1}^{n} \frac{T_{i}-T_{i-1}}{\sqrt{t_{n}-t_{i}}+\sqrt{t_{n}-t_{i-1}}}\right]
$$

Hierbei steht $T_{i}$ für die Temperatur zum Zeitpunkt $t_{i}$ bei der $i$-ten Aufnahme der Kamera. Die Berechnungsmethode ermöglicht es, zeitlich variierende Wärmestromdichten zu berechnen, wodurch auch Messdaten, die durch die Startphase der Lavaldüse beeinflusst wurden, ausgewertet werden können. Es wird allerdings eine halbunendliche Materialtiefe vorausgesetzt. Diese Annahme ist durch die geringe Wärmeleitfähigkeit des Plexiglaseinsatzes (siehe Tabelle 3.4) in Verbindung mit der begrenzten Messzeit am RWG erfüllt. Die berechneten Wärmestromdichten werden über

$$
S t=\frac{\dot{q}_{\mathrm{w}}}{\rho_{\infty} u_{\infty} c_{p}\left(T_{\mathrm{aw}}-T_{\mathrm{w}}\right)}
$$

in die dimensionslose Stanton-Zahl umgerechnet. Zur Bestimmung von $T_{\mathrm{aw}}$ (vgl. Gl.2.9) wird der Rückgewinnfaktor $r$ benötigt, der allerdings vom Zustand der Grenzschicht abhängt. Für laminare und turbulente Grenzschichten ist der Wert des Rückgewinnfaktors bekannt (Van Driest 1956), jedoch nicht für transitionelle Grenzschichten. Für einen einheitlichen Vergleich der gemessenen Stanton-Zahlen, werden alle gemessenen Fälle 
unabhängig vom realen Zustand der Grenzschicht mit dem Rückgewinnfaktor einer laminaren Grenzschicht $r_{\text {lami }}=\sqrt{P r}=0.85$ skaliert. Bei den gegebenen Testbedingungen $\left(M a=5.98, T_{\infty}=66.6 \mathrm{~K}, T_{\mathrm{w}}=300 \mathrm{~K}, r_{\text {turb }}=0.89\right)$ führt diese Vereinheitlichung zu einer künstlichen Erhöhung der Stanton-Zahl in turbulenten Strömungen um den Faktor

$$
\frac{T_{\mathrm{aw}, \text { turb }}-T_{\mathrm{w}}}{T_{\mathrm{aw}, \text { lami }}-T_{\mathrm{w}}} \approx 1.11
$$

Um die experimentellen Daten mit der theoretischen Kurve nach Van Driest II (vgl. Kapitel 2.1.2 zu vergleichen, wird die Stanton-Zahl für die turbulente Grenzschicht konsequenterweise auch um den Faktor 1.11 erhöht.

Das an der Infrarotkamera eingesetzte Weitwinkelobjektiv verursacht im aufgezeichneten Temperaturbild signifikante Verzerrungen. Durch die Referenzpunkte in der Basisplatte (vgl. Kapitel 3.2), kann die genaue Position des Einsatzes auf den aufgenommenen Bildern identifiziert werden. Das Programm HeatFit erstellt eine markerbasierte 3D-Rekonstruktion der Temperaturbilder und führt eine Rückprojektion der Temperaturverteilung auf die Oberfläche des zugrundeliegenden CAD-Modells durch. Dies liefert für jeden Pixel eine Zuordnung zu den realen Modellkoordinaten. Ein Beispiel für die Auswertung mit HeatFit zeigt Abbildung 3.8. Weitere Details zu HeatFit und der Auswertung der Temperaturverteilungen sind in Schülein (2006) angegeben.

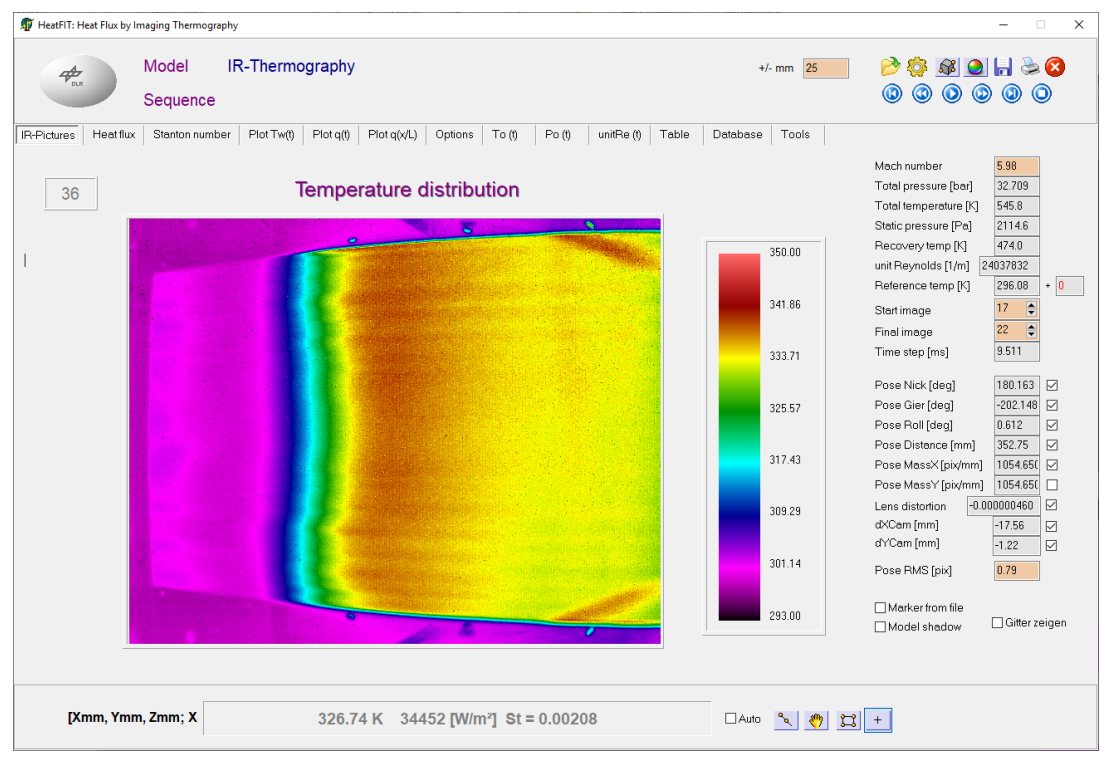

Abbildung 3.8: Beispiel für die gemessene Temperaturverteilung mit SGWW und die Bedienoberfläche von dem Programm HeatFit

Mit den Spezifikationen der Infrarotkamera (Belichtungszeit und Aufnahmerate) können nur niederfrequente Temperaturänderungen erfasst werden. Neben den zeitlichen Einschränkungen ist auch die Sensitivität der Kamera unzureichend, um einzelne turbulente Flecken zu detektieren. Stattdessen werden die zeitlich gemittelten Auswirkungen der Turbulenzflecken auf die Oberflächentemperatur von der Kamera aufgenommen. Im 
Zuge des Transitionsprozesses liefert die QIRT-Technik einen stetigen Übergang zwischen dem St-Niveau der laminaren und turbulenten Grenzschicht, der über die Häufigkeit und Größe der Turbulenzflecken geregelt ist (vgl. Kapitel 2.1.3).

\subsection{Zeitaufgelöste Messungen}

Die eingesetzten Messverfahren zur Untersuchung der Druck- bzw. Dichteschwankungen (PCB und FLDI) zeichnen sich gegenüber den bildgebenden Verfahren durch ihre obere Grenzfrequenz von $1 \mathrm{MHz}$ bzw. $10 \mathrm{MHz}$ aus. Aufgrund dieser Grenzfrequenz können die Messtechniken auch die instationären Vorgänge innerhalb der transitionellen Grenzschicht zeitlich auflösen. Neben der Untersuchung der Zeitsignale, sind auch spektrale Analysen für die Beschreibung der transitionellen Grenzschicht von großem Interesse. Zwei häufig verwendete Methoden sind die Analyse des Leistungsdichtespektrums (LDS) und die Wavelet-Transformation. Beide Methoden können unabhängig von der physikalischen Messgröße angewendet werden, weswegen mit der Vorstellung der Methoden begonnen wird. Anschließend werden die jeweiligen Messtechniken in den entsprechenden Unterkapiteln besprochen. Da die FLDI erstmalig am RWG eingesetzt wurde und die Montage der PCB-Sensoren für die Untersuchung der transitionellen Grenzschicht angepasst werden musste, erfolgt zu beiden Techniken eine detaillierte Analyse der Messeigenschaften.

Spektrale Leistungsdichte Bei den Turbulenzflecken innerhalb der Grenzschicht handelt es sich um eine stochastische Abfolge von Einzelevents. Als Konsequenz ist eine spektrale Untersuchung eines diesbezüglichen Messsignals mittels der diskreten FourierTransformation (DFT) ungeeignet, da die DFT das Spektrum aus einem periodisch fortgesetztem Messsignal berechnet (Meyer 2017). Für die Analyse von zufälligen Schwingungen müssen stattdessen statistische Methoden eingesetzt werden, wie die Abschätzung des Leistungsdichtespektrums (LDS) nach Welch (1967). Hierbei wird das Messsignal in überlappende Segmente unterteilt und mit einer Fensterfunktion gewichtet. Mittels DFT wird das Frequenzspektrum jedes Segments bestimmt und eine Mittelung der Amplitudenquadrate über alle Segmente liefert eine statistische Abschätzung des LDS. Details zur Berechnung des LDS sind in Solomon (1991) beschrieben.

Wavelet-Transformation Die Wavelet-Analyse erweitert die Möglichkeiten der spektralen Auswertung, indem die Schwingungsmoden eines Signals berechnet werden und deren zeitliche Entwicklung in einem Zeit-Frequenz-Diagramm dargestellt wird. Dies ist besonders von Interesse, wenn das Messsignal nicht aus kontinuierlichen Schwingungen besteht, sondern aus separaten Wellenpaketen wie bei der Turbulenzentstehung innerhalb der transitionellen Grenzschicht.

Details über die Implementierung der Wavelet-Transformation sind in Torrence \& Compo (1998) zu finden. Im Folgenden werden die notwendigen Schritte für das Verständnis der Wavelet-Transformation kurz erläutert. Die Grundlage der WaveletTransformation besteht in der Wahl eines Mutter-Wavelets $\Psi_{0}$. Dabei sollte die Form 
des Mutter-Wavelets möglichst ähnlich zu den vermuteten Strukturen im Zeitsignal sein, da die Waveletamplitude durch eine Faltung berechnet wird. In dieser Arbeit wurde das Morlet-Wavelet (Torrence \& Compo 1998) gewählt, da es dem Wellenpaket einer instabilen Mode ähnelt (Weisberger et al. 2019):

$$
\Psi_{0}(\eta)=\pi^{-1 / 4} \mathrm{e}^{i \omega_{0} \eta} \mathrm{e}^{-\eta^{2} / 2}
$$

Hierbei stellt $\eta$ eine entdimensionalisierte Zeit dar und $\omega_{0}$ eine dimensionslose Wellenzahl. Mit $\omega_{0}$ kann das Verhältnis zwischen Frequenz- und Zeitauflösung der WaveletTransformation eingestellt werden. In Abbildung 3.9 ist das Mutter-Wavelet mit der gewählten Wellenzahl $\omega_{0}=4 \pi$ dargestellt.

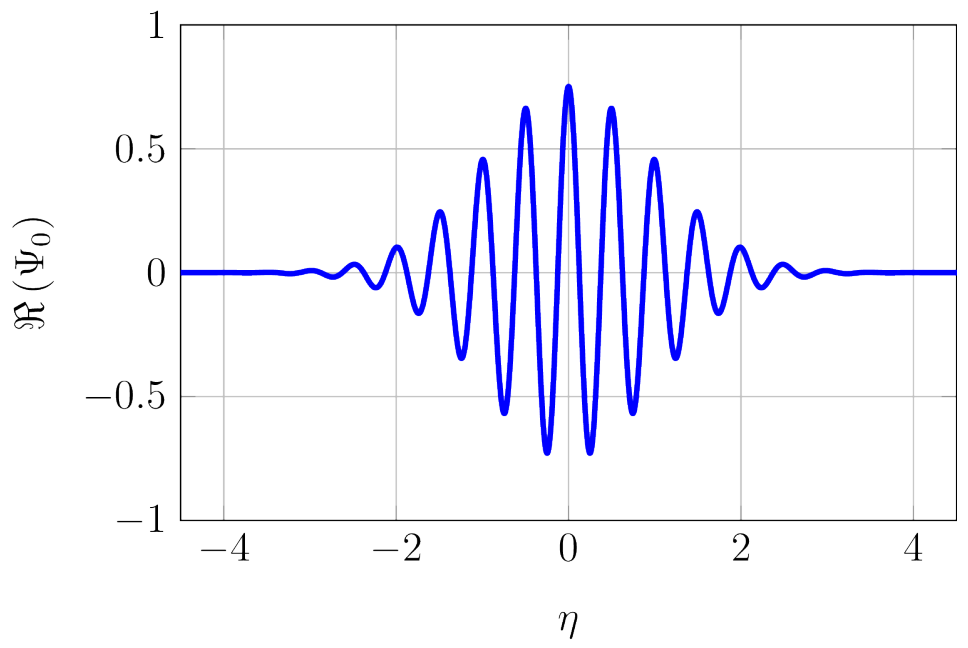

Abbildung 3.9: Realteil des gewählten Mutter-Wavelet mit $\omega_{0}=4 \pi$

Die Berechnung der kontinuierlichen Wavelet-Transformation besteht nun in der Faltung des Messsignals $x(t)$ mit skalierten und verschobenen Varianten der Mutter-Wavelet (Mertins 2013):

$$
W(s, b)=\frac{1}{\sqrt{s}} \int_{-\infty}^{\infty} x(t) \Psi^{*}\left(\frac{t-b}{s}\right) \mathrm{d} t
$$

Bei $\Psi^{*} / \sqrt{s}$ handelt es sich um einen komplex konjugierten Repräsentanten des MutterWavelets $\Psi_{0}$ mit einer einheitlich skalierten Energie. Der Parameter $b$ verursacht eine zeitliche Translation des Wavelets. Mittels $s$ wird eine zeitliche Streckung bzw. Stauchung des Wavelets durchgeführt, um verschiedene Frequenzen zu untersuchen. Für das MorletWavelet kann der Skalierungsparameter $s$ in eine Frequenz mittels

$$
f=\frac{\omega_{0}+\sqrt{2+\omega_{0}^{2}}}{4 \pi s}
$$

umgerechnet werden. Durch Variation von $b$ und $s$ kann eine Heatmap erstellt werden, die die zeitliche Entwicklung der Waveletamplitude bei diversen Frequenzen zeigt. Die 
Berechnung der Waveletamplitude anhand einer Faltung bedeutet allerdings, dass die Waveletamplitude nicht die reale Amplitude der Schwingung wiedergibt.

Da das Wavelet zur Bestimmung von niedrigen Frequenzen gestreckt wird, werden bei der Faltung mehr Messpunkte berücksichtigt, wodurch die Frequenz feiner bestimmt wird. Allerdings verschlechtert sich die zeitliche Lokalisierung der Schwingung. Bei einer Stauchung des Wavelets verhalten sich die Eigenschaften entgegengesetzt. Diese Adaptivität bezüglich der Zeit-Frequenz-Bestimmung ist eine positive Eigenschaft der WaveletTransformation, da die Dynamik hochfrequenter Ereignisse wiedergeben und gleichzeitig die Frequenz lang andauernder Schwingungen genau bestimmt wird.

\subsubsection{Druckmessungen}

Für die Druckmessungen wurden piezoelektrische Sensoren $P C B$-132A31 der Firma $P C B$ Synotech $\mathrm{GmbH}$ eingesetzt. Diese Sensoren - im Folgenden auch als PCB-Sensoren bezeichnet - können Druckschwankungen zwischen $11 \mathrm{kHz}-1 \mathrm{MHz}$ messen. Weitere Details können dem entsprechenden Datenblatt im Anhang A.2 entnommen werden. Für die Montage der 16 verwendeten PCB-Sensoren wurde ein separater Platteneinsatz (vgl. Abb. 3.10a gefertigt. Die Sensoren sind diagonal angeordnet, um Nachlaufeffekte zwischen den Messpositionen zu unterbinden. Der Einsatz selbst kann in zwei Ausrichtungen in die Basisplatte eingebaut werden, wodurch die Druckschwankungen der Strömung sowohl stromauf als auch stromab des Einsatzmittelpunktes messbar sind. Da der Einsatz dafür gedreht werden muss, ändern sich die Messpositionen punktsymmetrisch. Die Koordinaten der PCB-Sensoren sind für beide Ausrichtungen in Tabelle A.1 aufgelistet. Für die Datenerfassung wurden die A/D-Wandlermodule TRION-1620-ACC-6-L1B der DEWETRON GmbH verwendet. Während der Messung wurde das Messsignal der 16 PCB-Sensoren mit $2 \mathrm{MHz}$ abgetastet. Die Abtastrate ist unter Berücksichtigung der Nyquist-Frequenz stimmig mit der vom Hersteller angegeben Grenzfrequenz der PCBSensoren.



(a) Einsatzplatte mit der Lage der PCB-Sensoren

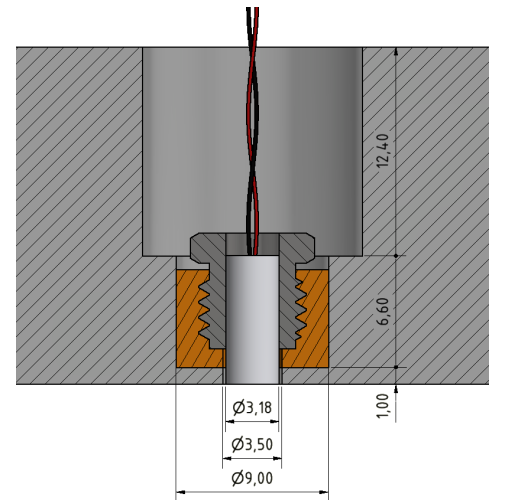

(b) Einbau der PCB-Sensoren in die Platte. Das eingegossene Silikon ist orange dargestellt

Abbildung 3.10: Zeichnungen zum Einbau der PCB-Sensoren 
Die vom Hersteller empfohlene Methode zum Einbau der PCB-Sensoren (vgl. Abb. A.1) sieht vor, die Sensoren in eine Befestigungsmutter einzukleben und die Mutter über ein Außengewinde in das Modell zu verschrauben. Vortests zeigten allerdings, dass sich bei der Messung mechanische Schwingungen vom Modell auf die Sensoren übertragen und das Messsignal verfälschen. Der Einfluss dieser Störungen überlagerte die Druckschwankungen der Strömung und machte eine strömungsmechanische Analyse der Messdaten unmöglich. Von Problemen bezüglich mechanischen Schwingungen wird auch in den Arbeiten von Willems et al. (2015), Weisberger et al. (2019) berichtet und Schneider (2019), Ort \& Dosch (2019) untersuchten den Einfluss unterschiedlicher Einbauvarianten auf die Schwingungsübertragung. In der aktuellen Arbeit konnte die Schwingungsübertragung reduziert werden, indem der Sensor samt Befestigungsmutter in Silikon eingegossen wurde. Nach dem Aushärten wurde sowohl der Sensor aus der Silikonhülle als auch die Silikonhülle aus der Einsatzplatte gezogen, um die Verbindung zwischen den Komponenten einmalig aufzutrennen. Anschließend wurde der Sensor mit der Silikonhülle wieder in die Einsatzplatte gesteckt. Der Einbau der PCB-Sensoren ist in Abbildung 3.10b dargestellt. Um eine direkte Schwingungsübertragung auf den Sensor zu verhindern, darf der Sensor die Einsatzplatte nicht berühren. Aus diesem Grund existiert um den Sensor ein radialer Spalt von $160 \mu \mathrm{m}$, der zwar zusätzliche Störungen in der Grenzschicht verursacht, aber bewusst nicht mit Silikon aufgefüllt wurde, weil nach den gewonnenen Erfahrungswerten solch dünne Silikonschichten keine ausreichende Dämpfung der Schwingungsübertragung gewährleisten.

Für eine Verifizierung der reduzierten Schwingungsübertragung gegenüber der Herstellervariante wurden zwei Sensoren auf beide Arten in eine Testplatte eingebaut. Mittels eines Hammerschlagtests, hierbei wird mit einem harten Gegenstand auf die Platte geschlagen, werden die Schwingungsmoden der Testplatte angeregt und deren Übertragung auf die Sensoren kann untersucht werden. Eine gleichzeitige Messung des Schlagimpulses für eine anschließende Normierung war nicht notwendig, da bei diesem Vergleich beide Testsensoren den identischen mechanischen Schwingungen ausgesetzt waren. Um ein repräsentatives Ergebnis für die Schwingungsübertragung zu erhalten und die Variationen beim Schlagimpuls etwas auszugleichen, wurden die LDS aus 80 Anschlägen gemittelt (Abb. 3.11). Beim Hammerschlagtest wurden neben den mechanischen Schwingungen innerhalb der Testplatte auch akustische Schwingungen erzeugt, die von einem dritten Sensor aufgezeichnet wurden, der nicht in der Testplatte verbaut war. Neben den akustischen Schwingungen zeigt der dritte Sensor auch das Grundrauschen und verkörpert den idealen Kurvenverlauf ohne Schwingungsübertragung. Die Einbauvariante des Herstellers liegt signifikant über der Referenzkurve des Grundrauschens und verdeutlicht, wie gravierend das Messsignal durch mechanische Vibrationen beeinflusst wird.

Durch die Silikonhülle konnten die Schwingungen in dem Bereich $f \approx 11 \mathrm{kHz}-180 \mathrm{kHz}$ signifikant gedämpft werden. Für $f>180 \mathrm{kHz}$ hat sich das Antwortverhalten allerdings verschlechtert. Neben der eingeschränkten Verbesserung führt die Kavität um jeden Sensor zu einer zusätzlichen Beeinflussung der Grenzschicht, sodass für zukünftige Messkampagnen an besseren Maßnahmen zur Schwingungsentkopplung gearbeitet werden sollte.

Das Eingießen der Sensoren und das anschließende erneute Zusammenstecken in die elastische Silikonhülle führt zu Abweichungen in der Ausrichtung der Sensoren. Hiervon 


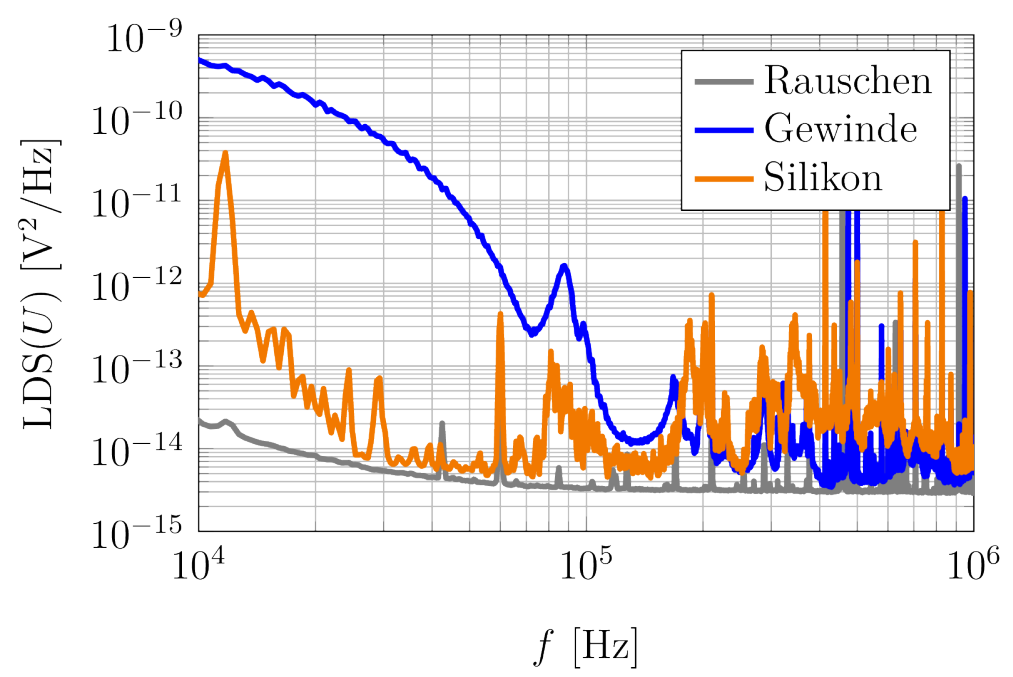

Abbildung 3.11: Schwingungsübertragung der PCB-Sensoren für unterschiedliche Einbauvarianten

ist sowohl die Sensorachse relativ zur Wandnormalen, der bündige Übergang zwischen Sensorfläche und Wand als auch die Zentrierung im Loch betroffen, wobei letztere bereits an der fehlenden Zentrierung des eigentlichen Messelementes innerhalb des Sensors selbst scheitert (Schneider 2019). Obwohl auf die Ausrichtung der Sensoren beim Einsetzen geachtet wurde, führen die Abweichungen zwischen den Sensoren zu unterschiedlichen Antwortverhalten bezüglich der mechanischen Schwingungen. Aus diesem Grund wurden die Sensoren im eingebauten Zustand erneut bezüglich der Schwingungsübertragung getestet. Ein Beispiel ist in Abbildung 3.12a gezeigt.

Das Messsignal der PCB-Sensoren kann mit dem sensorspezifischen Kalibrierungsfaktor (vgl. Tabelle A.1) in einen Druck umgerechnet werden und setzt sich aus drei Komponenten zusammen: dem Grundrauschen, den mechanischen Schwingungen und den Druckschwankungen innerhalb der Grenzschicht. Ist das Messsignal aufgrund der mechanischen Schwingungen mindestens vergleichbar zu den strömungsmechanischen Druckschwankungen, liegt eine relevante Verfälschung des Messsignals vor. Da vor der Messung nicht klar ist, welcher Effekt bei der jeweiligen Frequenz überwiegt, ergibt sich die Gefahr aus den Messdaten falsche Schlussfolgerungen zu ziehen. Die Problematik ist am Beispiel einer laminaren Grenzschichtmessung in Abb. 3.12b verdeutlicht. Analysiert man das laminare LDS unter Zuhilfenahme von Abb. 3.12a, so kann man schlussfolgern, dass die Windkanaldaten für $f>180 \mathrm{kHz}$ durch mechanische Vibrationen dominiert sind. Insbesondere die charakteristischen Peaks bei $f \approx 195 \mathrm{kHz}$ oder $f \approx 345 \mathrm{kHz}$ lassen sich in beiden Abbildungen wiedererkennen. Eine Auswertung des Leistungsdichtespektrums zu unterschiedlichen Zeitpunkten während der Messzeit zeigte eine gleichbleibende Amplitude der mechanischen Schwingungen. Da die mechanischen Schwingungen über die Messzeit nicht abklingen, obgleich die Schwingungen beim Hammerschlagtest bereits nach wenigen Millisekunden vollständig abgeklungen waren, werden die mechanischen Schwingungen im Windkanal vermutlich kontinuierlich angeregt. 




(a) Spektrum bei Schwingungsanregung des Einsatzes durch Anschlagen

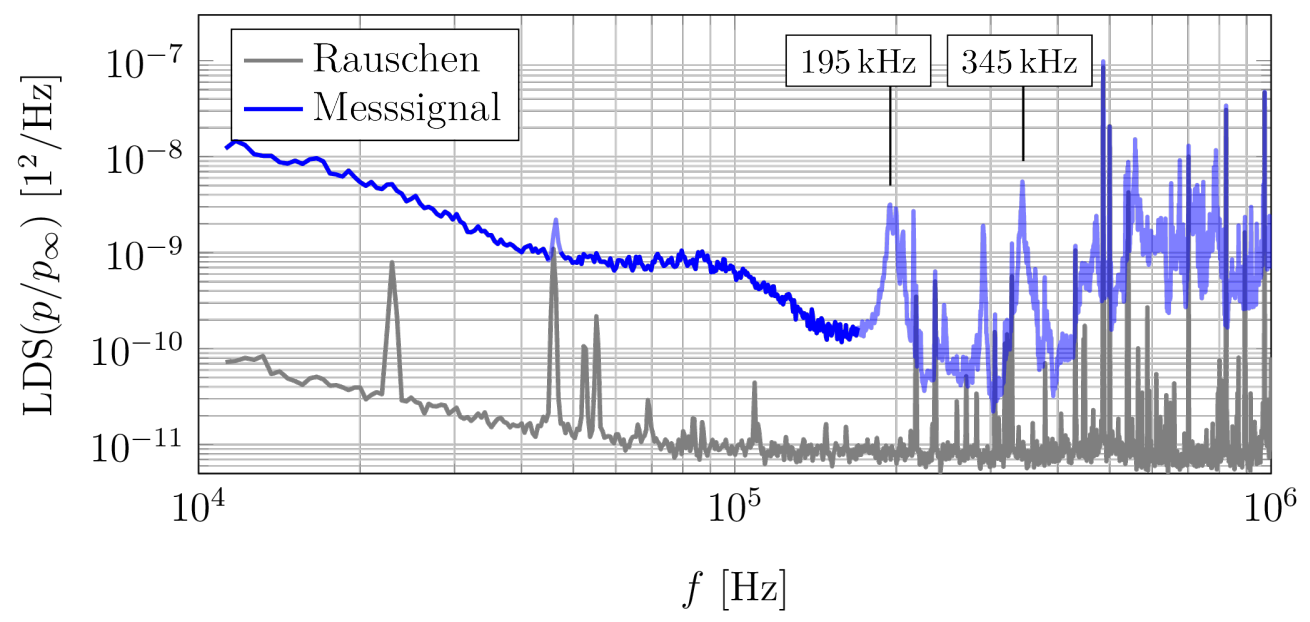

(b) Spektrum bei einer laminaren Grenzschicht

Abbildung 3.12: LDS eines PCB-Sensors

Der Peak bei $f=87 \mathrm{kHz}$ kann allerdings nicht in den Windkanaldaten (Abb. 3.12b) beobachtet werden. Hierbei ist unklar ob bzw. mit welcher Amplitude diese spezifische mechanische Schwingung im Experiment angeregt wird. Der Vergleich der beiden Spektren zeigt, dass in diesem Fall zumindest der Frequenzbereich bis $f \approx 180 \mathrm{kHz}$ für die Untersuchungen der Grenzschichtinstabilitäten genutzt werden kann.

Um auch bei den anderen Sensoren und Messbedingungen zu beurteilen, welche Frequenzbereiche durch die mechanischen Schwingungen verfälscht sind, wird folgendes Schema verwendet. Als erster Schritt werden mehrere LDS des selben Sensors bei unterschiedlichen Strömungsbedingungen untersucht. Sind Frequenzbereiche erkennbar, die nicht zum restlichen Spektrum passen und in mehreren Spektren auffindbar sind, so ist dieser Frequenzbereich vermutlich durch mechanische Schwingungen beeinflusst. Die sichtbare Unregelmäßigkeit in den LDS gilt als notwendiges Kriterium. Im zweiten 
Schritt wird überprüft ob der verdächtige Frequenzbereich auch beim Hammerschlagtest (vgl. Abbildung 3.12a) auffällig ist. Dies gilt als hinreichende Bedingung und falls beide Bedingungen erfüllt sind, wird der besagte Frequenzbereich in dem LDS halbtransparent dargestellt (vgl. Abb. 3.12b). Diese visuelle Einstufung ermöglicht eine vollständige Darstellung des gemessenen Spektrums und gleichzeitig wird die Trennung zwischen Störsignalen und strömungsmechanischen Effekten erleichtert.

\subsubsection{Fokussierte Laser-Differentialinterferometrie}

Smeets \& George beschrieben bereits 1973 die Anwendung der fokussierten LaserDifferentialinterferometrie (FLDI) für Dichtemessungen in Windkanälen. Aufgrund der damals limitierten Möglichkeiten bezüglich der Photodiode, Datenerfassung und Qualität der Laser wurde die Messtechnik kaum genutzt. Erst seit Parziale et al. (2012) die Anwendung der FLDI-Technik demonstrierte, finden vermehrt Messungen mit FLDI statt, um die Qualität der freien Anströmung in Windkanälen zu untersuchen (Parziale et al. 2012, 2014, Settles \& Fulghum 2016, Birch et al. 2020) oder um die Instabilitäten innerhalb der Grenzschicht (Parziale et al. 2013, Parziale 2013, Weisberger et al. 2019) zu messen. Theoretische Betrachtungen zu dem Zusammenhang zwischen Messsignal und Dichteschwankungen wurden von Fulghum (2014), Schmidt \& Shepherd (2015), Lawson et al. (2020) durchgeführt. Mit einem Doppelfokus-FLDI Aufbau (Jewell et al. 2019, Weisberger et al. 2019) werden einzelne turbulente Spots innerhalb der Grenzschicht verfolgt und deren Phasengeschwindigkeit ermittelt.

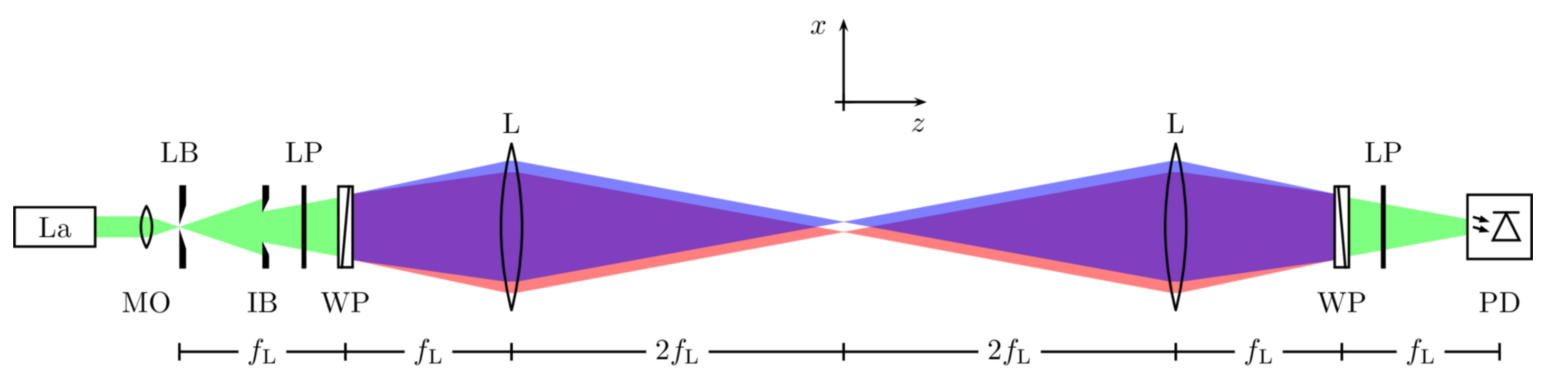

Abbildung 3.13: Skizze des FLDI Aufbaus. Der aufgeteilte Strahl ist blau und rot dargestellt. Überlappende Bereiche sind violett

Die FLDI Aufbauten in den Arbeiten von Parziale (2013), Schmidt \& Shepherd (2015), Lawson et al. (2020) dienten als Vorlage für den am RWG verwendeten Aufbau. Dieser ist in Abbildung 3.13 gezeigt und Tabelle 3.5 listet die verwendeten Komponenten. Als Laser wurde ein $O B I S 660 L X$ von Coherent mit einer Wellenlänge von $\lambda_{\mathrm{OBIS}}=660 \mathrm{~nm}$ verwendet. Dem Strahlengang folgend wurde der Laserstrahl zunächst mit einem Mikroskopobjektiv fokussiert. Im Brennpunkt befand sich eine Lochblende mit einem Öffnungsdurchmesser von $50 \mu \mathrm{m}$, um einen definierten Fokuspunkt zu erzeugen. Nach der Lochblende weitete sich der Laserstrahl wieder auf und eine zusätzliche Irisblende reduzierte den Durchmesser des Laserstrahls auf die freie Apertur des nachfolgenden WollastonPrismas. Zwischen der Irisblende und dem Wollaston-Prisma befand sich ein linerarer 
Polarisationsfilter. Die Polarisationsrichtung des Lichtes wurde so gewählt, dass sie um $45^{\circ}$ verdreht war zur optischen Achse des nachfolgenden Prismas. Das Wollaston-Prisma teilte den Laserstrahl unter einem Winkel von $\varphi_{\mathrm{WP}}=2^{\prime}$ in zwei orthogonal polarisierte Teilstrahlen auf. Eine Sammellinse mit einer Brennweite von $f_{\mathrm{L}}=300 \mathrm{~mm}$ fokussierte die beiden Strahlen. Da das Prisma in der Fokusebene der Sammellinse positioniert wurde, verliefen die Achsen beider Teilstrahlen hinter der Linse parallel und in einem räumlichen Abstand von $\Delta x=2 \tan \left(\varphi_{\mathrm{WP}} / 2\right) f_{\mathrm{L}}=175 \mu \mathrm{m}$. Aufgrund dieses Abstandes überlappten sich die Querschnitte der beiden Lichtkegel fast vollständig. Lediglich in der Nähe der Fokusebene waren die beiden Strahlen komplett voneinander separiert. In diesem Bereich lag die maximale Empfindlichkeit der FLDI Technik bezüglich einer optischen Phasenverschiebung. Außerhalb der Fokusebene war die Sensitivität aufgrund der Überlappung und der größeren Strahldurchmesser gedämpft. Nach der Fokusebene wurden die divergenten Teilstrahlen durch eine zweite Sammellinse erneut gebündelt. Ein zweites Wollaston-Prisma rekombinierte die Teilstrahlen und ein weiterer linearer Polarisationsfilter erzeugte eine einheitliche Polarisationsrichtung. Unterschiede in der Phasenbeziehung beider Teilstrahlen wurden als Intensitätsänderungen aufgrund von Interferenz mittels einer Photodiode detektiert. Wie in Ramprakash et al. (2019) erklärt, wurde durch die Verschiebung des zweiten Wollaston-Prismas quer zur optischen Achse die Phasenbeziehung zwischen den Teilstrahlen so eingestellt, dass der FLDI-Aufbau die maximale Empfindlichkeit bezüglich Dichtestörungen besaß. Das Messsignal der Photodiode wurde von einem DPO 7254 Oszilloskops von Tektronix mit einer Abtastrate von $25 \mathrm{MHz}$ im Hi-Res Modus aufgezeichnet. In diesem Modus wurde das Messsignal mit der maximalen Abtastrate des Analog-Digital-Wandlers aufgenommen, intern gemittelt und mit der eingestellten Abtastrate abgespeichert, was zu einer Rauschreduzierung und effektiven Steigerung der Bit-Tiefe führte.

\begin{tabular}{ll}
\hline Kürzel & Beschreibung \\
\hline La & Dauerstrichlaser OBIS 660LX, $\lambda_{\text {OBIS }}=660 \mathrm{~nm}, P=100 \mathrm{~mW}$ \\
MO & Mikroskopobjektiv mit 20-facher Vergrößerung \\
LB & Lochblende, $50 \mu \mathrm{m}$ Durchmesser \\
IB & Irisblende \\
LP & Linearer Polarisationsfilter \\
WP & 1" Wollaston-Prisma, Aufspaltungswinkel $\varphi_{\mathrm{WP}}=2^{\prime}$ \\
L & 2" Sammellinse, $f_{\mathrm{L}}=300 \mathrm{~mm}$ \\
PD & Photodiode, Thorlabs DET36A2 \\
\hline
\end{tabular}

Tabelle 3.5: Komponenten des verwendeten FLDI Aufbaus

Aufgrund des optischen Aufbaus handelt es sich bei FLDI um eine nicht-invasive Messtechnik, die aufgrund der Fokussierung lokale Messungen im Bereich der Fokusebene ermöglicht. Da die Sensitivität außerhalb der Fokusebene abnimmt, können bei FLDI die großskaligen Dichteänderungen in der Grenzschicht der Windkanalwand ausgeblendet werden. Im Folgenden werden alle Komponenten vor der Fokusebene als Sendeseite 
und alle Komponenten nach der Fokusebene als Empfängerseite des FLDI Aufbaus bezeichnet.

In bisherigen Windkanalmessungen wurde die FLDI-Messtechnik hauptsächlich an rotationssymmetrischen Modellen oder in der freien Anströmung eingesetzt. Bei der Verwendung der FLDI-Messtechnik zur Untersuchung der Grenzschicht an einer ebenen Platte entsteht ein Problem bezüglich der Abschattung des Strahlengangs wie es Abbildung 3.14 verdeutlicht. Bei der Standardversion des FLDI-Aufbaus wird ein Teil des defokussierten Lichtstrahls blockiert beziehungsweise hinter der Fokusebene am Modell reflektiert. Um dieses Problem zu lösen, ersetzten Houpt \& Leonov (2018) die Sammellinsen durch Zylinderlinsen (CFLDI). Durch die Modifizierung des Strahlengangs, entsteht ein paralleler Lichtschnitt, der oberhalb der ebenen Geometrie fokussiert wird. Houpt \& Leonov (2019) zeigten, dass auch mit dem abgewandelten Aufbau die Detektion von Störungen innerhalb von Grenzschichten möglich ist, allerdings vergrößert sich im Vergleich zum klassischen FLDI das sensitive Gebiet entlang der optischen Achse.

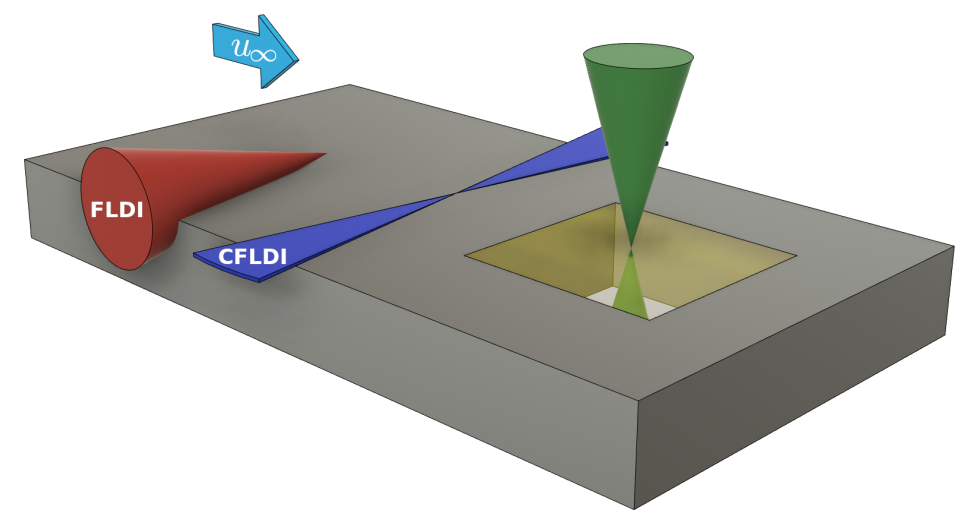

Abbildung 3.14: Skizze vom Strahlengang beim klassischen FLDI-Aufbau und bei der Verwendung von Zylinderlinsen (CFLDI). Beim klassischen FLDI wird an ebenen Geometrien ein Großteil des Strahldurchmessers abgeschattet. Um der Abschattung zu entgehen, kann ein Glas in die Platte eingebaut werden, wobei der Strahlengang senkrecht zur Oberfläche verläuft.

In dieser Arbeit wird ein anderer Ansatz verfolgt. Für die Basisplatte wurde ein neuer Einsatz angefertigt, in dem ein Glasfenster eingelassen wurde (vgl. Abbildung 3.14 und 3.15). Das Glasfenster ermöglicht es den Strahlengang durch die ebene Platte verlaufen zu lassen. Dadurch kann die Fokusebene der FLDI-Technik in die Grenzschicht der ebenen Platte gelegt werden, ohne eine Abschattung des Strahlengangs zu erhalten. Zusätzlich wurde ein $46 \times 270 \mathrm{~mm}^{2}$ Schacht von der Windkanalwand bis zur Unterseite des Glaseinsatzes geführt. Ein Schnitt dieses Aufbaus ist in Abbildung 3.15 gezeigt. Durch den Schacht wird eine Interaktion der Laserstrahlen mit der Strömung unterhalb der Basisplatte vollständig unterbunden, sodass die Phasenverschiebungen zwischen den Teilstrahlen nur durch Effekte auf der Oberseite der ebenen Platte entstehen können.

Die FLDI Technik wurde im Rahmen dieser Arbeit zum ersten Mal am RWG eingesetzt, wodurch Erfahrungswerte bezüglich der Größe des sensitiven Volumens oder dem 




Abbildung 3.15: Schnitt durch das Plattenmodell samt Schacht. Der Schacht beginnt an der Unterseite der ebenen Platte und endet an der Wand des Windkanals. Durch den Schacht wird der Strahlenverlauf des FLDI-Aufbaus vollständig von der Strömung unterhalb der ebenen Platte isoliert.

Antwortverhalten der Messtechnik auf Dichtestörungen fehlen. Um diese offenen Punkte zu klären, wurden neben den Windkanalmessungen zusätzliche Untersuchungen des FLDI-Aufbaus ohne Strömung durchgeführt.

\section{Laserprofil}

Zur Überprüfung des Strahlengangs wurde mit der Kamera TaperCamD-UCD23 von DataRay das Strahlprofil beider Teilstrahlen an verschiedenen Positionen entlang der optischen Achse gemessen. Die skalierten Intensitätsverteilungen in Abbildung 3.16 zeigen den konvergenten Verlauf der Teilstrahlen bis zur Fokusebene. Die Profilmessungen verdeutlichen wie der Übergang von den überlappenden Teilstrahlen zu zwei separaten Strahlen in der Realität abläuft. Außerhalb der Brennebene weisen die Intensitätsprofile deutliche Beugungseffekte auf und zeigen einen abrupten Intensitätsabfall an den Profilrändern. Die Ursache für beide Effekte liegt in der verwendeten Irisblende. Aufgrund der Abschattung erzeugt die Irisblende einen steilen Intensitätsabfall an den Rändern der Profile. Gleichzeitig sind die Kanten der Irisblende der Ursprung der Beugungseffek-

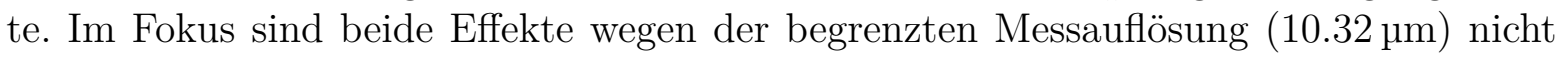
mehr sichtbar. Um die Laserprofile im Fokus quantitativ zu beschreiben, wurden zwei Gaußprofile

$$
I(r)=\exp \left(-\frac{2 r^{2}}{\omega_{\mathrm{G}}^{2}}\right)
$$


durch die skalierte Intensitätsverteilung gefittet, wobei $r$ den radialen Abstand vom jeweiligen Fokusmittelpunkt beschreibt und $\omega_{\mathrm{G}}$ den Strahlradius widerspiegelt. Auf Basis der Regression haben die Fokuspunkte einen Abstand von $\Delta x=178 \mu \mathrm{m}$ und $\omega_{\mathrm{G}}=23 \mu \mathrm{m}$. Beide Werte sind stimmig zu den erwarteten Werten $(\Delta x=175 \mu \mathrm{m}$ und $25 \mu \mathrm{m}$ für den Radius der Lochblende), insbesondere unter Berücksichtigung der unzureichenden Auflösung des Messgerätes im Fokus und der Abweichung des experimentellen Strahlprofils vom idealen Gauß-Strahl.

\section{Relation zwischen Dichtefeld und Messsignal}

Nach der Überprüfung des Strahlenverlaufs folgt eine Analyse des Messsignals. Laut dem Datenblatt der verwendeten Photodiode erzeugt einfallendes Licht einen elektrischen Strom, der proportional zur Intensität $I$ ist und über einen Abschlusswiderstand $R_{\mathrm{A}}$ als Spannung $U$ vom Oszilloskop gemessen wird. Dieser Zusammenhang kann mathematisch durch

$$
U=C R_{\mathrm{A}} I
$$

angegeben werden, wobei $C$ eine Proportionalitätskonstante der Photodiode ist. Die Beziehung zwischen der Lichtintensität und der Phasendifferenz $\Delta \phi$ des Interferometers ist gegeben durch (Demtröder 2009)

$$
I=I_{\mathrm{A}}+I_{\mathrm{B}}+2 \hat{l}_{\mathrm{A}} \cdot \hat{l}_{\mathrm{B}} \sqrt{I_{\mathrm{A}} I_{\mathrm{B}}} \cos (\Delta \phi) .
$$

Die Indizes A und B bezeichnen jeweils einen Teilstrahl und $\hat{l}$ deren Polarisationsrichtung. Beim FLDI-Aufbau besitzen beide Teilstrahlen per Konstruktion die gleiche Intensität $\left(I_{\mathrm{A}}=I_{\mathrm{B}}=I_{0}\right)$, denn die Polarisationsrichtung des Lichtes ist um $45^{\circ}$ zur optischen Achse des ersten Wollaston-Prismas gedreht. Nach dem Passieren des zweiten Polarisationsfilters liegt eine einheitliche Polarisationsrichtung vor, sodass $\hat{l}_{\mathrm{A}} \cdot \hat{l}_{\mathrm{B}}=1$. Durch Justieren der Phasendifferenz zwischen den beiden Teilstrahlen, wurde der FLDI-Aufbau auf seine maximale Empfindlichkeit gestellt. Anhand von Gleichung 3.9 wird klar, dass die maximale Empfindlichkeit bei $\Delta \phi= \pm \pi / 2$ vorliegt. Während der Messung variiert die Phasendifferenz, da Störungen eine Veränderung der optischen Wegdifferenz zwischen den Teilstrahlen $\Delta s_{\text {opt }}$ hervorrufen. Für die Phasendifferenz bedeutet dies

$$
\Delta \phi= \pm \frac{\pi}{2}+\frac{2 \pi \Delta s_{\mathrm{opt}}}{\lambda_{\mathrm{OBIS}}}
$$

Unter Verwendung der Gleichungen 3.8, 3.9 und 3.10 ergibt sich für das gemessene Spannungssignal:

$$
U=2 R_{\mathrm{A}} C I_{0} \cdot\left[1 \pm \sin \left(\frac{2 \pi \Delta s_{\mathrm{opt}}}{\lambda_{\mathrm{OBIS}}}\right)\right] .
$$

Aufgrund der experimentellen Justierung gilt vor Messbeginn $\Delta s_{\text {opt }}=0$ und somit für die Spannung $U_{0}=2 R_{\mathrm{A}} C I_{0}$. Eine Skalierung von Gleichung 3.11 mit der Spannung $U_{0}$ 

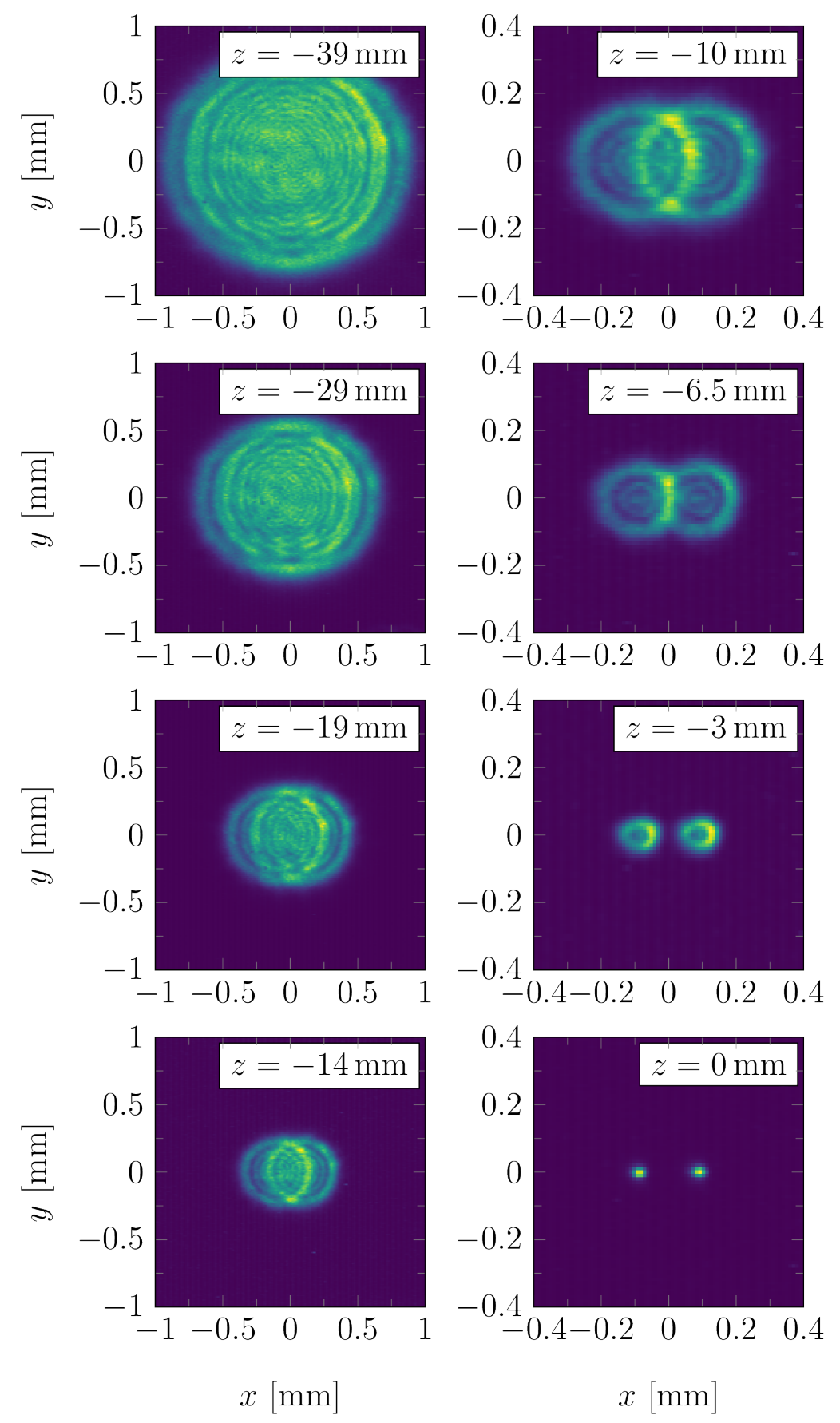

Abbildung 3.16: Skalierte Intensitätsverteilungen der Laserstrahlprofile beim FLDIAufbau an mehreren Positionen entlang der optischen Achse 
ermöglicht die Berechnung der optischen Wegdifferenz aus dem Messsignal:

$$
\Delta s_{\mathrm{opt}}= \pm \frac{\lambda_{\mathrm{OBIS}}}{2 \pi} \arcsin \left(\frac{U}{U_{0}}-1\right)
$$

Im Experiment konnte nicht vermieden werden, dass beim Öffnen des Schiebers Schmieröl von der Strömung mitgerissen wurde, welches sich an den Glasscheiben ablagerte und die Lichtintensität reduzierte. Das Starten des Windkanals reduziert somit den Wert von $U_{0}$, weshalb $U_{0}$ durch die zeitliche Mittelung des Spannungssignals während der Messzeit approximiert wurde. Diese Approximation ist möglich, da $U$ während der Messzeit um den reduzierten Wert $U_{0}$ schwankt.

Falls $\left|\Delta s_{\text {opt }}\right|$ während der Messung größer als $\lambda_{\text {OBIS }} / 4$ wird, ergibt sich aufgrund der Periodizität der Sinusfunktion in Gleichung 3.11 eine Mehrdeutigkeit in der inversen Bestimmung von $\Delta s_{\text {opt }}$ anhand von Gleichung 3.12. Eine Sichtung der Messsignale belegt, dass die extremalen Spannungswerte des Interferometers nie erreicht werden, sodass keine Mehrdeutigkeit bezüglich $\Delta s_{\text {opt }}$ vorliegt.

Die Phasendifferenz des FLDI-Interferometers entsteht durch die optische Wegdifferenz der beiden Teilstrahlen. Der optische Weg wird durch das Integral über den lokalen Brechungsindex entlang der Lichtrichtung berechnet. Da die beiden Teilstrahlen parallel zur optischen Achse verlaufen und um $\Delta x$ zueinander versetzt sind, kann die optische Wegdifferenz durch

$$
\Delta s_{\mathrm{opt}}=s_{\mathrm{opt}, \mathrm{A}}-s_{\mathrm{opt}, \mathrm{B}}=\int\left[n_{\mathrm{A}}(z)-n_{\mathrm{B}}(z)\right] \mathrm{d} z
$$

berechnet werden. Bei dem Brechungsindex $n$ handelt es sich um einen effektiven Berechungsindex, der sich aus der Mittelung über den lokalen Strahldurchmesser ergibt. Mit der Gladstone-Dale-Beziehung (Gladstone \& Dale 1863) $n=\rho K_{\mathrm{GD}}+1$ erfolgt die Umrechnung vom Brechungsindex zur Dichte:

$$
\Delta s_{\mathrm{opt}}=K_{\mathrm{GD}} \int \Delta \rho_{\mathrm{AB}}(z) \mathrm{d} z .
$$

Aufgrund des Integrals in Verbindung mit der effektiven Dichtedifferenz $\Delta \rho_{\mathrm{AB}}(z)$ ist es unmöglich von der gemessenen optischen Wegdifferenz auf die Dichteverteilung zurückzurechnen. Um dennoch zu verstehen, wie die FLDI-Technik auf Dichtefelder reagiert, haben Fulghum (2014) und Schmidt \& Shepherd (2015) Systemfunktionen für akademische Dichtefelder berechnet. Die Systemfunktion $H$ beschreibt für jede Frequenz das Verhältnis der messbaren Signalamplitude zur echten Störungsamplitude. Die Systemfunktion der FLDI-Technik entsteht im wesentlichen durch zwei Effekte. Zum einen muss der endliche Abstand $\Delta x$ der beiden Fokuspunkte berücksichtigt werden und zum anderen die Intensitätsverteilung der Laserprofile entlang der optischen Achse. Birch et al. (2020) führten zwei weitere Systemfunktionen ein, um den Einfluss eines Signalverstärkers bzw. der Grenzfrequenz der Photodiode auf die Frequenzantwort der FLDI-Technik zu beschreiben. Die beiden von Birch et al. (2020) eingeführten Systemfunktionen sind in der aktuellen Arbeit wegen des gewählten Messequipments irrelevant. Im Folgenden wird 
die Beeinflussung des Messsignal aufgrund des festen Abstandes der Fokuspunkte anhand einer theoretischen Rechnung verdeutlicht. Anschließend zeigt eine experimentelle Untersuchung wie die FLDI-Technik auf Dichteschwankungen außerhalb der Fokusebene reagiert.

\section{Frequenzantwort}

Zunächst sollen die Auswirkungen des finiten Abstandes beider Fokuspunkte betrachtet werden. Als einfacher Ansatz dient die Verwendung einer kosinusförmigen Dichtestörung, die nur in der Fokusebene vorliegt und sich in $x$-Richtung ausbreitet:

$$
\rho(\vec{x}, t)=\cos \left(2 \pi f_{\mathrm{S}} t+k_{\mathrm{S}} x\right) \delta(z)
$$

Hierbei ist $f_{\mathrm{S}}$ die Frequenz der Störung und $k_{\mathrm{S}}$ die entsprechende Wellenzahl, die mittels der Phasengeschwindigkeit $u_{\mathrm{Ph}, \mathrm{S}}=2 \pi f_{\mathrm{S}} / k_{\mathrm{S}}$ ineinander umgerechnet werden können. Anhand von experimentellen Messungen (Weisberger et al. 2019) wird für die Phasengeschwindigkeit von Störungen in Hyperschall-Grenzschichten $u_{\mathrm{Ph}, \mathrm{S}}=0.9 u_{\infty}$ angenommen. Bei $\delta(z)$ handelt es sich um die Delta-Distribution. Zur Vereinfachung wird der Ursprung der Welle auf einen Fokuspunkt gelegt und der zweite Fokuspunkt befindet sich im Abstand $\Delta x=175 \mu \mathrm{m}$. Für $\Delta \rho_{\mathrm{AB}}(z)$ in Gl. 3.14 lässt sich unter der Annahme infinitesimal schmaler Fokuspunkte schreiben:

$$
\begin{aligned}
\Delta s_{\mathrm{opt}} & =K_{\mathrm{GD}} \int \delta(z)\left[\cos \left(2 \pi f_{\mathrm{S}}\left(t+\Delta x / u_{\mathrm{Ph}, \mathrm{S}}\right)\right)-\cos \left(2 \pi f_{\mathrm{S}} t\right)\right] \mathrm{d} z \\
& =K_{\mathrm{GD}}\left[\cos \left(2 \pi f_{\mathrm{S}}\left(t+\Delta x / u_{\mathrm{Ph}, \mathrm{S}}\right)\right)-\cos \left(2 \pi f_{\mathrm{S}} t\right)\right]
\end{aligned}
$$

Mathematisch führt der Abstand der beiden Fokuspunkte zu einer konstanten Phasenverschiebung zwischen den beiden Kosinusfunktionen. Die Amplitude der Fouriertransformation liefert die messbare Signalamplitude:

$$
\left|\mathcal{F}\left(\Delta s_{\text {opt }}\right)\right|(f)=K_{\mathrm{GD}}\left|\sin \left(\frac{\pi f_{\mathrm{S}} \Delta x}{u_{\mathrm{Ph}, \mathrm{S}}}\right)\right|\left[\delta\left(f_{\mathrm{S}}-f\right)+\delta\left(f_{\mathrm{S}}+f\right)\right] .
$$

Die Kosinusfunktionen werden durch die Fourier-Transformation zu den DeltaDistributionen. Die Sinusfunktion in Gl. 3.18 ist eine Folge der konstanten Phasenverschiebung und verursacht eine Reduzierung der Messamplitude abhängig von der Frequenz der Störung. Abbildung $3.17 \mathrm{a}$ zeigt den Verlauf der Sinusfunktion für beliebige $f_{\mathrm{S}}$. Der Verlauf zeigt, dass nur für bestimmte $f_{\mathrm{S}}$ keine Reduzierung der Messamplitude vorliegt. Die maximale Messamplitude ergibt sich, wenn $\Delta x$ ein ungeradzahliges Vielfaches der halben Wellenlänge der Störung ist und somit die Differenz zwischen Wellenberg und Wellental der Kosinusfunktion gebildet wird. Ist $\Delta x$ ein geradzahliges Vielfaches der Wellenlänge, so verschwindet die Messamplitude. Das Antwortverhalten eines idealen Differentialinterferometers ergibt sich aus der Kleinwinkelnäherung der Sinusfunktion und ist in Abb. 3.17a als gestrichelte Linie dargestellt. Für $f_{\mathrm{S}}<1 \mathrm{MHz}$ stimmen die beiden Kurvenverläufe nahezu überein. Für $f_{\mathrm{S}}>1 \mathrm{MHz}$ verliert die Kleinwinkelnäherung aufgrund des endlichen Abstandes zwischen den beiden Fokuspunkten 
a)
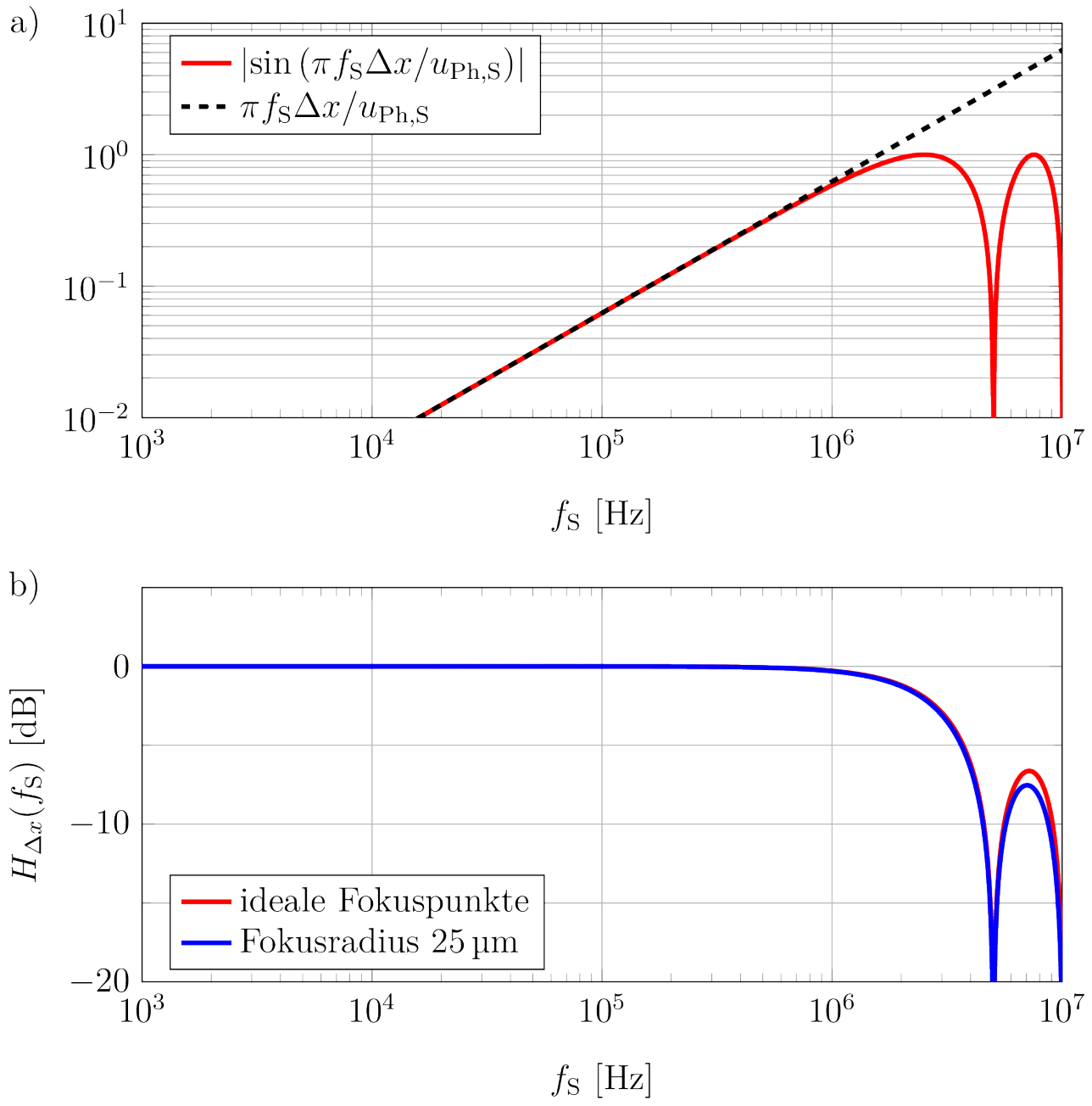

Abbildung 3.17: Antwortverhalten der FLDI-Technik auf eine idealisierte eindimensionale Dichtestörung. Die messbare Signalamplitude ist in a) zusammen mit der Signalamplitude eines idealen Differentialinterferometers dargestellt. Die Systemfunktion aufgrund des endlichen Abstandes der Fokuspunkte ist in b) für einen infinitesimalen und einen endlichen Fokusradius dargestellt.

ihre Gültigkeit und es entsteht eine Reduzierung der Messamplitude gegenüber des idealen Differentialinterferometers. Zur besseren Quantifizierung dieser Abweichung wird die Systemfunktion $H_{\Delta x}$ durch Division der beiden Kurven berechnet und ist in Abb. 3.17b dargestellt. Eine zusätzliche Rechnung für einen endlichen Fokusradius von $25 \mu \mathrm{m}$ mit einer homogenen Intensitätsverteilung zeigt, dass in der Realität die Messamplitude für $f_{\mathrm{S}}>1 \mathrm{MHz}$ zusätzlich reduziert wird. 


\section{Freistrahl Messungen}

Wie die Profilmessungen in Abbildung 3.16 zeigen, ist die Länge des Gebietes, indem die Teilstrahlen der FLDI-Technik getrennt voneinander verlaufen, signifikant größer als die zu untersuchende Grenzschicht. Um zu verstehen, wie die FLDI-Technik auf Dichtestörungen sowohl in der Fokusebene als auch außerhalb reagiert, wurden Messungen mit einem Freistrahl durchgeführt. Zur Erzeugung des Freistrahls wurde eine Düse mit einem Öffnungsdurchmesser von $0.6 \mathrm{~mm}$ verwendet und circa 10 Durchmesser $(6 \mathrm{~mm})$ unterhalb der Fokuspunkte platziert. Beim angelegten Druck von 1.85 bar ist die Austrittsgeschwindigkeit des Freistrahls knapp unterhalb der Schallgeschwindigkeit.

Das Messsignal besteht genau genommen aus einer Faltung zwischen dem sensitiven Volumen der FLDI-Technik und der Größe des Freistrahls. Um das Einflussgebiet des Freistrahl abzuschätzen, wurde die Düse innerhalb der Fokusebene platziert und quer zur optischen Achse verschoben. Für diverse Positionen entlang der $x$-Achse wurden die Schwankungen durch den Freistrahl detektiert. Je stärker die gemessenen Dichteschwankungen zwischen den Teilstrahlen, desto höher ist der Ausschlag des Messsignals und folglich auch der Effektivwert. Innerhalb der Fokusebene ist das sensitive Volumen der FLDI-Technik minimal, sodass zur Vereinfachung angenommen wird, dass die Verteilung der Effektivwerte in Abbildung 3.18a ausschließlich aufgrund der Größe des Freistrahls entsteht. Nach $\pm 2 \mathrm{~mm}$ um den Maximalwert ist der Effektivwert vergleichbar mit dem Grundrauschen der Messtechnik, was einen Eindruck vom Einflussgebiet des Freistrahls vermittelt.

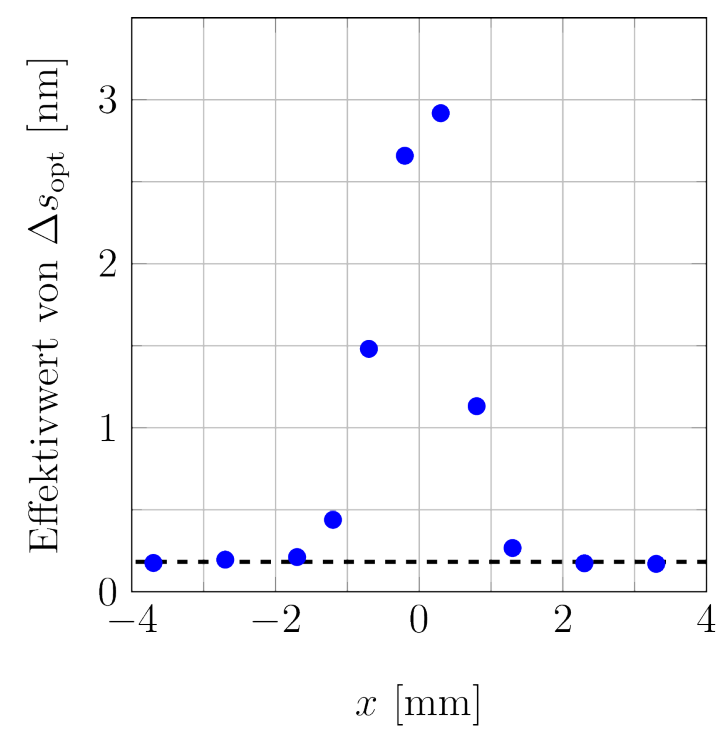

(a) Quer zur optischen Achse innerhalb der Fokusebene

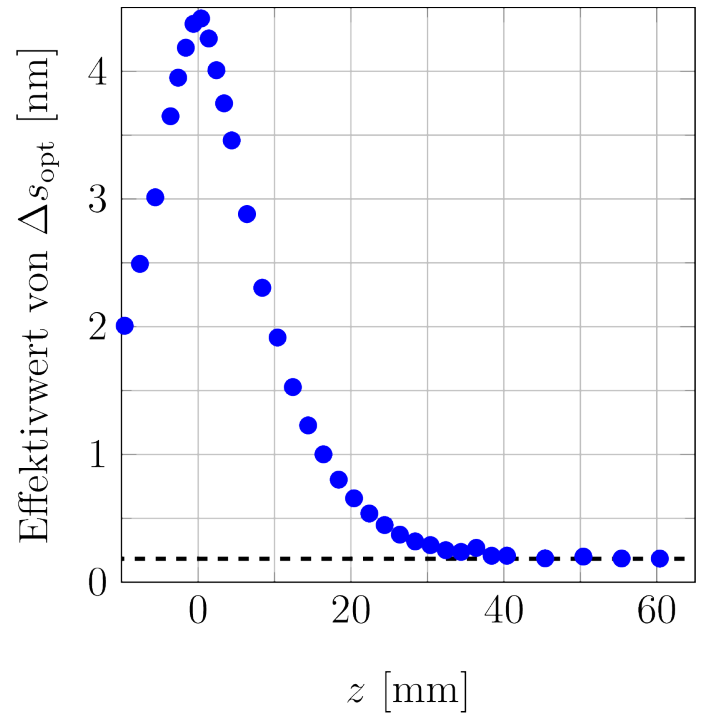

(b) Entlang der optischen Achse

Abbildung 3.18: Effektivwerte der Freistrahl-Messung

Als zweite Messung wurde die Position der Düse entlang der optischen Achse $z$ variiert, um das Antwortverhalten außerhalb der Fokusebene zu untersuchen. Das stärkste 
Messsignal wurde dem Fokuspunkt zugeordnet und auf $z=0 \mathrm{~mm}$ gesetzt. Abbildung 3.19 zeigt das LDS des Freistrahls an diversen Positionen entlang der optischen Achse auf der Empfängerseite des FLDI-Aufbaus. Die LDS zeigen eine eindeutige Tendenz: Je weiter sich der Freistrahl von der Fokusebene entfernt, desto geringer ist die spektrale Leistungsdichte und somit das Antwortverhalten der FLDI-Technik. Außerdem belegt Abbildung 3.19, dass die Dämpfung außerhalb der Fokusebene frequenzabhängig ist. Je höher die Frequenz, desto stärker ist die Dämpfung außerhalb der Fokusebene. Um die Länge des sensitiven Volumens entlang der optischen Achse zu bewerten, wurde erneut der Effektivwert jeder Messposition ermittelt und in Abbildung 3.18b aufgetragen. Nach $12 \mathrm{~mm}$ ist der Effektivwert auf 1/e des Maximalwertes abgefallen und ab $35 \mathrm{~mm}$ sind die Dichtestörungen des Freistrahls nicht mehr vom Grundrauschen zu unterscheiden.



Abbildung 3.19: LDS des Freistrahls an verschiedenen Positionen entlang der optischen Achse auf der Empfängerseite des FLDI-Aufbaus. Die Reduzierung des LDS mit zunehmender Entfernung vom Fokuspunkt belegt die reduzierte Empfindlichkeit der FLDI-Technik außerhalb der Fokusebene

Wie die Analysen zur Frequenzantwort der FLDI-Technik zeigen, beeinflussen die intrinsischen Eigenschaften der FLDI (Abstand der beiden Fokuspunkte und das Strahlprofil entlang der optischen Achse) das messbare Spektrum der Dichtestörungen signifikant. Die vorgestellten Analysen leisten einen wichtigen Beitrag zur Interpretation der Messdaten und ermöglichen die Unterscheidung zwischen strömungsmechanischen Effekten und Artefakten der Messtechnik.

Des Weiteren entstehen bei den Messungen am RWG mechanische Schwingungen, die sich auch auf den optischen Aufbau der FLDI-Technik übertragen und zu einer unerwünschten Phasenverschiebung des Interferometers führen. Testmessungen zeigten, 
dass die mechanischen Schwingungen das Frequenzspektrum bis in den einstelligen Kilohertzbereich verfälschen. Oberhalb dieser Frequenz ist die Amplitude der mechanischen Schwingung kleiner als das Messsignal (vgl. Abb. 4.8). Mit einem Hochpassfilter wurden die Frequenzen unter $500 \mathrm{~Hz}$ herausgefiltert, um den Einfluss der mechanischen Schwingungen in den zeitlichen Darstellungen abzumildern.

\subsection{Messmatrix}

Die vorgestellten Messtechniken ermöglichen die Detektierung unterschiedlicher Strömungsgrößen und liefern zusammen ein umfassendes Gesamtbild der Strömung. Weil nicht alle Größen simultan erfasst werden können, erfordert die sequentiellen Anwendung der Messtechniken eine möglichst exakte Reproduktion der Strömungssituation. Bei der FLDI-Technik ist aufgrund des Schachts die Position der Basisplatte im Windkanal fest vorgegeben. Die Plattenvorderkante befindet sich $2.28 \mathrm{~m}$ stromab vom engsten Querschnitt der Lavaldüse und wird im Folgenden als Pos. A bezeichnet. Nach Ross (1972) beeinflusst die Modellposition innerhalb der Messstrecke die Transitionslage auf dem Modell, weshalb zur Bewahrung der Strömungssituation auch die anderen Messtechniken an der selben Plattenposition angewendet wurden. Eine Übersicht zu den untersuchten Stoßgeneratorpositionen und die jeweils eingesetzten Messtechniken liefert Tabelle A.3.

Aufgrund der optischen Zugänglichkeit am RWG konnten bei Pos. A mittels QIRT nur SGWW im Bereich des Transitionsbeginns vermessen werden. Um auch SGWW bei fortgeschrittener Transition zu untersuchen, wurde die Basisplatte $150 \mathrm{~mm}$ tiefer in die Lavaldüse geschoben (Pos. B). Da sich mit der Plattenposition auch die Transitionslage etwas verschiebt, wurde die ungestörte Strömung erneut aufgenommen. Des Weiteren wurde bei der Plattenposition B der Stoßgenerator um $10 \mathrm{~mm}$ vertikal verschoben ${ }^{1}$, damit der Vorderkantenstoß auch bei stromab gelegenen Stoßauftreffstellen ohne Reflexion unterhalb des Stoßgenerators verläuft (vgl. Kapitel 3.2). Der Umfang der untersuchten Stoßgeneratorpositionen bei Pos. B ist in Tabelle A.4 aufgeführt.

Die Schattenaufnahmen dienen vor allem der Bestimmung der Stoßauftreffstelle. Um eine optimale Sicht auf die einfallende Stoßfront zu erhalten, wurde die Modellposition erneut verändert. Die Schattenaufnahmen der eigentlichen SGWW wurden dabei nicht quantitativ ausgewertet, sodass die Transitionsverschiebung hier unwichtig war. Tabelle A.5 listet die untersuchten Fälle auf.

Insgesamt wurden in dieser Arbeit 153 verschiedene Strömungsbedingungen untersucht und im diesem Zuge 257 Schüsse am RWG ausgewertet.

\footnotetext{
${ }^{1}$ Anhand einer Vergleichsmessung bei identischer Stoßauftreffposition wurde verifiziert, dass die vertikale Verschiebung des Stoßgenerators sich nicht auf die gemessenen Wärmestromdichten auswirkt.
} 


\section{Ergebnisse und Diskussion}

Dieses Kapitel ist in zwei Unterkapitel gegliedert. Das erste Unterkapitel widmet sich der Strömung entlang einer ebenen Platte und fokussiert sich auf die Untersuchung der Transition. Im zweiten Kapitel werden die Auswirkungen der SGWW bei unterschiedlichen Grenzschichtzuständen untersucht. Beide Unterkapitel besitzen die gleiche Struktur. Zunächst werden Schattenaufnahmen verwendet, um die vorliegende Strömungstopologie zu erläutern. Mit Kenntnis der Topologie werden die gemessenen Oberflächenwärmeströme diskutiert. Unter Einbeziehung der zeitaufgelösten Druck- und Dichteschwankungen wird analysiert, welche Störungen innerhalb der Grenzschicht vorliegen. Die gewählte Strukturierung hat den Zweck zunächst die großskaligen Zusammenhänge zu erläutern und schrittweise durch Hinzuziehen weiterer Messdaten ein tiefgreifendes Gesamtbild der Strömungssituation zu liefern.

\subsection{Ungestörte Grenzschicht}

Anhand der Messdaten werden die Auswirkungen der Transition auf die gemessenen Strömungsparamter an der ebenen Platte aufgezeigt und der Einfluss der Einheitsreynoldzahl dargestellt. Die Instabilitätsmessungen ermöglichen einen tieferen Einblick in die physikalischen Vorgänge während der Transition insbesondere bezüglich der zweiten Mack-Mode. Nachdem die Auswirkungen der Transition erläutert und mit den Kenntnissen aus der Literatur verglichen wurden, werden die Messdaten zur Bestimmung des Intermittenzwertes (vgl. Abschnitt 2.1.3) verwendet. Der Intermittenzwert beschreibt den Fortschritt der Transition quantitativ und bildet eine Grundlage für die Analyse der SGWW im zweiten Unterkapitel.

\subsubsection{Schattenaufnahmen}

In Abbildung 4.1 sind gemittelte Schattenaufnahmen von der Umströmung der ebenen Platte für zwei verschiedene $R e_{1}$ dargestellt.

In beiden Schattenaufnahmen sind zwei Hauptmerkmale zu erkennen. Zum einen verläuft oberhalb der Platte ein Verdichtungsstoß. Dieser entsteht an der runden Vorderkante als eine abgelöste Kopfwelle (vgl. Abb. 2.3). Aufgrund der Krümmung des Stoßes variiert sowohl dessen Intensität als auch die Entropiezunahme über den Stoß. Im Bereich der Plattenvorderkante finden die größten Änderungen des Stoßwinkels statt, sodass im wandnahen Bereich eine Entropieschichtung in Form eines veränderten Gesamtdruckprofils vorliegt (Hirschel 2005). Im Sichtbereich der Schattenaufnahme ist die Intensität 


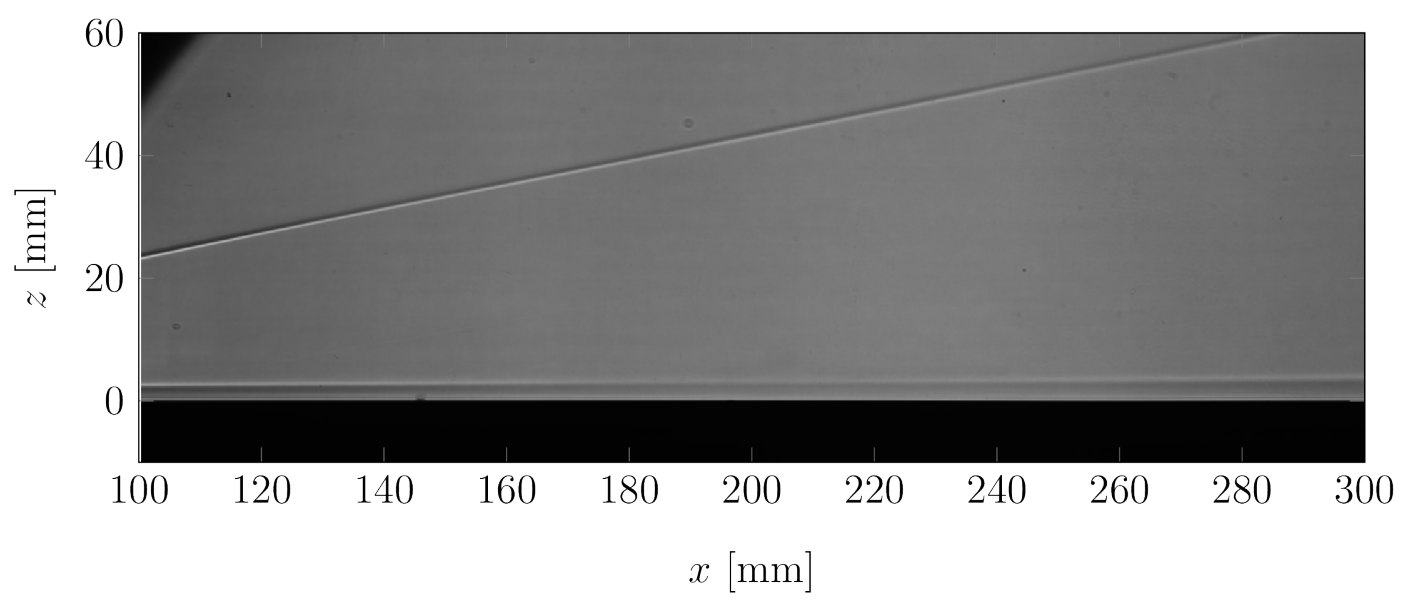

(a) $R e_{1}=8 \cdot 10^{6} \mathrm{~m}^{-1}$

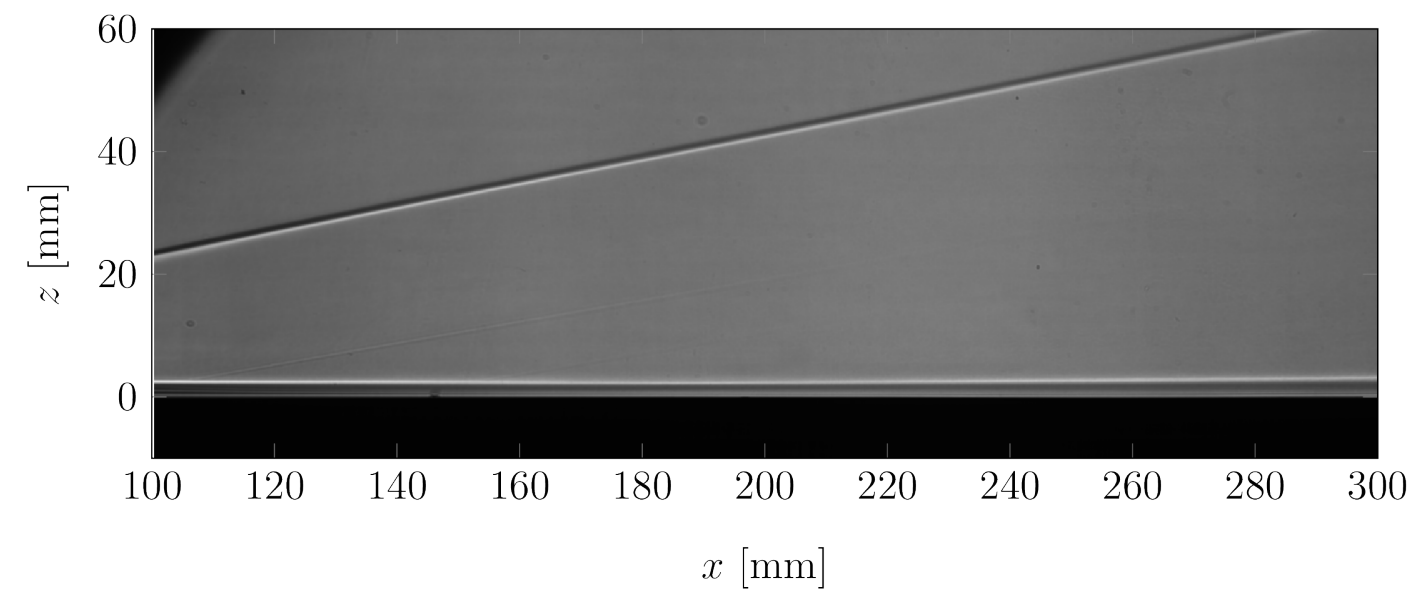

(b) $R e_{1}=24 \cdot 10^{6} \mathrm{~m}^{-1}$

Abbildung 4.1: Über jeweils 200 Einzelaufnahmen gemittelte Schattenaufnahmen von der Umströmung der ebenen Platte für zwei $R e_{1}$

dieser Kopfwelle schwach, da durch den Stoß nur eine Strömungsumlenkung aufgrund des Verdrängungswirkung der Grenzschicht erfolgen muss.

Neben dem Vorderkantenstoß ist in den Schattenaufnahmen auch die Grenzschicht zu erkennen. Nach der idealen Gasgleichung ist bei konstantem Druck das Dichteverhältnis antiproportional zum Temperaturverhältnis. Die Ähnlichkeitslösungen (Abb. 2.1) geben somit eine Vorstellung vom Dichteprofil einer laminaren Grenzschicht. Aufgrund des Dichteprofils entsteht im Schattenbild die sichtbare Intensitätsverteilung der Grenzschicht. Das Anwachsen der Grenzschicht mit der Lauflänge kann in Abb. 4.1a beobachtet werden. Da bei der Schattentechnik das Licht über die gesamte Breite der Messstrecke abgelenkt wird, können auch Randeffekte die gemessene Intensitätsverteilung beeinflussen, weshalb bei der Interpretation der Schattenbilder Vorsicht geboten ist. In Abb. 4.1b zeigt die Intensitätsverteilung für $x<180 \mathrm{~mm}$ keinen Anstieg der Grenzschichtdicke, was 
allerdings unphysikalisch ist. Dieser Fehleindruck könnte durch Sekundärströmungen an den Seitenflächen der ebenen Platte entstehen oder durch Probleme im Strahlengang erzeugt werden, welche allerdings bei Abb. 4.1a keine erkennbaren Auswirkung haben.

In Abb. 4.1b ist aufgrund der größeren $R e_{1}$ der Kontrast erhöht und die Grenzschichtdicke an der selben Position reduziert. Aufgrund des Zusammenspiels beider Effekte ist ein weiterer Verdichtungsstoß erkennbar, der bei $x=108 \mathrm{~mm}$ oberhalb des Grenzschichtrandes sichtbar wird. Der Verdichtungsstoß ist sehr schwach und entsteht vermutlich am Übergang zwischen der Basisplatte und dem Einsatz $(x=96 \mathrm{~mm})$. Obwohl der Einsatz bündig montiert wird, können kleine Spalte nicht komplett ausgeschlossen werden. Da bei $R e_{1}=24 \cdot 10^{6} \mathrm{~m}^{-1}$ eine höhere Dichte vorliegt, können im Schattenbild auch solch schwache Verdichtungsstöße detektiert werden.

\subsubsection{Verteilung der Wärmestromdichte}

Eine typische Oberflächenverteilung der Stanton-Zahl auf der ebenen Platte ist in Abbildung 4.2 dargestellt.

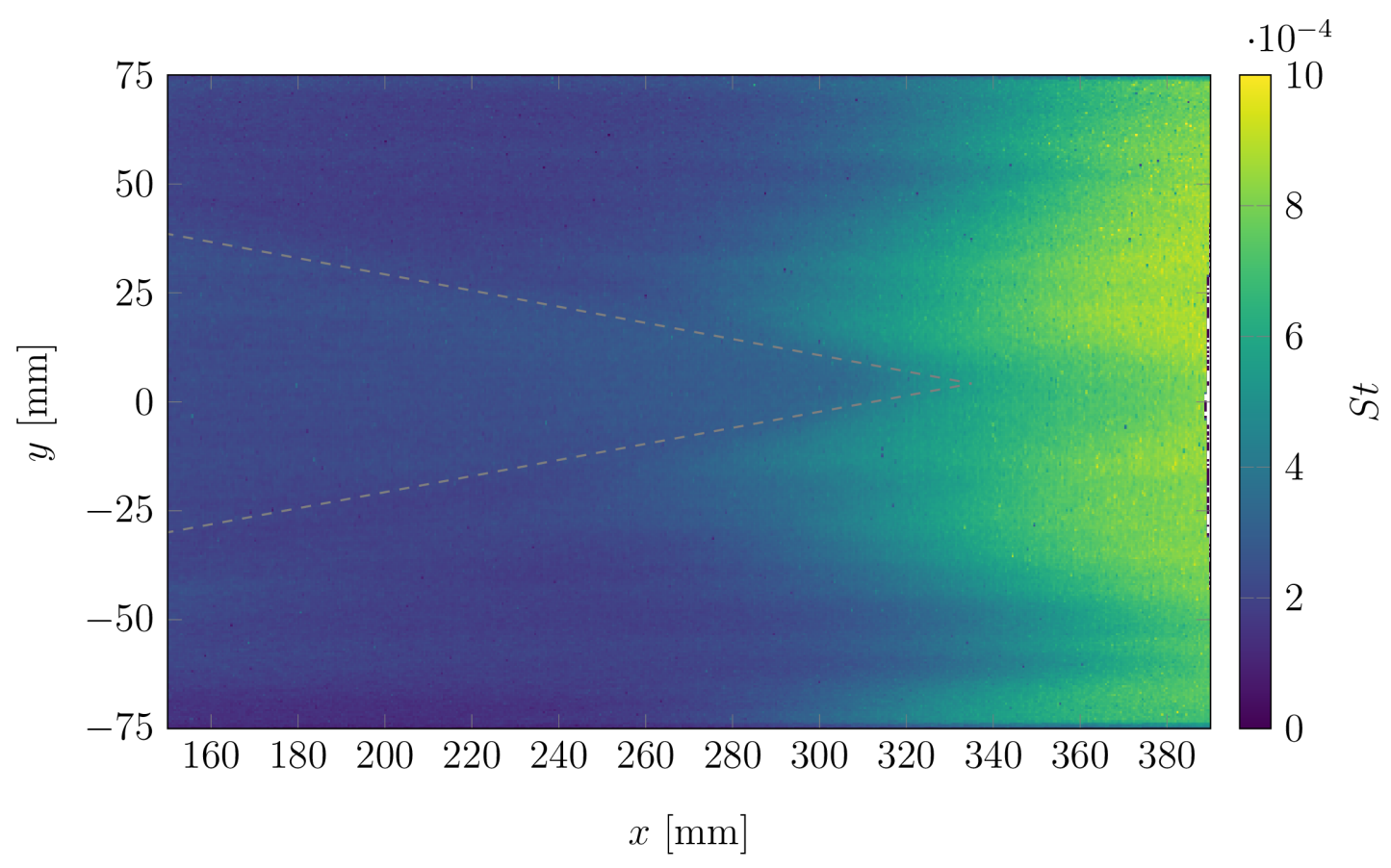

Abbildung 4.2: Oberflächenverteilung der Stantonzahl. Das Einsetzen der Transition ist anhand der steigenden Wärmestromdichte erkennbar. Zusätzlich ist eine keilförmige Struktur zwischen $x \approx 150 \mathrm{~mm}-325 \mathrm{~mm}$ erkennbar. Die dargestellte Verteilung gehört zu Plattenposition B und $R e_{1}=24 \cdot 10^{6} \mathrm{~m}^{-1}$.

Anhand der steigenden Wärmestromdichte ist das Transitionsgebiet zu erkennen. Obwohl eine zweidimensionale Modellgeometrie vermessen wurde, kann eine spannweitige Variation der Stantonzahlverteilung wahrgenommen werden. In der Nähe der Längsachse ist eine keilförmige Struktur erkennbar, die durch grau gestrichelte Linien in Abbildung 
4.2 hervorgehoben wird. Eine Verschiebung der Basisplatte im Windkanal zeigte, dass die Lage des Keils im Bezugssystem der Lavaldüse konstant bleibt. Vermutlich handelt es sich bei dieser Störung um den Fußabdruck eines schwachen Kegelstoßes, der durch fertigungsbedingte Konturabweichungen an einem Verbindungsflansch der Lavaldüse entsteht und in Richtung Düsenachse zusammenläuft. Ein solcher Stoß würde zur Anregung von Störungen innerhalb der Grenzschicht (vgl. Abschnitt 2.1.3) beitragen und somit zur Entstehung einer dreidimensionalen Transitionslage führen.

Auch Willems \& Gülhan (2013) untersuchten die Transitionlage auf einer ebenen Platte anhand der räumlichen Verteilung der Stanton-Zahl. Ihre Experimente zeigten eine vergleichbare spannweitige Abhängigkeit der Transitionslage. Die Autoren sehen die Ursache in ungleichmäßigen Anströmbedingung, die vom radialem Abstand zum Zentrum der rotationssymmetrischen Lavaldüse abhängen (Willems et al. 2015).

Die Stantonzahlverteilung besitzt in erster Näherung eine Achsensymmetrie entlang $y=0 \mathrm{~mm}$ und wird für die weitere Auswertung in spannweitiger Richtung über den Bereich $|y| \leq 25 \mathrm{~mm}$ gemittelt. Die so bestimmte 1D St-Verteilung entlang der ebenen Platte zeigt die natürliche Transition und ist für unterschiedliche $R e_{1}$ in Abbildung 4.3 gegenüber $R e_{x}$ dargestellt. Hinzugefügt wurden die theoretischen Verläufe für die laminare und vollturbulente Grenzschicht, die in Abschnitt 2.1.1 und 2.1.2 hergeleitet wurden. Für die theoretische Abschätzung des vollturbulenten St-Verlaufs beginnt die turbulente Grenzschicht an der Vorderkante der ebenen Platte, weshalb an jeder $R e_{x}$-Position die Grenzschichtdicke etwas überschätzt und konsequenterweise die entdimensionalisierte Wärmestromdichte unterschätzt wird.

Um bei jeder gegebenen $R e_{1}$ eine Wärmestromdichteverteilung entlang des gesamten Plexiglas-Einsatzes zu erhalten, wurden jeweils deren vordere und hintere Hälfte in zwei separaten Windkanalversuchen mittels QIRT vermessen (vgl. Kapitel 3.4). Eine solche Erweiterung des Messgebietes ist ohne Verschiebung der ebenen Platte innerhalb der Lavaldüse möglich, da das Germaniumfenster an zwei diskreten Stellen der Düsenwand montiert werden kann. Trotzdem ergibt sich nur eine unbefriedigende Überlappung der Kurvenverläufe aus zwei getrennten Versuchen. Die statistische Abweichung der St-Verteilung wurde anhand von 5 wiederholten Messungen bei gleichbleibenden Strömungsbedingungen untersucht und besitzt eine Standardabweichung von $2 \%$. Neben der statistischen Streuung wurden im Rahmen der am RWG durchgeführten Parameterstudie auch systematische Abweichungen in den Messdaten festgestellt. In unterschiedlichen Messkampagnen wichen die gemessenen Stanton-Zahlen um bis zu $6 \%$ voneinander ab. Die Auswirkungen dieses systematischen Fehlers sind in Abb. 4.3b im stromab gelegenen Messbereich anhand der jeweils zwei Kurvenverläufe zu sehen. Die Ursache für diese Abweichung konnte bislang nicht geklärt werden. Ohne die Ursache zu kennen, ist eine Wiederholung der Messungen nicht sinnvoll, da aufgrund des Umfangs der Parameterstudie ein erneutes Auftreten dieses systematischen Fehlers nicht ausgeschlossen werden kann. Für die aktuelle Arbeit bedeutet der systematische Fehler eine größere Streuung zwischen den einzelnen Kurven, aber die zugrundeliegenden Trends sind trotzdem erkennbar.

Ein weiterer systematischer Fehler entsteht aufgrund der bereits diskutierten Imperfektion der Anströmung im Windkanal und zeigt sich in Abbildung 4.3b durch eine Erhö- 
hung der Stanton-Zahl innerhalb der laminaren Grenzschicht. Als Konsequenz weichen die laminaren St-Werte vom theoretischen Verlauf ab. Abbildung 4.3a zeigt den Verlauf der Stanton-Zahl für verschiedene $R e_{1}$ bei Plattenposition A. An dieser Plattenposition befindet sich die keilförmige Struktur stromauf des Messbereiches. Die St-Verläufe stimmen signifikant besser mit der laminaren Referenzlösung überein. Die Veränderung der Plattenposition im Kanal zeigt nur eine schwache Auswirkungen auf die Lage der Transition.

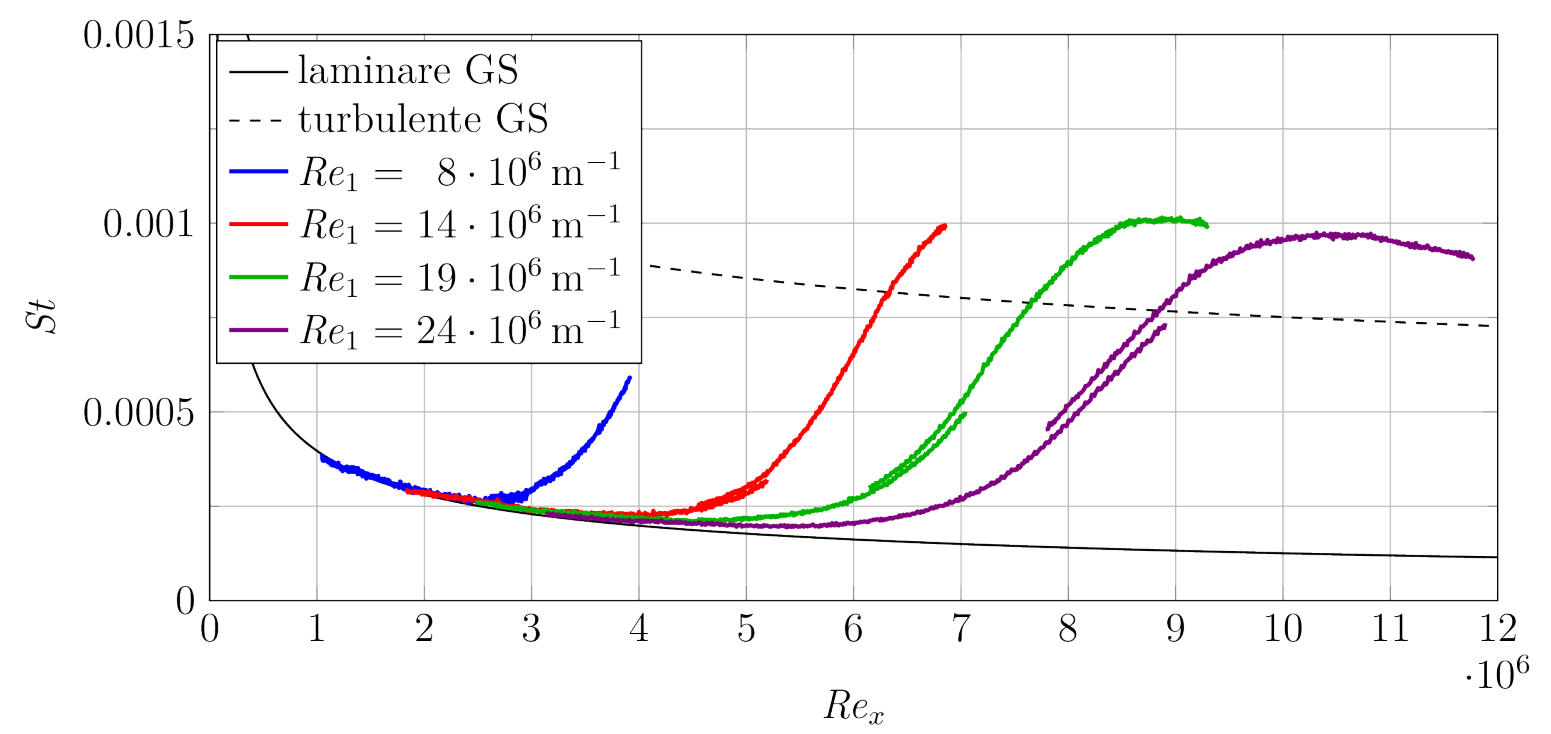

(a) Plattenposition A

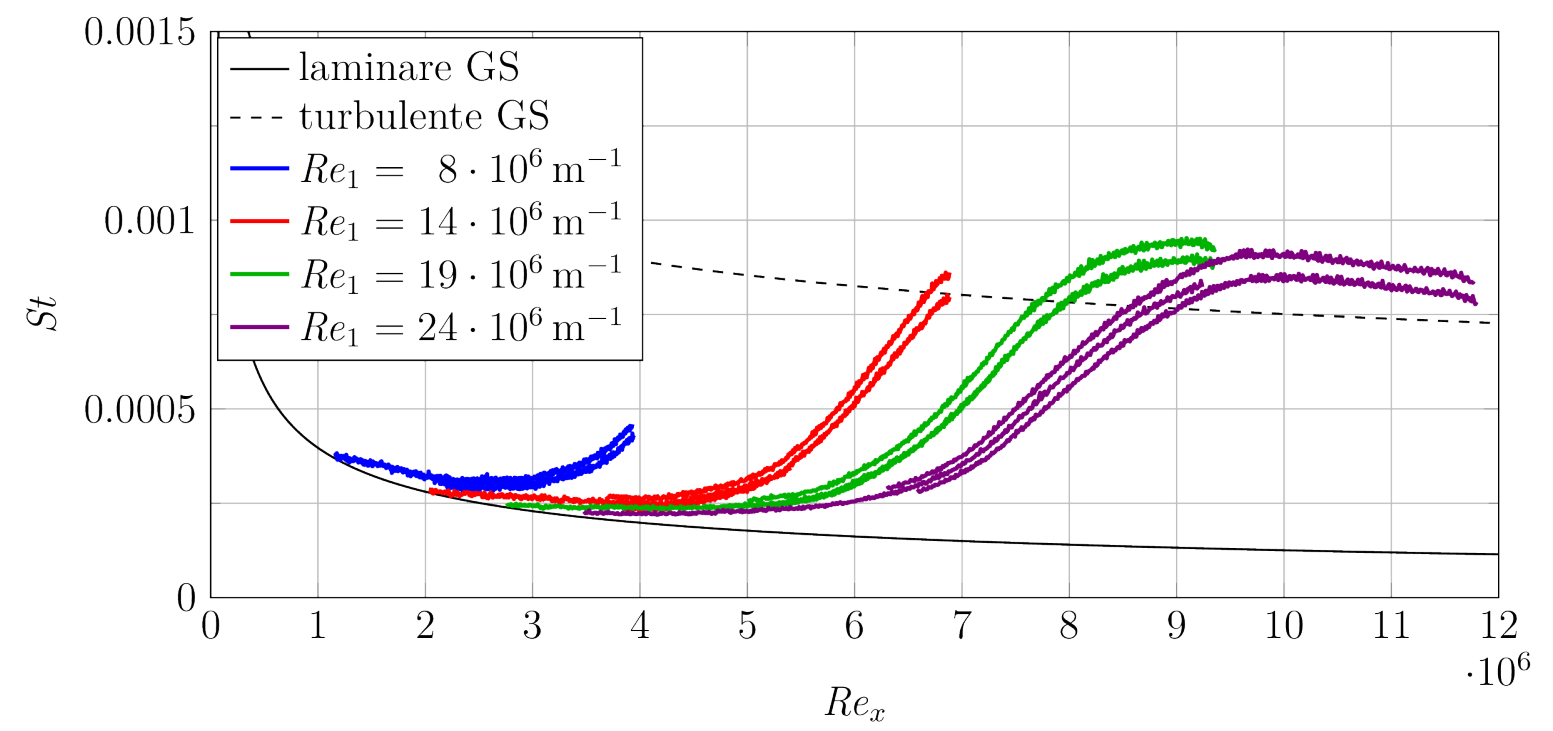

(b) Plattenposition B

Abbildung 4.3: Spannweitig gemittelte Stantonzahlverteilung der natürlichen Transition entlang der ebenen Platte für verschiedene $R e_{1}$ 
Alle experimentellen St-Kurven verlaufen qualitativ ähnlich und beginnen in einer laminaren Grenzschicht. Der signifikante Anstieg der Wärmestromdichte entsteht durch die Transition und führt zu einem lokalem Maximum. Das Maximum wird bei Experimenten oft als Ende der Transition $R e_{\mathrm{Te}}$ interpretiert (Brinich 1956), obgleich an dieser Position noch kein Gleichgewichtszustand innerhalb der Grenzschicht vorliegt (Holden 1977). Stromab des Maximums reduziert sich der Wärmeaustausch innerhalb der Grenzschicht und nähert sich dem Niveau der vollturbulenten Referenzkurve an. Die Abnahme der Wärmestromdichte ist beim Fall $R e_{1}=24 \cdot 10^{6} \mathrm{~m}^{-1}$ sichtbar. Die Kurvenverläufe zeigen den bekannten Effekt (Potter \& Whitfield 1960, Deem \& Murphy 1965, Simeonides 2003), dass mit der Einheitsreynoldszahl auch $R e_{\text {Te }}$ wächst. Nach Pate \& Schueler (1969) hängt $R e_{\mathrm{Te}}$ direkt vom Rauschniveau der Anströmung ab. In Windkanälen entstehen die Schwankungen der Anströmung hauptsächlich durch akustische Wellen, die von der turbulenten Grenzschicht der Windkanalwand ausgesendet werden (Schneider 2008a). Durch eine Änderung von $R e_{1}$, verändert sich auch die Grenzschicht an der Windkanalwand und folglich auch die Fluktuationen in der Anströmung. Der Einfluss von $R e_{1}$ auf die experimentelle Transitionslage verdeutlicht das komplexe Zusammenspiel zwischen äußeren Störungen und der Rezeptivität der Grenzschicht (vgl. Abschnitt 2.1.3). Schülein (2014) zeigte an einer ebenen Platte mit scharfer Vorderkante für die $M a=6$ Bedingungen am RWG, dass die $R e_{1}$-Abhängigkeit der experimentellen Transitionslage mit der Korrelation von Ross (1972) beschrieben werden kann.

\subsubsection{Druckschwankungen}

Wie in Abschnitt 4.1.2 aufgezeigt wurde, ist der Transitionszustand in spannweitiger Richtung nicht einheitlich. Als Konsequenz muss bei der Auswertung der PCB-Sensoren sowohl deren Lage in Hauptströmungsrichtung als auch quer dazu berücksichtigt werden. Abbildung 4.4a verdeutlicht diesen Sachverhalt. Die Abbildung zeigt die St-Verteilung bei $R e_{1}=14 \cdot 10^{6} \mathrm{~m}^{-1}$ an der Plattenposition B. Die Positionen einiger PCB-Sensoren sind als farbige Punkte markiert. Obwohl der rote PCB-Sensor stromab vom orangenen Sensor liegt, ist der Zustand der Transition, erkennbar am Wert der Stanton-Zahl, vergleichbar.

Die spannweitige Variation der Transitionlage erschwert die Analyse und die Zuordnung der erfassten lokalen Druckschwankungen. Die Auswirkungen der Transition auf die Druckschwankungen sind in Abbildung 4.4b zu sehen, wo das Messsignal der Sensoren aus Abb. 4.4 a über die Zeit dargestellt ist. Für die Auftragung wurden die Druckschwankungen skaliert und auf die jeweilige $x$-Koordinate des Sensors verschoben, um die Zuordnung zwischen Messsignal und dem Transitionszustand an der Sensorposition visuell zu unterstützen. Die Zeitsignale bestehen aus Bereichen mit hoher und niedriger Schwankungsamplitude. Kleine Schwankungen liegen in einer laminaren Grenzschicht vor. Strömt ein Turbulenzfleck über den Sensor, so werden deutlich stärkere Schwankungen gemessen. Der hellblaue PCB-Sensor befindet sich im Bereich des Transitionsbeginns. Entsprechend sind hier nur vereinzelte Turbulenzflecken zu erkennen. Die Häufigkeit und Dauer der turbulenten Phasen (Durchgang von Turbulenzflecken) nimmt mit fortschreitender Transition zu. Bei den organgenen und roten Sensoren entspricht das 



Abbildung 4.4: Visualisierung der Transitionslage bei $R e_{1}=14 \cdot 10^{6} \mathrm{~m}^{-1}$ anhand der St-Verteilung und der PCB-Sensorposition (a). Die skalierten Zeitsignale der dazugehörigen PCB-Sensoren zeigen die Turbulenzflecken innerhalb der Grenzschicht bei unterschiedlichen Transitionszuständen (b). Die Auswirkungen der Transition auf das Frequenzspektrum der Druckschwankungen zeigen die LDS (c) 
jeweilige Zeitsignal bereits zu einem Großteil einer turbulenten Strömung.

Die Leistungsdichtespektren der gezeigten Druckschwankungen sind in Abbildung 4.4. dargestellt. Stromauf des Transitionsbeginns (dunkelblauer PCB-Sensor) nimmt das LDS mit der Frequenz ab, da im Messignal keine hochfrequenten Schwankungen durch zweite Mack-Moden oder sogar Turbulenzflecken existieren. Im Bereich des Transitionsbeginns (hellblauer PCB-Sensor) ist bei $f \approx 120 \mathrm{kHz}$ ein Maximum im Leistungsdichtespektrum zu erkennen, der auf die zweiten Mack-Moden zurückgeführt wird. Eine Diskussion bezüglich der Frequenz der zweiten Mack-Moden erfolgt in Kapitel 4.1.4 zusammen mit den Ergebnissen der FLDI.

Mit zusätzlicher Lauflänge häufen sich die Instabilitäten. Gleichzeitig wächst die Amplitude der zweiten Mack-Moden, wodurch über nicht-lineare Effekte zusätzliche Schwingungen und Strukturen angeregt werden. Schließlich brechen diese Strukturen zusammen und Turbulenzflecken entstehen (vgl. Kapitel 2.1.3). Da das LDS aus einer statistischen Mittlung der einzelnen Ereignisse berechnet wird, führt der Transitionsprozess zunächst zu einer Verbreiterung des Maximums, wie es zwischen dem hellblauen und hellgrünen Sensor beobachtet werden kann. Die Verbreiterung nimmt mit der Lauflänge zu, bis letztlich ein breitbandiges LDS (oranger und roter Sensor) vorliegt.

Ein Beispiel für das LDS stromab des Transitionsendes ist in Abbildung 4.5 dargestellt. Hierbei fällt auf, dass das turbulente Spektrum für $f \leq 150 \mathrm{kHz}$ nahezu konstant ist, darüber abfällt und für $f \geq 600 \mathrm{kHz}$ nicht mehr vom Messrauschen zu unterscheiden ist. Der Abfall im LDS wird auch durch eine frequenzabhängige Dämpfung des Messsignals aufgrund der Abmessungen des Drucksensors hervorgerufen. Corcos (1963) berechnete die Frequenzantwort von Drucksensoren mit endlicher Sensorfläche in turbulenten Grenzschichten. Die Ergebnisse für runde Sensoren sind tabellarisch in Abhängigkeit der entdimensionalisierten Frequenz $2 \pi f r / u_{\mathrm{Ph}}$ veröffentlicht. Die Wahl für den Radius $r$ der Messfläche ist bei den eingesetzten PCB-Sensoren nicht eindeutig. Die Messoberfläche des Piezoelementes ist zwar $0.762 \times 0.762 \mathrm{~mm}^{2}$ (Schneider 2019), allerdings befindet sich das Piezoelement unterhalb einer Epoxidschicht, wodurch sich die effektive Messfläche vergrößert. Beresh et al. (2011) wählten für die Aufbereitung ihrer PCB132A31 Messdaten den Wert $r=0.7136 \mathrm{~mm}$, welcher aus den Herstellerabgaben zur effektiven Messoberfläche abgeleitet wurde. Unter Verwendung von $r=0.7136 \mathrm{~mm}$ und $u_{\mathrm{Ph}}=0.9 u_{\infty}$, kann die Frequenzantwort der PCB-Sensoren mit den experimentellen LDS verglichen werden. Für einen direkten Vergleich wurde die theoretische Frequenzantwort nach Corcos (1963) mit $3 \cdot 10^{-8}$ multipliziert und zusammen mit dem experimentellen LDS einer turbulenten Grenzschicht in Abbildung 4.5 dargestellt. Die Frequenzantwort zeigt, dass eine $-3 \mathrm{~dB}$ Dämpfung im LDS bei $f=185 \mathrm{kHz}$ vorliegt und die Sensorfläche der PCB-Sensoren damit zu groß ist, um die Druckschwankungen bei den hohen Frequenzen ungedämpft zu messen. Als Konsequenz kann mit den PCB-Sensoren nur ein Ausschnitt des vollständigen turbulenten Spektrums gemessen werden, dessen Verlauf aufgrund der frequenzabhängigen Dämpfung noch zusätzlich verfälscht ist. Somit erübrigen sich Vergleiche zu theoretischen Vorhersagen bezüglich des LDS bei kompressiblen turbulenten Grenzschichten.

Die transitionsbedingten Veränderungen der Druckschwankungen können auch durch eine Variation von $R e_{1}$ untersucht werden. Abbildung 4.6 zeigt das Messsignal sowie das 




Abbildung 4.5: LDS der Druckschwankungen in einer turbulenten Grenzschicht $(x=$ $473.3 \mathrm{~mm}$ bei $\left.R e_{1}=24 \cdot 10^{6} \mathrm{~m}^{-1}\right)$. Dazu ist die Frequenzantwort der PCB-Sensoren innerhalb einer turbulenten Grenzschicht basierend auf den Rechnungen von Corcos (1963) dargestellt.

Leistungsdichtespektrum vom hellblauen Sensor $(x=346.67 \mathrm{~mm})$ aus Abbildung 4.4 bei verschiedenen $R e_{1}$. Da es sich bei allen $R e_{1}$ um den selben Sensor handelt, ist der Einfluss der mechanischen Vibration (vgl. Abschnitt 3.5.1), das Grundrauschen und die Sensitivität konstant, wodurch die Strömungseffekte etwas einfacher zu analysieren sind. Bei einem Anstieg der Einheitsreynoldszahl verschiebt sich die Transitionslage stromauf, wodurch an einer festen Messposition der Transitionsfortschritt zunimmt. Diese $R e_{1}$-Abhängigkeit belegen sowohl die zeitlichen Messsignale anhand der Häufigkeit an Turbulenzflecken als auch die LDS durch die Ausbildung eines breitbandigen Frequenzspektrums im Zuge der Transition.

Bei den LDS handelt es sich um zeitlich gemittelte Frequenzspektren. Für die Analyse der Transition ist allerdings auch interessant, wie sich das Frequenzspektrum über die Zeit verändert. Daher wurde für einen Messabschnitt (200 ms $\leq t \leq 210 \mathrm{~ms}$ ) aus Abb. 4.6a eine Wavelet-Transformation durchgeführt und in Abb. 4.7 dargestellt. Das Durchqueren von einzelnen Instabilitäten bzw. Turbulenzflecken oberhalb der Sensorfläche äußert sich im Messsignal als diskrete bzw. breitbandige Wellenpakete. Mit der Wavelet-Transformation kann sowohl die Häufigkeit der Wellenpakete als auch der Zeitpunkt ihres Passierens sowie deren jeweilige Bandbreite analysiert werden.

Das Wavelet-Spektrum bei $R e_{1}=8 \cdot 10^{6} \mathrm{~m}^{-1}$ (Abb. $4.7 \mathrm{~b}$ ) ist über die Zeit fast gleichbleibend und zeigt eine Reduzierung der Wavelet-Amplitude mit der steigender Frequenz. Einzelne Events, wie das Passieren von zweiten Mack-Moden, sind nicht zu erkennen, was einer vollständig laminaren Grenzschicht entspricht.

Bei $R e_{1}=14 \cdot 10^{6} \mathrm{~m}^{-1}$ (Abb. 4.7c) sind schmalbandige Wellenpakete in der WaveletTransformation erkennbar (z.B. bei $t=202 \mathrm{~ms}$ oder $t=204.5 \mathrm{~ms}$ ), welche aufgrund der Frequenz (vgl. Kapitel 4.1.4) und geringen Bandbreite als zweite Mack-Moden identifiziert werden können. Deren Amplitude hängt sowohl von der Wachstumsrate inner- 
a)
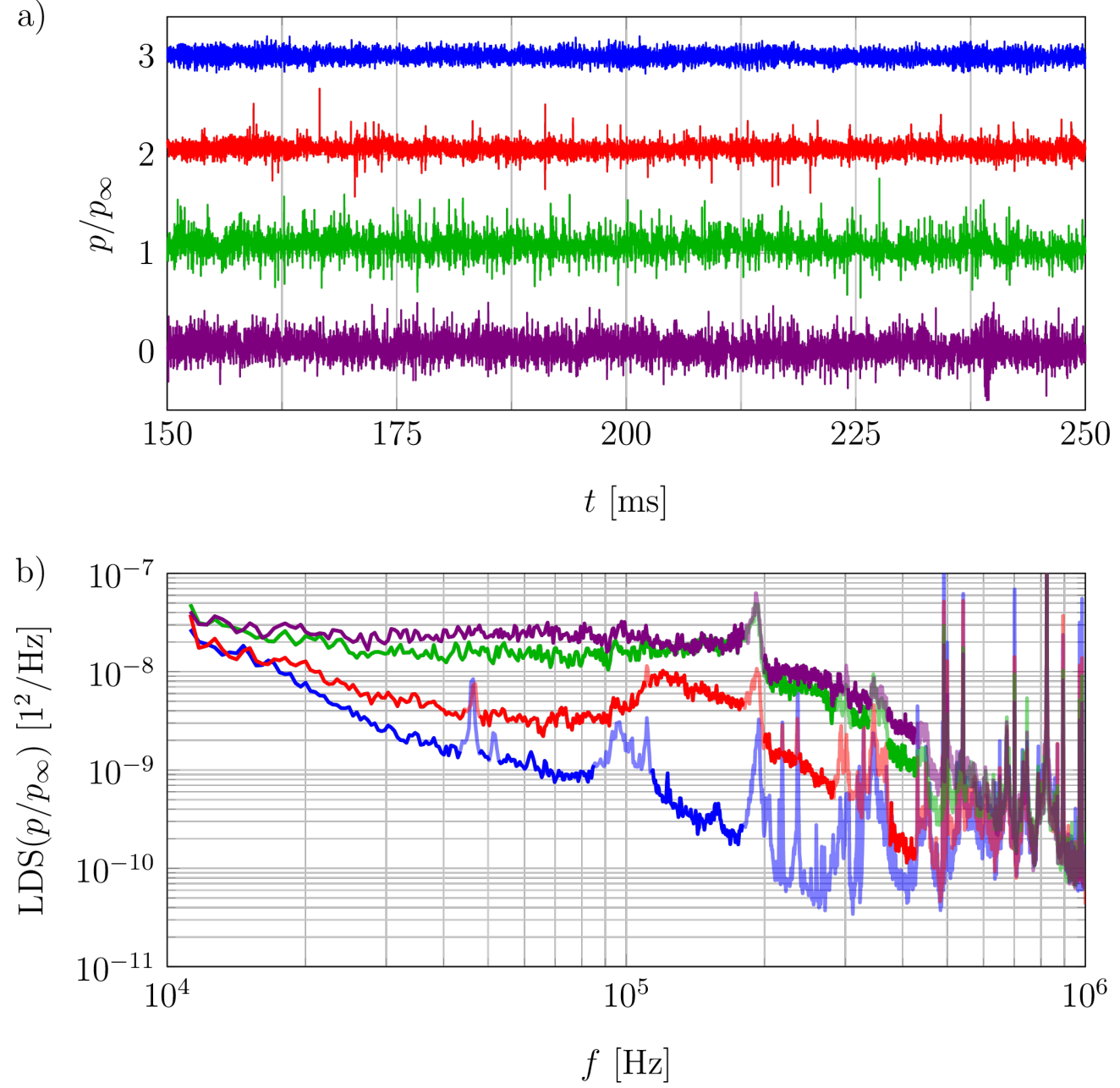

Abbildung 4.6: Zur Übersichtlichkeit wurde der zeitlicher Verlauf der Druckschwankungen bei unterschiedlichen $R e_{1}$ zueinander versetzt (a). Die LDS verdeutlichen wie sich der Transitionszustand an einer festen Messposition aufgrund der $R e_{1}$ verändert (b).

halb der Grenzschicht ab als auch von der Entfernung zwischen dem Entstehungsort der Mode und der Messposition des PCB-Sensors. Als Konsequenz zeigt die WaveletTransformation eine signifikante Streuung zwischen den einzelnen Wavelet-Amplituden der Moden.

Bei $R e_{1}=19 \cdot 10^{6} \mathrm{~m}^{-1}$ (Abb. $\left.4.7 \mathrm{~d}\right)$ sind viele Instabilitäten soweit gewachsen, dass es zum Zusammenbruch der Struktur kam und Turbulenzflecken entstehen. Die Turbulenzflecken sind in der Wavelet-Transformation anhand des breitbandigen Frequenzspektrums erkennbar. Allerdings gibt es noch Zeitbereiche, in denen eine laminare Strömung vorliegt. 
a)

$$
\begin{aligned}
& -R e_{1}=8 \cdot 10^{6} \mathrm{~m}^{-1}-R e_{1}=19 \cdot 10^{6} \mathrm{~m}^{-1} \\
& -R e_{1}=14 \cdot 10^{6} \mathrm{~m}^{-1}-R e_{1}=24 \cdot 10^{6} \mathrm{~m}^{-1}
\end{aligned}
$$


Abbildung 4.7: Zeitlicher Verlauf der Druckschwankungen bei unterschiedlichen $R e_{1}$ (a) und die dazugehörigen Wavelet-Transformationen (b-e). 
Mit einer weiteren Erhöhung der Einheitsreynoldszahl auf $R e_{1}=24 \cdot 10^{6} \mathrm{~m}^{-1}$ (Abb. 4.7 ) verringert sich der prozentuale Anteil der laminaren Strömung. Das Verhältnis zwischen laminarer Strömung und Turbulenzflecken dient als Indikator für den Fortschritt des Transitionsprozesses. Da auch bei $R e_{1}=24 \cdot 10^{6} \mathrm{~m}^{-1}$ noch laminare Bereiche existieren, ist die Transition noch nicht vollständig abgeschlossen. Die Wavelet-Amplitude der Turbulenzflecken ist kleiner als die Wavelet-Amplitude bei der Anregung einzelner Instabilitäten (siehe Abb. 4.7F). Die Abnahme der Störungsamplitude nach dem Zusammenbruch der Instabilität zu einem Turbulenzfleck ist ein bekannter Effekt beim laminar-turbulenten Umschlag (Schneider 2015).

Die Wavelet-Transformation offenbart durch die zeitaufgelöste Untersuchung des Frequenzspektrums, dass es sich bei der Transition um einen stochastischen Prozess handelt der sich aus vielen einzelnen Events zusammensetzt. Die Intensität und das Frequenzspektrum der Events variiert und deren zeitlich gemittelte Verteilung kann anhand der LDS analysiert werden. Dabei setzen sich die Peaks der zweiten Mack-Mode aus wenigen Einzelevents mit vergleichsweise starker Amplitude zusammen, während das Spektrum der turbulenten Strömung aus einer kontinuierlichen Abfolge von breitbandigen Störungen mit schwächerer Intensität besteht.

\subsubsection{Dichteschwankungen}

Die Untersuchung der Dichteschwankungen innerhalb der Grenzschicht ist eine sinnvolle Erweiterung der im vorigen Kapitel diskutierten Druckschwankungen, da die Dichteschwankungen mittels FLDI nichtinvasiv gemessen wurden und die FLDI eine größere Bandbreite besitzt. Allerdings verdeutlichten die Vortests in Kapitel 3.5.2, dass die FLDI-Technik auch auf Dichtestörungen außerhalb der Fokusebene reagiert. Der sensitive Bereich erstreckt sich bis zu $L= \pm 35 \mathrm{~mm}$ entlang der optischen Achse. Da die Grenzschichtdicke im Experiment nur im einstelligen Millimeterbereich liegt, werden dadurch auch die Dichtestörungen in der Außenströmung detektiert. Das Messsignal setzt sich somit aus den Dichteschwankungen beider Bereiche zusammen. Um die Effekte beider Regionen möglichst auseinander halten zu können, wurde mittels FLDI die Anströmung in der leeren Messstrecke (ohne Modell) separat gemessen. Die Fokusebene der FLDI wurde mittig in der $0.5 \mathrm{~m}$ breiten Messstrecke platziert, sodass das Messsignal nicht durch die Grenzschicht an der Windkanalwand beeinflusst wurde. Somit erfolgt eine nichtinvasive Messung der Dichteschwankungen in der Anströmung. Abbildung 4.8a zeigt die gemessenen Leistungsdichtespektren für diverse $R e_{1}$. Die Amplitude der Dichteschwankungen nimmt mit steigender $R e_{1}$ zu und führt zu der gestaffelten Verteilung der einzelnen Spektren. Der Verlauf der Spektren ist qualitativ sehr ähnlich. Im Bereich $f \approx 1 \mathrm{kHz}-3 \mathrm{kHz}$ können die in Abschnitt 3.5 .2 erwähnten Effekte bezüglich der Vibrationen des FLDI Aufbaus beobachten werden. Darüber dominieren die strömungsmechanischen Dichteschwankungen das Messsignal. Bei $f \approx 10 \mathrm{kHz}-20 \mathrm{kHz}$ werden die maximalen Schwankungsamplituden gemessen. Für $f>30 \mathrm{kHz}$ fällt die Signalamplitude stetig bis auf das Messrauschen ab. Für $R e_{1} \geq 10 \cdot 10^{6} \mathrm{~m}^{-1}$ ist bei $f \approx 130 \mathrm{kHz}$ ein Knick im Verlauf der LDS sichtbar. Der Grund für diese Änderung ist nicht bekannt. Da die Amplitude der Störung mit $R e_{1}$ wächst, ist das Messsignal für $R e_{1}=4 \cdot 10^{6} \mathrm{~m}^{-1}$ 
bereits bei $f=500 \mathrm{kHz}$ identisch zum Grundrauschen und für $R e_{1}=24 \cdot 10^{6} \mathrm{~m}^{-1}$ erst bei $f=1 \mathrm{MHz}$.



(a) Mit $R e_{1}$ steigt auch die Amplitude der Dichteschwankungen.

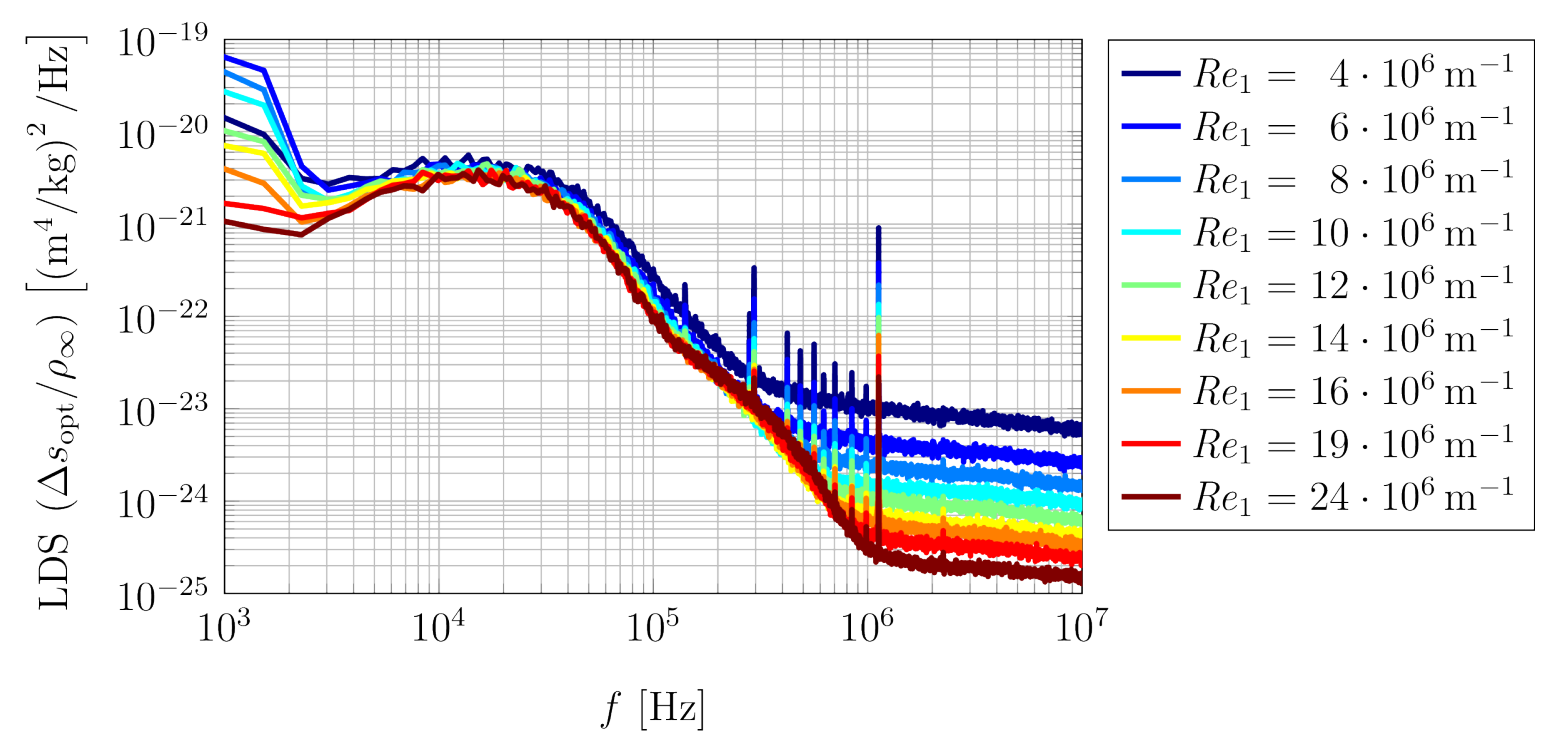

(b) Eine Skalierung mit $\rho_{\infty}$ führt zu einer einheitlichen Verteilung

Abbildung 4.8: Mittels FLDI gemessene Leistungsdichtesprektren der Anströmung für mehrere $R e_{1}$

Eine Skalierung von $\Delta s_{\text {opt }}$ mit der Dichte der Anströmung $\rho_{\infty}$ führt in den Leistungsdichtespektren (Abb. 4.8b) zu einer nahezu einheitlichen Darstellung der Dichteschwankungen in der Anströmung. Lediglich $R e_{1}=4 \cdot 10^{6} \mathrm{~m}^{-1}$ liegt über dem gemeinsamen Verlauf. Pitotdruckmessungen der Anströmung (Schülein \& Wagner 2015) zeigten bereits, dass die prozentualen Schwankungen bei kleinen $R e_{1}$ überproportional groß ausfallen 
und bestätigen die Abweichung für $R e_{1}=4 \cdot 10^{6} \mathrm{~m}^{-1}$.

Es ist mittlerweile gut bekannt, dass die Schwankungen in der Anströmung durch die Entstehung von akustischen Wellen innerhalb der turbulenten Grenzschicht der Lavaldüse und deren Ausbreitung in Richtung Düsenmitte hervorgerufen werden. Ein einfaches Modell für die Erklärung und Beschreibung der akustischen Wellen wurde in der Arbeit von Schopper (1984) behandelt. In dem Modell wird die turbulente Grenzschicht als ein inhomogenes Gebiet betrachtet, in dem kohärente Strukturen existieren, deren Strömungsgeschwindigkeit sich von der lokalen Durchschnittsgeschwindigkeit unterscheidet. Diese Strömungs- bzw. Impulsdifferenz ist der Ursprung für die Entstehung von Druckwellen, die sich im Fluid ausbreiten. Beträgt der Geschwindigkeitsunterschied zwischen der kohärenten Struktur und der Außenströmung mindestens Schallgeschwindigkeit, entstehen schwache Verdichtungsstöße („Shocklets“). Da die kohärente Struktur innerhalb der Grenzschicht mitfließt, ist der Verdichtungsstoß nicht ortsfest, sondern wandert stromab. Durch eine Modellierung des Frequenzspektrums, der Häufigkeit und der Intensität von solchen Verdichtungsstößen, konnten erfolgreich experimentelle LDS der Anströmung nachgebildet werden (Schopper 1984).

Die Messdaten zur Grenzschicht an der ebenen Platte sind in Abbildung 4.9 für zwei $R e_{1}$ an diversen $x$ Positionen dargestellt. Das LDS für $R e_{1}=14 \cdot 10^{6} \mathrm{~m}^{-1}$ zeigt Abb. 4.9a. Für $x \leq 346 \mathrm{~mm}\left(R e_{x} \leq 4.8 \cdot 10^{6}\right)$ überlagern sich die LDS und sind qualitativ identisch zu den Spektren der Anströmung. Anhand der St-Verteilung aus Abb. 4.3a ist bekannt, dass ab $R e_{x}=4 \cdot 10^{6}$ erste transitionsbedingte Abweichungen zur laminare Grenzschicht stattfinden und erst ab $R e_{x} \approx 5 \cdot 10^{6}$ erfolgt eine signifikante Änderung der Stanton-Zahl. Nach Definition der laminaren Grenzschicht (Kapitel 2.1.1) besitzen die Störungen eine vernachlässigbare Amplitude und folglich werden in Abb. 4.9a für $x \leq 346 \mathrm{~mm}$ nur Dichteschwankungen außerhalb der Grenzschicht detektiert. Daher ist der Verlauf der LDS analog zu den Spektren aus Abb. 4.8b. Erst wenn die Amplitude der Störungen innerhalb der Grenzschicht wächst und im Zuge der Transition eine Stärke erreicht, die über dem Messrauschen bzw. dem Messsignal der Außenströmung liegt, ist eine Veränderung der LDS beobachtbar. Für $x>346 \mathrm{~mm}$ ist das Wachstum der Störungen während der Transition sichtbar und führt zu der gestaffelt anwachsenden Frequenzverteilung.

Die vollständige laminar-turbulente Transition konnte mit FLDI für $R e_{1}=$ $24 \cdot 10^{6} \mathrm{~m}^{-1}$ gemessen werden und ist in Abbildung $4.9 \mathrm{~b}$ dargestellt. Aufgrund der höheren $R e_{1}$ beginnt die Transition bei einer kleineren Plattenkoordinate und vollzieht sich über eine kürzere Distanz. Für die stromauf gelegenen Messpositionen ist das LDS durch die Schwankungen in der Außenströmung definiert. Aber bereits bei $x=271 \mathrm{~mm}$ $\left(R e_{x}=6.5 \cdot 10^{6}\right)$ weichen die Leistungsdichtesprektren von diesem Verlauf ab und wachsen bis $x=396 \mathrm{~mm}\left(R e_{x}=9.5 \cdot 10^{6}\right)$ an. Das LDS von $x=421 \mathrm{~mm}$ zeigt keine signifikanten Veränderungen zur direkt stromauf gelegenen Position, was für eine abgeschlossene Transition an der Stelle $x=396 \mathrm{~mm}$ spricht. Sowohl der Transitionsanfang als auch das Transitionsende nach FLDI stimmen mit dem St-Verlauf in Abbildung 4.3a überein.

Das Spektrum der turbulenten Grenzschicht besitzt über einen großen Frequenzbereich eine relevante Signalstärke und fällt erst für $f>2 \mathrm{MHz}$ drastisch ab, bis das 


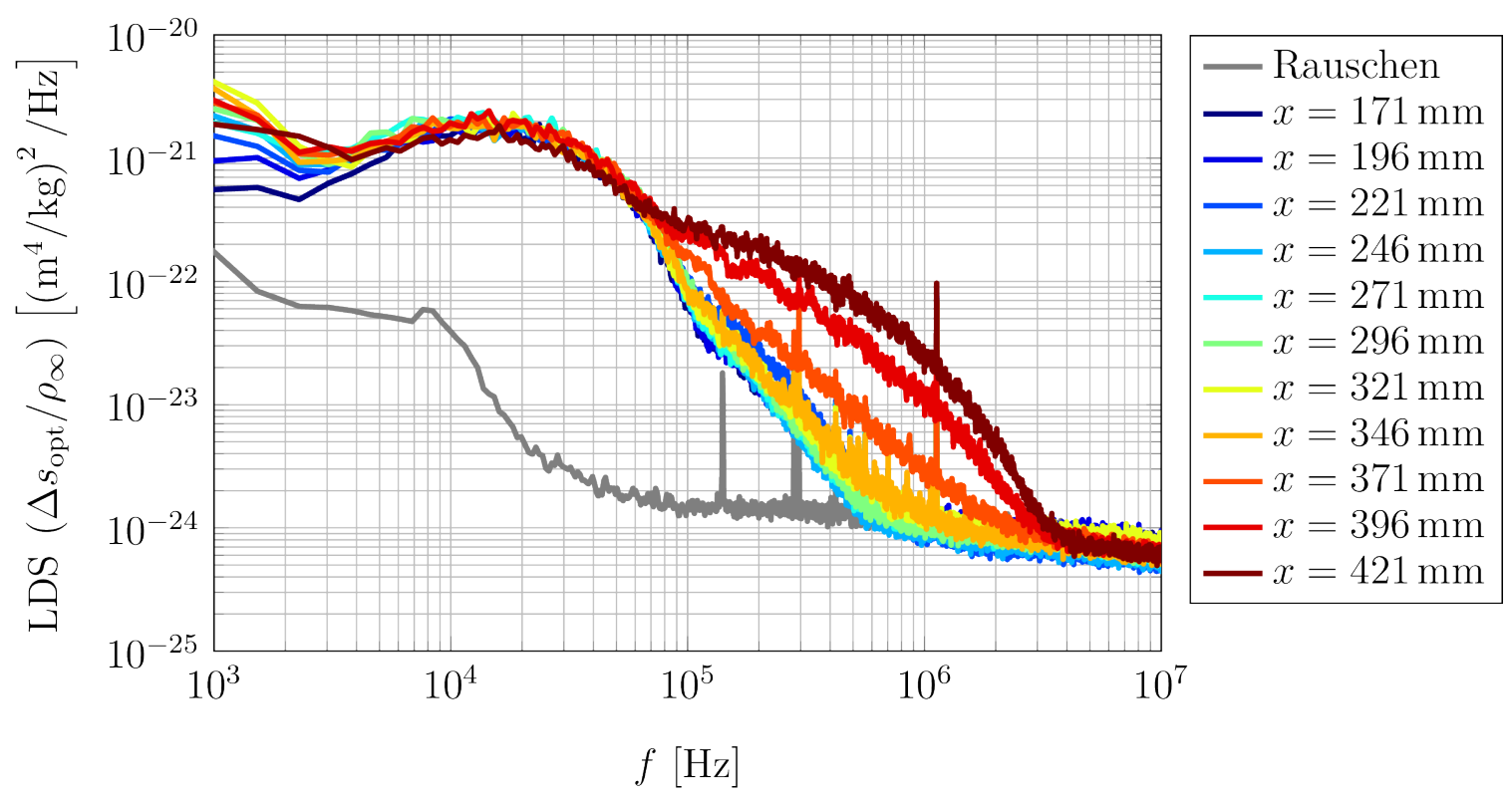

(a) $R e_{1}=14 \cdot 10^{6} \mathrm{~m}^{-1}$

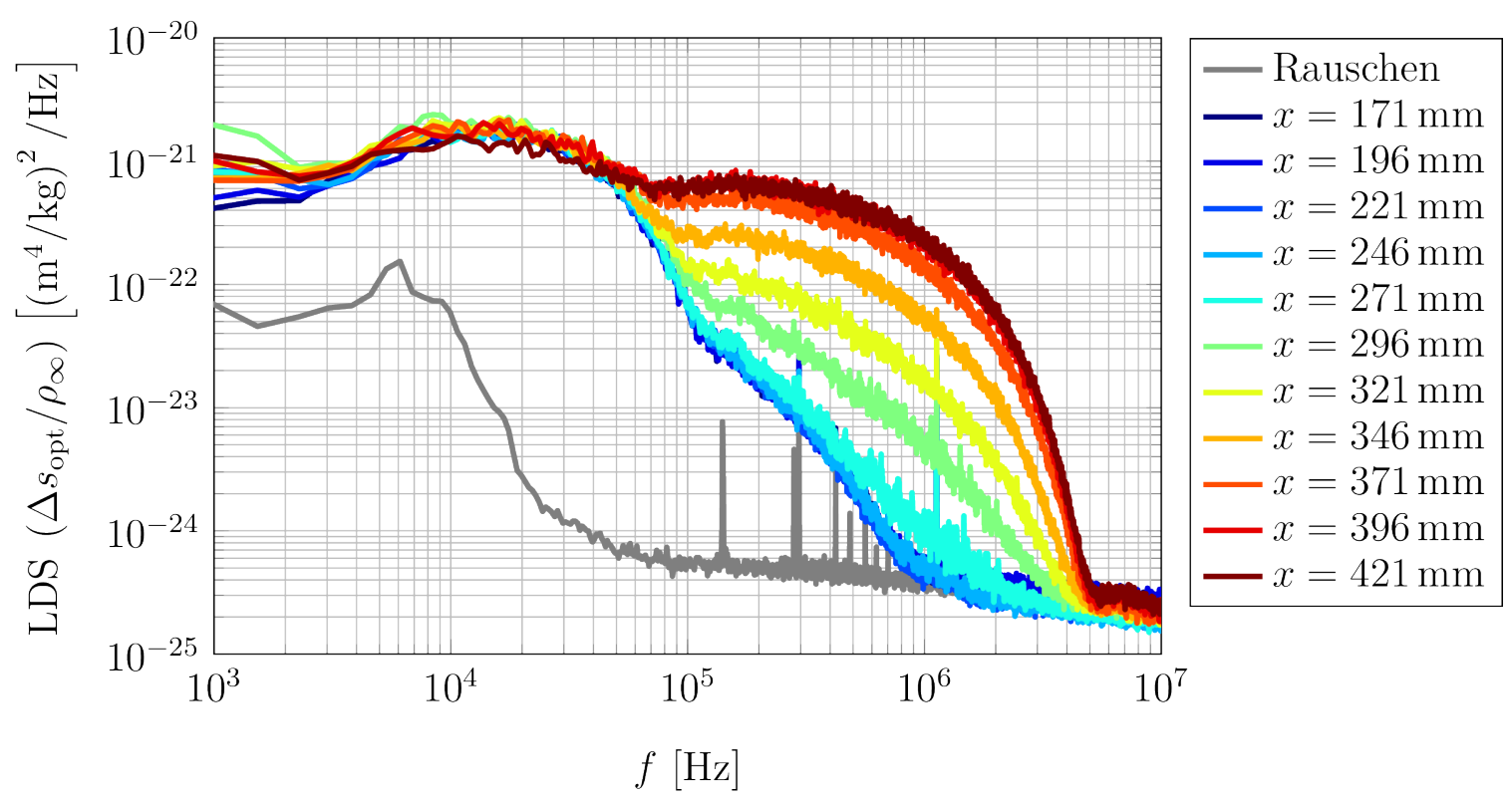

(b) $R e_{1}=24 \cdot 10^{6} \mathrm{~m}^{-1}$

Abbildung 4.9: Leistungsdichtespektren der Dichteschwankungen in der Grenzschicht an verschiedenen $x$-Positionen für zwei unterschiedliche $R e_{1}$. Die Spektren zeigen die Veränderung aufgrund des einsetzenden laminar-turbulenten Umschlags

Niveau des Messrauschens bei $f>5 \mathrm{MHz}$ erreicht wird. Die Frequenz $f=5 \mathrm{MHz}$ stimmt mit der kritischen Frequenz $f_{\text {krit }}$ überein, bei der die FLDI-Technik aufgrund des 
finiten Abstandes der beiden Fokuspunkte nicht auf Störungen reagiert (vgl. Abb. 3.17). Laut Fulghum (2014) und Schmidt \& Shepherd (2015) ist $f_{\text {krit }}$ eine Konsequenz des vereinfachten zweidimensionalen Ansatzes der Störung und sollte in realen Messungen nicht vorliegen. Durch den besonderen Strahlengang ist in den aktuellen Messungen die Annahme einer zweidimensionalen Störung durchaus gerechtfertigt. Die Turbulenzflecken passieren das sensitive Volumen der FLDI direkt in der Fokusebene oder in deren unmittelbarer Nähe. Zusätzlich sind die Fokuspunkte entlang der Strömungsrichtung ausgerichtet, sodass alle theoretischen Annahmen aus Abschnitt 3.5 .2 erfüllt sind. Die Existenz eines unempfindlichen Frequenzbereiches bei der FLDI-Technik kann auch in anderen Arbeiten (Weisberger et al. 2019) anhand eines vergleichbaren Minimums im LDS beobachtet werden, weswegen es sich hierbei wohl nicht um einen spezifischen Effekt des aktuellen Versuchsaufbaus handelt. Allerdings kann die kritische Frequenz bei Weisberger et al. (2019) nicht mit dem aktuellen Ansatz nachgerechnet werden, da deren Strahlengang spannweitig oberhalb der Modelloberfläche verläuft, was die Abschätzung erschwert. Da die Phasengeschwindigkeit der Störung in die Berechnung der kritischen Frequenz eingeht $\left(f_{\text {krit }}=u_{\mathrm{Ph}} / \Delta x\right)$, stellt die experimentelle Vermessung dieser Frequenz vielleicht eine Möglichkeit dar, die Phasengeschwindigkeit von Störungen mittels Standard-FLDI zu bestimmen. Um die Zusammenhänge zwischen der kritischen Frequenz, dem experimentellen FLDI-Aufbau und der Phasengeschwindigkeit $\mathrm{zu}$ verstehen, bedarf es allerdings weiterführende Untersuchungen, die über die Zielsetzung dieser Arbeit hinausgehen.

Da die Grenzschichttransition unter identischen Strömungsbedingungen sowohl mit FLDI als auch mittels PCB-Sensoren untersucht wurde, bietet sich ein direkter Vergleich beider Messtechniken an. Der Vergleich untermauert die beobachteten strömungsmechanischen Effekte und stellt die jeweiligen messtechnischen Eigenschaften heraus. Betrachtet man erneut die LDS in Abb. 4.4c, so sind bei den PCB-Messungen keine Druckschwankungen erkennbar, die mit den Störungen der Außenströmung in Verbindung stehen. Schopper (1984) untersuchte wie akustische Störungen innerhalb der Außenströmung die laminare Grenzschicht an Windkanalmodellen beeinflussen. Dafür wurden die Schwankungen der Außenströmung als eine Abfolge von schwachen Verdichtungsstößen vereinfacht (s. o.) und die Interaktion der Stöße mit der Grenzschicht untersucht. Schopper zeigte für schwache Stöße, dass diese beim Eindringen in die Grenzschicht abgelenkt und oberhalb der Schalllinie fokussiert werden. Diese Fokussierung wird auch als Kaustik bezeichnet und wirkt wie ein Tiefpass-Filter, sodass unterhalb der Kaustik Störungen deutlich gedämpft werden. Da die PCB-Sensoren bündig mit der Oberfläche des Platteneinsatzes abschließen, befinden sich die Sensoren folglich unterhalb der Kaustik und sind zum großen Teil von den Störungen der Außenströmung abgeschirmt.

Da das Messsignal der PCB Sensoren nicht mit Schwankungen aus der Anströmung überlagert ist, besitzen PCB-Sensoren gegenüber FLDI einen deutlichen Vorteil bei der Untersuchung von laminaren Grenzschichten. Auch schwache Störungen in der laminaren Grenzschicht, wie die zweiten Mack-Moden zu Beginn der Transition, werden von den PCB-Sensoren detektiert und sind deutlich im Leistungsdichtespektrum erkennbar (vgl. Abb.4.4c). In den LDS der FLDI-Daten sind keine zweiten Mack-Moden sichtbar, da ver- 
mutlich die Störungen der Außenströmung die schwachen instabilen Moden überdecken. Um diesem Ansatz nachzugehen, wurde das FLDI-Messsignal bei $R e_{1}=14 \cdot 10^{6} \mathrm{~m}^{-1}$ mittels Wavelet-Transformation analysiert (Abb. 4.10).
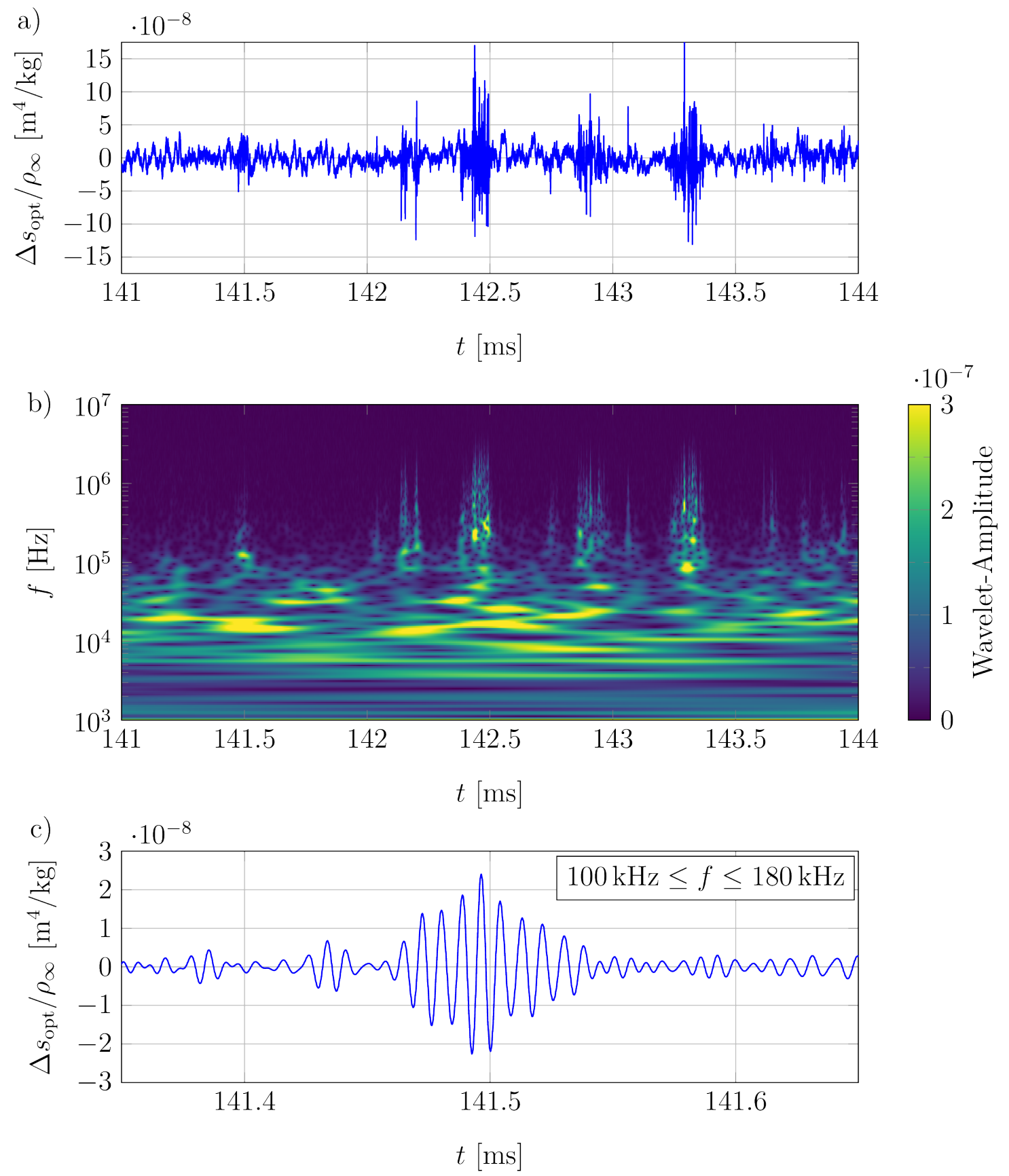

Abbildung 4.10: Zeitlicher Verlauf (a) der FLDI-Messung an der Position $x=393.6 \mathrm{~mm}$ bei $R e_{1}=14 \cdot 10^{6} \mathrm{~m}^{-1}$ und die dazugehörige Wavelet-Transformation (b). Der bandpassgefilterte Ausschnitt (c) von einer zweiten Mack-Mode ähnelt einem Wellenpaket. 
Die Wavelet-Transformation in Abb. 4.10 offenbart, dass bereits viele breitbandige Turbulenzflecken in der Strömung vorliegen, die den gleichmäßigen Frequenzverlauf in Abb. 4.9a hervorrufen. Allerdings ist bei $t=141.5 \mathrm{~ms}$ auch eine schmalbandige Störung erkennbar, deren Frequenz $f=125 \mathrm{kHz}$ beträgt und mutmaßlich eine zweite MackMode repräsentiert. Ein bandpassgefiltertes Messignal dieser Störung ist in Abb. 4.10. dargestellt und zeigt ein für zweite Mack-Moden typisches Wellenpaket (Weisberger et al. 2019). In den FLDI-Messungen von Weisberger et al. (2019) waren die zweiten MackModen bereits im LDS als lokaler Peak erkennbar. Dieser Unterschied zu den Messungen in dieser Arbeit entsteht vermutlich, da bei Weisberger et al. die Grenzschicht einen größeren Anteil des sensitiven Volumens der FLDI-Technik eingenommen hat.

\section{Frequenz der zweiten Mack-Moden}

Nach Demetriades (1977) und Stetson et al. (1989) ist die Wellenlänge und somit auch die Frequenz der zweiten Mack-Mode an die lokale Grenzschichtdicke gekoppelt und ergibt nach der Skalierung $\lambda_{\text {Mack }} / \delta_{99}$ bzw. $u_{\infty} /\left(f_{\text {Mack }} \delta_{99}\right)$ einen konstanten Wert von 2. Um diese Korrelation für die Messungen am RWG zu überprüfen, wurde die WaveletTransformationen genutzt um einzelne zweite Mack-Moden zu identifizieren und deren Frequenz zu bestimmen. Die Identifizierung wurde von Hand durchgeführt, da kein zuverlässiger Algorithmus für die Detektion der zweiten Mack-Moden gefunden werden konnte. Für die Bestimmung der Grenzschichtdicke $\delta_{99}$ wurde eine 2D Simulation von der Umströmung der ebenen Platte durchgeführt. Bei der Simulation wurde der DLRTau-Code (Schwamborn et al. 2006) eingesetzt und eine laminare Grenzschicht sowie eine isotherme Wandtemperatur festgelegt.

Die Auswertung wurde sowohl für die FLDI- als auch für die PCB-Daten durchgeführt. Abhängig vom transitionellen Zustand der Grenzschicht konnte an der jeweiligen Messposition eine unterschiedliche Anzahl an zweiten Mack-Moden für die Auswertung genutzt werden. Abbildung 4.11 zeigt das gemittelte Ergebnis der Skalierung für die einzelnen Messpositionen. Die Fehlerbalken verdeutlichen anhand der $1 \sigma$-Umgebung die Streuung der Messdaten.

Die Abweichung der experimentellen Ergebnisse zum erwarteten Wert 2 liegt innerhalb der Korrelationsgenauigkeit (Demetriades 1977, Stetson et al. 1989), wo die Zahlenwerte für vergleichbare Temperaturverhältnisse $T_{\mathrm{w}} / T_{0}$ zwischen 2 und 2.5 lagen. Wie aus strömungsmechanischer Sicht zu erwarten, liefern die beiden Messtechniken quantitativ sehr ähnliche Ergebnisse. Zwei Messpositionen bei den PCB-Messdaten für $R e_{1}=24 \cdot 10^{6} \mathrm{~m}^{-1}$ widersprechen der Korrelation allerdings deutlich. An diesen Messpositionen liegen die bestimmten Frequenzen deutlich über $180 \mathrm{kHz}$ und befinden sich somit in einem Frequenzbereich, der durch mechanische Vibrationen dominiert ist (vgl. Kapitel 3.5.1). Eine Fehlinterpretiation von mechanischen Schwingung als zweite Mack-Moden oder eine Wechselwirkung zwischen einer zweiten Mack-Mode und einer mechanischen Eigenschwingung kann nicht ausgeschlossen werden, weshalb diese beiden Datenpunkte fragwürdig sind und als offene Symbole gekennzeichnet sind.

Die Verteilung der Datenpunkte in Abb. 4.11a zeigt eine Annäherung der Datenpunkte an $u_{\infty} /\left(f_{\text {Mack }} \delta_{99}\right)=2$ mit zunehmender $R e_{x}$, welche bei Demetriades (1977) und Stetson 


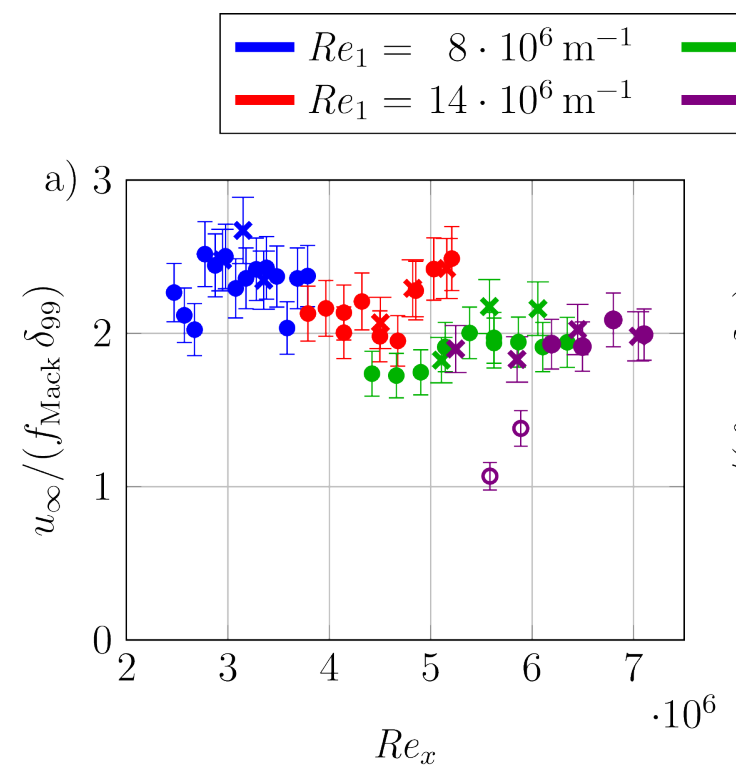

$$
\begin{array}{ll}
R e_{1}=19 \cdot 10^{6} \mathrm{~m}^{-1} & \bullet \mathrm{PCB} \\
R e_{1}=24 \cdot 10^{6} \mathrm{~m}^{-1} & \times \mathrm{FLDI}
\end{array}
$$
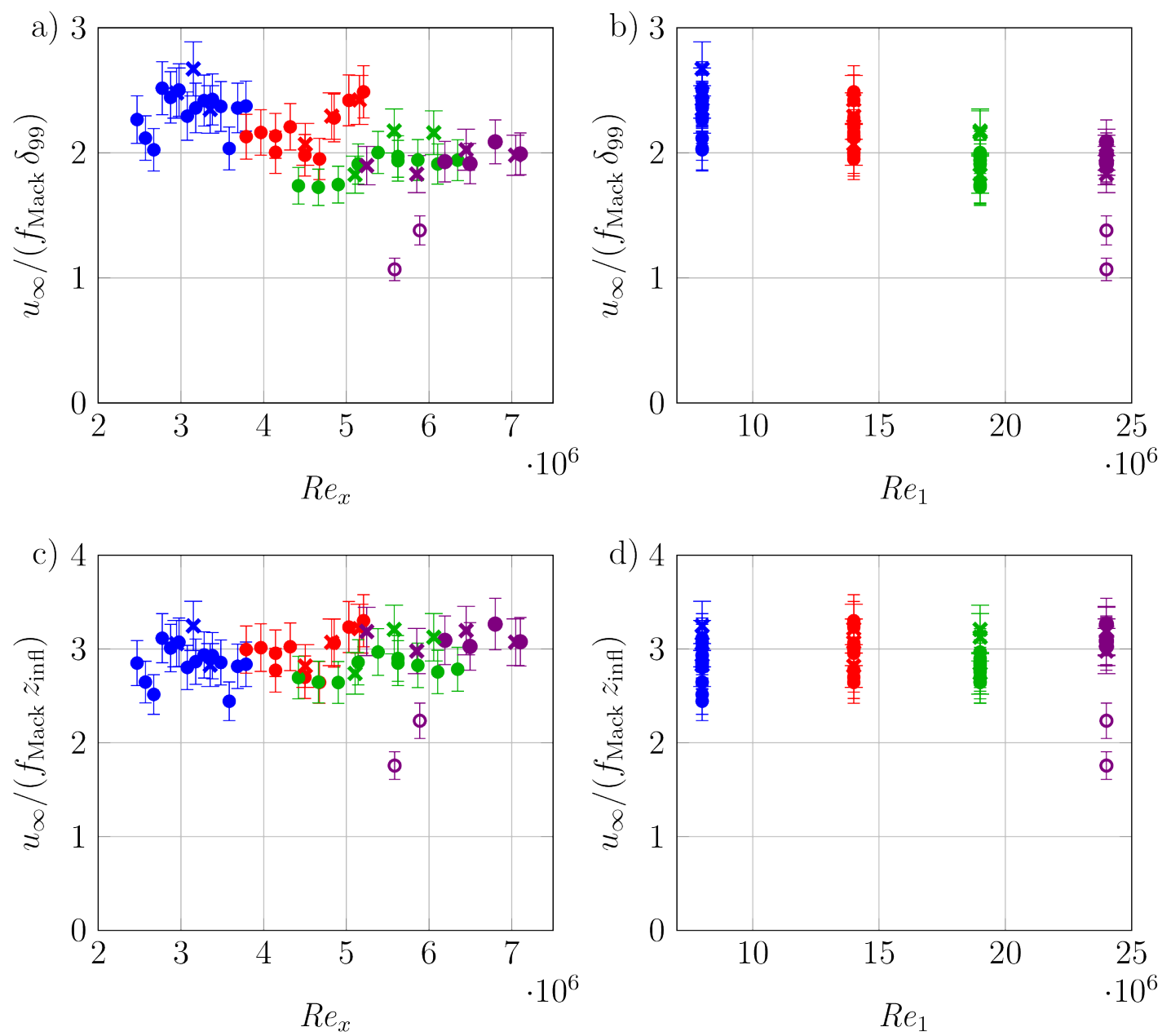

Abbildung 4.11: Bei einem etablierten Skalierungsgesetz (a) für die Frequenz der zweiten Mack-Mode $f_{\text {Mack }}$ verbleibt eine Abhängigkeit bezüglich $R e_{1}(\mathrm{~b})$. Durch die Verwendung einer mechanismusbasierten Längenskala $z_{\text {infl }}$ können die $R e_{1}$-Effekte bei der Skalierung adäquat berücksichtigt werden $(\mathrm{c}, \mathrm{d})$.

et al. (1989) nicht beobachtbar ist. Abb. 4.11b verdeutlicht, dass die Tendenz mit einem $R e_{1}$-Effekt zusammenhängt, der in der Skalierung nicht hinreichend berücksichtigt wird.

Kuehl (2018) analysierte die Entstehung von zweiten Mack-Moden mittels linearer Stabilitätsanalyse und zeigte, dass die berechneten Eigenmoden durch die Ausbildung eines lokalen Impedanz-Minimums zwischen Modellwand und Grenzschichtrand innerhalb der Grenzschicht eingeschlossen sind. Dabei korrelierte die obere Grenze des ImpedanzMinimums mit dem Wendepunkt der akustische Feldimpedanz $Z_{\mathrm{F}}$ der Grundströmung. Die akustische Feldimpedanz beschreibt den Widerstand eines Mediums gegen die Ausbreitung einer akustischen Welle und ist durch $Z_{\mathrm{F}}=\rho c$ gegeben (Meyer \& Neumann 
1974). Die durchgeführten TAU-Simulationen liefern den wandnormalen Abstand $z_{\text {infl }}$ des Wendepunktes der akustischen Feldimpedanz. Wird bei der Skalierung von $f_{\text {Mack }}$ die Grenzschichtdicke $\delta_{99}$ durch das Längenmaß $z_{\text {inf }}$ ersetzt, so belegen Abb. 4.11k und $\mathrm{d}$, dass durch die mechanismusbasierte Skalierung der $R e_{1}$-Effekt besser berücksichtigt wird und die neue Skalierungskonstante keine Abhängigkeit von $R e_{x}$ aufweist.

\subsubsection{Intermittenz}

In der vorangegangenen Diskussion der ungestörten Grenzschicht wurde dargestellt, wie die einsetzende Transition die jeweiligen Strömungsgrößen verändert. Im Umkehrschluss kann diese Veränderung auch dazu verwendet werden, den Transitionszustand zu beschreiben. In der Literatur wird die Transition oft durch den skalaren Intermittenzwert $\gamma$ beschrieben, der an jeder Position den zeitlichen Anteil angibt, in der die Grenzschicht turbulent ist. Der Intermittenzwert spiegelt somit den Fortschritt der laminarturbulenten Transition wieder, wobei ein einzelner Parameter natürlich nicht ausreicht, um den Zustand der Transition adäquat zu charakterisieren. Allerdings stellt $\gamma$ eine sinnvolle und praktikable Möglichkeit dar, den Transitionsfortschritt in Skalierungsgesetzen zu berücksichtigen. Für diesen Verwendungszweck existieren diverse Verfahren, mit denen die Bestimmung des Intermittenzwertes anhand unterschiedlicher Strömungsgrößen möglich ist. Da in der aktuellen Arbeit mehrere Strömungsgrößen zur Transition an der ebenen Platte gemessen wurden, werden im Folgenden etablierte Verfahren zur Intermittenzbestimmung vorgestellt und die Ergebnisse gegeneinander bewertet.

\section{Direkte Intermittenzbestimmung}

Die direkte Methode zur Bestimmung der Intermittenz besteht in der Detektion der turbulenten Flecken in einem Zeitsignal und wird bei den FLDI bzw. PCB Messdaten eingesetzt. Das Vorgehen kann üblicherweise in vier Schritte unterteilt werden (Veerasamy \& Atkin 2020):

1. Zunächst wird das Messsignal aufbereitet, um die Detektion eines Turbulenzfleckens zu erleichtern.

2. Es wird eine Selektionsfunktion $S F(t)$ definiert, anhand dessen die Turbulenzflecken von der laminaren Strömung selektiert werden. Oft wird $S F(t)$ über ein Zeitintervall $T_{\mathrm{M}}$ gemittelt, um einzelne Signalstörungen zu entfernen.

3. Anhand eines Schwellenwertes $S$ wird das Signal von $S F(t)$ einer laminaren bzw. turbulenten Strömung zugeordnet. Wurde eine turbulente Strömung detektiert, wird die Indikatorfunktion $\operatorname{Ind}(t)$ auf 1 gesetzt und sonst gilt $\operatorname{Ind}(t)=0$.

4. Die zeitliche Mittelung von $\operatorname{Ind}(t)$ ergibt den Intermittenzwert $\gamma$.

Obwohl dieses prinzipielle Vorgehen bereits in vielen Arbeiten angewendet wurde, gibt es nach wie vor keine einheitliche Definition zur Berechnung von $S F(t)$. Weitere Unstimmigkeiten liegen in der Wahl des Schwellenwertes und $T_{\mathrm{M}}$. Diese Willkürlichkeit wird in 
mehreren Veröffentlichungen kritisiert und Vorschläge zur Standardisierung des Verfahrens werden unterbreitet (Schneider 1995, Veerasamy \& Atkin 2020). Die unterschiedlichen Ansätze zur Definition von $S F(t)$ gehen letztlich auf das fehlende Verständnis der Transitionmechanismen zurück. Ohne ein tiefes Verständnis, ist es unmöglich eine allgemeingültige Selektionsfunktion zu definieren. Die Intermittenzbestimmung wurde am häufigsten anhand von Zeitsignalen aus Heißfilm- bzw. Hitzdraht-Messungen durchgeführt (Canepa et al. 2002). Die Detektion der turbulenten Spots ist bei diesen Techniken besonders einfach, da sich sowohl die statische Messgröße als auch deren relative Schwankungen innerhalb eines turbulenten Spots erhöhen. Beide Effekte wirken somit multiplikativ, sodass als Selektionsfunktion häufig eine Aufbereitung der Schwankungsamplitude verwendet wird.

Bei den FLDI- und PCB-Messungen in dieser Arbeit erhöht sich innerhalb eines turbulenten Fleckens nur die relative Schwankungsamplitude der Messgröße. Die mittlere statische Messgröße bleibt hingegen unverändert. Als Konsequenz ist eine Selektion aufgrund der Schwankungsamplitude erschwert und wurde anhand von Testauswertungen als unzureichend eingestuft. Um die Detektion der Turbulenzflecken zu verbessern, wurde mittels Wavelet-Transformation eine eigene Selektionsfunktion entwickelt, die neben der erhöhten Schwankungsamplitude innerhalb der Flecken auch das Frequenzspektrum der Störungen berücksichtigt. Obwohl hierdurch eine weitere Variante von $S F(t)$ eingeführt wird, werden diesmal zwei charakteristische Eigenschaften von Turbulenzflecken verwendet, was deren Detektion erleichtert und zuverlässiger macht. Zudem wurden bei der Ausarbeitung des Verfahrens bestehende Konzepte zur Standardisierung aufgegriffen und berücksichtigt.

Im Folgenden wird die Methode anhand eines Beispiels erläutert. Abbildungen 4.12 a,b zeigen einen Ausschnitt des FLDI-Messsignals und die dazugehörige WaveletTransformation.

In beiden Darstellungen sind Turbulenzflecken erkennbar, allerdings erschweren Störsignale, wie die Schwankungen in der Außenströmung, eine computergestützte Detektion der Turbulenzflecken. Für die Intermittenzbestimmung empfiehlt Schneider (1995) eine Skalierung der Messdaten mit dem Messsignal aus der laminaren Grenzschicht, wodurch auch intrinsische Störungen der Messtechnik wie elektronisches Rauschen oder Vibrationen berücksichtigt und abgeschwächt werden. Per Definition besitzt die laminare Grenzschicht im statistischem Mittel ein skaliertes Messsignal von 1. Dies kann beispielsweise anhand der Wahrscheinlichkeitsdichte vom skalierten Messsignal überprüft werden und Abweichungen können zur Korrektur der Skalierung genutzt werden (Schneider 1995). Schneider variierte die Transitionslage auf einer ebenen Platte durch das systematische Einbringen von zusätzlichen Störungen in die laminare Grenzschicht stromauf der eigentlichen Messposition. Ohne zusätzliche Störungen war die Grenzschicht am Messpunkt vollständig laminar und ermöglichte eine objektive Bestimmung des laminaren Referenzsignals.

In den Messungen dieser Arbeit gibt es keine vergleichbare Möglichkeit das laminare Referenzsignal objektiv zu bestimmen, sodass dieses aus dem intermittenten Messsignal extrahiert werden muss. Im Wavelet-Spektrum unterscheiden sich die Turbulenzflecken von der laminaren Grenzschicht durch ihr breitbandiges Spektrum. Dieses Unterschei- 

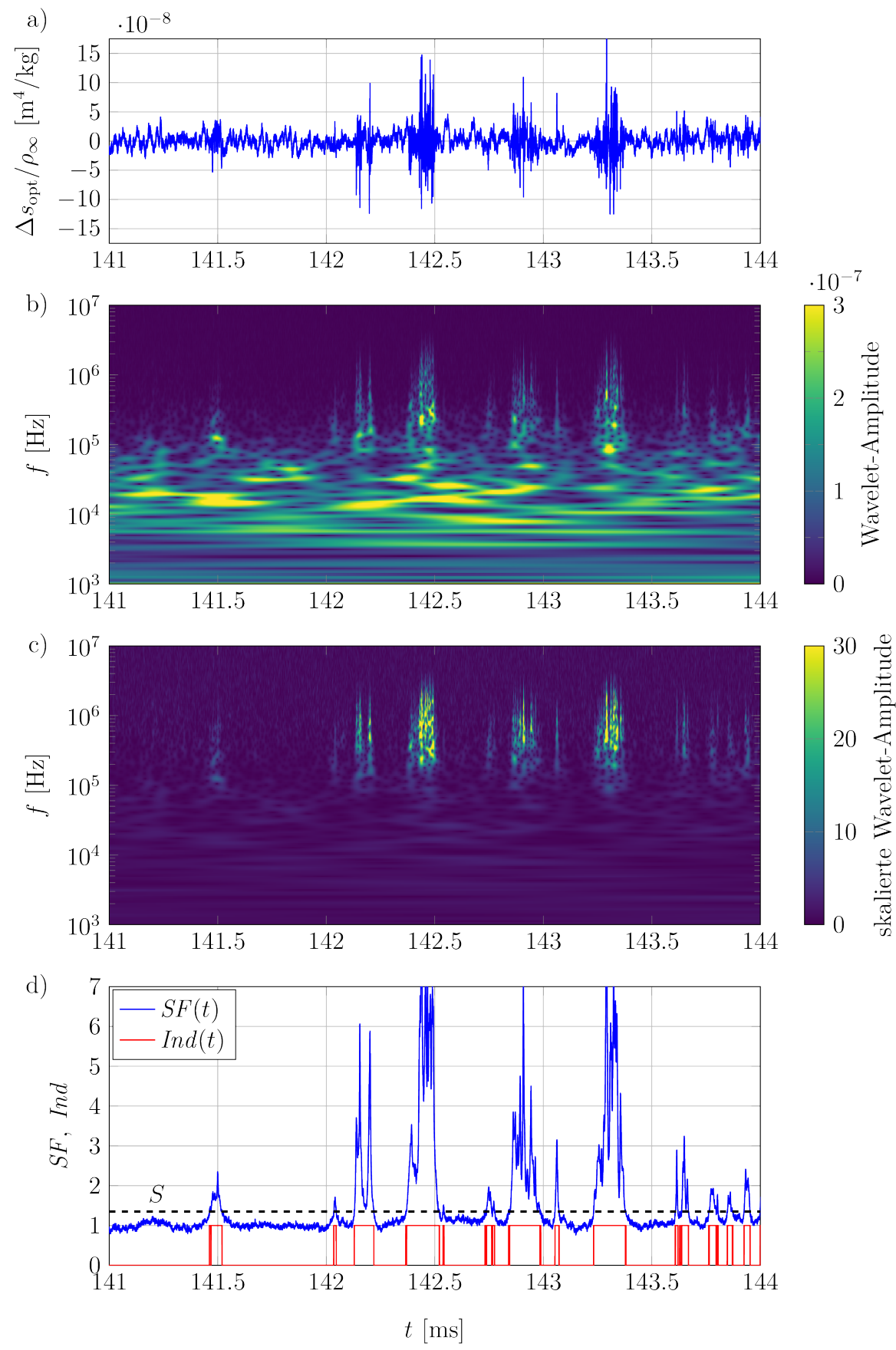

Abbildung 4.12: Darstellung der Zwischenschritte bei der Bestimmung von $\gamma$ mittels Detektion der Turbulenzflecken. a) zeigt den zeitlichen Verlauf des Messignals und b) die dazugehörige Wavelet-Transformation. Die Skalierung der Wavelet-Transformation (c) mit dem Spektrum der laminaren Grenzschicht erleichtert die Detektion der Turbulenzflecken gegenüber Störsignalen wie den Schwankungen in der Außenströmung. In d) sind die berechnete Selektionsfunktion $S F(t)$, der Schwellenwert $S$ und die daraus abgeleitete Indikatorfunktion $\operatorname{Ind}(t)$ dargestellt. 
dungsmerkmal wird für die Extraktion des laminaren Referenzsignals aufgegriffen. Hierfür wird an jedem Zeitpunkt die Wavelet-Amplitude über alle Frequenzen gemittelt. Ist die gemittelte Wavelet-Amplitude kleiner als ein gewisser Schwellenwert, so wird dieser Zeitpunkt als laminares Referenzsignal verwendet. Der Schwellenwert wird angepasst bis die Wahrscheinlichkeitsdichte der nachfolgend definierten Selektionsfunktion $S F$ einen Peak bei 1 besitzt. Das Wavelet-Frequenzspektrum der selektierten Messbereiche wird zeitlich gemittelt und ergibt ein statistisch representatives Wavelet-Frequenzspektrum von einer laminaren Grenzschicht. Ein Beispiel für dieses Spektrum ist in Abbildung 4.13 dargestellt und gleicht erwartungsgemäß dem Leistungsdichtespektrum einer laminaren Grenzschicht am Plattenmodell, das mittels FLDI in der RWG-Messstrecke gemessen wurde (vgl. Abb. 4.9p. Wird des Wavelet-Spektrum aus Abb. 4.12p mit dem repräsentativen Spektrum der laminaren Grenzschicht (Abb. 4.13) skaliert, so vereinfacht sich die computergestützte Detektion der turbulenten Zeitbereiche im Messsignal (vgl. Abb. 4.12 .). Um bei der Detektion der Turbulenzflecken das gesamte Frequenzspektrum zu berücksichtigen, wird die Selektionsfunktion $S F(t)$ an jedem Zeitpunkt aus dem Mittelwert über alle skalierten Wavelet-Frequenzen berechnet (vgl. Abb. 4.12 d).

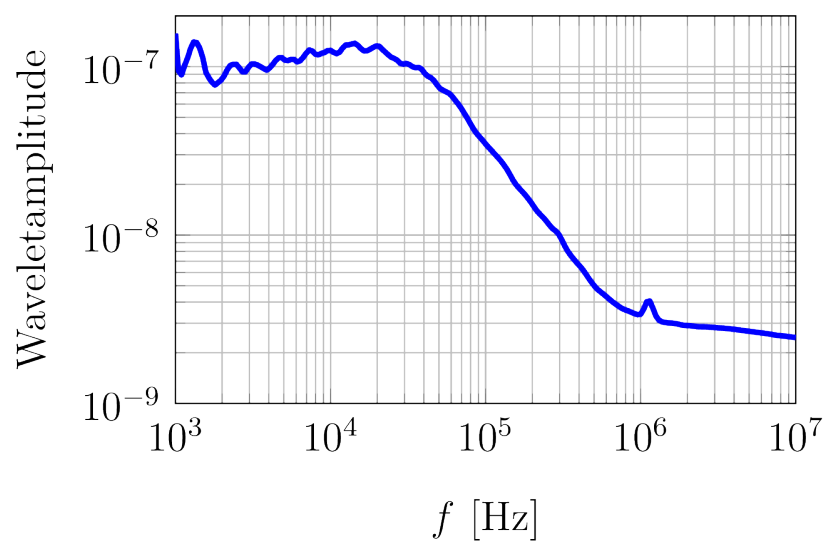

Abbildung 4.13: Beispiel für das gemittelte Wavelet-Spektrum einer laminaren Grenzschicht

Abbildung 4.14 zeigt die Wahrscheinlichkeitsdichteverteilung von $S F(t)$ bei verschiedenen Plattenlauflängen und verdeutlicht wie die Selektionsfunktion auf die einsetzende Transition reagiert. Wie bei Schneider (1995) gelingt es durch die Skalierung ein Peak in der Wahrscheinlichkeitsdichte bei 1 zu erzeugen, der als laminare Grenzschicht identifizierbar ist. Falls die Transition an einem Messpunkt soweit entwickelt ist, dass keine sinnvolle Auswahl an laminaren Messbereichen möglich ist, wird das laminare Referenzspektrum von der Messposition stromauf wiederverwendet. Die Histogramme in Abbildung 4.14 zeigen eine Verlagerung der Wahrscheinlichkeitsdichte, die im Zuge der laminar-turbulenten Transition und der damit einhergehenden Häufung von Turbulenzflecken stattfindet. Die Histogramme verdeutlichen somit, dass anhand der gewählten Selektionsfunktion eine Detektion der Turbulenzflecken möglich ist. Die Unterscheidung erfolgt mittels Schwellenwert. Der Schwellenwert wird wie in Veerasamy \& Atkin (2020) anhand einer Messposition festgelegt, die unmittelbar stromauf des Transitionsbeginns 


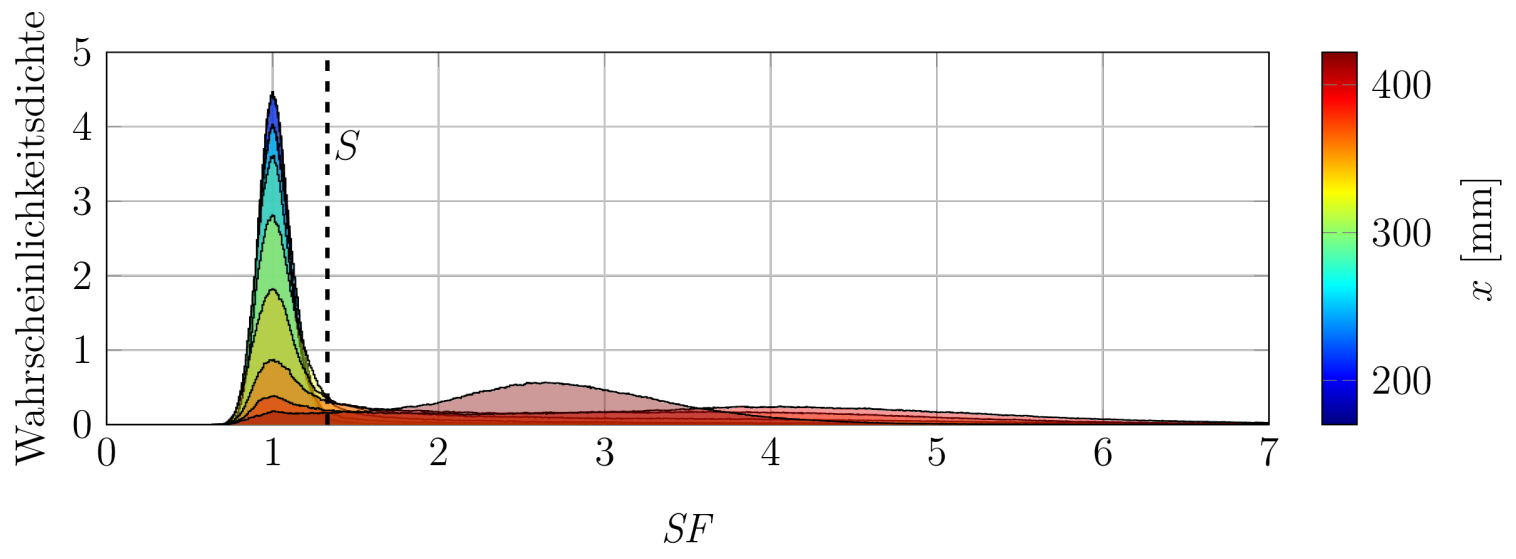

Abbildung 4.14: Wahrscheinlichkeitsdichteverteilung der Selektionsfunktion für $R e_{1}=$ $24 \cdot 10^{6} \mathrm{~m}^{-1}$ an diversen $x$-Positionen. Aufgrund der Transition findet eine Verlagerung der Wahrscheinlichkeitsdichte zu größeren SF-Werten statt.

liegt. Da für die jeweilige Anströmbedingung derselbe Schwellenwert an allen Messpositionen eingesetzt wird, verbessert sich dadurch die Vergleichbarkeit zwischen den Messpositionen. Wie Abbildung 4.15 zeigt, kann die Wahrscheinlichkeitsdichteverteilung der laminaren Grenzschicht adäquat durch eine logarithmische Normalverteilung beschrieben werden. Aus der logarithmischen Normalverteilung wurde der Schwellenwert anhand des 1\%o-Quantils gewählt. Das bedeutet in einer laminaren Grenzschicht werden nur $1 \%$ der Daten fälschlicherweise als turbulent eingestuft. In Abbilduung 4.12d ist der zeitliche Verlauf der Selektionsfunktion $S F(t)$ und die Indikatorfunktion $\operatorname{Ind}(t)$ dargestellt. Der zeitliche Verlauf gibt einen visuellen Eindruck von der Detektierung der Turbulenzflecken. Zur Bestimmung des Intermittenzwertes $\gamma$ wird die Indikatorfunktion über die Messzeit gemittelt.

\section{Intermittenzbestimmung anhand der $S t$-Verteilung}

Ein Modell um den mittleren quantitativen Wert der St-Zahl während der Transition abzuschätzen, besteht in der mit $\gamma$ gewichteten Linearkombination zweier ReferenzStanton-Zahlen für die laminare $\left(S t_{\mathrm{Tb}}\right)$ bzw. turbulente $\left(S t_{\mathrm{Te}}\right)$ Grenzschicht Dhawan \& Narasimha 1958, Narasimha 1985):

$$
S t=(1-\gamma) S t_{\mathrm{Tb}}+\gamma S t_{\mathrm{Te}}
$$

Umgeformt $\mathrm{zu}$

$$
\gamma=\frac{S t-S t_{\mathrm{Tb}}}{S t_{\mathrm{Te}}-S t_{\mathrm{Tb}}}
$$

kann mit diesem Ansatz die Verteilung des Intermittenzwertes auf Basis der experimentellen St-Messungen bestimmt werden (Benard et al. 2006, Schülein 2014). Das Problem 


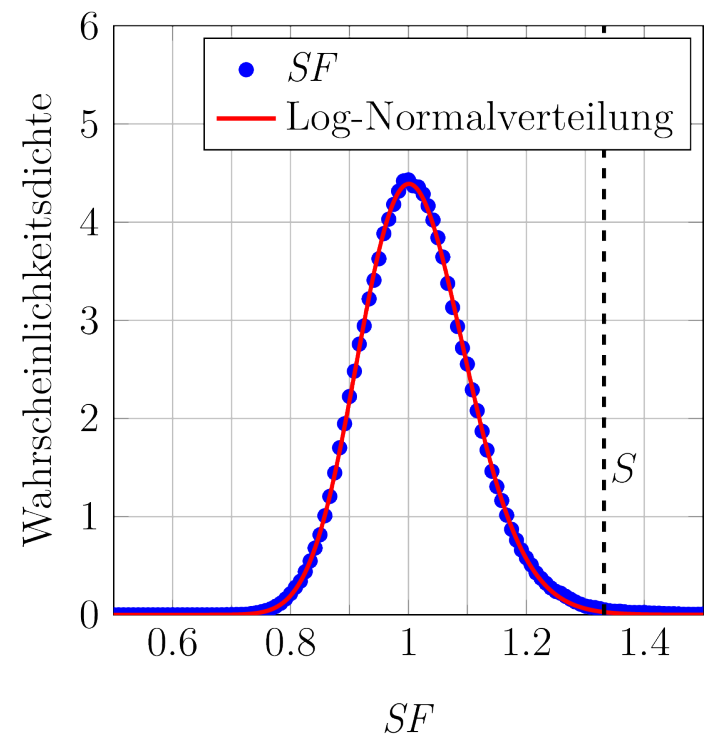

Abbildung 4.15: Wahrscheinlichkeitsdichte von SF für eine laminare Grenzschicht unmittelbar stromauf des Transitionsbeginns. Die Wahrscheinlichkeitsdichte kann durch eine logarithmische Normalverteilung nachgebildet werden.

bei dieser Variante besteht allerdings darin eine adäquate Beschreibung für die ortsabhängigen Referenzgrößen $S t_{\mathrm{Tb}}$ und $S t_{\mathrm{Te}}$ zu finden. Zur Bestimmung von $S t_{\mathrm{Te}}$ haben Schülein (2014), Lunte \& Schülein (2019) bei mehreren $R e_{1}$ die Stanton-Zahl am Transitionsende verwendet. Durch diese Punkte wurde der funktionale Zusammenhang

$$
S t_{\mathrm{Te}}=a_{\mathrm{Te}} \cdot R e_{x}^{b_{\mathrm{Te}}}
$$

gefittet. Die aktuellen Messdaten ließen nur für $R e_{1}=19 \cdot 10^{6} \mathrm{~m}^{-1}$ und $R e_{1}=$ $24 \cdot 10^{6} \mathrm{~m}^{-1}$ die Identifikation des Transitionsendes $\mathrm{zu}$, daher wird der Fitparameter $b_{\mathrm{Te}}$ in Anlehnung an das Ergebnis aus Lunte \& Schülein (2019) auf $b_{\mathrm{Te}}=-1 / 3$ gesetzt und nur der Parameter $a_{\mathrm{Te}}$ optimiert.

Die St-Verteilungen in Abbildung 4.3 zeigen, dass die Stanton-Zahl vor dem Transitionsbeginn etwas vom theoretischen Verlauf abweicht. Diese Unterschiede können durch die idealisierten Annahmen der theoretischen Herleitung entstehen oder hängen mit den internen Vorgängen der laminaren Grenzschicht vor Entstehung der Turbulenzflecken zusammen. In beiden Fällen sollte diese Abweichung bei der Intermittenzbestimmung berücksichtigt werden. Für jede $R e_{1}$ wird das Minimum im St-Verlauf als Transitionsbeginn interpretiert. Anhand der St-Werte beim Transitionsbeginn wird die Funktion

$$
S t_{\mathrm{Tb}}=a_{\mathrm{Tb}} \cdot R e_{x}^{b_{\mathrm{Tb}}}
$$

mit den Fitparametern $a_{\mathrm{Tb}}$ und $b_{\mathrm{Tb}}$ gefittet. Da der Transitionsbeginn bei sämtlichen $R e_{1}$ bestimmt werden konnte, werden diesmal beide Fitparameter verwendet. Mit diesem Vorgehen konnte aus den gemessenen St-Verteilungen eine Beschreibung für die 
Stanton-Zahl der beiden Referenzfälle abgeleitet werden, sodass der Intermittenzwert mit Gleichung 4.2 berechnet wird. Die Intermittenzwerte wurden stromauf von $R e_{\mathrm{Tb}}$ auf 0 und stromab von $R e_{\mathrm{Te}}$ auf 1 gesetzt. Abbildung 4.16 zeigt den Verlauf von $S t_{\mathrm{Tb}}$ und $S t_{\mathrm{Te}}$. Die Ergebnisse der Regression sowie die dafür verwendeten Werte sind in Tabelle A.6 und A.7 aufgelistet. Ob die gewählte Approximation für $S t_{\mathrm{Tb}}$ und $S t_{\mathrm{Te}}$ auch zu sinnvollen Intermittenzenwerten führt, wird im letzten Abschnitt dieses Kapitels diskutiert.

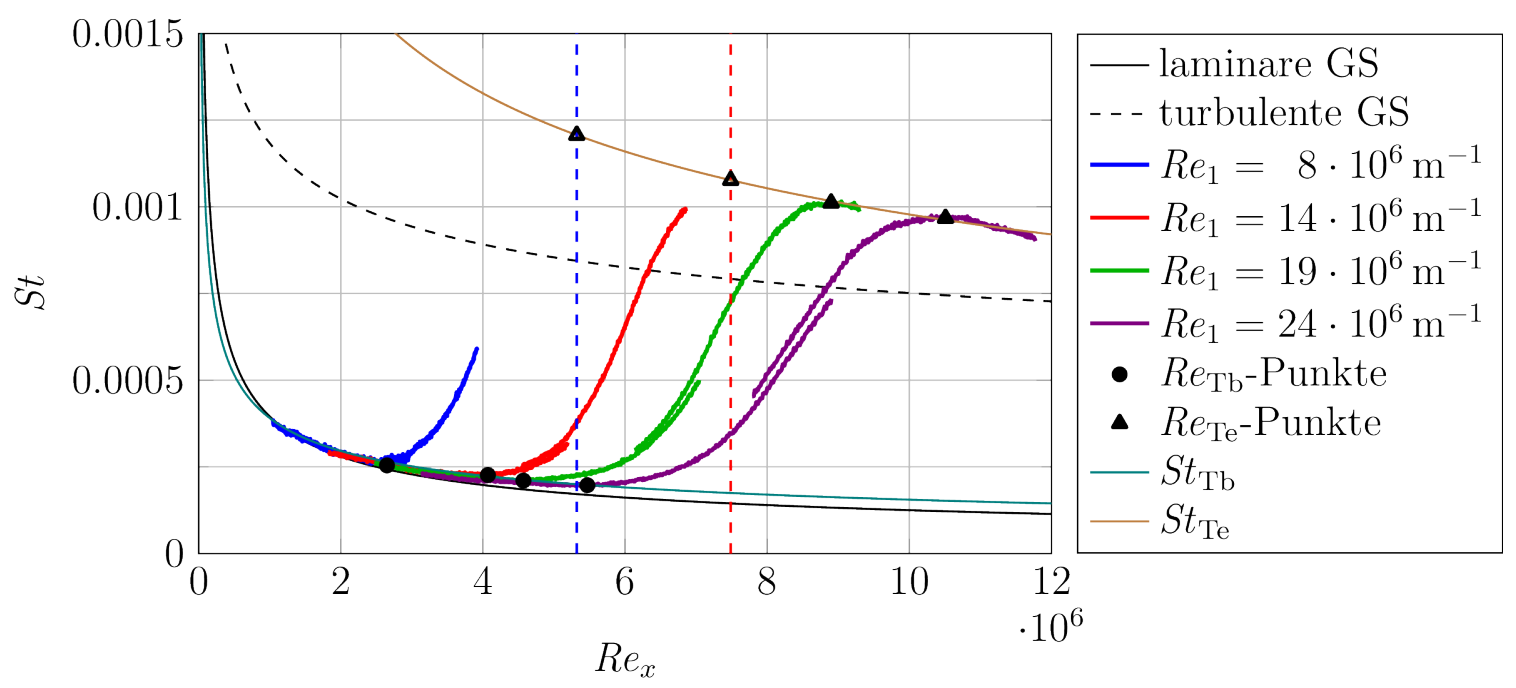

Abbildung 4.16: St-Verteilungen für diverse $R e_{1}$ an der Plattenposition A. Markiert sind die bestimmten $R e_{\mathrm{Tb}^{-}}$und $R e_{\mathrm{Te}_{\mathrm{T}}}$-Punkte sowie die daraus bestimmten Korrelationen $S t_{\mathrm{Tb}}$ und $S t_{\mathrm{Te}}$. Die gestrichelten vertikalen Linien zeigen die mit der Ross-Korrelation (Ross 1972) abgeschätzten $R e_{\mathrm{Te}}-$ Positionen für $R e_{1}=8 \cdot 10^{6} \mathrm{~m}^{-1}$ und $R e_{1}=14 \cdot 10^{6} \mathrm{~m}^{-1}$.

\section{Universelle Intermittenzverteilung}

Dhawan \& Narasimha (1958) zeigten auf Basis diverser experimenteller Intermittenzbestimmungen bei ebenen Platten, dass nach einer Skalierung der Plattenlauflänge ein universeller Verlauf für die Intermittenzwerte existiert. Eine Übersicht zu den unterschiedlichen funktionalen Approximationen bezüglich der universellen Intermittenzverteilung geben Narasimha \& Dey (1989). Die Approximation von Abu-Ghannam \& Shaw (1980)

$$
\begin{aligned}
\gamma & =1-\exp \left(-5 \zeta^{3}\right) & & \zeta \geq 0 \\
& =0 & & \zeta<0
\end{aligned}
$$

mit

$$
\zeta=\frac{R e_{x}-R e_{\mathrm{Tb}}}{R e_{\mathrm{Te}}-R e_{\mathrm{Tb}}}
$$


für eine feste $R e_{1}$ basiert auf den Positionen des Transitionsbeginns $\left(R e_{\mathrm{Tb}}\right)$ und des Transitionsendes $\left(R e_{\mathrm{Te}}\right)$. Bei $\zeta$ handelt es sich somit um eine lineare Skalierung der Plattenlauflänge auf Basis des Transitionsgebietes. Da sowohl $R e_{\mathrm{Tb}}$ als auch $R e_{\mathrm{Te}}$ aus den St-Verteilungen anhand der minimalen bzw. maximalen Wärmestromdichte bestimmt werden können, wird die Approximation von Abu-Ghannam \& Shaw (1980) in der aktuellen Arbeit verwendet. Für die untersuchten $R e_{1}$ sind in Tabelle A.6 und A.7 die verwendeten Werte von $R e_{\mathrm{Tb}}$ als auch $R e_{\mathrm{Te}}$ zur Berechnung von $\zeta$ aufgelistet. Abbildung 4.3 zeigt, dass für $R e_{1}=8 \cdot 10^{6} \mathrm{~m}^{-1}$ und $R e_{1}=14 \cdot 10^{6} \mathrm{~m}^{-1}$ keine experimentelle Bestimmung des Transitionsendes möglich ist. Vorangegangene Untersuchungen (Schülein 2014) am RWG bei $M a=6$ zeigten eine gute Übereinstimmung zwischen der experimentellen $R e_{1}$-Abhängigkeit von $R e_{\mathrm{Te}}$ und der Korrelation von Ross (1972). Aufgrund dieser Erfahrung wird die Ross-Korrelation $\left(R e_{\mathrm{Te}}=A \cdot R e_{1}^{0.61}\right)$ dazu verwendet, das Transitionsende bei den kleinen $R e_{1}$ abzuschätzen. Der Faktor $A$ wurde aus den $R e_{\mathrm{Te}^{-}}$ Werten von $R e_{1}=19 \cdot 10^{6} \mathrm{~m}^{-1}$ und $R e_{1}=24 \cdot 10^{6} \mathrm{~m}^{-1}$ bestimmt. Die ermittelten Werte für $R e_{\mathrm{Tb}}$ und $R e_{\mathrm{Te}}$ sind in Abbildung 4.16 auch visuell als Punkte dargestellt.

Ein weiteres Problem liegt bei der Bestimmung von $R e_{\mathrm{Tb}}$ an der Plattenposition $\mathrm{B}$ vor. Aufgrund der keilförmigen Störung der Stanton-Zahl (vgl. Kapitel 4.1.2) kann die $R e_{\mathrm{Tb}}$-Position nicht zweifelsfrei anhand des Minimums abgelesen werden. Aus diesem Grund wurde sich dafür entschieden die $R e_{\mathrm{Tb}}$-Stellen der Plattenposition A auch für die Plattenposition B zu verwenden.

\section{Analyse der Intermittenzergebnisse}

In den vorherigen Abschnitten wurden Methoden zur Intermittenzwertbestimmung auf Basis der in dieser Arbeit vorliegenden Messdaten eingeführt und erläutert, die in diesem Abschnitt miteinander analysiert werden sollen. Der Vergleich wird nur an der Plattenposition A durchgeführt, da hier die Ergebnisse der QIRT, PCB und FLDI Messtechniken zu den gleichen Strömungsbedingungen vorliegen. Abbildung 4.17 zeigt die ermittelten Intermittenzverteilungen bei den vier untersuchten $R e_{1}$.

Der Vergleich der Intermittenzverteilung offenbart, dass je nach Messtechnik die Transitionslage woanders detektiert wird. Bei den PCB-Daten befindet sich die Transitionslage am weitesten stromauf. Ein Grund dafür kann in der Montage der Sensoren liegen. Für die Sensoren mussten Löcher in die Einsatzplatte gebohrt werden und wegen der Schwingungentkopplung befindet sich eine $160 \mu \mathrm{m}$ breite Kavität um die einzelnen Sensoren (vgl. Abb. 3.10b). Die zusätzlichen Störungen, die durch den Spalt entstehen, können die messbare Transitionslage direkt beeinflussen. Ein weiteres Problem bezüglich der PCB-Sensoren besteht in der spannweitigen Verteilung der Messpositionen im Kombination mit dem realen spannweitig variablen Transitionsverlauf auf dem Modell. Als Konsequenz ergeben sich vergleichsweise sprunghafte Intermittenzwerte zwischen den einzelnen Messpunkten. Um den Effekt der spannweitig variablen Transitionslage zu visualisieren, wurde die St-Verteilung an mehreren spannweitigen Positionen ausgewertet und die Variation der Intermittenzverteilung als teiltransparente Fläche in Abb. 4.17 eingezeichnet. Bei $R e_{1}=24 \cdot 10^{6} \mathrm{~m}^{-1}$ und einem Intermittenzwert von 0.5 beträgt die Breite der teiltransparenten Fläche $21 \mathrm{~mm}$ in Strömungsrichtung. Die $\gamma_{S t}$-Verteilung 

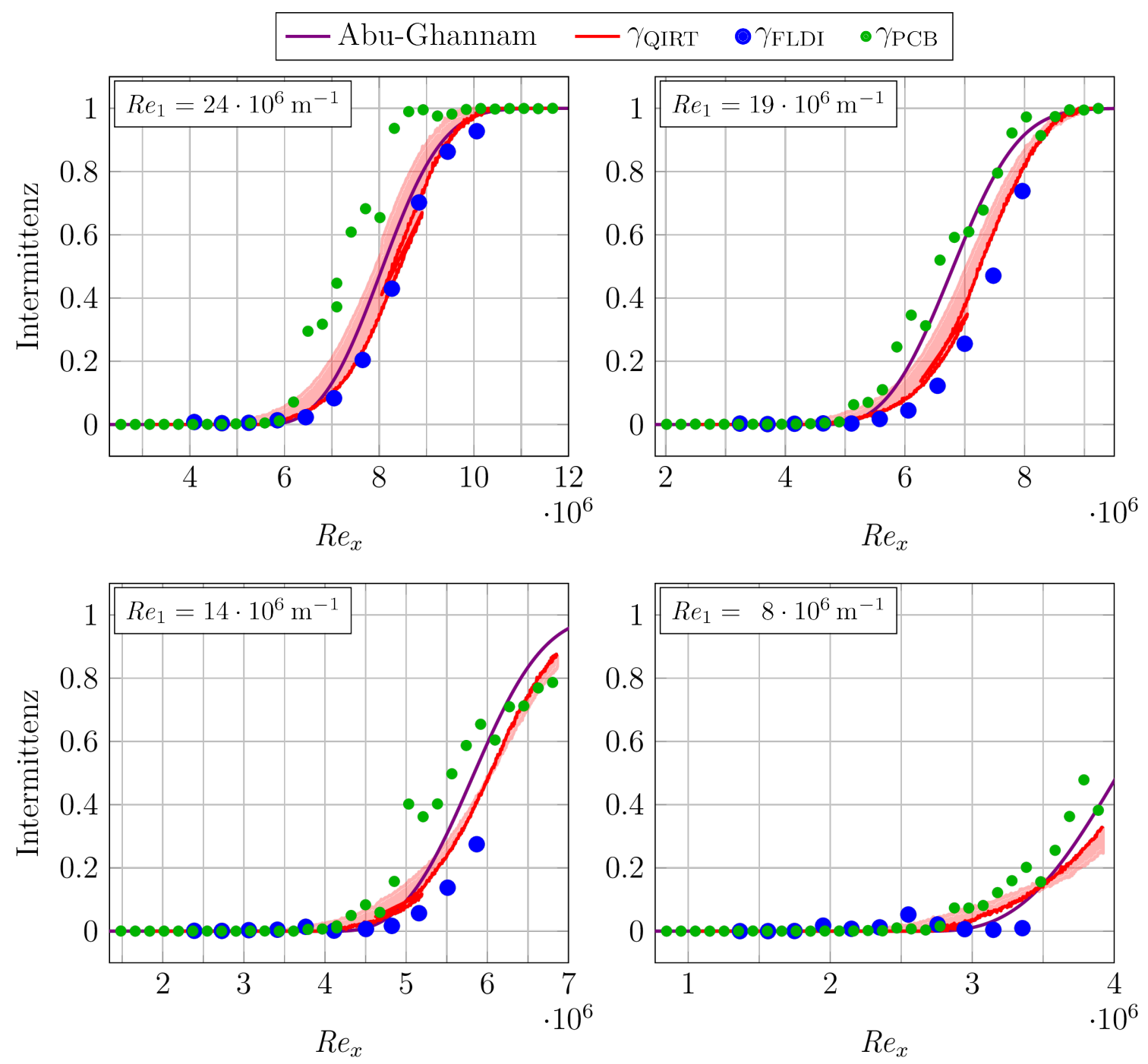

Abbildung 4.17: Vergleich der bestimmten Intermittenzverteilung anhand unterschiedlicher Verfahren und Strömungsgrößen für die untersuchten $R e_{1}$ an der Plattenposition A

entlang der Plattenmitte ist als undurchsichtige Linie eingezeichnet. Entlang der Plattenmitte befindet sich die Transitionslage weit stromab und stimmt für $R e_{1}=24 \cdot 10^{6} \mathrm{~m}^{-1}$ sehr gut mit $\gamma_{\mathrm{FLDI}}$ überein. Für die kleineren $R e_{1}$ verschlechtert sich die Übereinstimmung und nach den FLDI-Daten befindet sich die Transitionslage weiter stromab. Für die zwei stromabgelegensten Messpositionen von $\gamma_{\text {FLDI }}$ bei $R e_{1}=8 \cdot 10^{6} \mathrm{~m}^{-1}$ wurden die zugrundeliegenden Zeitsignale visuell auf Turbulenzflecken überprüft. Es konnten zwar vereinzelte Turbulenzflecken beobachtet werden, allerdings war deren zeitlicher Anteil an der Gesamtmesszeit gering, sodass für die überprüften Positionen ein Intermittenzwert nahe null plausibel ist und die Abweichung zu den anderen Methoden nicht auf einen fehlerhaften Auswertealgorithmus zurückzuführen ist.

Ein Grund für die Abweichungen zwischen den Messtechniken kann in der Verwen- 
dung der jeweils optimierten Einsatzplatten liegen. Die Einsatzplatten unterscheiden sich geringfügig in ihrer Oberflächenrauhigkeit $\left(R_{z}<1.5 \mu \mathrm{m}\right.$ für alle Einsätze) und in ihrer Temperaturerwärmung während der Messung. Zusätzlich musste bei jedem Wechsel der Einsatzplatte, der Übergang zwischen Basisplatte und Einsatz erneut bündig ausgerichtet werden. Durch diese unterschiedlichen Randbedingungen, kann auch eine strömungsmechanische Verschiebung der Transitionslage vorliegen.

Da die Transition nicht durch einen einzelnen skalaren Parameter beschrieben werden kann, existieren auch keine Referenzwerte für die Intermittenz, anhand derer die einzelnen Methoden objektiv bewertet werden können. Folglich bleibt nur die visuelle Überprüfung der Verfahren wie in Abb. 4.12d und der Vergleich der verschiedenen Methoden untereinander sowie mit dem universellen Intermittenzverlauf. Die Form der experimentell bestimmten Intermittenzverteilungen ist qualitativ vergleichbar zum universellen Verlauf. Im Detail stimmen die unterschiedlichen Methoden allerdings nicht überein. Der Vergleich verdeutlicht die Unsicherheiten bei der Berechnung des Intermittenzwertes.

Da der Intermittenzwert den zeitlichen Anteil an Turbulenzflecken innerhalb der Grenzschicht wiedergibt, stellt der Intermittenzwert eine quantitative Möglichkeit dar, den Transitionsfortschritt innerhalb des Transitionsgebietes zu quantifizieren. Außerhalb des Transitionsgebietes liefert der Intermittenzwert allerdings keine Aussage mehr über den Zustand der Grenzschicht. Die fehlende Einsortierung der laminaren bzw. turbulenten Grenzschicht ist der ausschlaggebende Grund, warum sich der Intermittenzwert zur Quantifizierung des Transitionsfortschrittes in Skalierungsgesetzen als ungeeignet erwies. Obwohl die skalierte Plattenlauflänge $\zeta$ (vgl. Gl. 4.7) am wenigsten die strömungsmechanischen Hintergründe während des Transitionsprozesses berücksichtigt, wird in den folgenden Auswertungen $\zeta$ für die Angabe des Transitionsfortschrittes verwendet, weil $\zeta$ auch außerhalb des Transitionsgebietes eine Aussage über den Strömungszustand liefert. Diese Eigenschaft ist insbesondere für die Beschreibung des Gebietes $\zeta<0$ von Vorteil, da sich die Dynamik der laminaren Grenzschicht mit der Lauflänge verändert. Bis zur Ausbildung der ersten Turbulenzflecken hat die ungestörte Grenzschicht bereits verschiedene Phasen der Transitionsentwicklung durchlaufen (vgl. Abb. 2.2). Abhängig von den internen Vorgängen verändert sich das Antwortverhalten der Grenzschicht bezüglich zusätzlich eingebrachter Störungen. Die durchgehende Einsortierung der Entwicklungsstufe relativ zur Lage der Transition ist der große Vorteil von $\zeta$ für die folgende Untersuchung der transitionellen SGWW.

\subsection{Stoß-Grenzschicht-Interaktion}

Nachdem im vorherigen Kapitel ausschließlich die ungestörte Umströmung einer ebenen Platte behandelt wurde, sollen nun die Auswirkungen von einfallenden Verdichtungsstößen auf die Strömung diskutiert werden. Zunächst wird anhand von Schattenaufnahmen die nicht-viskose Stoßauftreffposition ermittelt. Zusammen mit dem in Abschnitt 4.1.5 erarbeiteten Intermittenzwert und der skalierten Plattenlauflänge $\zeta$ ist es möglich den Grenzschichtzustand an der Stoßauftreffposition anzugeben. Anschließend wird in 
Abschnitt 4.2.2 die Auswirkung des Verdichtungsstoßes auf die Wärmestromverteilung diskutiert und der Einfluss von $R e_{1}$, Stoßintensität und Stoßauftreffposition erläutert. Anhand der Druck und Dichteschwankungen wird ein Einblick in die internen Vorgänge der Grenzschicht bei transitionellen SGWW gegeben. Abschließend werden die Erkenntnisse und Messdaten dazu verwendet, um Korrelationen für die Abschätzung charakteristischer Größen bei SGWW zu erarbeiten.

\subsubsection{Schattenaufnahmen}

Schattenaufnahmen der Strömung an der ebenen Platte mit montiertem Stoßgenerator sind exemplarisch in Abbildung 4.18 dargestellt. Die beiden Aufnahmen zeigen zwei Extremfälle der Parameterstudie bezüglich Stoßintensität, $R e_{1}$ und der vertikalen Position des Stoßgenerators (Abb. 4.18a $R e_{1}=8 \cdot 10^{6} \mathrm{~m}^{-1}, \alpha=2^{\circ}, 10 \mathrm{~mm}$ Offset; Abb. 4.18b. $R e_{1}=24 \cdot 10^{6} \mathrm{~m}^{-1}, \alpha=4^{\circ}, 0 \mathrm{~mm}$ Offset $)$.

Zunächst wird Abbildung 4.18a näher diskutiert. In der Aufnahme ist deutlich der Schatten des Stoßgenerators $(z>60 \mathrm{~mm})$ und der Halterung $(x>329 \mathrm{~mm})$ zu erkennen. Die Vorderkante des Stoßgenerators ist der Ursprung eines Verdichtungsstoßes, der die Stömung in diesem Fall um $2^{\circ}$ in Richtung ebene Platte umlenkt. An der Position $(x, z)=$ $(162 \mathrm{~mm}, 36 \mathrm{~mm})$ kommt es zu einer Stoß-Stoß-Interaktion mit dem Vorderkantenstoß der ebenen Platte. Aufgrund der unterschiedlichen Stoßintensität ändert sich der Winkel des einfallenden Stoßes nur geringfügig und trifft schließlich auf die ebene Platte, wo eine SGWW entsteht. Wegen des Druckanstiegs und der Stromaufwirkung nimmt die Grenzschichtdicke bereits vor der Auftreffstelle zu. Ob die Grenzschicht im dargestellten Fall ablöst, kann anhand der Schattenaufnahme nicht bestimmt werden. Allerdings ist eine Ablösung aufgrund der Ausdehnung der SGWW und der bei $R e_{1}=8 \cdot 10^{6} \mathrm{~m}^{-1}$ laminaren Grenzschicht wahrscheinlich. Aufgrund der kleinen $R e_{1}$ ist die Grenzschicht an der Unterseite des Stoßgenerators vollständig laminar.

In Abbildung $4.18 \mathrm{~b}$ ist eine Strömungssituation für $R e_{1}=24 \cdot 10^{6} \mathrm{~m}^{-1}$ und einem Stoßgenerator-Anstellwinkel von $4^{\circ}$ dargestellt. Da sich der Stoßgenerator nicht über die gesamte Breite der ebenen Platte erstreckt, kann im Schattenbild auch der Verlauf des Vorderkantenstoßes ohne Stoß-Stoß-Interaktion beobachtet werden. Erwartungsgemäß verläuft dieser oberhalb des abgelenkten Vorderkantenstoßes und ist deutlich kontrastärmer.

An der Unterseite des Stoßgenerators sind zwei weitere Stöße zu erkennen, die bei $x=172 \mathrm{~mm}$ und $x=192 \mathrm{~mm}$ ihren Ursprung haben. Der stromauf gelegene Stoß entsteht an der Kontaktstelle zwischen Stoßgenerator und dessen Verlängerung. Obwohl die Verlängerung bündig montiert wurde, ist das benutzte Schattenverfahren empfindlich genug um den induzierten schwachen Stoß zu visualisieren. Die Ursache des zweiten Stoßes ist unklar, da an dem Entstehungsort keine modellbezogene Kante existiert. Beide Verdichtungsstöße sind somit unbeabsichtigte schwache Störungen, die weiter stromab auf die Grenzschicht der ebene Platte treffen und z. B. auch in den gemessenen Wärmestromdichteverteilungen schwach detektierbar sind (siehe Unterkapitel 4.2.2).

Wie in Kapitel 3.3 beschrieben, wurde die Visualisierung der Verdichtungsstöße genutzt, um die nicht-viskose Auftreffposition zu ermitteln. Eine experimentelle Erfassung 


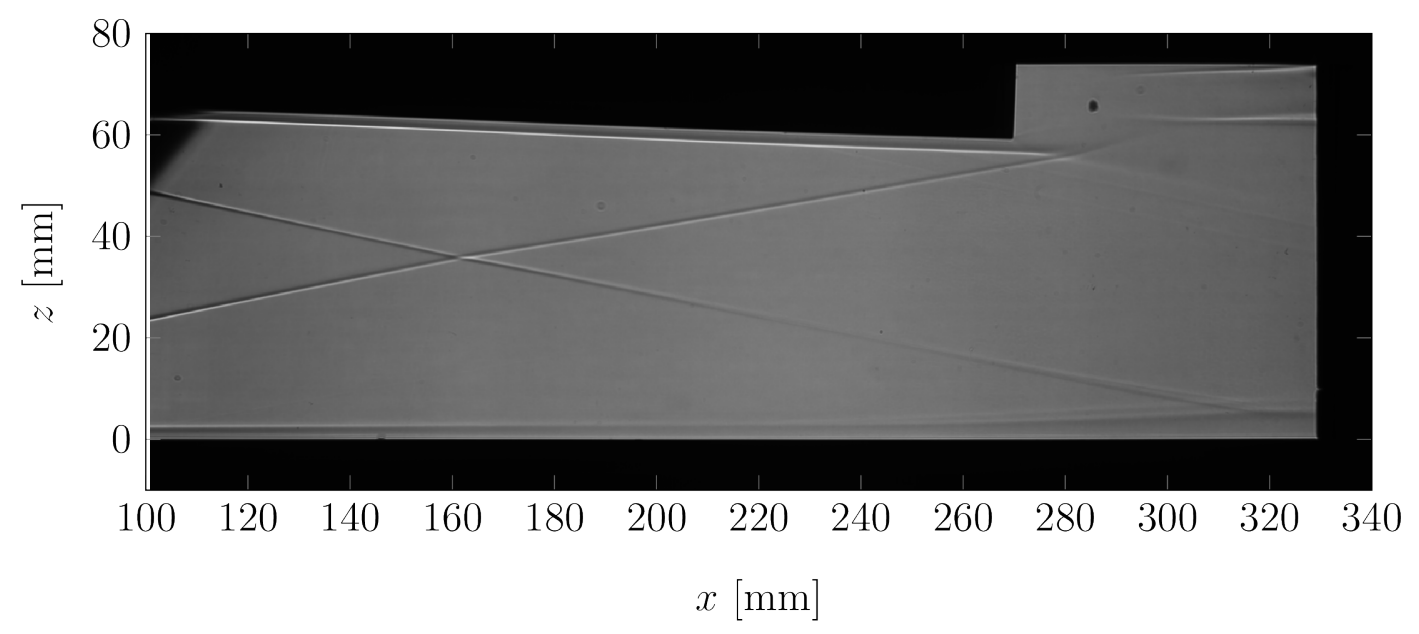

(a) $R e_{1}=8 \cdot 10^{6} \mathrm{~m}^{-1}, \alpha=2^{\circ}, 10 \mathrm{~mm}$ Offset

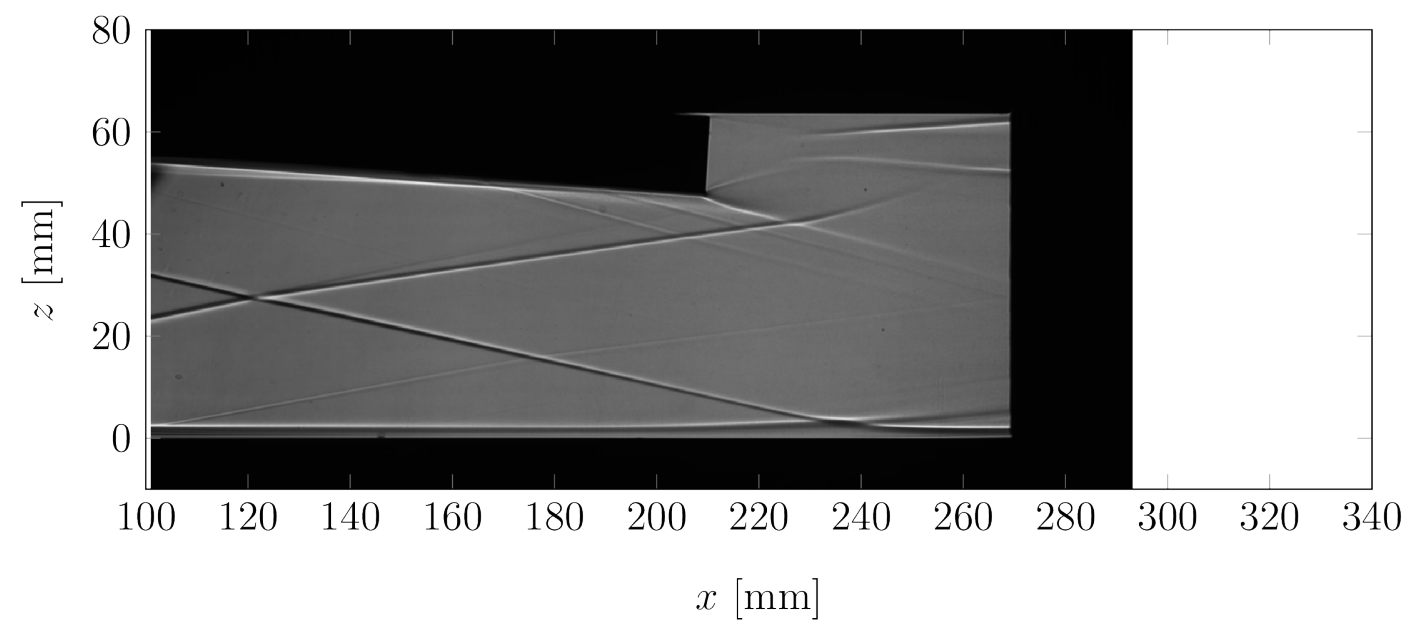

(b) $R e_{1}=24 \cdot 10^{6} \mathrm{~m}^{-1}, \alpha=4^{\circ}, 0 \mathrm{~mm}$ Offset

Abbildung 4.18: Schattenaufnahmen von der Umströmung der ebenen Platte mit montiertem Stoßgenerator.

der tatsächlichen Stoßauftreffposition ist unerlässlich, weil die Auswirkungen der Grenzschichtverdrängung am Stoßgenerator und der Stoß-Stoß-Wechselwirkung nicht ohne weiteres quantifiziert werden können. Die Ergebnisse sind in Tabelle 4.1 aufgelistet und wurden unter Vernachlässigung der räumlichen Verschiebung der Stoß-Stoß-Interaktion auf die einheitliche Stoßgeneratorposition Pos. I umgerechnet.

Anhand der Tabellenwerte lassen sich zwei Tendenzen erkennen. Zum einen verschiebt sich $x_{\text {imp }}$ stromab, wenn $R e_{1}$ zunimmt. Eine größere $R e_{1}$ bedeutet eine reduzierte Verdrängungsdicke (vgl. Gl. 2.13) am Stoßgenerator, wodurch der effektive Umlenkwinkel des Stoßgenerators abnimmt und der Stoßwinkel flacher wird. Zusätzlich ist der Stoßgenerator aufgrund der Grenzschicht kein idealer Keil, sondern ein gekrümmter Verdrängungskörper, in dessen Nasenbereich sich eine gekrümmte Stoßfront ausbildet. Der Über- 


\begin{tabular}{|c|c|c|c|c|c|c|}
\hline \multirow[b]{2}{*}{$R e_{1}\left[10^{6} \mathrm{~m}^{-1}\right]$} & \multicolumn{3}{|c|}{$\begin{array}{c}x_{\text {imp }}[\mathrm{mm}] \text { bei } \\
0 \mathrm{~mm} \text { Offset }\end{array}$} & \multicolumn{3}{|c|}{$\begin{array}{l}x_{\mathrm{imp}}[\mathrm{mm}] \text { bei } \\
10 \mathrm{~mm} \text { Offset }\end{array}$} \\
\hline & $2^{\circ}$ & $3^{\circ}$ & $4^{\circ}$ & $2^{\circ}$ & $3^{\circ}$ & $4^{\circ}$ \\
\hline 8 & 114.1 & 122.1 & 126.6 & 162.1 & 166.4 & 168.8 \\
\hline 14 & 116.6 & 121.8 & 125.7 & 163.9 & 166.7 & 168.3 \\
\hline 19 & 118.9 & 124.1 & 127.1 & 166.7 & 171.1 & 171.9 \\
\hline 24 & 120.0 & 124.5 & 127.4 & 167.5 & 172.2 & 172.8 \\
\hline
\end{tabular}

Tabelle 4.1: Nicht-viskose Stoßauftreffposition anhand der Schattenaufnahmen für die Stoßgeneratorposition Pos. I bei verschiedenen Anstellwinkeln

gang von der gekrümmten Stoßfront zum letztlichen Stoßwinkel hängt auch von $R e_{1}$ ab und kann vereinfacht als räumliche Verschiebung des schrägen Verdichtungsstoßes aufgefasst werden. Beide Effekte verschieben $x_{\text {imp }}$ bei zunehmender $R e_{1}$ stromabwärts. Die zweite Tendenz zeigt sich beim Vergleich der Anstellwinkel. Je kleiner der Anstellwinkel des Stoßgenerators, desto weiter stromauf befindet sich $x_{\text {imp }}$. Bei kleinen Anstellwinkeln ist der Beitrag der Verdrängungsdicke an der effektiven Strömungsumlenkung des Stoßgenerators größer, was schließlich die stromauf Verlagerung von $x_{\text {imp }}$ nach sich zieht. Zudem bestätigen die Daten in Tabelle 4.1, dass durch die vertikale Verschiebung des Stoßgenerators um $10 \mathrm{~mm}$ der Abstand zwischen Stoßgeneratorspitze und $x_{\text {imp }}$ um circa $45 \mathrm{~mm}$ größer wird. Dadurch können auch SGWW bei großen Plattenlauflängen untersucht werden, ohne dass eine Reflexion des Vorderkantenstoßes am Stoßgenerator das Messgebiet beeinflusst.

\section{Varianz der Stoßauftreffposition}

Die starken Kontraste in dem Fall von Abbildung 4.18b ermöglichen die Bestimmung der Stoßauftreffposition auch für die zugrundeliegenden Einzelbilder. Abbildung 4.19a zeigt $x_{\text {imp }}$ gegenüber den Stoßwinkel für die insgesamt 200 Kurzzeitaufnahmen. Zusätzlich wurde der Messzeitpunkt jeder Aufnahme farblich gekennzeichnet und das Ergebnis der gemittelten Schattenaufnahme als roter Punkt dargestellt. Ein direkter Zusammenhang zwischen der Verteilung der Datenpunkte und dem Messzeitpunkt ist nicht ohne weiteres erkennbar. Stattdessen existiert eine Korrelation zwischen dem Stoßwinkel und $x_{\text {imp }}$. Es wirkt, als wäre der Entstehungsort der Kopfwelle kaum von den Schwankungen in der Anströmung bzw. Grenzschicht beeinflusst. Stattdessen fluktuiert der Stoßwinkel und erzeugt den scheinbar linearen Zusammenhang zwischen $\beta$ und $x_{\text {imp }}$.

Abbildung 4.19b zeigt die gleichen Datenpunkte, allerdings liegt der Fokus dieser Darstellung auf der Evaluierung der Messgenauigkeit. Hierzu wurde das Bestimmtheitsmaß $R^{2}$ der linearen Regression farblich dargestellt und Fehlerbalken für beide Parameter eingetragen, die aus der Standardabweichung der Fit-Parameter folgen. Die $R^{2}$-Werte nahe 1 bestätigen, dass der Verlauf des schrägen Verdichtungsstoßes sinnvoll durch die lineare Regression wiedergegeben werden kann und rechtfertigen somit auch die Extrapolation 


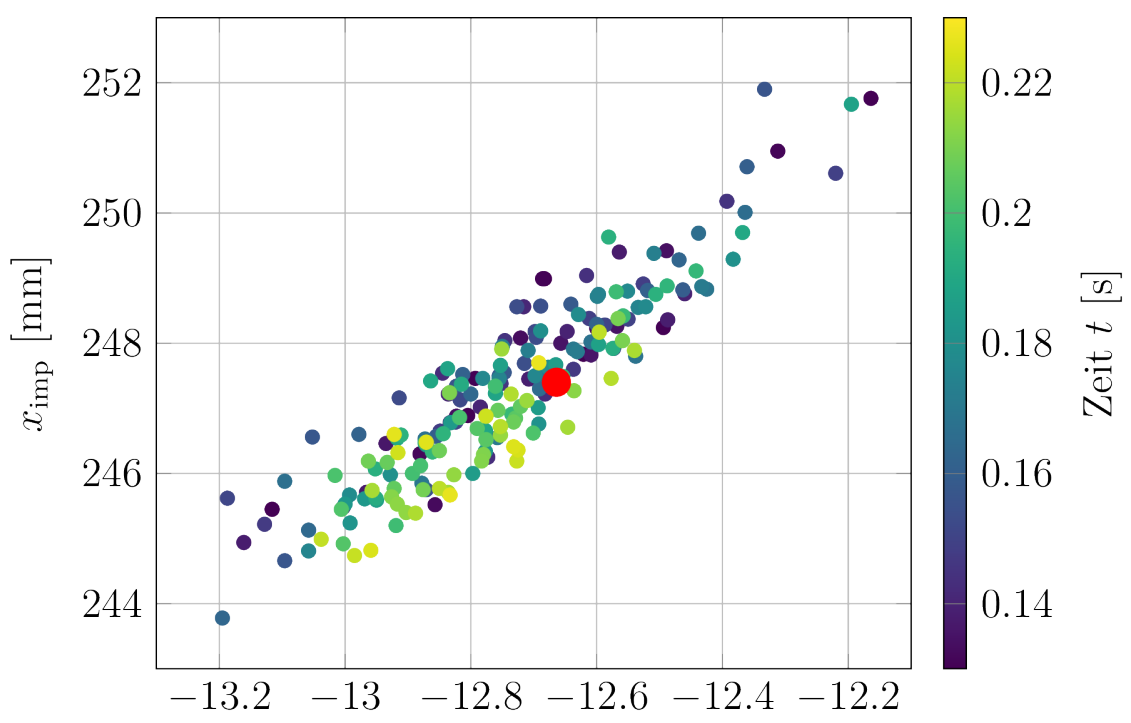

Stoßwinkel $\beta\left[^{\circ}\right]$

(a) Stoßauftreffposition und Stoßwinkel im zeitlichen Verlauf der Messung

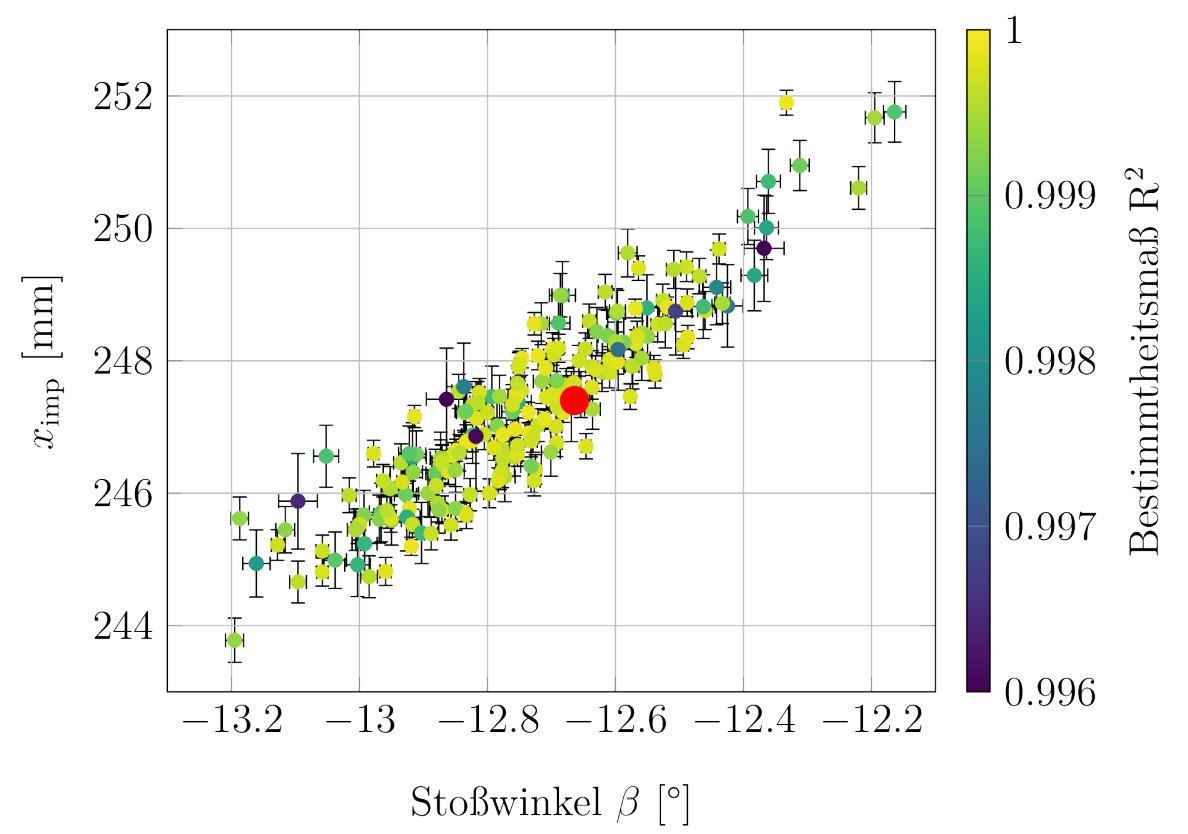

(b) Genauigkeit der linearen Regression

Abbildung 4.19: Bestimmung des einfallenden Verdichtungsstoßes mittels linearer Regression für 200 Einzelbilder

der Stoßauftreffposition. Die Fehlerbalken belegen, dass die Variation von $x_{\text {imp }}$ und dem Stoßwinkel $\beta$ durch Strömungseffekte größer ausfällt als die Messungenauigkeit.

Die Auswertung der Kurzzeitaufnahmen ermöglicht eine statistische Beschreibung der Parameter. Für die Stoßauftreffposition ergibt sich ein Mittelwert von $x_{\text {imp }}=$ 
$247.3 \mathrm{~mm}$ mit einer Standardabweichung von $\sigma_{x_{\mathrm{imp}}}=1.4 \mathrm{~mm}$ und für den Stoßwinkel $\beta=-12.74^{\circ} \pm 0.19^{\circ}$. Das Ergebnis der gemittelten Schattenaufnahme beträgt $x_{\text {imp }}=247.4 \mathrm{~mm}$ und $\beta=-12.66^{\circ}$. Beide Ergebnisse liegen innerhalb der Fehlerumgebung, sodass die Auswertung des gemittelten Bildes eine sinnvolle Repräsentation der gemittelten Stoßauftreffposition liefert. Der Wert $\sigma_{x_{\text {imp }}}=1.4 \mathrm{~mm}$ gibt einen Eindruck von der Variation der Stoßauftreffposition während der Messung, hat aber keine negativen Auswirkungen auf die weiterführenden Analysen. Bei schwächeren Stoßintensitäten reagiert $\beta$ stärker auf Störungen, sodass bei schwachen Stößen tendenziell größere Werte für $\sigma_{x_{\text {imp }}}$ zu erwarten sind.

\subsubsection{Stoßinduzierte Wärmestromdichte}

Ein Beispiel für die Stantonzahlverteilung auf der Modelloberfläche mit einer SGWW ist in Abb. 4.20a dargestellt. Wie die Abbildung belegt, sind aufgrund des zweidimensionalen Stoßgenerators die Effekte der SGWW spannweitig nahezu konstant. Auffällig sind die beiden Gebiete mit einer erhöhten Stanton-Zahl, die bei $(x, y) \approx(295 \mathrm{~mm}, \pm 75 \mathrm{~mm})$ beginnen und diagonal Richtung Mitte verlaufen. Die Ursache dieser Störungen liegt in der Halterung des Stoßgenerators. Die Verbindung der Halterung mit der ebenen Platte ist Ausgangspunkt eines neuen Stoßsystems, dass sich auch in das Messgebiet erstreckt. Wegen des festen geometrischen Abstandes zwischen Stoßgenerator-Spitze und dessen Halterung, verschiebt sich der parasitäre Effekt zusammen mit der Stoßauftreffposition. Somit bleibt in der Mitte des Messbereiches ein Streifen, der von den Störungen durch die Halterung unbeeinflusst ist und für die Analyse der SGWW genutzt werden kann. Die spannweitige Mittelung der St-Verteilung im Bereich $|y| \leq 25 \mathrm{~mm}$ erleichtert die Untersuchung der SGWW. Das Resultat der Mittelung ist in Abbildung 4.20 b zusammen mit dem St-Verlauf ohne Stoßinteraktion dargestellt. Durch den einfallenden und reflektierten Stoß wird das Fluid komprimiert, was wiederum zu einer Erhöhung der Wärmestromdichte führt. Die Auswirkung der Strömungskompression auf die StantonZahl lässt sich näherungsweise durch das Verhältnis

$$
\frac{\rho_{3} u_{3}}{\rho_{\infty} u_{\infty}}
$$

abschätzen. Die entsprechenden Kompressionsfaktoren für die untersuchten Ablenkwinkel können Tabelle A.2 entnommen werden. Durch Multiplikation des Kompressionsfaktors mit der St-Verteilung ohne Stoßinteraktion kann der Effekt der Kompression auf die Wärmestromdichte stromab der Stoßauftreffposition abgeschätzt werden. Die abgeschätzte Wärmestromdichte ist in Abbildung 4.20p in grau dargestellt und als „Kompression" betitelt.

Die St-Verteilung mit SGWW kann in vier Regionen unterteilt werden, die in Abb. $4.20 \mathrm{~b}$ durch gepunktete vertikale Hilfslinien getrennt sind. Der erste Bereich befindet sich stromauf des Interaktionsgebietes. Hier sollte die Wärmestromdichte mit der Grundströmung an der ebene Platte übereinstimmen. Im zweiten Bereich, stromauf der Stoßauftreffposition, verlangsamt sich die Strömung aufgrund des stoßinduzierten Druckanstiegs. Abhängig vom Zustand der Grenzschicht wird die Strömung verzögert oder löst ab, was 

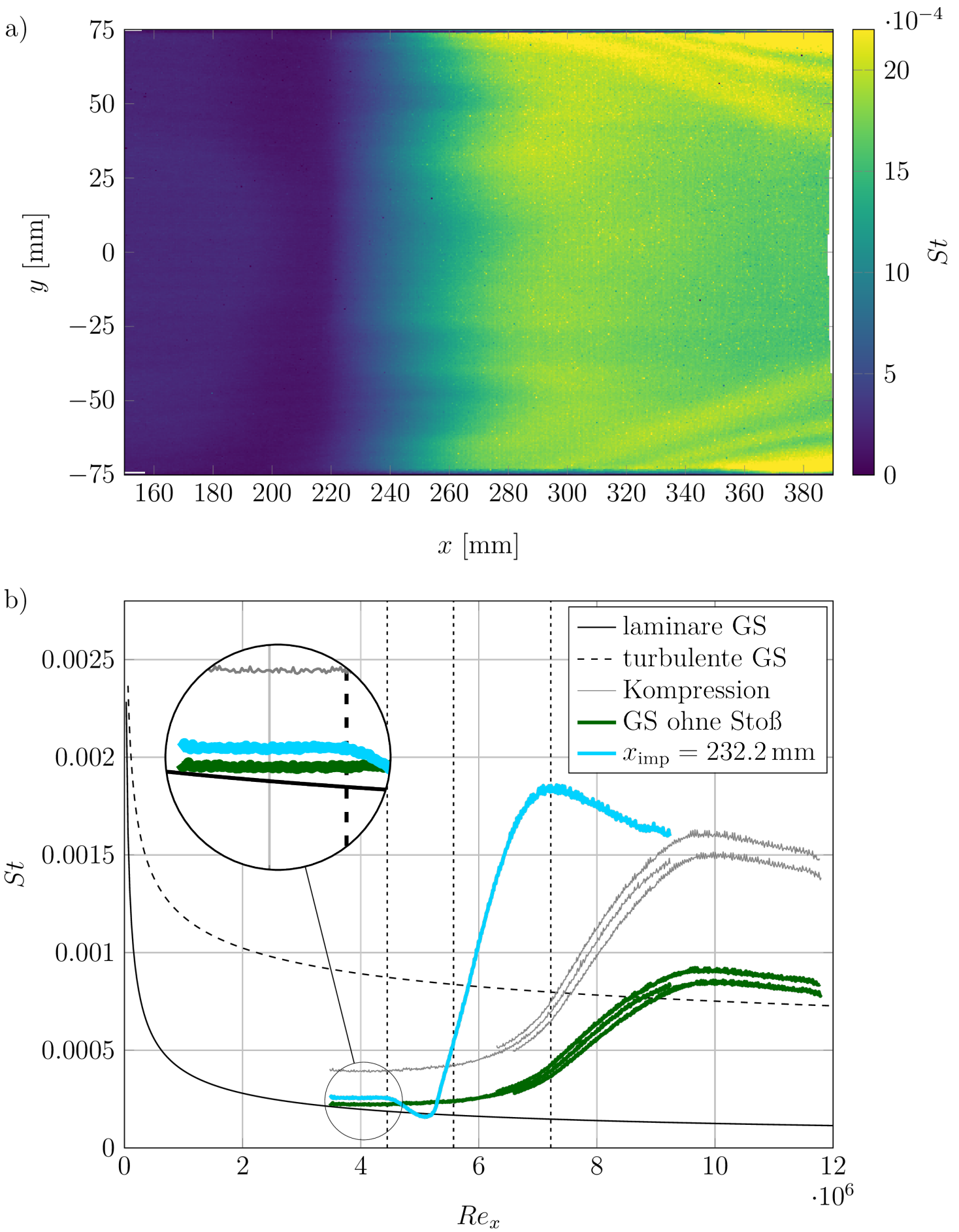

Abbildung 4.20: Beispiel für die St-Verteilung mit SGWW (a) und der spannweitig gemittelte $S t$-Verlauf im Vergleich zum ungestörten Fall (b) bei $R e_{1}=$ $24 \cdot 10^{6} \mathrm{~m}^{-1}$ 
beides die Wärmeübertragung reduziert. Andererseits erhöht der einfallende Stoß die Durchmischung, wodurch die Wärmestromdichte steigt. Abhängig vom jeweils lokal dominanten Effekt kann sowohl eine Reduzierung als auch eine Erhöhung von St vorliegen. Im dargestellten Fall wird eine Reduzierung der Stanton-Zahl beobachtet. Die Position, an der die Stanton-Zahl durch die SGWW beeinflusst wird, markiert die Stromaufwirkung der SGWW und wird mit $R e_{\mathrm{ui}}$ bezeichnet. Die Ausdehnung der Stromaufwirkung wird durch $\Delta R e_{\mathrm{ui}}=R e_{\mathrm{imp}}-R e_{\mathrm{ui}}$ mit $R e_{\mathrm{imp}}=x_{\mathrm{imp}} \cdot R e_{1}$ beschrieben. Die dritte Region beginnt stromab der Stoßauftreffposition und ist gekennzeichnet von einem rapiden Anstieg in der Stanton-Zahl. Die gesteigerte Wärmeübertragung entsteht aufgrund der Strömungskompression und der stoßinduzierten Durchmischung der Grenzschicht. Befindet sich die Grenzschicht an der Stoßauftreffposition in einem kritischen Zustand, kann der einfallende Stoß den Transitionsprozess einleiten bzw. beschleunigen. Die laminarturbulente Transition trägt zum rapiden Anstieg der Wärmestromdichte bei. Schließlich bildet sich eine maximale Stanton-Zahl $S t_{\max }$ aus. Stromab des Maximums beginnt der vierte Bereich, indem die stoßinduzierten Störungen abklingen und die Stanton-Zahl sich dem Niveau der Kompressionskurve annähert. Im dargestellten Verlauf beobachtet man bei $R e_{x} \approx 9 \cdot 10^{6}$ eine leichte Erhöhung der Stanton-Zahl, die auf die Verdichtungsstöße an der Unterseite des Stoßgenerators zurückzuführen sind (vgl. Kapitel 4.2.1). Unmittelbar stromab treffen auch die Expansionswellen von der Hinterkante des Stoßgenerators auf die ebene Platte und führen typischerweise zu einer Reduzierung der Stanton-Zahl. Die dargestellte St-Kurve endet aber bevor die Auswirkungen der Expansionswellen sichtbar werden.

Obwohl nach Theorie und Numerik die Stanton-Zahl im ersten Bereich identisch zum Referenzfall sein sollte, existiert in Abb. 4.20 b eine sichtbare Abweichung zwischen den Kurven bei etwa $R e_{x}=4 \cdot 10^{6}$. Eine Begutachtung aller SGWW lässt ein systematisches Verhalten erkennen, weshalb der Grund für die Abweichung näher untersucht wurde. Die Abweichung wächst sowohl mit $R e_{1}$ als auch mit der Stoßintensität und beträgt maximal $\Delta S t=3 \cdot 10^{-5}$. Es ergeben sich für laminare Grenzschichten die größten relativen Abweichungen. In einem ersten Schritt wurde die Kalibrierung der IR-Kamera überprüft und sowohl die Emissivität des Plexiglaseinsatzes als auch die Transmissivität des Germaniumfensters nachgemessen. Als Zweites wurde die Umrechnung der gemessenen Temperaturen zur Wärmestromdichte anhand der Cook und Feldermann Gleichung (Gl. 3.1) analysiert und mit numerischen Simulationen auf Basis der eindimensionalen Wärmegleichung verglichen. Hierbei wurde auch der Einfluss des Windkanalstarts auf die $\dot{q}_{\mathrm{w}}$-Ergebnisse während der stationären Strömung untersucht. Nachdem die Evaluierung des Auswerteprozesses keine Auffälligkeiten zeigte, wird vermutet, dass bei den Messungen mit Stoßgenerator zusätzliche Strahlung auf die Sensorfläche der IR-Kamera trifft. Die gemessene Strahlungsleistung setzt sich aus mehreren Komponenten entlang des Strahlengangs zusammen. Im IR-Bereich gibt jede Komponente mit ihrer Emissivität Strahlung ab. Zusammen mit der Transmission und Reflektivität aller Komponenten ergibt sich eine komplexe Zusammensetzung der gemessenen Strahlungsleistung. Durch die Montage des Stoßgenerators ändert sich die Situation in der Messstrecke, sodass sich die gemessene Strahlungsleistung beispielsweise über zusätzliche Reflexionen erhöhen kann, ohne dass eine reale Temperaturerhöhung am Messpunkt vorliegt. Diese 
Überlegung wurde in einer dedizierten Messkampagne untersucht, in der zunächst die Abweichung reproduziert wurde. Durch eine systematische Veränderung der Emissivität bzw. Reflektivität kritischer Komponenten wurde die Zusammensetzung der gemessenen Strahlungsleistung variiert, um so Rückschlüsse auf die Ursache der Abweichung zu ziehen. Allerdings ohne Erfolg, sodass die Abweichung der Stanton-Zahl stromauf des Interaktionsgebietes als systematischer Fehler in den Messdaten verbleibt.

Abbildung 4.21 zeigt den $S t$-Verlauf für verschiedene Stoßauftreffpositionen $x_{\text {imp }}$. Die Kurven sind anhand ihrer Stoßauftreffposition und der nebenstehenden Farbskala eingefärbt. Der Grenzschichtzustand bei $x_{\text {imp }}$ hat einen deutlichen Einfluss auf die gemessene Wärmestromentwicklung. Zum einen reduziert sich mit einsetzender Transition die Länge $L_{\mathrm{ui}}=\Delta R e_{\mathrm{ui}} / R e_{1}$, da sich der wandnahe Impuls der Strömung erhöht und dem Druckanstieg stärker entgegenwirkt. Zum anderen ist die erzeugte maximale StantonZahl $S t_{\max }$ am größten bei transitionellen SGWW. Ein weiterer Punkt ist die Steigung mit der die Stanton-Zahl ihr Maximum erreicht. Je weiter der Transitionsprozess bei der SGWW abgeschlossen ist, desto steiler entwickelt sich die Stanton-Zahl. Die Steigung kann auch als Maß interpretiert werden, wie schnell der Transitionsprozess aufgrund der stoßinduzierten Turbulenz beschleunigt wird. Ein flacher Anstieg bedeutet, dass die stoBinduzierte Turbulenz nicht für einen direkten Übergang zur turbulenten Grenzschicht ausreicht, stattdessen müssen die angeregten Störungen in der Grenzschicht wachsen, um die Transition zu vollenden.

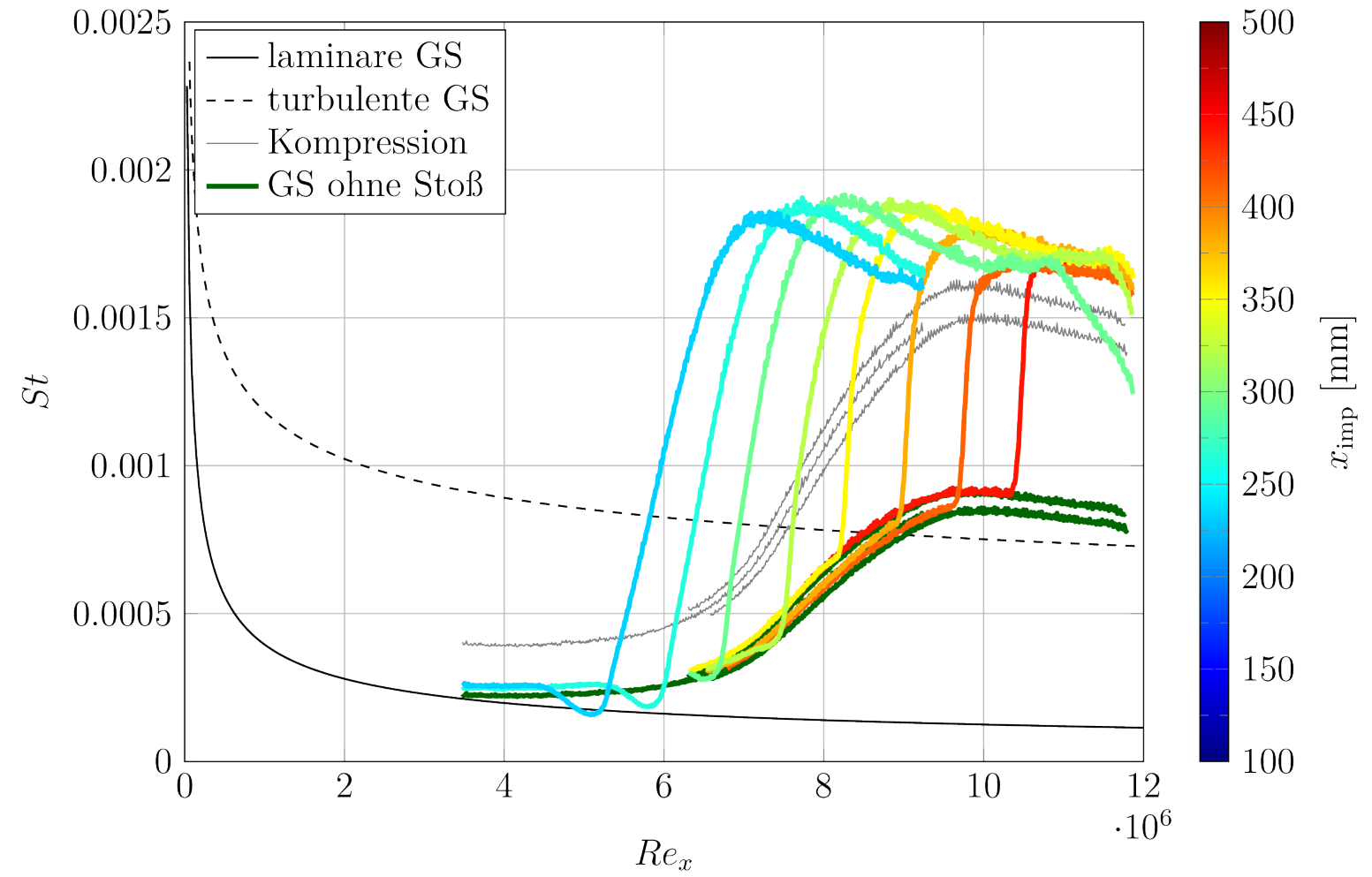

Abbildung 4.21: St-Verteilung mit SGWW bei $R e_{1}=24 \cdot 10^{6} \mathrm{~m}^{-1}$ für diverse $x_{\mathrm{imp}}$

Da der Grenzschichtzustand bei $x_{\text {imp }}$ die erzeugte Wärmestromdichte beeinflusst, ist 
auch ein Einfluss aufgrund der $R e_{1}$ zu erwarten. Anhand von Abb. 4.22 werden die Auswirkungen von $R e_{1}$ erläutert. Da die natürliche Transitionlage mit $R e_{1}$ variiert, ändert sich der Grenzschichtzustand für eine feste Stoßauftreffposition. Abb. 4.22 belegt somit, dass die höchsten $S t_{\max }$-Werte nicht an eine bestimmte Stoßauftreffposition geknüpft sind, sondern mit dem Transitionsfortschritt bei $x_{\text {imp }}$ korrelieren. Die Stromaufwirkung der SGWW verändert sich, da mit der Reduzierung von $R e_{1}$ die Verdrängungsdicke zunimmt (vgl. Gleichung 2.13). Bezüglich der St-Steigung stromab von $x_{\text {imp }}$ ist besonders der Fall für $R e_{1}=8 \cdot 10^{6} \mathrm{~m}^{-1}$ interessant. Für $x_{\mathrm{imp}}=226.4 \mathrm{~mm}$ ist eine Verzögerung in der St-Kurve sichtbar, die sogar zu einer Überlappung mit der nächsten stromab gemessenen St-Kurve führt. Im konkreten Fall findet die SGWW mit einer laminaren Grenzschicht statt und es scheint, dass die stoßinduzierte Turbulenz nicht ausreicht um einen direkten Transitionsbeginn einzuleiten. Erst nach einer gewissen Lauflänge scheinen die Störungen in der Grenzschicht ausreichend angewachsen zu sein, um den Transitionsprozess zu beginnen.

Abbildung 4.23 zeigt die Auswirkung der Stoßintensität auf die gemessenen StVerteilungen für eine feste Einheitsreynoldszahl von $R e_{1}=19 \cdot 10^{6} \mathrm{~m}^{-1}$. Durch eine größere Stoßintensität wird das Fluid stärker komprimiert und der Druckanstieg steigt. Im subsonischen Bereich der Grenzschicht verstärkt sich mit dem Druckanstieg die Verzögerung der Strömung, wodurch die Stromaufwirkung zunimmt. Durch eine stärkere Kompression des Fluids erhöhen sich auch die stoßinduzierten Wärmestromdichten und es wird mehr Turbulenz in die Grenzschicht eingebracht. Als Folge wird $S t_{\max }$ nach einer kürzeren Lauflänge erreicht. Die Stoßintensität beeinflusst auch, welche $x_{\text {imp }}$-Position den größten $S t_{\max }$-Wert erzeugt.

Bereits Abbildung 4.22c zeigte, dass bei einer unzureichenden Stoßintensität die eingebrachten Störungen gegebenenfalls nicht ausreichen, um den Transitionsprozess unmittelbar einzuleiten. Dieser Effekt ist bei einer geeigneten Kombination aus Stoßintensität und Grenzschichtzustand noch deutlicher ausgeprägt und in Abbildung 4.24 bei den Bedingungen $R e_{1}=8 \cdot 10^{6} \mathrm{~m}^{-1}, p_{3} / p_{1}=1.76$ dargestellt. Insbesondere für $x_{\mathrm{imp}}=144.1 \mathrm{~mm}$ scheint die Strömung auch stromab der Stoßauftreffposition zunächst laminar zu bleiben und die gemessene Stanton-Zahl liegt nur knapp oberhalb des reinen Kompressionseffektes. Erst nach einer gewissen Lauflänge scheint der laminar-turbulente Umschlag einzusetzen.

Abbildung 4.25a zeigt die gemessene St-Verteilung einer laminaren SGWW mit einer Stoßintensität von $p_{3} / p_{1}=2.95$ bei $R e_{1}=8 \cdot 10^{6} \mathrm{~m}^{-1}$. Unter diesen Bedingungen sind stromab der Stoßauftreffposition spannweitig periodische Variationen der Wärmestromdichte sichtbar. Vergleichbare spannweitige Effekte wurden bei SGWW auch in anderen Arbeiten (Ginoux 1969, Simeonides et al. 1994, Currao et al. 2020) beobachtet und auf Görtler-Wirbel zurückgeführt. Im klassischen Fall entstehen die Görtler-Wirbel an konkav gekrümmten Wänden (vgl. Kapitel 2.1.3). Bei SGWW führt die Trennlinie der Ablöseblase stromauf der Wiederanlegung allerdings zu einem vergleichbaren konkaven Stromlinienverlauf. Ginoux (1969) zeigte, dass initiale Instabilitäten, wie sie durch Unregelmäßigkeiten an der Vorderkante entstehen, innerhalb der abgelösten Scherschicht umstrukturiert werden und sich die gegenläufig rotierenden Wirbel ausbilden. Die Krümmung der konkaven Stromlinien beeinflusst die Intensität der Görtler-Wirbel, weshalb 

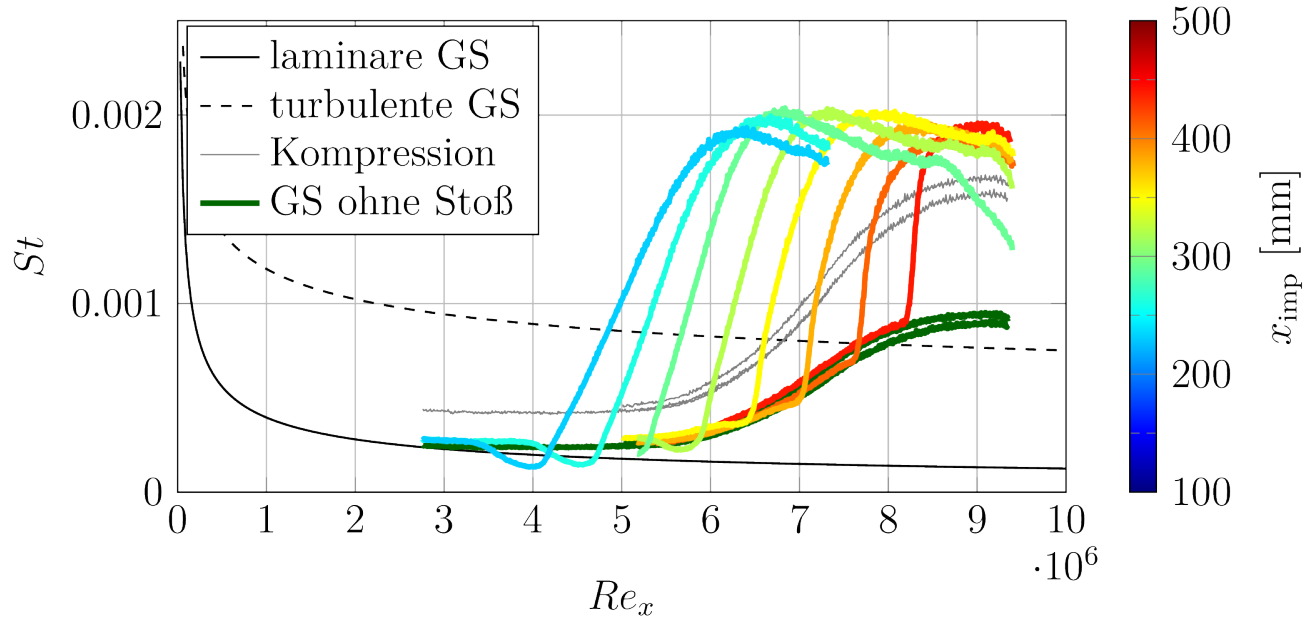

(a) $R e_{1}=19 \cdot 10^{6} \mathrm{~m}^{-1}$

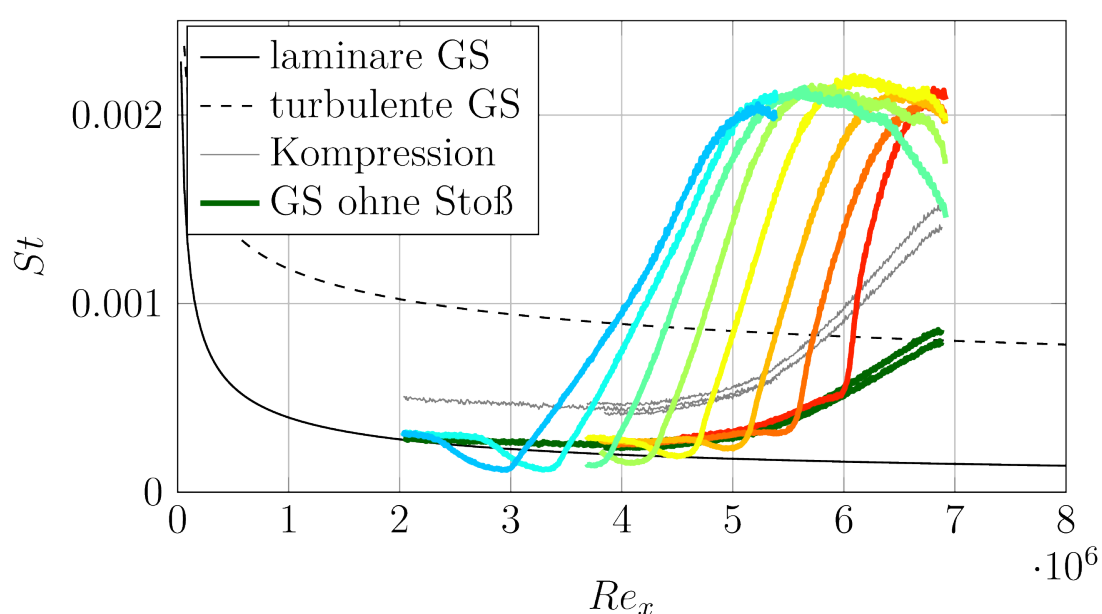

500

400

$300 \frac{\text { छ }}{\text { 当 }}$

200

(b) $R e_{1}=14 \cdot 10^{6} \mathrm{~m}^{-1}$

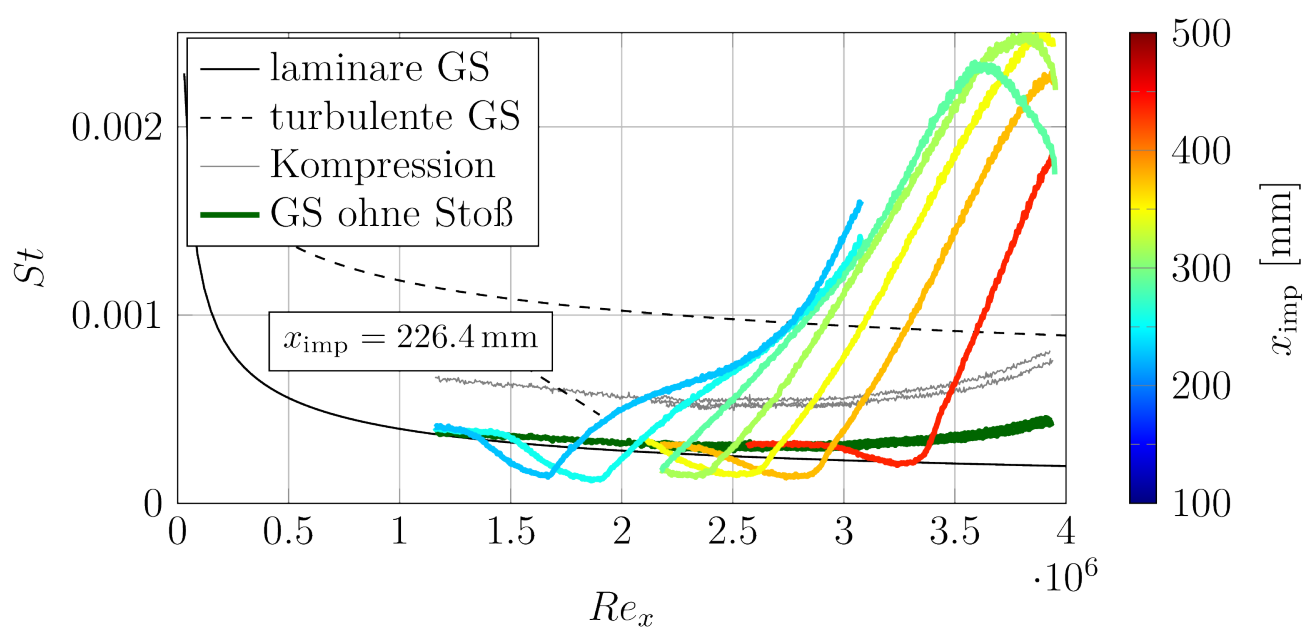

(c) $R e_{1}=8 \cdot 10^{6} \mathrm{~m}^{-1}$

Abbildung 4.22: St-Verteilungen bei der Stoßintensität $p_{3} / p_{1}=2.29$ für diverse $R e_{1}$ 


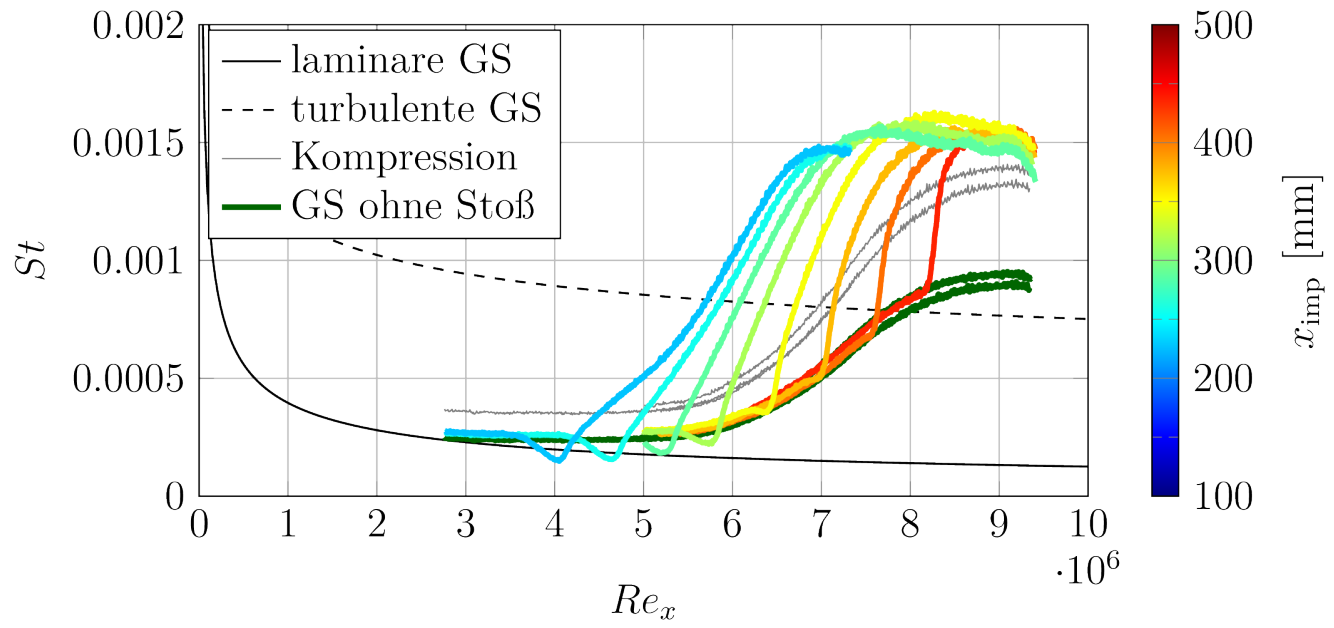

(a) $p_{3} / p_{1}=1.76$

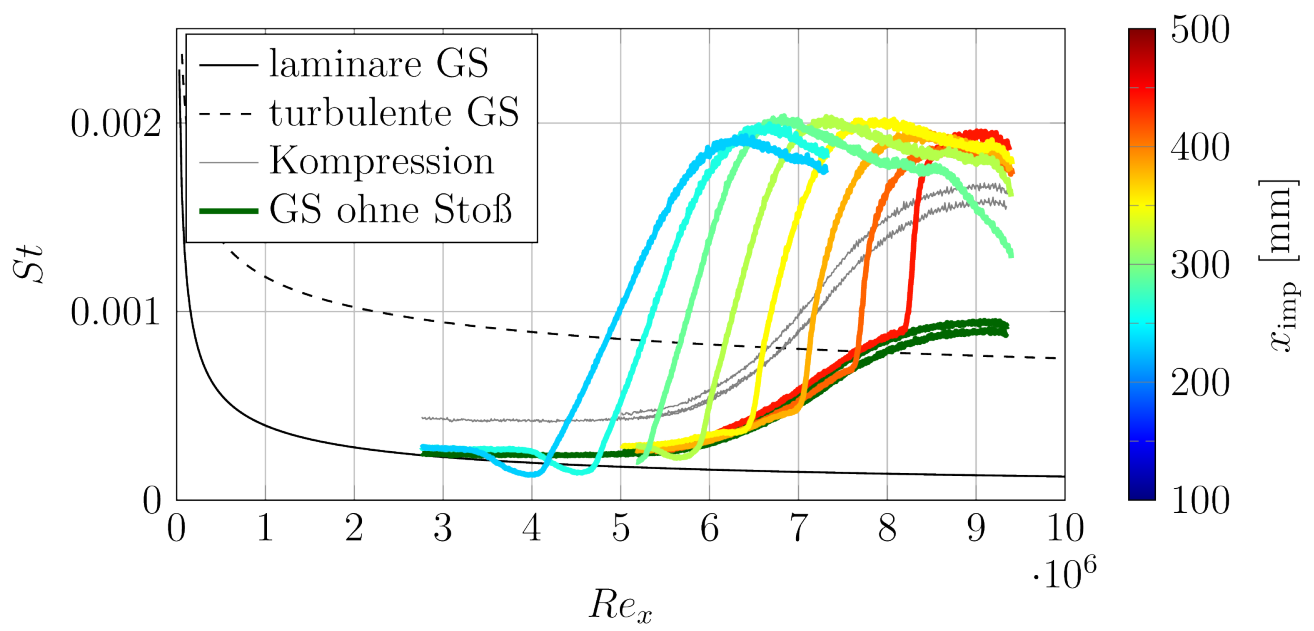

(b) $p_{3} / p_{1}=2.29$

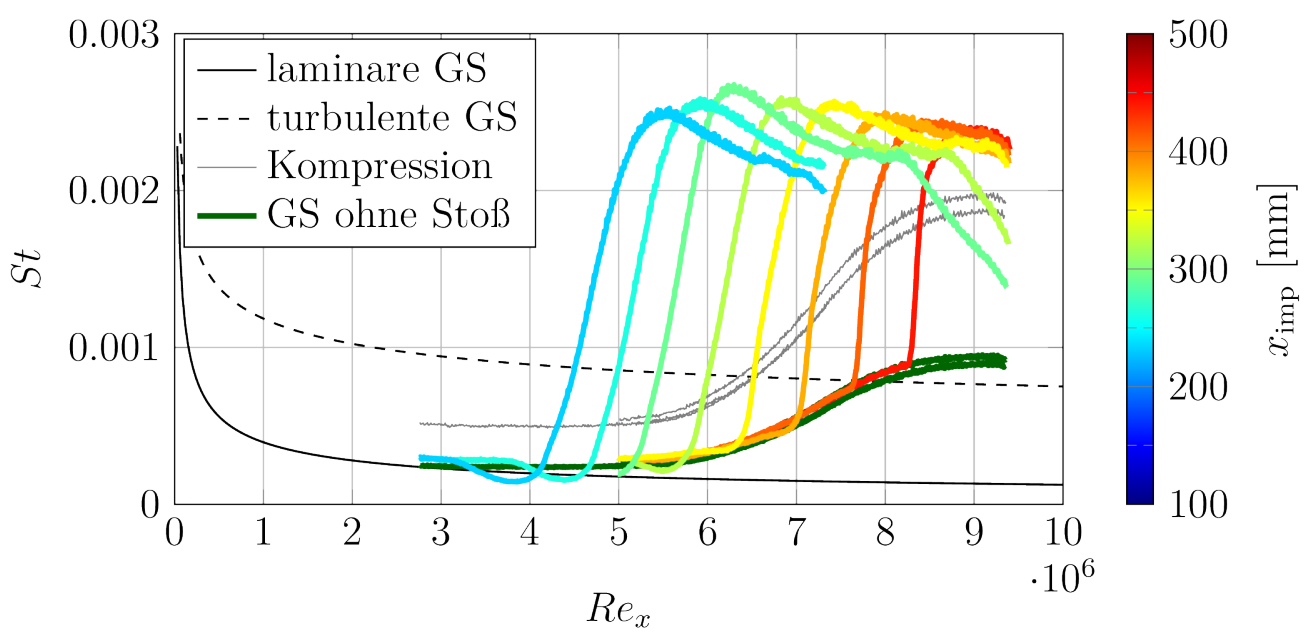

(c) $p_{3} / p_{1}=2.95$

Abbildung 4.23: St-Verläufe bei $R e_{1}=19 \cdot 10^{6} \mathrm{~m}^{-1}$ und diversen Stoßintensitäten 


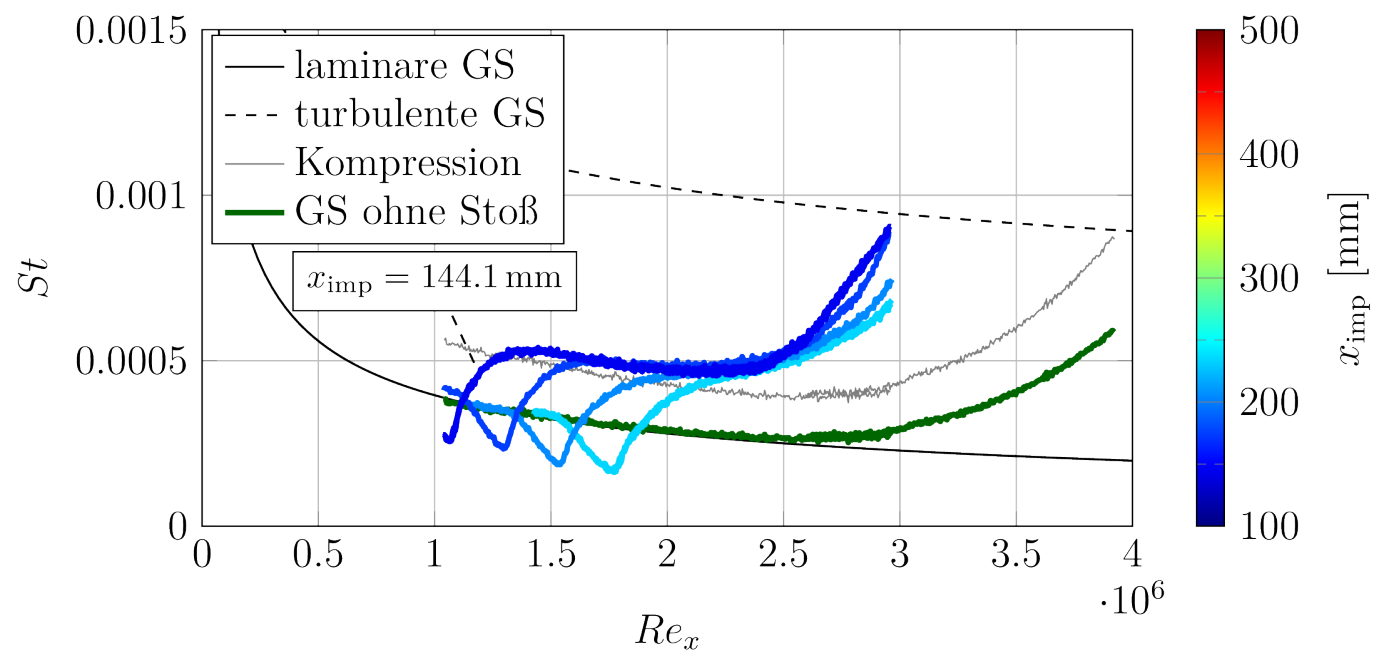

Abbildung 4.24: St-Verläufe bei $R e_{1}=8 \cdot 10^{6} \mathrm{~m}^{-1}$ und $p_{3} / p_{1}=1.76$ für diverse $x_{\text {imp }}$. Die Kurven zeigen, dass stromab von $x_{\text {imp }}$ die Transition nicht unmittelbar beginnt.

nur bei bestimmten Fällen der Parameterstudie die Längsstreifen in der St-Verteilung sichtbar sind. Die spannweitige Verteilung von $S t$ wurde entlang der Strömungrichtung über den Bereich $x=300 \mathrm{~mm} \pm 5 \mathrm{~mm}$ gemittelt und in Abbildung 4.25b dargestellt. Anhand der Maxima wurde die Wellenlänge zu $\lambda=8.4 \mathrm{~mm}$ bestimmt. Wird die Wellenlänge mit der laminaren Grenzschichtdicke der ebenen Platte an der Stoßauftreffposition $(\delta=3.71 \mathrm{~mm})$ skaliert, so beträgt $\lambda / \delta=2.3$ und liegt innerhalb des Wertebereiches von 1-4, welcher für die skalierten Wellenlängen von Görtler-Wirbeln in anderen Arbeiten gefunden wurde (Currao et al. 2020). 

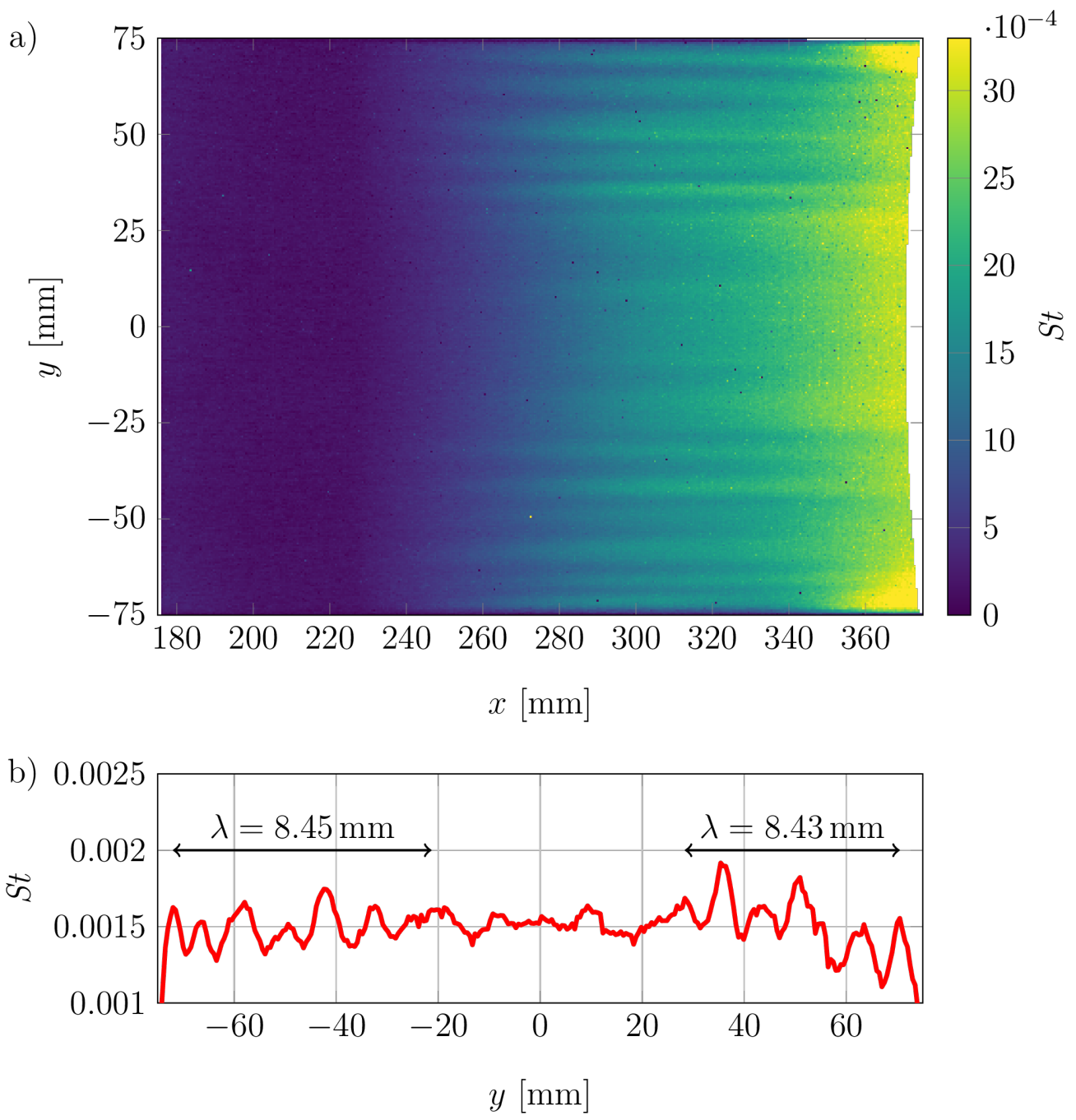

Abbildung 4.25: Die St-Verteilung (a) bei $p_{3} / p_{1}=2.95$ und $R e_{1}=8 \cdot 10^{6} \mathrm{~m}^{-1}$ zeigt spannweitige Variationen stromab der Stroßauftreffposition, die auf Görtler-Wirbel zurückgeführt werden. Anhand des spannweitigen StVerlaufs (b) bei $x=300 \mathrm{~mm} \pm 5 \mathrm{~mm}$ wurde die Wellenlänge der Wirbel bestimmt.

\subsubsection{Intermittenzverlauf bei SGWW}

Die FLDI-Technik und die PCB-Sensoren wurden eingesetzt um die Dichte- bzw. Druckschwankungen der Grenzschicht bei einer SGWW an mehreren Positionen entlang der Plattenlauflänge zu untersuchen. Aus den Zeitschrieben wurde der Intermittenzwert bestimmt, wobei das Verfahren aus Kapitel 4.1.5 zur Detektion von Turbulenzflecken eingesetzt wurde. Das Ergebnis dieser Auswertung ist in Abbildungen 4.26 und 4.27 für vier verschiedene $R e_{1}$ dargestellt (Abb. 4.26: $R e_{1}=8 \cdot 10^{6} \mathrm{~m}^{-1}$ und $R e_{1}=14 \cdot 10^{6} \mathrm{~m}^{-1}$; Abb. 4.27: $R e_{1}=19 \cdot 10^{6} \mathrm{~m}^{-1}$ und $R e_{1}=24 \cdot 10^{6} \mathrm{~m}^{-1}$ ).

In der oberen Zeile ist der St-Verlauf der untersuchten SGWW dargestellt und dient 

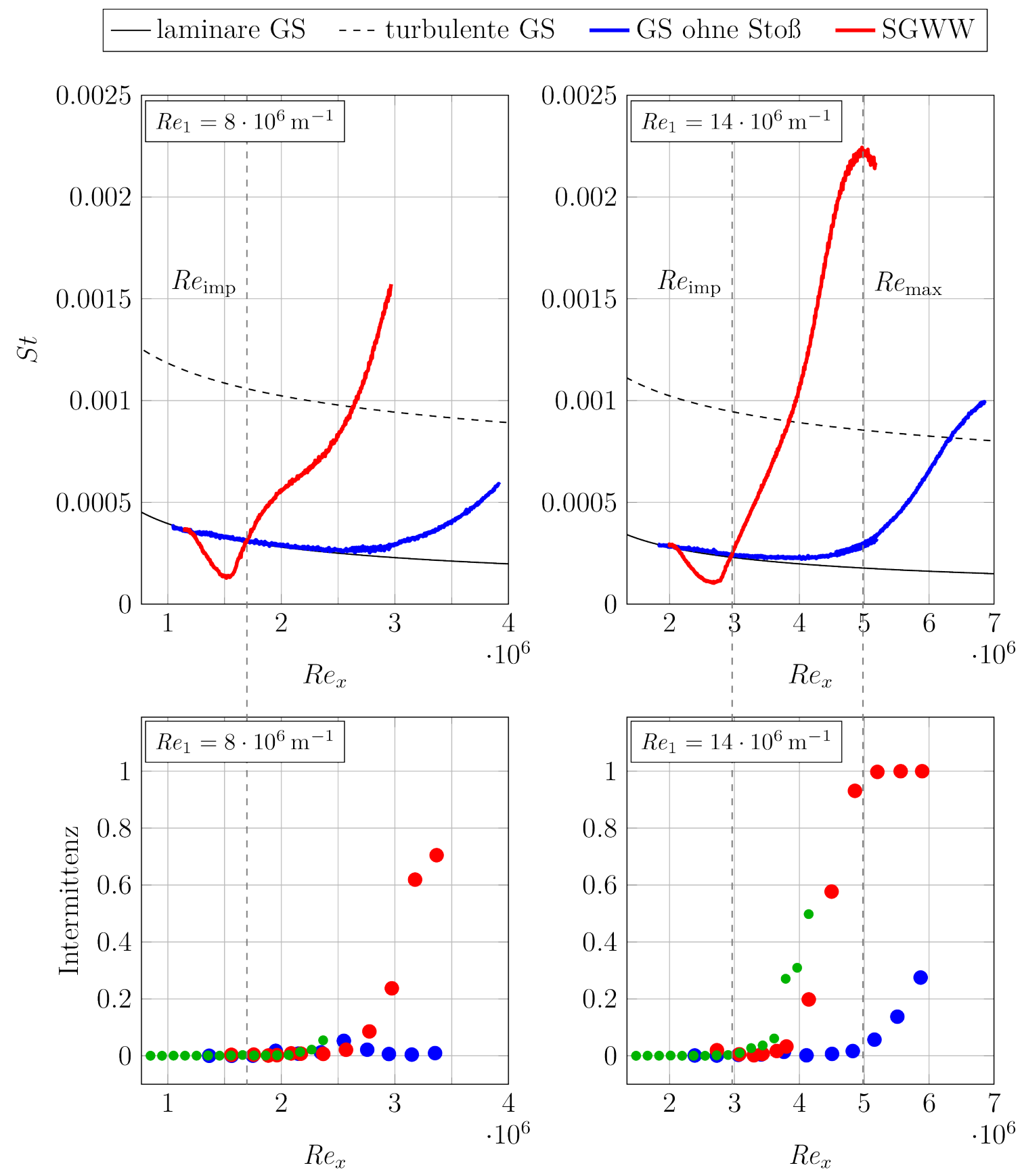

$\gamma_{\text {FLDI, GS }} \gamma_{\text {FLDI, SGWW }} \bullet \gamma_{\mathrm{PCB}, \mathrm{SGWW}}$

Abbildung 4.26: Die obere Zeile zeigt den St-Verlauf der untersuchten SGWW mit einer Stoßintensität von $p_{3} / p_{1}=2.29$. In der unteren Zeile sind die Intermittenzverteilungen dargestellt, die aus den Zeischrieben der FLDITechnik bzw. den PCB-Sensoren berechnet wurden. Markante Stellen der SGWW wurden mittels vertikaler Linien durch beide Diagramme gekennzeichnet. 


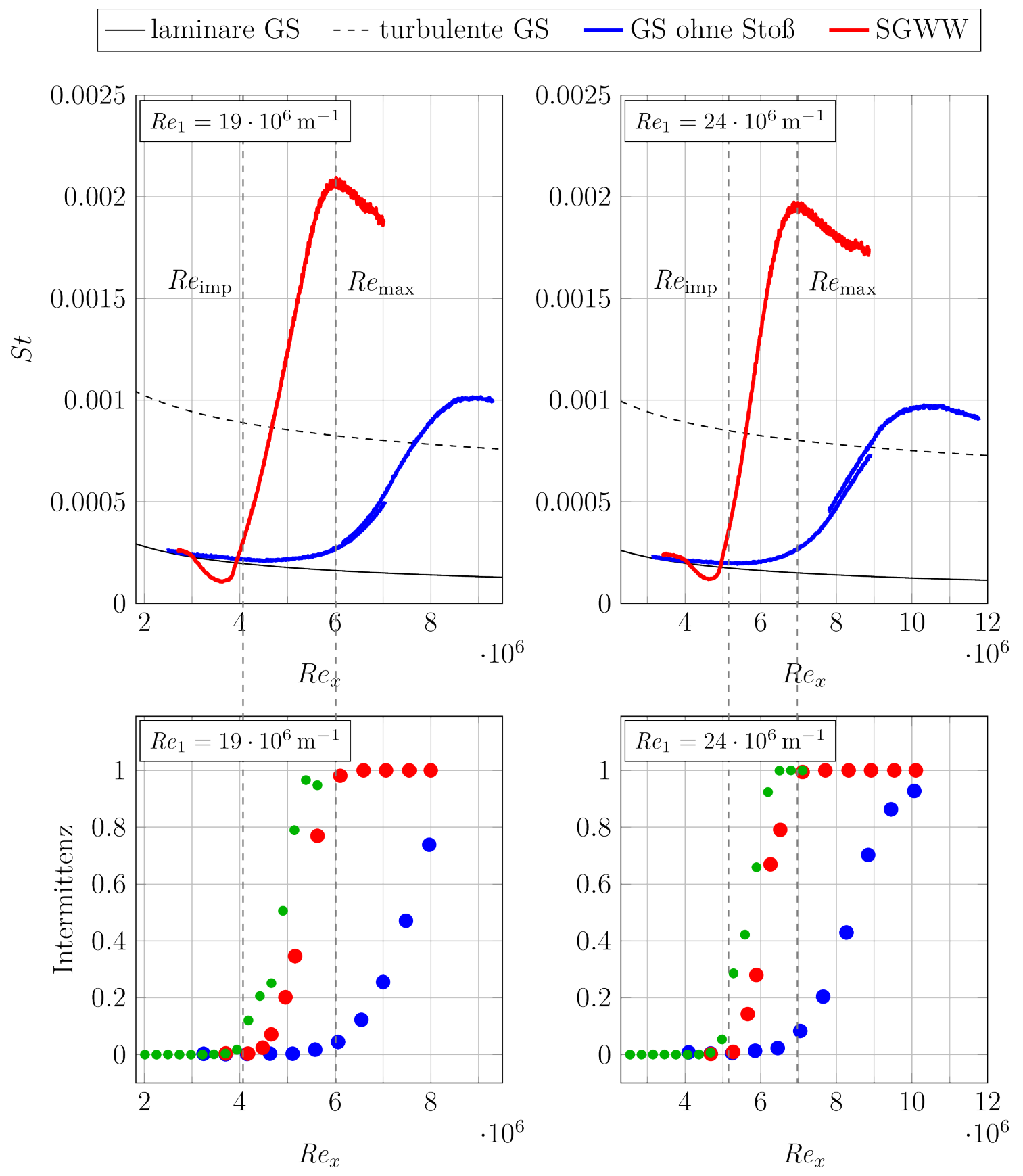

$\bullet \gamma_{\mathrm{FLDI}}$ GS $\bullet \gamma_{\mathrm{FLDI}}$ SGWW $\bullet \gamma_{\mathrm{PCB}} \mathrm{SGWW}$

Abbildung 4.27: Fortsetzung von Abb. 4.26 
sowohl zur Visualisierung der untersuchten SGWW als auch dem Vergleich zwischen Intermittenzwert und Stanton-Zahl. Die räumliche Entwicklung des Intermittenzwertes entlang der Hauptströmungsrichtung ist in der unteren Zeile der Abbildung dargestellt. Die Intermittenzverteilung zeigt, dass sich bei einer SGWW sowohl der Transitionsbeginn als auch das Transitionsende stromauf gegenüber der natürlichen Transition verschieben. Abb. 4.27 zeigt, dass auch der $R e_{x}$-Bereich, in dem der Übergang von $\gamma=0$ nach $\gamma=1$ stattfindet, sich gegenüber der natürlichen Transition verkürzt.

Die Intermittenzverteilung auf Basis der PCB-Daten ( $\gamma_{\mathrm{PCB}}$, SGww $)$ erscheint leicht gegenüber $\gamma_{\mathrm{FLDI}}$, SGWw stromauf verschoben. Die gleiche Verschiebung konnte bereits bei der Intermittenzverteilung zur ungestörten Transition beobachtet werden (vgl. Abb. 4.17). Als Grund für die Verschiebung wurde bereits die störanfällige Montage der PCBSensoren diskutiert. Das selbe Argument greift auch bei den Untersuchungen der Intermittenz bei der SGWW.

Ein Vergleich der Intermittenzwerte mit der dazugehörigen St-Verteilung zeigt, dass die $R e_{x}$-Koordinate, an der $\gamma_{\text {FLDI, SGWW }}=1$ erreicht wird, mit der $R e_{x}$-Position des maximalen Wärmestromdichte übereinstimmt. Zur Veranschaulichung dieses Zusammenhangs wurde in den Abbildungen 4.26 und 4.27 eine schwarze, gestrichelte, vertikale Linie eingefügt, die durch $S t_{\max }$ verläuft. Die maximale Wärmestromdichte bei transitionellen SGWW scheint an das Transitionsende gekoppelt zu sein.

Die Intermittenzverteilungen in Abb. 4.26 bestätigt, dass stromab von $R e_{\text {imp }}$ nicht unmittelbar der Transitionsprozess beginnen muss. Ist die laminare Grenzschicht bei der SGWW ausreichend stabil, so verbleibt die Grenzschicht zunächst laminar. Das Ausbleiben des Transitionsbeginns äußert sich in der St-Verteilung anhand eines zunächst gemäßigten Anstiegs in der Wärmestromdichte. Die SGWW führt allerdings zu einer verstärkten Anregung von Störungen innerhalb der Grenzschicht, sodass der Transitionsprozess stromauf vom ungestörten Fall vollzogen wird. Setzt nach einer gewissen Lauflänge der Transitionsprozess ein, so spiegelt sich dies als Knick im St-Verlauf wieder, ab dem die Wärmestromdichte verstärkt zunimmt. Dieser Knick ist beispielsweise in Abb. 4.26 für $R e_{1}=8 \cdot 10^{6} \mathrm{~m}^{-1}$ bei etwa $R e_{x}=2.5 \cdot 10^{6}$ erkennbar.

\subsubsection{LDS bei SGWW}

Der einfallende Verdichtungsstoß stört die natürliche Grenzschicht, wodurch sich auch die räumliche Entwicklung des LDS verändert. In Abbildung 4.28 sind die LDS auf Basis der FLDI- bzw. PCB-Daten an diversen Plattenpositionen bei der Stoßauftreffposition $x_{\text {imp }}=214.1 \mathrm{~mm}$ dargestellt.

Da sich das sensitive Volumen der FLDI-Messtechnik über mehrere Zentimeter entlang der Oberflächennormalen erstreckt (vgl. Abb. 3.19), tragen sowohl die Dichteschwankungen der Grenzschicht, der Außenströmung und der Stoßstruktur zum Messsignal bei. So führt der geometrische Verlauf des Rekompressionsstoßes zu einer Staffelung der LDS zwischen den verschiedenen Messpositionen. Der Abstand zwischen dem Rekompressionsstoß und der Modellwand nimmt mit der Lauflänge zu. Infolgedessen wächst im sensitiven Volumen der Anteil der durch den Rekompressionsstoß verdichteten Luft. Da durch den Stoß die relativen Dichteschwankungen nicht signifikant abnehmen, steigen 




(a) LDS auf Basis der FLDI-Daten

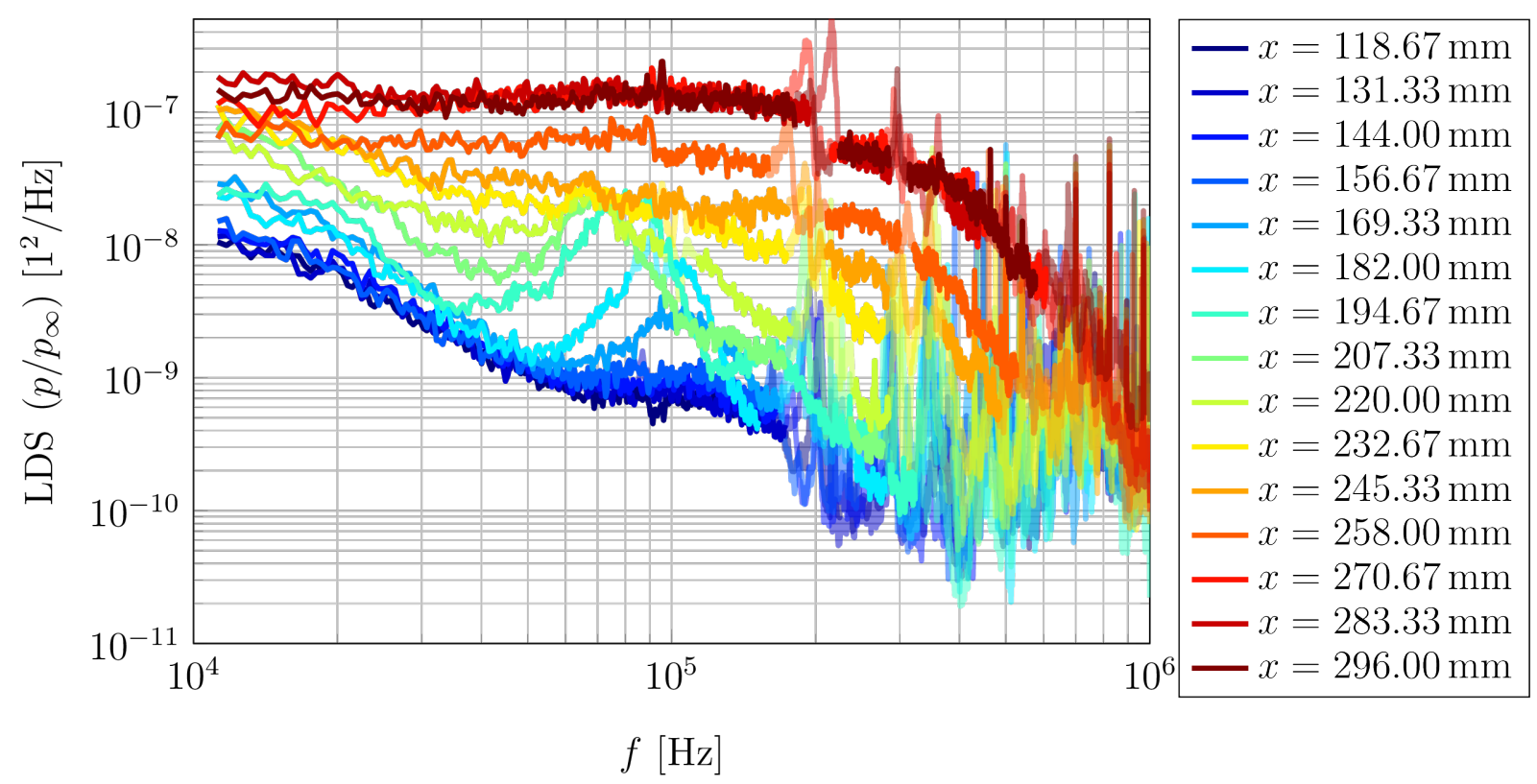

(b) LDS auf Basis der PCB-Daten

Abbildung 4.28: LDS bei einer SGWW an diversen Plattenpositionen für $R e_{1}=$ $19 \cdot 10^{6} \mathrm{~m}^{-1}, p_{3} / p_{1}=2.29$ und $x_{\mathrm{imp}}=214.1 \mathrm{~mm}$

die absoluten Dichteschwankungen stromab des Rekompressionsstoßes. Dieser geometrische Effekt erklärt die Staffelung der LDS im Frequenzbereich $f<50 \mathrm{kHz}$, wo nach 
Kapitel 4.1.4 die Störungen der Außenströmung dominieren. Durchlaufen die Expansionswellen der Stoßgeneratorhinterkante das sensitive Volumen, nehmen die absoluten Dichteschwankungen wieder ab. Aufgrund der reduzierten Dichteschwankungen sinkt das LDS für die Messpositionen $x \geq 371 \mathrm{~mm}$ wieder.

Stromab von $x_{\text {imp }}$ beschleunigt die stoßinduzierte Turbulenz den Transitionsprozess. Der zeitliche Anteil an Turbulenzflecken innerhalb der Grenzschicht steigt. Da die Turbulenzflecken ein breitbandiges Frequenzspektrum besitzen, nimmt auch die Bandbreite der LDS zu. Sowohl das Minimum bei $f \approx 5 \mathrm{MHz}$ als auch das lokale Maximum bei $f \approx 6.5 \mathrm{MHz}$ entstehen aufgrund des endlichen Abstandes zwischen den beiden Fokuspunkten und können anhand einer analytischer Rechnung vorhergesagt werden (vgl. Abb. 3.17b).

Durch die SGWW kann ein zusätzliches lokales Maximum in den FLDI-LDS beobachtet werden, das sich bei $f \approx 100 \mathrm{kHz}-170 \mathrm{kHz}$ befindet. Durch die stoßinduzierte Ablösung entstehen zusätzliche Instabilitäten in der Grenzschicht. So sind die Scherkräfte in der Ablöseblase der Ursprung von Kevin-Helmholtz-Instabilitäten (Waindim et al. 2020, Helm et al. 2021). Die zu erwartende Frequenz der Kevin-Helmholtz-Instabilität liegt für die untersuchten Strömungsbedingungen bei ca. $10 \mathrm{kHz}$ und scheidet dadurch als Erklärung für das lokale Maximum aus. Eine Wavelet-Analyse der Messsignale zeigte, dass die Frequenzen des Maximums kontinuierlich angeregt sind und unabhängig von den Turbulenzflecken vorliegen. Da das Maximum nicht die stochastischen Eigenschaften aufweist, die für den Transitionsprozess typisch sind, wird ein Zusammenhang mit der Transition ausgeschlossen. Ein Vergleich mit den LDS der PCB-Daten zeigt, dass die PCB-Sensoren kein lokales Maximum bei $f \approx 100 \mathrm{kHz}-170 \mathrm{kHz}$ detektieren. Daher wird vermutet, dass die Ursache des lokalen Maximums in der Außenströmung liegt. Entlang der Wandnormalen durchläuft der Laserstrahl der FLDI-Technik auch den Nachlauf des Stoßgenerator: 1 . Außerhalb der Fokusebene werden Dichteschwankungen zwar nur gedämpft von der FLDI-Technik detektiert (vgl. Abb. 3.19), allerdings ist die Größenordnung der instationären Dichtegradienten im Nachlauf unbekannt. Eine abschließende Erklärung für die Ursache des lokalen Maximums konnte nicht gefunden werden.

Die LDS der PCB-Messdaten sind in Abb. 4.28b dargestellt. Für die Messpunkte stromauf des Interaktionsgebietes $(x \leq 156.67 \mathrm{~mm})$ sind keine Unterschiede zu den LDS ohne SGWW feststellbar. An den Messpositionen $x=169.33 \mathrm{~mm}-220 \mathrm{~mm}$ wird ein lokales Maximum in den LDS detektiert, dessen Frequenz $(f \approx 66 \mathrm{kHz}-105 \mathrm{kHz})$ sich mit der Messposition verändert. Strömungsmechanisch befinden sich diese Messpositionen im Gebiet der Ablöseblase und Leistungsdichtespektren bei anderen Anströmbedingungen bzw. Stoßintensitäten deuten an, dass auch die Größe der Ablöseblase einen Einfluss auf die Frequenz des lokalen Maximums hat. Bei der Größe der Ablöseblase scheint es egal ob diese durch eine Variation von $R e_{1}$ oder der Stoßintensität verändert wird. Der Grund für die Entstehung des lokalen Maximums ist unklar. Es wirkt, als würde die beobachtete Verschiebung der Frequenz mit der lokalen Höhe der Trennlinie korrelieren, sodass diese Höhe eventuell mit der Entstehung des lokalen Maximums zusammenhängt. Allerdings scheiden die Kevin-Helmholtz-Instabillitäten als Ursache aus, da deren Frequenz

\footnotetext{
${ }^{1}$ Die Hinterkante des Stoßgenerators befindet sich $49 \mathrm{~mm}$ oberhalb der Plattenoberfläche.
} 
zu niedrig ist. In den LDS der FLDI-Daten wurde kein lokales Maximum innerhalb der Ablöseblase beobachtet, da die Dichteschwankungen der Außenströmung in diesem Frequenzbereich dominieren. Da keine Veröffentlichungen bezüglich LDS bei SGWW mittels FLDI oder PCB bekannt sind, gibt es keine Vergleichsmöglichkeiten oder vorhandene Modellvorstellungen. Als Konsequenz verbleiben im Rahmen dieser Arbeit einige Beobachtungen in den LDS ohne Erklärung.

Stromab des Wiederanlegepunktes $\left(x>x_{\text {imp }}\right)$ setzt direkt der Transitionsprozess ein und im Gegensatz zur ungestörten Strömung sind keine zweiten Mack-Moden in den LDS erkennbar. Stattdessen nimmt mit jeder Messposition die Leistungsdichte über die gesamte Bandbreite zu. Diese Entwicklung der LDS deutet darauf hin, dass durch die stoßinduzierten Störungen Schritte in dem natürlichen Ablauf des Transitionsprozesses übersprungen werden (vgl. Kapitel 2.1.3) und direkt Turbulenzflecken entstehen. Die direkte Ausbildung von Turbulenzflecken ist allerdings bei SGWW nicht immer der Fall. Bei Messungen mit schwachen Stößen und Auftreffpositionen, die weit stromauf des natürlichen Transitionsbeginns liegen, konnte auch stromab des Wiederanlegepunktes die Ausbildung von zweiten Mack-Moden in den LDS beobachtet werden. Ausschlaggebend für den Transitionsprozess bei SGWW scheint zu sein, wie weit die ungestörte Grenzschicht an der $x_{\text {imp }}$-Position noch vom natürlichen Transitionsbeginn entfernt ist.

\subsubsection{Skalierungsgesetze für kritische Kenngrößen}

Die Erarbeitung von aussagekräftigen Skalierungsgesetzen ermöglicht einen tieferen Einblick in die strömungsmechanischen Vorgänge bei SGWW und verbessert die Prognostizierbarkeit der Kenngrößen. Anhand der Abb. 4.22 und 4.23 wurde der Einfluss der unabhängig variierten Parameter $x_{\mathrm{imp}}, R e_{1}$ und $p_{3} / p_{1}$ auf den $S t$-Verlauf bei SGWW diskutiert. Abbildung 4.29 zeigt eine schematische St-Verteilung mit und ohne SGWW. Zusätzlich sind die Parameter vermerkt, die für die folgenden Skalierungen verwendet werden.

Für die Beschreibung der St-Verläufe mit SGWW bieten sich drei charakteristische Kenngrößen an. $\mathrm{Zu}$ diesen Größen gehört als erstes $S t_{\max }$, als Angabe zur maximalen thermischen Belastung des Modells. Als zweites beschreibt $L_{\mathrm{ui}}=\Delta R e_{\mathrm{ui}} / R e_{1}$ den Bereich stromauf von $x_{\mathrm{imp}}$, der durch die SGWW beeinflusst wird. Als dritte Größe gibt der Abstand $L_{\max }=\Delta R e_{\max } / R e_{1}=\left(R e_{\max }-R e_{\text {imp }}\right) / R e_{1}$ an, in welchem Bereich der Transitionsprozess stromab von $R e_{\text {imp }}$ vollzogen wird. Wie in Kapitel 4.2.2 dargelegt, hängen die drei Größen $S t_{\max }, L_{\mathrm{ui}}$ und $L_{\max }$ von der Kombination der Parameter $x_{\mathrm{imp}}$, $R e_{1}$ und $p_{3} / p_{1}$ ab.

\section{Skalierung der Ablöselänge}

Die Länge der Ablöseblase $L_{\text {sep }}$ ist eine charakteristische Größe der SGWW, die häufig untersucht wurde. Allerdings ist eine direkte Bestimmung von $L_{\text {sep }}$ aus experimentellen Daten oft nicht möglich, weshalb die Ablöselänge beispielsweise aus der Analyse von Schattenaufnahmen abgeschätzt wird. Obwohl $L_{\text {ui }}$ anders definiert ist als $L_{\text {sep }}$, skalieren die beiden Größen praktisch analog, sodass alternativ auch die Länge der Stromauf- 




Abbildung 4.29: Schematische Darstellung der Stantonzahlverteilung mit und ohne SGWW für eine konstante $R e_{1}$ (angepasst aus (Schülein 2014)). Gekennzeichnet sind die charakteristischen Größen des St-Verlaufs.

wirkung zur Beschreibung von $L_{\text {sep }}$ verwendet wird (Mallinson et al. 1996, Souverein et al. 2013). Trotz der unterschiedlichen Ansätze zur Beschreibung von $L_{\text {sep }}$ sind die daraus resultierenden Abweichungen klein gegenüber den strömungsmechanischen Einflussfaktoren auf $L_{\text {sep }}$ (Souverein et al. 2013). Zu den wesentlichen Faktoren gehören die Mach-Zahl, die Reynolds-Zahl, die Stoßintensität und die Eigenschaften der ankommenden Grenzschicht (Délery \& Marvin 1986, Souverein et al. 2013). Da es sich bei stoßinduzierten Ablösungen nach Chapman et al. (1957) um freie Interaktionen handelt, ergeben sich die Strömungsparameter am Ablösepunkt aus der gegenseitigen Beziehung zwischen der lokalen Grenzschicht und der Außenströmung.

Obwohl sowohl die wesentlichen Einflussfaktoren als auch theoretische Konzepte zur Ablösung bekannt sind, konnte bisher keine universelle Skalierung für die Länge der Ablöseblase gefunden werden. Bislang veröffentlichte Korrelationsansätze differieren bezüglich des Ansatzes, wie die einzelnen Einflussfaktoren in die Skalierung einfließen. Im Folgenden soll ein Einblick in einige bekannte Skalierungsansätze gegeben werden, die Repräsentation der Messdaten dieser Arbeit in den existierenden Ansätzen geprüft und nachfolgend ein alternativer Ansatz vorgeschlagen werden.

Needham (1965) zeigte anhand von experimentellen Daten zu laminaren SGWW an Rampen, dass

$$
\frac{L_{\text {sep }}}{x_{\text {ui }}} \propto \frac{\sqrt{R e_{\mathrm{ui}}}}{M a_{\infty}^{3}}\left(\frac{p_{3}}{p_{\mathrm{P}}}\right)^{2} .
$$

Der Quotient $p_{3} / p_{\mathrm{P}}$ skaliert den Druck stromab des Wiederanlegestoßes mit dem Plateaudruck stromab des Ablösestoßes und stellt ein Maß für die Stoßintensität dar.

Katzer (1989) analysierte für $1.4 \leq M a \leq 3.4$ die Ablöselänge bei der Interaktion 
zwischen einem einfallenden Stoß und einer laminaren Grenzschicht mittels numerischer Simulation und präsentierte folgenden linearen Zusammenhang:

$$
\frac{L_{\mathrm{sep}}}{\delta_{1, \mathrm{imp}}}=4.4 \frac{\sqrt{R e_{\mathrm{imp}} \frac{\mu_{\infty} T_{\mathrm{w}}}{\mu_{\mathrm{w}} T_{\infty}}}}{M a_{\infty}^{3}} \frac{p_{3}-p_{\mathrm{inc}}}{p_{1}} .
$$

Hierbei gibt $p_{\text {inc }}$ den notwendigen Druck an, ab dem eine Ablösung überhaupt stattfindet. Neben der unterschiedlichen Handhabung der Stoßintensität entdimensionalisiert Katzer (1989) die Ablöselänge mit der Verdrängungsdicke $\delta_{1 \text {,imp }}$ an der Stoßauftreffposition einer ungestörten Grenzschicht.

Ein vielversprechender Skalierungsansatz wurde von Souverein et al. (2013) auf Basis von Erhaltungsgrößen entwickelt. Die Existenz der Ablösblase reduziert den Massenbzw. Impulsfluss der Strömung über das Interaktionsgebiet. Durch einen Vergleich zwischen einer viskosen SGWW mit Ablösung und einer nichtviskosen SGWW, wurde die Reduzierung im Massen- und Impulsfluss anhand der Länge der Ablöseblase quantifiziert. Aus der Quantifizierung konnte folgende Skalierung für die Ablöselänge gefolgert werden:

$$
L^{*}=\frac{L_{\mathrm{ui}}}{\delta_{1, \mathrm{ui}}} \frac{\sin (\beta) \sin (\varphi)}{\sin (\beta-\varphi)} .
$$

Hierbei beschreibt $\beta$ den Stoßwinkel und $\varphi$ die Ablenkung der Strömung. Souverein et al. (2013) verifizierten diesen Ansatz erfolgreich mittels einer umfangreichen experimentellen Datenbasis an turbulenten SGWW von einfallenden Stößen sowie Rampen und zeigten, dass $L^{*}$ als Funktion von

$$
S^{*}=\frac{2 k}{\kappa M a_{\infty}^{2}} \frac{p_{3}-p_{1}}{p_{1}} \quad \text { mit } \quad k= \begin{cases}3 & \text { für } R e_{\delta_{2, \mathrm{ui}}} \leq 1 \cdot 10^{4} \\ 2.5 & \text { für } R e_{\delta_{2, \mathrm{ui}}}>1 \cdot 10^{4}\end{cases}
$$

dargestellt werden kann. Hier beschreibt $R e_{\delta_{2, \text { ui }}}$ die Reynolds-Zahl basierend auf der Impulsverlustdicke am Interaktionsbeginn.

Obwohl sich die drei vorgestellten Skalierungsansätze in ihrer Umsetzung unterscheiden, lassen sich gemeinsame Konzepte erkennen. So werden in Anlehnung an die Theorie der freien Interaktion oft die Strömungsparameter am Interaktionsbeginn verwendet, während die Stoßintensität über ein Verhältnis des aufgeprägten Druckanstieges in die Korrelationen einfließt. Es ist allerdings noch ungeklärt, warum die einzelnen Datensätze und Untersuchungen zu den unterschiedlichen Skalierungsgesetzen gelangen. Ein weiterer Einflussfaktor geht auch vom Zustand der Grenzschicht aus, da sich im Zuge der Transition der wandnahe Impuls erhöht und die Grenzschicht erst bei größeren Druckanstiegen ablöst. Die bekannten Skalierungsgesetze sind in der Regel nur explizit für laminare oder turbulente SGWW optimiert und anwendbar. Die Entwicklung eines Skalierungsansatzes für die Ablöselänge bei transitionellen SGWW erweist sich als besonders komplex, da neben der eigentlichen Skalierung der Ablöselänge zusätzliche Anforderungen erfüllt werden sollen. Vereinfacht gesehen, stellen die transitionellen SGWW einen Übergang von den laminaren SGWW zu den turbulenten SGWW dar. 
Nach dieser Vorstellung wird für die Ablöselänge ein stetiger Übergang zwischen den verschiedenen Grenzschichtzuständen erwartet. Somit sollten die skalierten Ablöselängen am Transitionsbeginn bzw. Transitionsende glatt an den Skalierungsgesetzen für rein laminare bzw. turbulente SGWW anschließen. Neben einem gleichmäßigen Übergang soll auch die Position des Transitionsbeginns und des Transitionsendes korrekt im Skalierungansatz berücksichtigt werden.

Needham (1965) zeigte anhand seiner Korrelation, wie sich die Ablöselänge mit einsetzender Transition reduziert, weshalb sich ein direkter Vergleich mit den experimentellen Daten dieser Promotionsarbeit zu transitionellen SGWW anbietet. Wie oben diskutiert, wird für den Vergleich $L_{\text {ui }}$ als Maß für $L_{\text {sep }}$ verwendet. Weil in der vorliegenden Studie der Plateaudruck nicht gemessen wurde, wird statt $\left(p_{3} / p_{\mathrm{P}}\right)^{2}$ das Verhältnis $p_{3} / p_{1}$ für die Skalierung der Stoßintensität verwendet. Für die untersuchten Stoßintensitäten ist die notgedrungene Abwandlung des Skalierungsgesetzes nicht gravierend. Die skalierten $L_{\mathrm{ui}}$ sind in Abbildung 4.30 in die alte Darstellung von Needham (1965) eingetragen, um einen direkten Vergleich zu ermöglichen. Die Darstellung von Needham (1965) zeigt, dass für laminare Grenzschichten die skalierten $L_{\mathrm{sep}}$ mit $R e_{x_{0}}$ (entspricht $R e_{\mathrm{ui}}$ ) wachsen. Die transitionellen SGWW stellen vereinfacht gesehen einen Übergang zu dem Niveau für turbulente SGWW dar.

Die eigenen Datenpunkte weichen deutlich von Needhams Kurven ab. Die skalierten Ablöselängen sind sichtbar größer und es ist kein weiterer Anstieg für die untersuchten laminaren SGWW beobachtbar. Zwar führt die Transition auch bei den aktuellen Datenpunkten zu einem Abfall der skalierten Ablöselänge, allerdings existiert eine horizontale Verschiebung zwischen den Datenpunkten bezüglich $R e_{1}$. Insgesamt erweist sich die Skalierung der eigenen experimentellen Daten mit dem Ansatz von Needham (1965) als nicht zufriedenstellend. Eine Überprüfung der anderen Skalierungsansätze führte zu noch unstimmigeren Darstellungen. Um die Veränderung der Ablöselänge bei transitionellen SGWW aufzuzeigen, erscheint die von Needham (1965) gewählte Auftragung gegenüber $R e_{\mathrm{ui}}$ als suboptimal, da in den ungestörten Messungen der Grenzschicht bereits eine Abhängigkeit der Transitionslage von $R e_{1}$ festgestellt wurde (vgl. Abb. 4.3).

Um den Einfluss der Transition auf die Ablöselänge angemessener darzustellen, wird vorgeschlagen den Grenzschichtzustand bei der SGWW für die jeweilige $R e_{1}$ über

$$
\zeta_{\mathrm{imp}}=\zeta\left(R e_{\mathrm{imp}}\right)=\frac{R e_{\mathrm{imp}}-R e_{\mathrm{Tb}}}{R e_{\mathrm{Te}}-R e_{\mathrm{Tb}}}
$$

zu quantifizieren (vgl. Kapitel 4.1.5). Abbildung 4.31a bestätigt, dass durch die Verwendung von $\zeta_{\text {imp }}$ keine $R e_{1}$-Abhängigkeit bei den skalierten Ablöselängen sichtbar ist. Auf eine Berücksichtigung der Machzahl wird bei den eigenen Skalierungsvorschlägen verzichtet, weil die Experimente der aktuellen Studie bei einer festen Machzahl durchgeführt wurden und somit keine Aussage bezüglich eines Machzahl-Effektes getroffen werden kann.

Während Needham (1965) noch $x_{\text {ui }}$ für die Skalierung der Ablöselänge verwendete, wird bei den aktuelleren Arbeiten von Katzer (1989) bzw. Souverein et al. (2013) die Verdrängungsdicke eingesetzt. Die Auswirkung dieses Wechsels wird durch den Vergleich 


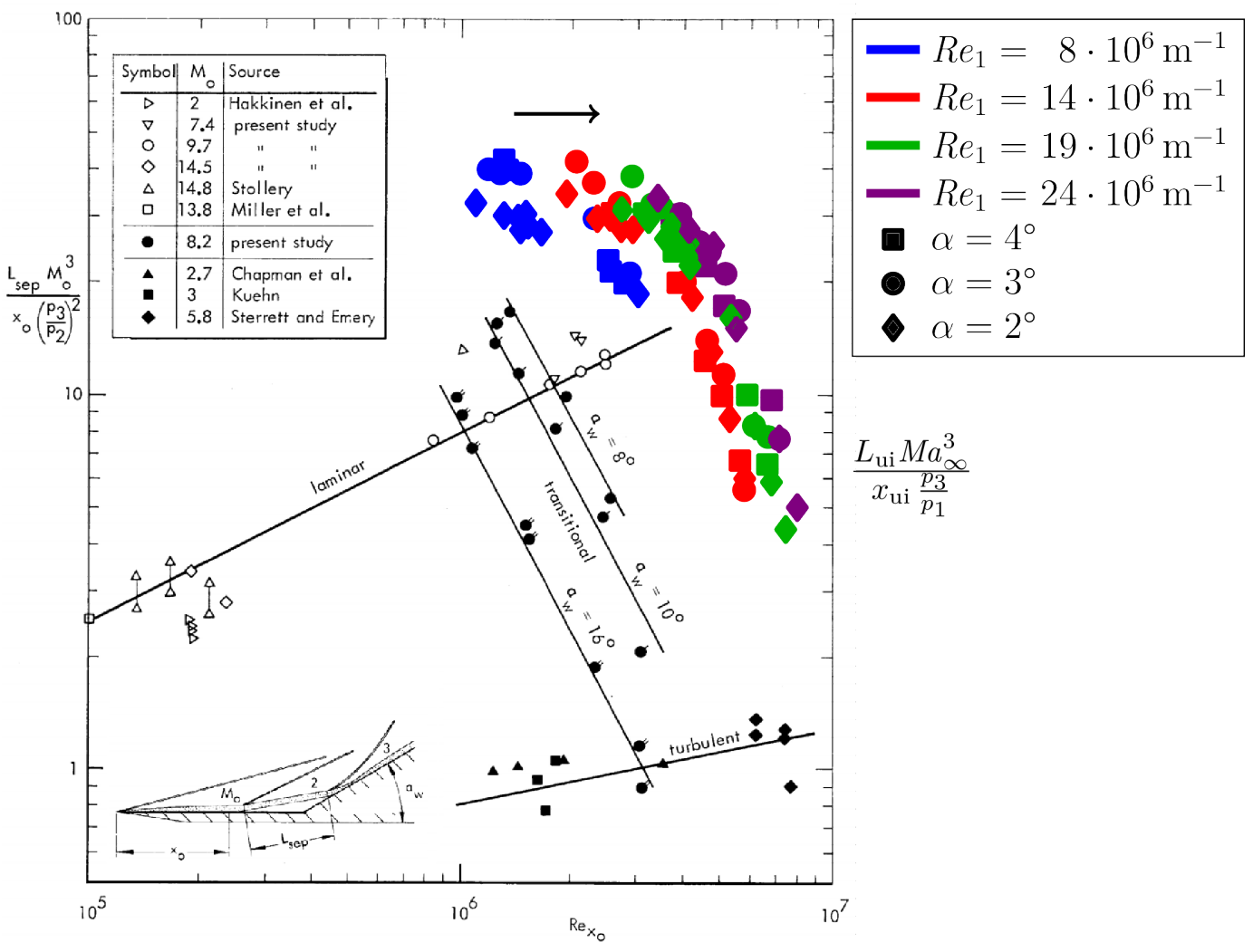

Abbildung 4.30: Zur Überprüfung des Skalierungsansatzes von Needham (1965) wurden die experimentellen Daten in die Darstellung von Needham (1965) eingetragen. Da in den eigenen Messungen der Plateaudruck stromab des Ablösestoßes nicht gemessen wurde, wird die Stoßintensität stattdessen über das Druckverhältnis $p_{3} / p_{1}$ berücksichtigt.

zwischen Abbildungen 4.31a und b deutlich. Hierbei wurde zur Skalierung die laminare Verdrängungsdicke nach Gleichung 2.13 verwendet. Bei $\zeta_{\text {imp }}<0$ ist die Streuung der Datenpunkte beträchtlich, allerdings kann für die laminaren SGWW ein Anstieg der skalierten Ablöselängen bis $\zeta_{\text {imp }} \approx 0$ beobachtet werden. Für $\zeta_{\text {imp }} \geq 0$ setzt eine Reduzierung der skalierten Ablöselängen ein, die zu der Vorstellung passt, dass sich durch den Transitionsprozess der wandnahe Impuls erhöht und die Länge der Ablöseblase folglich abnimmt. Der Skalierungsansatz in Abbildung 4.31 b spiegelt am besten die theoretischen Erwartungen bezüglich der Ablöselänge bei transitionellen SGWW wieder, allerdings handelt es sich hierbei um eine Kombination aus mehreren einzelnen Korrelationsgesetzen. Die Allgemeingültigkeit des kombinierten Skalierungsansatzes muss durch weitere Studien überprüft werden.

\section{Skalierungsansatz für die stoßinduzierten Wärmelasten}

Um darzustellen welche Auswirkungen die Existenz einer SGWW auf die maximale induzierte Wärmelast hat, wurden in Schülein (2014) und Lunte \& Schülein (2019) die 


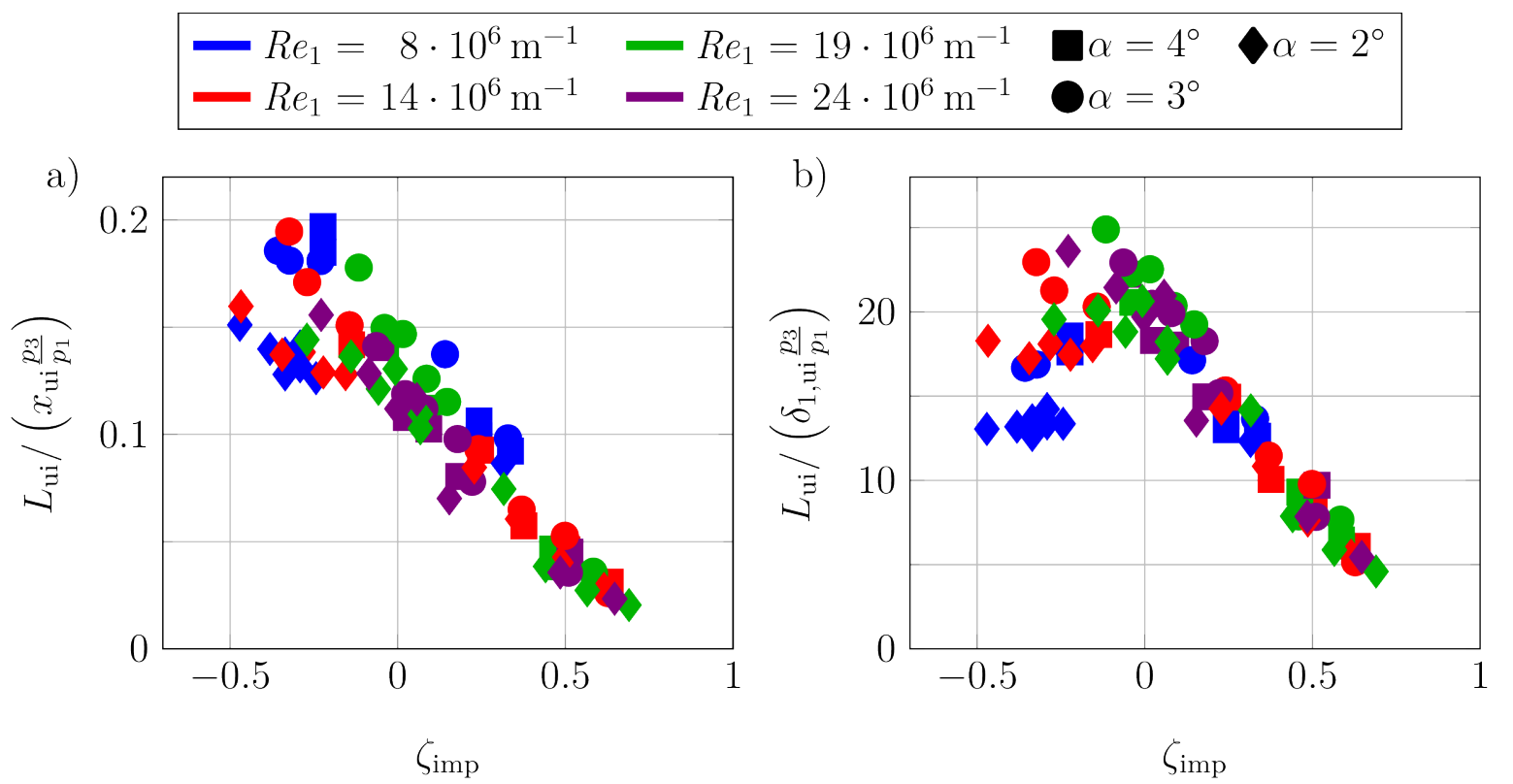

Abbildung 4.31: Skalierungsansätze für die Länge der Ablöseblase bei transitionellen SGWW. Zur Entdimensionalisierung der Ablöselänge wird einmal die Lauflänge $x_{\text {ui }}$ (a) und einmal die Verdrängungsdicke $\delta_{1, \text { ui }}$ (b) verwendet.

$S t_{\text {max }}$-Werte mit der Wärmestromdichte der ungestörten Strömung $\left(S t_{\mathrm{wo}}\right)$ an der selben $R e_{\max }$-Position normiert (siehe Abb. 4.29). Das Resultat einer solchen Skalierung wird anhand der aktuellen experimentellen Daten in Abb. 4.32 präsentiert. Bei einigen untersuchten Fällen ist der $S t_{\max }$-Wert durch die Expansionswellen von der Hinterkante des Stoßgenerators beeinflusst (vgl. Abb. 4.22c). Da die Auswirkung der Expansionswellen in vielen Fällen als gering eingestuft wird, wurden auch diese beeinflussten $S t_{\text {max }}$-Werte für die Analyse verwendet, aber als offene Symbole gekennzeichnet.

Die Daten in Abb. 4.32 zeigen, dass sich die größten Verstärkungsfaktoren $S t_{\max } / S t_{\mathrm{wo}}$ bei Stoßauftreffpositionen stromauf des Transitionsbeginns $\left(\zeta_{\mathrm{imp}}<0\right)$ ergeben, was auf die stromaufverlagerte Transition zurückgeführt werden kann. Während an der Position von $S t_{\max }$ das Transitionsende bereits erreicht ist (vgl. Abb. 4.26 und 4.27), ist die Transition beim Referenzfall noch nicht abgeschlossen und die Werte für $S t_{\text {wo }}$ gehören zu einer laminaren bzw. transitionellen Grenzschicht. Mit Zunahme von $\zeta_{\text {imp }}$ reduziert sich $S t_{\max } / S t_{\text {wo }}$, da der Abstand zwischen $R e_{\max }$ und dem natürlichen Transitionsende abnimmt. Folglich wächst $S t_{\text {wo }}$ mit $\zeta_{\text {imp }}$ und man beobachtet die geringsten Verstärkungsfaktoren im Bereich $\zeta_{\text {imp }}=1$.

Durch die Normierung mit dem ungestörten Referenzfall verdeutlicht Abb. 4.32, welche thermische Belastung durch den Verdichtungsstoß und der dadurch beschleunigten Transition hervorgerufen wird. Die teils zweistelligen Verstärkungsfaktoren machen bewusst, dass die transitionellen SGWW bei der Auslegung von Überschallfluggeräten nicht vernachlässigbar sind. Für eine fundierte Abschätzung der stoßinduzierten Wärmelasten 


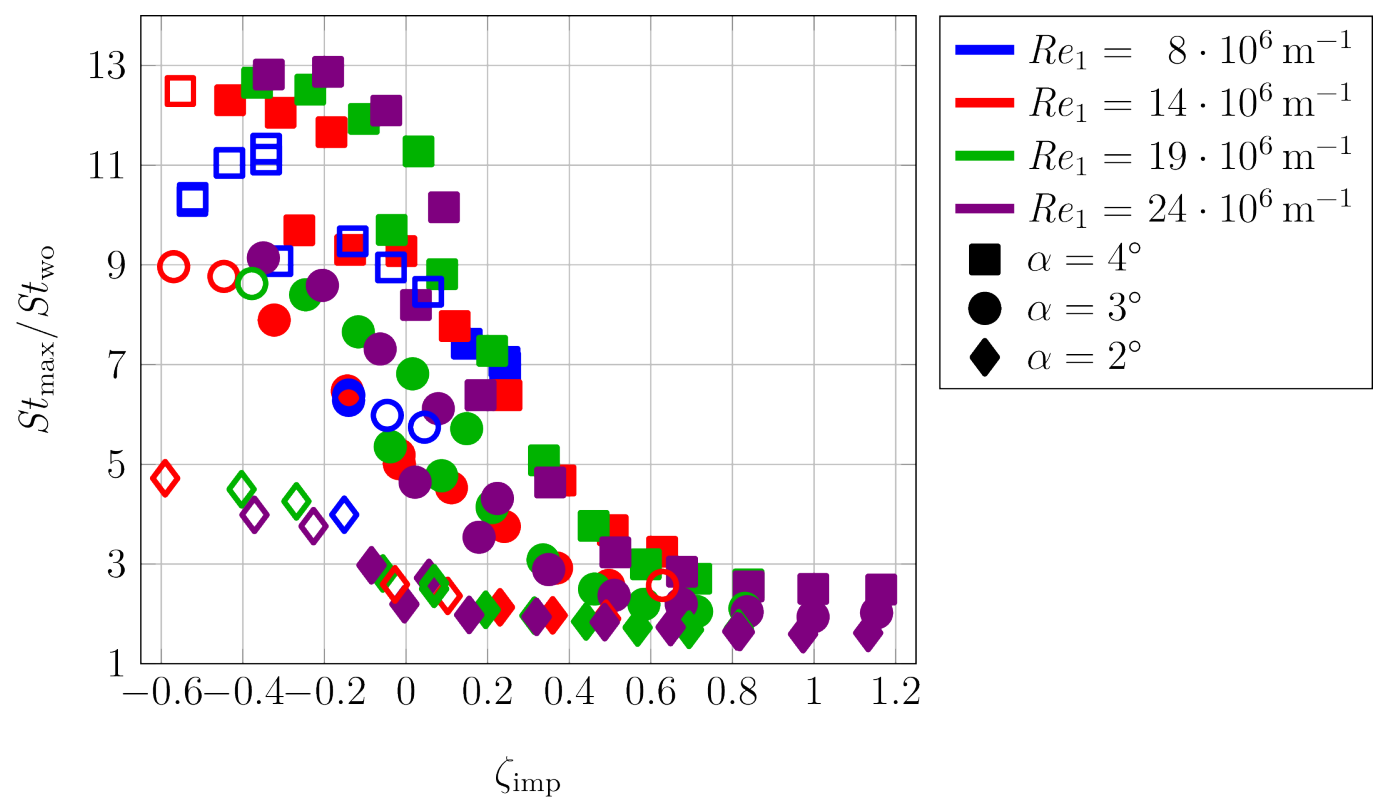

Abbildung 4.32: Stoßinduzierte maximale Wärmestromdichte im Verhältnis zum Niveau der ungestörten Referenzströmung in Abhängigkeit des Grenzsichtzustandes an der Stoßauftreffposition.

ist die Skalierung $S t_{\max } / S t_{\text {wo }}$ allerdings kaum geeignet, da der Verstärkungsfaktor zu stark von $\zeta_{\text {imp }}$ abhängt und die zugrundeliegenden Wirkprinzipien der transitionellen SGWW auf die induzierte Wärmestromdichte nicht berücksichtigt werden. Aus diesem Grund soll im folgenden ein neuer Skalierungsansatz für die stoßinduzierten maximalen Wärmelasten erarbeitet werden, in dem schrittweise die Mechanismen der transitionellen SGWW und deren Integration ins Skalierungsgesetz diskutiert werden.

Zunächst soll ein geeigneter Referenzwert für die $S t_{\text {max }}$-Werte ausgewählt werden. Wie Abb. 4.32 zeigt, hat die Stromaufverlagerung der Transition einen wesentlichen Einfluss auf die stoßinduzierte Wärmelast. Da die $S t_{\text {max }}$-Werte mit dem Transitionsende korrelieren, erscheint es sinnvoll $S t_{\max }$ mit der Stanton-Zahl am Transitionsende der dazugehörigen ungestörten Grenzschicht zu normieren. Dieser Ansatz berücksichtigt allerdings nicht den Einfluss der lokalen Grenzschichtdicke auf die Wärmestromdichte. Nach den theoretischen Lösungen für die laminare und vollturbulente Grenzschicht nimmt die Stanton-Zahl mit der Lauflänge $\left(R e_{x}\right)$ ab, da der Temperaturübergang zwischen der Wand und der Außenströmung über eine größere Grenzschichtdicke erfolgt und somit die Temperaturgradienten abflachen. Nach diesem Prinzip hängt die Höhe der $S t_{\max }$-Werte auch von der lokalen Grenzschichtdicke ab. Obwohl in dieser Studie die Grenzschichtdicke nicht experimentell gemessen wurde, soll der Einfluss der lokalen Grenzschichtdicke so gut wie aktuell möglich bei der Skalierung berücksichtigt werden, indem die $S t_{\mathrm{Te}}$-Kurven (Gl. 4.3) zur Normierung verwendet werden. Die $S t_{\mathrm{Te}}$-Kurven geben die dimensionslose Wärmestromdichte an einer $R e_{x}$-Position an, falls der Transitionsprozess der ungestörten Grenzschicht genau an der besagten Stelle zum Abschluss 
kommen würde. Somit gibt der Quotient

$$
\frac{S t_{\max }}{S t_{\mathrm{Te}}\left(R e_{\max }\right)}
$$

an, um welchen Faktor die Wärmestromdichte durch die SGWW zunimmt und vergleicht dabei $S t$-Werte miteinander, die jeweils am Transitionsende vorliegen. Da die Normierung an der selben $R e_{x}$-Position erfolgt, werden über den Referenzwert auch Effekte aufgrund der mit $R e_{x}$ wachsenden Grenzschichtdicke berücksichtigt. Das Ergebnis der Skalierung ist in Abb. 4.33 für die drei Stoßintensitäten aufgetragen.

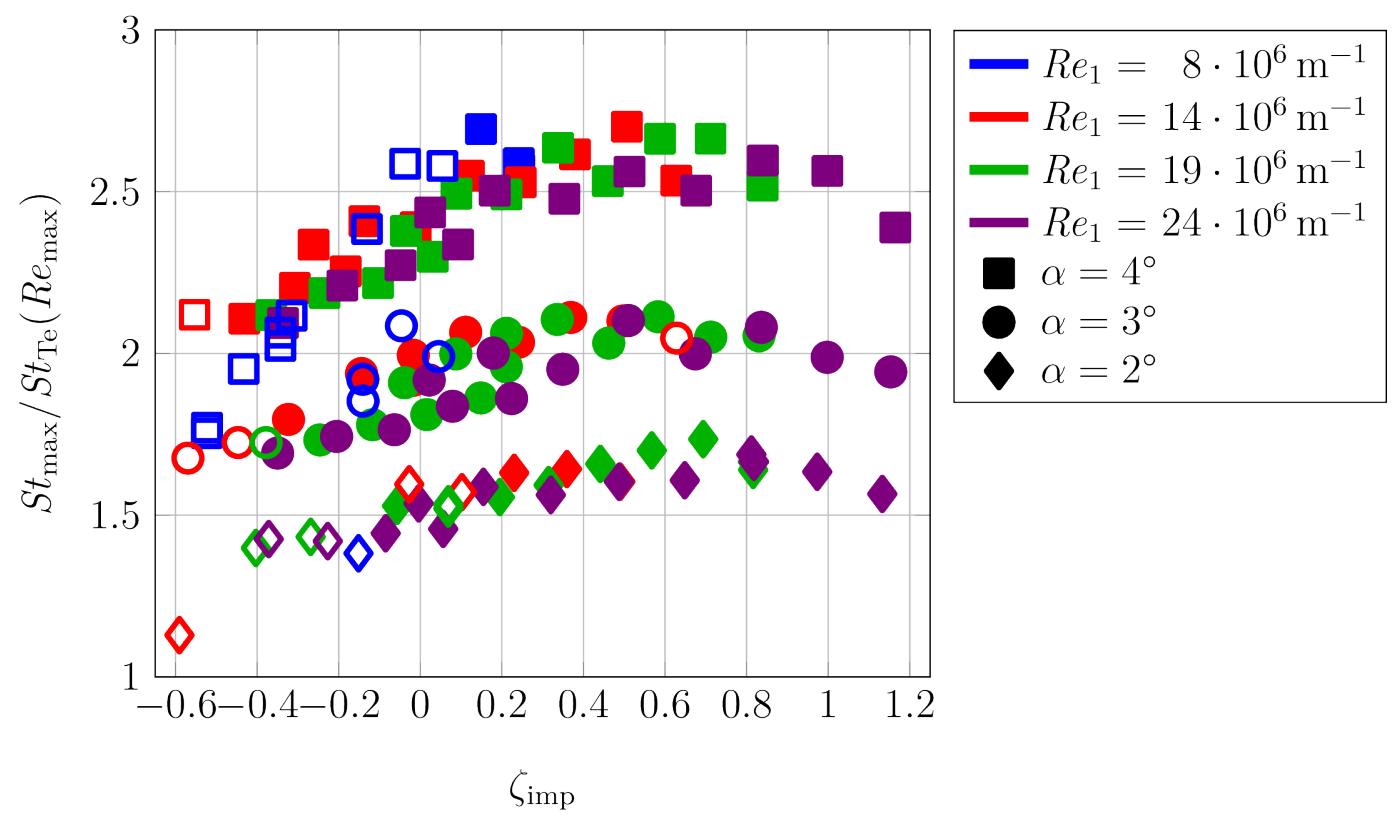

Abbildung 4.33: Skalierte maximale Wärmestromdichte bei transitionellen SGWW für unterschiedliche Stoßintensitäten und $R e_{1}$

In Abb. 4.33 ergibt sich mit dem vorgeschlagenen Skalierungsansatz jeweils ein gemeinsamer Verlauf der Datenpunkte für alle untersuchten $R e_{1}$. Außerdem ist eine Schichtung nach der Stoßintensität erkennbar. Für jede Stoßintensität steigt der Verstärkungsfaktor der Wärmestromdichte zunächst mit $\zeta_{\text {imp }}$ und erreicht schließlich ein maximales Level. Für $\zeta_{\text {imp }}>1$ wird eine Abnahme der Verstärkungsfaktoren erkennbar. Allerdings kann aufgrund der geringen Anzahl an Datenpunkten bei $\zeta_{\text {imp }}>1$ in Kombination mit der Messgenauigkeit keine verlässliche Aussage getroffen werden. Zudem hat die ungestörte Grenzschicht für $\zeta_{\text {imp }}>1$ das Transitionsende bereits stromauf des einfallenden StoBes erreicht, sodass der gewählte Referenzwert $\left(S t_{\mathrm{Te}}\left(R e_{\max }\right)\right)$ für diese Datenpunkte an Relevanz verliert und eventuell eine zusätzliche Tendenz durch die Verwendung eines ungeeigneten Referenzwertes entsteht.

Ein Erklärungsansatz für die Zunahme des Verstärkungsfaktors $S t_{\max } / S t_{\mathrm{Te}}\left(R e_{\max }\right)$ mit $\zeta_{\text {imp }}$ könnte wie folgt lauten. Abbildung 4.26 zeigt anhand der Intermittenzwerte, dass bei laminaren SGWW die stoßinduzierte Turbulenz vorerst nicht ausreicht, um den 
Trasitionsprozess direkt einzuleiten. Stattdessen bleibt die Grenzschicht zunächst laminar und Störungen werden zum Teil gedämpft. Wenn der laminar-turbulente Umschlag schließlich einsetzt, ist die stoßinduzierte maximale Wärmestromdichte aufgrund der gedämpften Störungen reduziert. Nähert sich der ungestörte Grenzschichtzustand an der Stoßauftreffstelle dem Transitionsbeginn, so reagiert die Grenzschicht sensibler auf die SGWW und der laminar-turbulente Umschlag setzt früher ein. Als Konsequenz werden die Störungen weniger gedämpft und die $S t_{\max } / S t_{\mathrm{Te}}\left(R e_{\max }\right)$-Werte nehmen zu. Diese Tendenz setzt sich zunächst bei transitionellen SGWW fort, da der Stoß den natürlichen Transitionsprozess beschleunigt. Der Verstärkungsfaktor der Wärmestromdichte nimmt $\mathrm{zu}$, bis durch die stoßinduzierte Turbulenz keine weitere Beschleunigung des Transitionsprozesses erreicht wird.

Bei dieser Modellvorstellung ist es plausibel zu erwarten, dass der kritische $\zeta_{\text {imp }}$-Wert, bei dem die höchsten $S t_{\max } / S t_{\mathrm{Te}}\left(R e_{\max }\right)$-Werte erreicht werden, von der Stoßintensität abhängt. Die Streuung der Datenpunkte in Abb. 4.33 verhindert allerdings eine verlässliche Bestimmung dieser kritischen $\zeta_{\text {imp }}$-Werte, sodass diese Vermutung nicht überprüft werden kann.

Nach dem dargelegten Erklärungsansatz hängt die Höhe der skalierten maximalen Wärmelast davon ab, inwieweit die SGWW den natürlichen Transitionsprozess beschleunigt. Folglich ist zu erwarten, dass der Verstärkungsfaktor $S t_{\max } / S t_{\mathrm{Te}}\left(R e_{\max }\right)$ mit dem Abstand $\left(\Delta R e_{\max }\right)$ zwischen Stoßauftreffposition und dem Transitionsende korreliert. In Abbildung 4.34a sind die Verstärkungsfaktoren anhand von $\Delta R e_{\max }$ eingefärbt und die kolorierten Datenpunkte belegen eine abnehmende Tendenz von $\Delta R e_{\max }$ mit wachsendem $\zeta_{\text {imp. }}$. Die Abhängigkeit zwischen $\Delta R e_{\max }$ und $\zeta_{\text {imp }}$ ist explizit in Abb. 4.34b dargestellt, worin die fallende Tendenz nochmals deutlich wird. Für $\alpha=3^{\circ}$ und $4^{\circ}$ ist zudem erkennbar, dass die $\Delta R e_{\max }$-Werte eine gewisse minimale Distanz nicht unterschreiten. Des Weiteren ergeben sich für schwache Stoßintensitäten tendenziell größere Distanzen bis zum Transitionsende, da weniger Störungen in die Grenzschicht eingebracht werden.

Die bisher gewonnenen Erkenntnisse bezüglich der beschleunigten Transition durch einen einfallenden Verdichtungsstoß lassen mögliche Analogien zur Beschleunigung der Transition mittels Rauigkeitselementen (Schneider 2008b) vermuten, die im Folgenden angesprochen werden. Wie stark Rauigkeitselemente die natürliche Transition beeinflussen, hängt unter anderem von deren Höhe im Bezug zur Grenzschichtdicke ab. Unterschreiten die Rauhigkeitselemente eine kritische Höhe, bleiben die Fluktuationen in der Außenströmung der entscheidende Faktor für die Lage der Transition. Rauhigkeitselemente werden als effektiv bezeichnet, sobald durch eine weitere Erhöhung keine zusätzliche Stromaufverschiebung der Transitionslage erreicht werden kann. In diesem Fall dominiert das Rauhigkeitselement die Lage der Transition. Zwischen der kritischen und effektiven Höhe existiert ein Bereich, indem die Transitionslage durch das Wechselspiel zwischen den verursachten Störungen und dem natürlichen Transitionsprozess definiert ist. Dieser Zwischenbereich weist Ähnlichkeiten auf zur untersuchten Transitionsbeeinflussung mittels einfallendem Verdichtungsstoß. Inwiefern die beschleunigte Transition mittels Rauhigkeitselemente bzw. einfallendem Stoß auf vergleichbaren Mechanismen beruht, kann im Rahmen der aktuellen Arbeit nicht geklärt werden.

In den Arbeiten von Schülein (2014) und Lunte \& Schülein (2019) wurden an ei- 


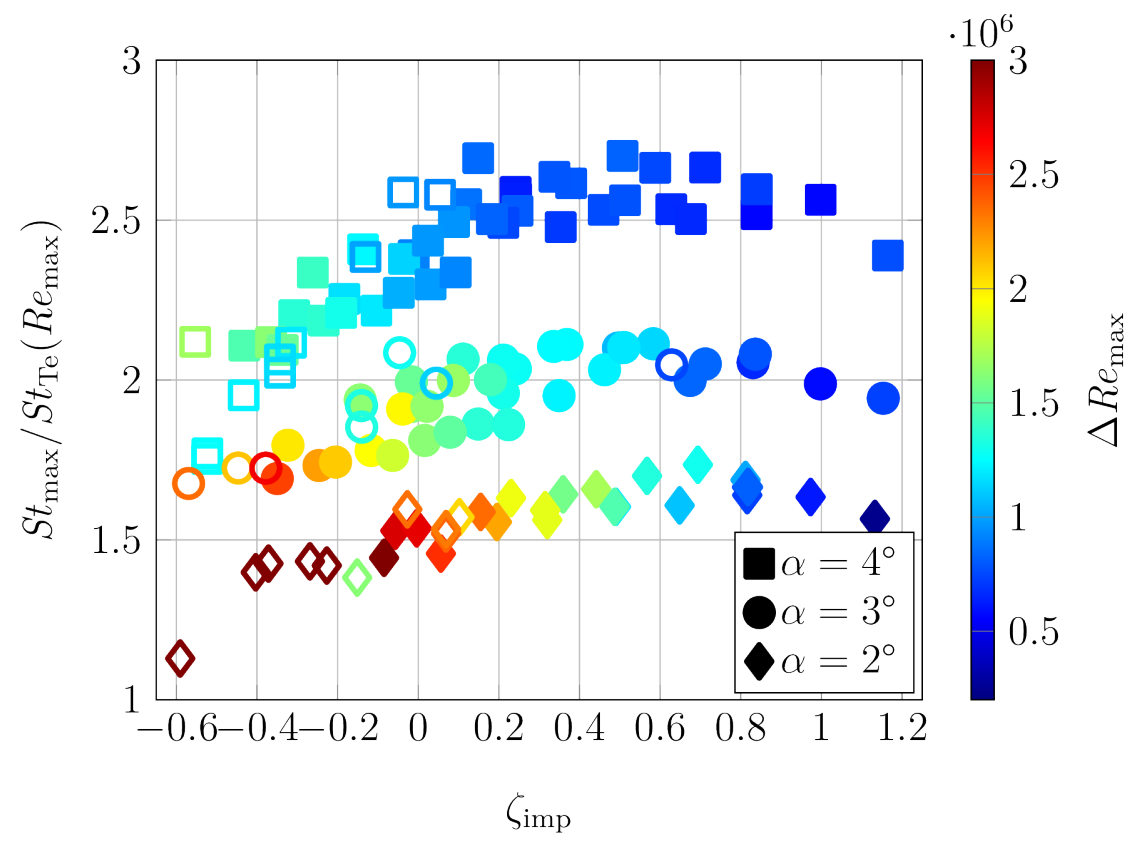

(a) Skalierte maximale Wärmestromdichte bei transitionellen SGWW für unterschiedliche Stoßintensitäten und $R e_{1}$. Die Datenpunkte sind anhand $\Delta R e_{\max }$ eingefärbt.

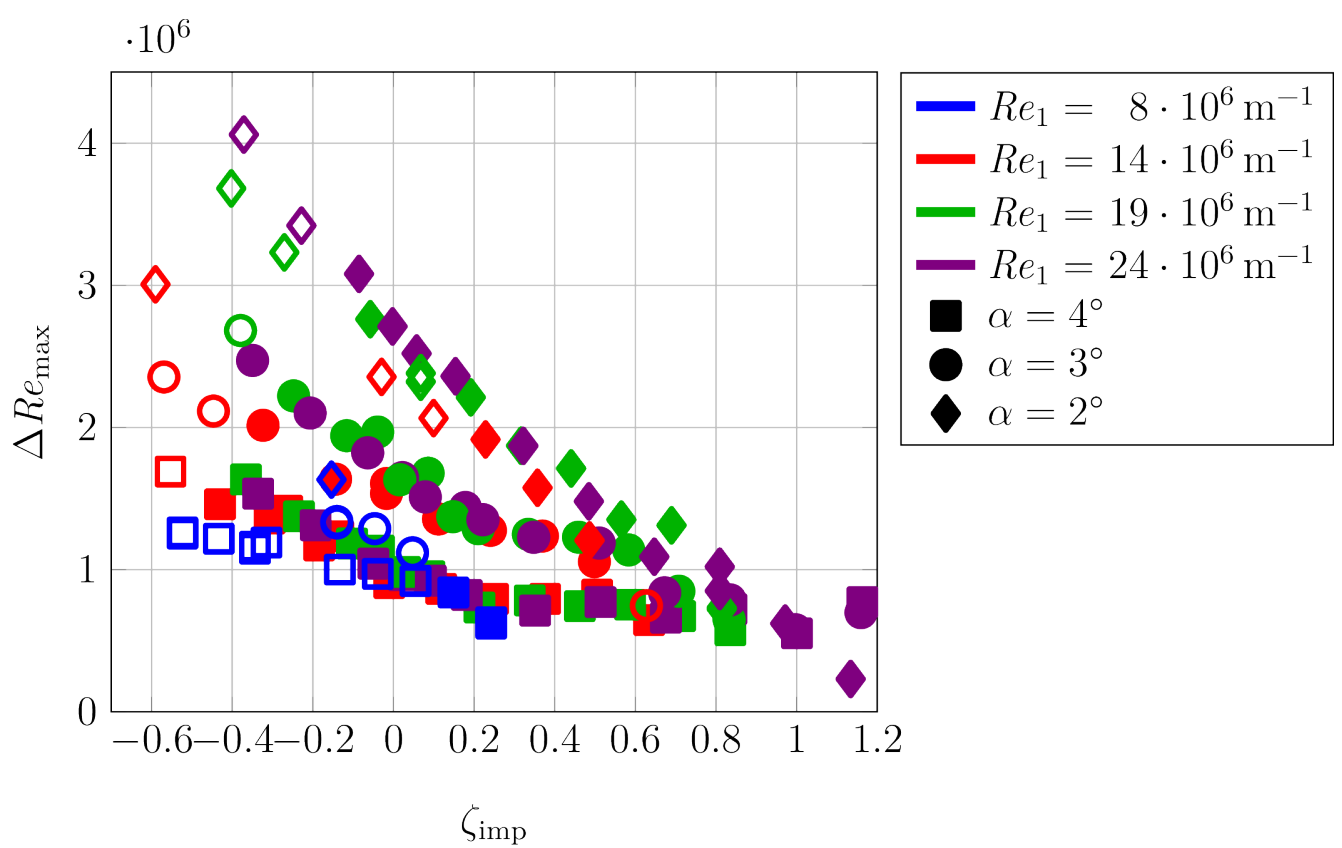

(b) $\Delta R e_{\max }$ in Abhängigkeit von $\zeta_{\text {imp }}$ für die untersuchten $R e_{1}$ und Stoßintensitäten.

Abbildung 4.34: Zusammenhang zwischen $\zeta_{\text {imp }}$, der Distanz bis zum Erreichen des Transitionsendes und die Auswirkung auf die stoßinduzierte Wärmestromdichte. 
ner ebenen Platte mit scharfer Vorderkante vergleichbare Messungen zu transitionellen SGWW durchgeführt wie in der aktuellen Studie. Der um eine Größenordnung kleinere Vorderkantenradius des damals eingesetzten Modells führte bei einer konstanten $R e_{1}$ zu einer Stromaufverschiebung des Transitionsgebietes, wodurch sich auch die lokale Grenzschichtdicke bei den transitionellen SGWW veränderte. Die Messdaten aus diesen Arbeiten wurden mit der aktuellen Skalierungsmethode ausgewertet und sind zum Vergleich in Abbildung 4.35 aufgetragen. Zwischen den unterschiedlichen Vorderkanten und Messkampagnen kann kein signifikanter Unterschied festgestellt werden, was die Gültigkeit der vorgestellten Skalierungsmethode untermauert.

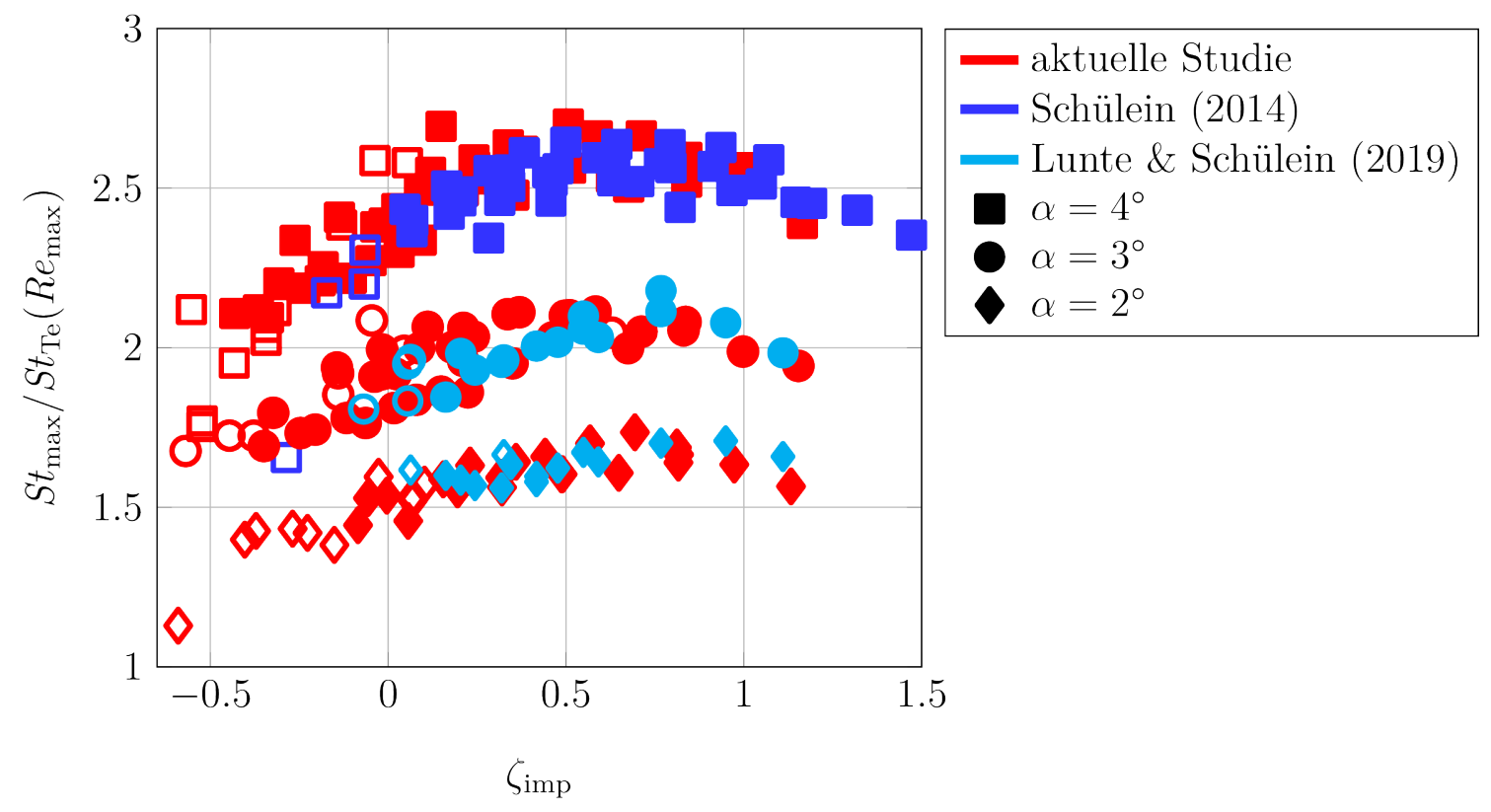

Abbildung 4.35: Vergleich der skalierten maximalen Wärmestromdichte bei transitionellen SGWW zwischen verschiedenen Messkampagnen mit unterschiedlichen Vorderkantenradien der ebenen Platte.

Die durchgeführten Vergleiche der Daten in Abb. 4.35 weisen eine einheitliche und deutliche Schichtung nach der Stoßintensität auf, die unabhängig von dem verwendeten Plattenmodell ist. Ein solcher Zusammenhang zwischen Stoßintensität und den skalierten Wärmelasten ist bereits seit längerem für turbulente SGWW bekannt. Holden (1977) gelang es eine zuverlässige empirische Gesetzmäßigkeit zu formulieren, die die skalierte maximale Wärmestromdichte mit dem Druckanstieg über das Interaktionsgebiet verknüpft:

$$
\frac{\dot{q}_{\max }}{\dot{q}_{1}}=\left(\frac{p_{\max }}{p_{1}}\right)^{0.85}
$$

Da im Rahmen dieser Studie keine statischen Druckmessungen entlang der Modelloberfläche durchgeführt wurden, wird der Druckanstieg über das Interaktionsgebiet durch 
das Verhältnis $p_{3} / p_{1}$ auf Basis der nicht-viskosen Stoßbeziehungen (Tabelle A.2) beschrieben. Werden die Verstärkungsfaktoren aus Abb. 4.35 zusätzlich mit $\left(p_{3} / p_{1}\right)^{0.85}$ skaliert, so entfällt in Abb. 4.36 die Schichtung nach der Stoßintensität und es liegt ein einheitlicher Verlauf der Datenpunkte vor. Der einheitliche Verlauf der Datenpunkte demonstriert, dass im Rahmen der untersuchten Parameterstudie die skalierten maximalen Wärmestromdichten bei transitionellen und turbulenten SGWW die selbe Skalierung bezüglich der Stoßintensität aufweisen. Die Verteilung der insgesamt 196 Datenpunkte kann durch eine Parabel mit einem Bestimmtheitsmaß von $R^{2}=0.707$ approximiert werden kann.

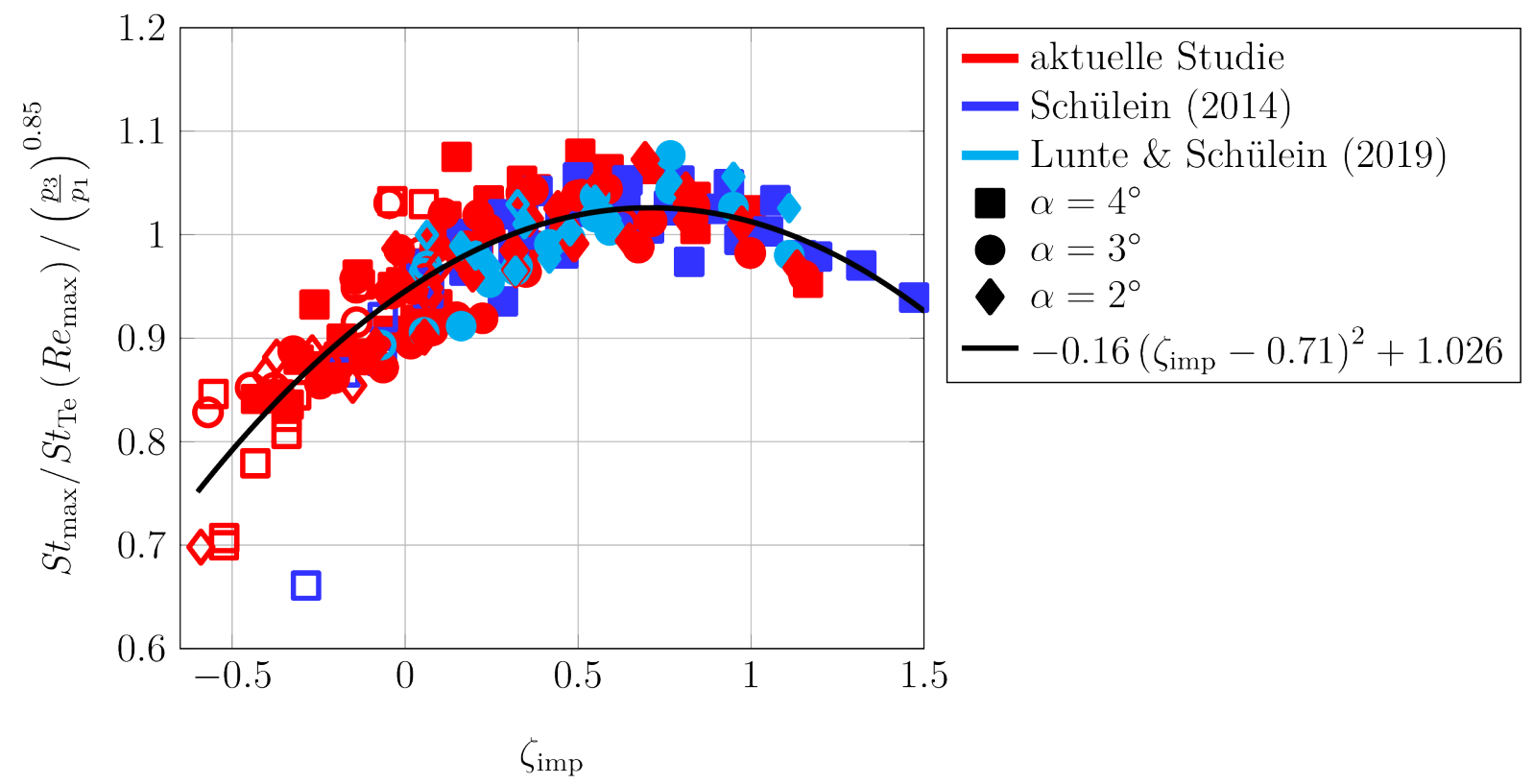

Abbildung 4.36: Durch eine zusätzliche Skalierung der Stoßintensität nach Holden (1977) ergibt sich ein einheitlicher Kurvenverlauf über $\zeta_{\text {imp }}$ für die skalierten maximalen Wärmestromdichten bei transitionellen SGWW.

Eine entscheidende Rolle bei der Skalierung der stoßinduzierten Wärmelasten spielt die Wahl der Referenz-Stantonzahl. Es ist bekannt, dass der Verlauf der Transition von den äußeren Einflüssen wie z.B. dem Turbulenzgrad der Außenströmung und der Beschaffenheit des Modells abhängt. Der einfallende Stoß führt zu einer Beschleunigung des Transitionsprozesses, da sowohl bestehende Instabilitäten verstärkt als auch neue Instabilitäten erzeugt werden (Pagella et al. 2002). Passieren Turbulenzflecken das Interaktionsgebiet, so vergrößern sich durch die SGWW die Ausmaße des Fleckens (Krishnan \& Sandham 2007), was einer Beschleunigung des Transitionsprozesses entspricht. Obwohl der einfallende Stoß die natürlichen Transition signifikant beeinflusst, ist der Transitionsprozess nicht allein durch den Stoß vorgegeben. Ausschlaggebend ist die Entwicklung der veränderten Störungen innerhalb der Grenzschicht, wodurch auch alle äußeren Einflüsse relevant bleiben. Zusätzlich handelt es sich bei der Transition um einen 
dynamischen Prozess, der sich auch beim Erreichen des Transitionsendes noch nicht im Gleichgewicht befindet (Holden 1977). Als Konsequenz misslingt eine einheitliche Skalierung der $S t_{\max }$-Werte auf Basis von rein theoretischen Referenzwerten, wie in Abb. $4.37 \mathrm{zu}$ erkennen ist. Durch die Verwendung des St-Niveaus am Transitionsende einer ungestörten Grenzschicht können diese ungreifbaren und situationsabhängigen Effekte zumindest rudimentär berücksichtigt werden. Um die erzielte Verbesserung bei der Skalierung durch die Verwendung von $S t_{\mathrm{Te}}\left(R e_{\mathrm{imp}}\right)$ aufzuzeigen, wurde in Abb. 4.37 als Referenzwert die theoretische $S t$-Verteilung für eine vollturbulente Grenzschicht in Form der Van Driest II Kurve (vgl. Kapitel 2.1.2) verwendet. Im Vergleich zu Abb. 4.36 streuen die Datenpunkte sichtbar stärker und lassen keinen gemeinsamen Verlauf erkennen. Bei der Van Driest II Kurve (Gl. 2.18 mit Gl. 2.15) wird eine turbulente Grenzschicht vom Beginn der Vorderkante angenommen. Hierdurch ist die Lauflänge der turbulenten Grenzschicht per se überschätzt und die St-Werte werden unterschätzt. Als Konsequenz fallen die normierten maximalen Wärmelasten in Abb. 4.37 höher aus als in Abb. 4.36,

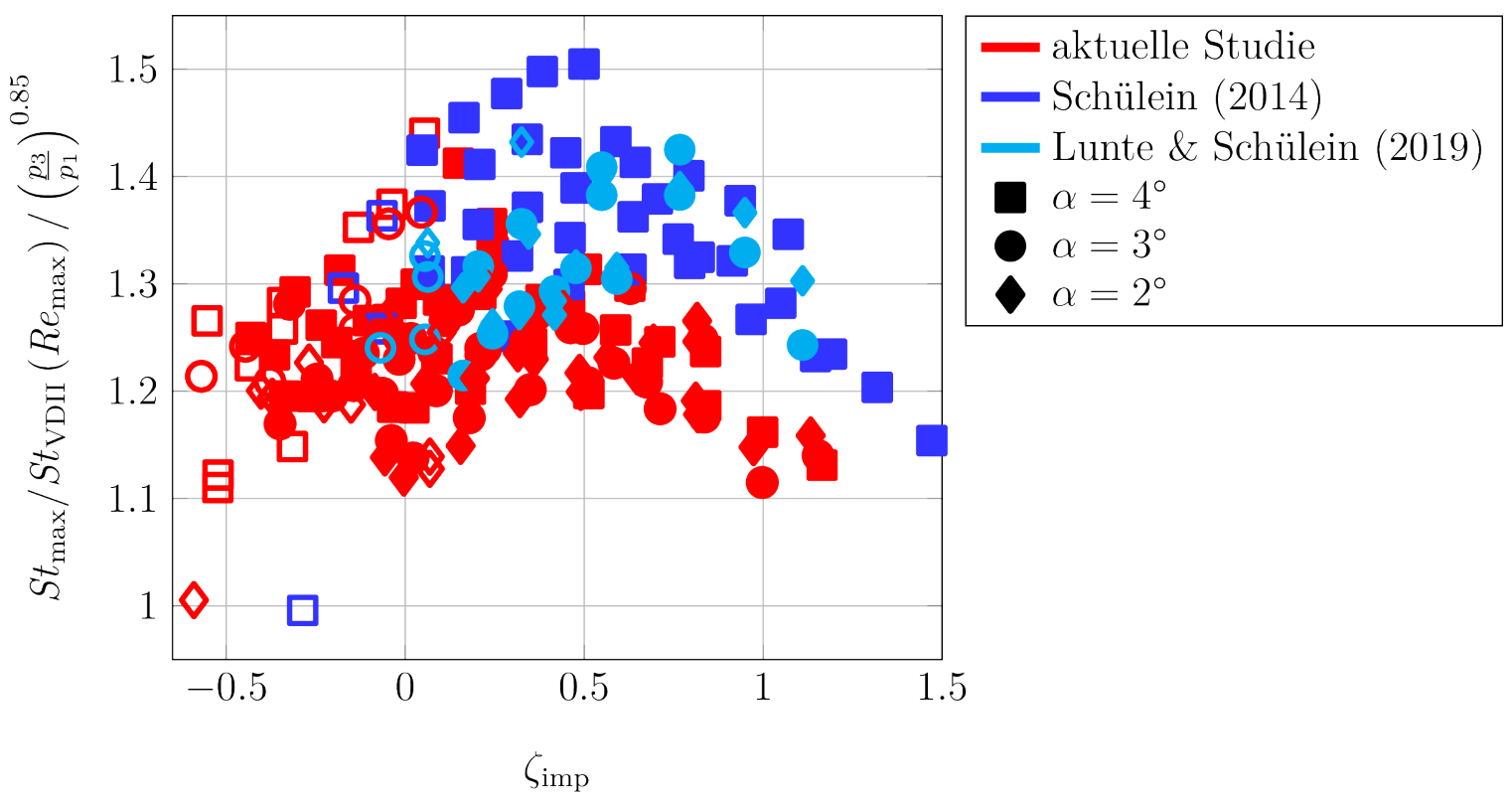

Abbildung 4.37: Bei der Verwendung eines theoretischen Referenzwertes in Form der Van Driest II Abschätzung kann der Einfluss der expliziten Strömungssituation auf die stoßinduzierten Wärmestromdichte nicht adäquat nachgebildet werden, wodurch kein einheitlicher Kurvenverlauf entsteht.

Obwohl in Abb.4.36 eine einheitliche Darstellung bezüglich der normierten maximalen Wärmelast gefunden wurde, kann die verwendete Skalierung nicht direkt zur Prognose der stoßinduzierten Wärmelasten genutzt werden. Die Invertierung des Skalierungsgesetzes scheitert an den verwendeten Referenzgrößen, die a priori nicht bekannt sind. Insbesondere $R e_{\max }$ kann erst im Versuch oder in einer Simulation adäquat ermittelt werden, wodurch sich die Prognose erübrigt. Um dieses Problem zu umgehen, können bei der Skalierung die $S t_{\mathrm{Te}}$-Werte auch an der Stoßauftreffposition ausgewertet werden. Auch für diesen abgewandelten Skalierungsansatz ergibt sich aus den Datenpunkten ein 
einheitlicher Kurvenverlauf, wie in Abb. 4.38 dargestellt, der durch eine Parabel mit einem Bestimmtheitsmaß von $R^{2}=0.797$ approximiert werden kann. Abbildung 4.34b zeigt, dass von der Anpassung des Skalierungsansatzes vor allem die kleineren Stoßintensitäten und die SGWW bei niedrigen $\zeta_{\text {imp }}$-Werten betroffen sind. Als Konsequenz kann in Abb. 4.38 eine stärkere Abhängigkeit der skalierten $S t_{\max }$ bezüglich $\zeta_{\text {imp }}$ beobachtet werden.



Abbildung 4.38: Die Verwendung von $S t_{\mathrm{Te}}\left(R e_{\mathrm{imp}}\right)$ als Referenzgröße ergibt auch einen einheitlichen Kurvenverlauf, der auch für eine Prognose der stoßinduzierten Wärmelasten bei transitionellen SGWW verwendet werden kann.

Für viele Anwendungsfälle genügt es die stoßinduzierten Wärmelasten bei transitionellen SGWW für das Worst-Case-Szenario abschätzen zu können. Nach Abb. 4.38 betragen die höchsten Normierungswerte circa 1. Für die Umrechnung vom Normierungswert zu $S t_{\max }$ kann der Druckanstieg $p_{3} / p_{1}$ entweder analytisch oder durch eine numerische Euler-Rechnung aus der Modellgeometrie gewonnen werden. Als letzte Skalierungsgröße verbleibt $S t_{\mathrm{Te}}\left(R e_{\mathrm{imp}}\right)$. Lohmeyer \& DeJarnette (2012) präsentierten eine Methode, mit der für jede $R e_{x}$-Position die hypothetische Wärmestromdichte des natürlichen Transitionsendes abgeschätzt werden kann. Unter der Annahme, dass am Transitionsende die Impulsverlustdicke der laminaren und turbulenten Grenzschicht identisch ist $\left(\delta_{2 \text {,lami }}=\delta_{2, \text { turb }}\right)$, kann ein effektiver Ursprung für die vollturbulente $S t$-Verteilung abgeleitet werden. Die Methode von Lohmeyer \& DeJarnette (2012) konnte die experimentellen $S t_{\mathrm{Te}}$-Werte dieser Arbeit mit einer durchschnittlichen Abweichung von $7 \%$ vorhersagen. Für diese Überprüfung wurde die laminare Impulsverlustdicke nach Gl.2.14 und die turbulente St-Verteilung nach Van Driest II (Gl. 2.18) verwendet. Eine Invertierung der Korrelation von Spalding \& Chi (1964) anhand der $c_{f}$-Verteilung nach Van 
Driest II (Gl. 2.18) ermöglicht die Abschätzung der turbulenten Impulsverlustdicke. Bei diesem Vorgehen können die höchsten stoßinduzierten Wärmelasten ohne experimentelle Vortests oder aufwendige Simulationen vorhergesagt werden.

Die Genauigkeit der Prognose kann weiter gesteigert werden, falls die St-Verteilung der ungestörten Transition bekannt ist. Zum einen entfällt die Abschätzung des St-Niveaus am ungestörten Transitionsende. Zum anderen ermöglichen die zusätzlichen Informationen bei einer gegebenen Stoßauftreffposition die Berechnung von $\zeta_{\text {imp }}$, wodurch aus Abb. 4.38 der Normierungswert abgelesen werden kann. Bislang kann die St-Verteilung der ungestörten Transition nur mittels Experimenten unter identischen Strömungsbedingungen zum Anwendungsfall verlässlich bestimmt werden. Aber zukünftige Fortschritte beim Verständnis der natürlichen Transition und deren Vorhersage mit z. B. Direkter Numerischer Simulation, könnten eine verbesserte Prognose von $S t_{\max }$ ohne Experiment ermöglichen. 


\section{Zusammenfassung}

Die Interaktion eines einfallenden Verdichtungsstoßes mit der transitionellen Grenzschicht auf einer ebenen Platte wurde experimentell untersucht. Die Parameterstudie wurde bei Mach 6 im Rohrwindkanal Göttingen durchgeführt und umfasst eine $R e_{1^{-}}$ Variation zwischen $8 \cdot 10^{6} \mathrm{~m}^{-1}$ und $24 \cdot 10^{6} \mathrm{~m}^{-1}$, drei Stoßintensitäten $\alpha=2^{\circ}, 3^{\circ}$ und $4^{\circ}$ sowie die unabhängige Variation der Stoßauftreffstelle und der natürlichen Transitionslage. Im Mittelpunkt der Experimente stand die Untersuchung der räumlichen StVerteilung, aber auch Schattenaufnahmen wurden zur Visualisierung der Strömung angefertigt. Zusätzlich wurden mittels zeitaufgelösten PCB- und FLDI-Messungen die Druckund Dichteschwankungen der transitionellen Grenzschicht untersucht und zur Analyse des Grenzschichtzustandes verwendet. Die wesentlichen Erkenntnisse der experimentellen Studie sollen im Folgenden zusammengefasst werden.

Da die FLDI erstmalig am RWG eingesetzt wurde und die Montage der PCB-Sensoren hinsichtlich der Schwingungsentkopplung angepasst werden musste, wurden die Eigenschaften beider Messtechniken detailliert untersucht. Hierbei wurde unter anderem aufgezeigt, dass die messbare obere Grenzfrequenz bezüglich der Druck- und Dichteschwankungen nicht ausreicht um das Frequenzspektrum von turbulenten Grenzschichten vollständig zu messen. Eine $-3 \mathrm{~dB}$ Dämpfung im Leistungsdichtespektrum liegt bei den Druckschwankungen für $f=185 \mathrm{kHz}$ vor und bei den Dichteschwankungen für $f=2.2 \mathrm{MHz}$. Trotz der eingeschränkten Messeigenschaften konnten Instabilitäten und Turbulenzflecken innerhalb der transitionellen Grenzschicht detektiert werden. Ein Vergleich mit etablierten Korrelationen bestätigte, dass es sich bei den Instabilitäten um zweite Mack-Moden handelte, allerdings wurde eine verbleibende $R e_{1}$-Abhängigkeit bei den skalierten Frequenzen festgestellt. Der $R e_{1}$-Effekt konnte aufgelöst werden, indem bei der Skalierung als Längenmaß der wandnormale Abstand des Wendepunktes der akustischen Feldimpedanz anstelle der Grenzschichtdicke verwendet wurde. Dieser Wendepunkt scheint mit dem Einfangmechanismus der zweiten Mack-Moden innerhalb der Grenzschicht deutlich besser zu korrelieren (Kuehl 2018), wodurch sich eine Verbesserung hinsichtlich der Korrelation ergibt.

Mit einem eigens entwickelten Auswerteverfahren konnten die Turbulenzflecken in den Messsignalen der Druck- und Dichteschwankungen identifiziert und zur Beschreibung des Transitionsfortschritts auf Grundlage des Intermittenzwertes verwendet werden. Ein Vergleich diverser Methoden zur Intermittenzbestimmung demonstrierte, wie konsistent die Transitionslage anhand unterschiedlicher Strömungsgrößen bestimmt werden kann. Die Abweichungen zwischen den Kurven entstehen zum Teil auch durch die messtechnikbedingten Veränderungen am Modell. Es konnte demonstriert werden, dass der messbare Intermittenzverlauf sämtlicher Verfahren im Einklang mit der bekannten universellen Intermittenzentwicklung ist. 


\section{Zusammenfassung}

Für die Untersuchung der SGWW wurde zunächst die Auftreffposition $x_{\text {imp }}$ des einfallenden Stoßes anhand von Schattenaufnahmen bestimmt. Die Analyse der Einzelaufnahmen ergab, dass die Auftreffposition während einer Messung um $\sigma_{x_{\text {imp }}}=1.4 \mathrm{~mm}$ fluktuierte. Weiterhin wurde nachgewiesen, dass die Auswertung eines gemittelten Schattenbildes zum selben $x_{\text {imp }}$-Wert führt wie der statistische Mittelwert aus den Einzelbildern, sodass die geringe zeitliche Varianz der Auftreffposition innerhalb eines Testpunktes keine negativen Auswirkungen auf die weiterführenden Untersuchungen hat.

Durch die Analyse der St-Verläufe und der bestimmten Intermittenzverteilungen konnte erstmalig experimentell bestätigt werden, dass die maximalen stoßinduzierten Wärmestromdichten $S t_{\max }$ bei transitionellen SGWW an der Position des neuen Transitionsendes entstehen. Der räumliche Abstand zwischen der Stoßauftreffstelle und dem Transitionsende ergibt sich aus der Anfälligkeit der Grenzschicht gegenüber den stoßinduzierten Störungen. Bei ausreichender Stoßintensität wird der Transitionsprozess unmittelbar ausgelöst und signifikant beschleunigt. Sind die stoßinduzierten Störungen hingegen unzureichend, bleibt die Grenzschicht auch stromab von $x_{\text {imp }}$ zunächst laminar. In allen untersuchten Fällen verschob sich das Transitionsende mit einer SGWW stromauf gegenüber dem ungestörten Fall.

Die Entwicklung der Transition stromab von $x_{\text {imp }}$ beeinflusst auch die maximale stoBinduzierte Wärmestromdichte. Zu den identifizierten Einflussfaktoren auf die Höhe von $S t_{\max }$ zählen die Stoßintensität, die lokale Grenzschichtdicke, der Transitionsfortschritt an der Stoßauftreffstelle und das St-Niveau am Transitionsende des ungestörten Referenzfalls. Durch eine quantitative Berücksichtigung jedes Einflussfaktors konnte ein Skalierungsansatz vorgestellt werden, der alle vorhandenen und neu gewonnenen experimentellen Daten zur maximalen Wärmelast infolge von SGWW in transitionellen Grenzschichten in einer gemeinsamen Gesetzmäßigkeit abbilden lässt. Bei der Ausarbeitung des Skalierungsanssatzes erwies sich der Intermittenzwert zur Quantifizierung des Grenzschichtzustandes an der Stoßauftreffstelle als ungeeignet, da der Intermittenzwert keine Charakterisierung der Grenzschicht außerhalb des eigentlichen Transitionsgebietes ermöglicht. Stattdessen wurde die Längskoordinate $\zeta$ verwendet, die mit der Ausdehnung des Transitionsbereichs normiert ist und deren Ursprung am Transitionsbeginn liegt. Die durchgehende Einsortierung des Grenzschichtzustandes auch außerhalb des Transitionsgebietes ist der entscheidende Vorteil von $\zeta$.

Ausgehend von dem Ergebnis des Skalierungsansatzes wurde eine Methode erläutert, um die maximalen Wärmelasten bei transitionellen SGWW zu prognostizieren. Da die Vorhersage auch ohne aufwendige Simulationen oder experimentelle Voruntersuchungen durchführbar ist, kann die Methode bereits in der Vorentwurfsphase von Überschallfluggeräten eingesetzt werden. 


\section{A Anhang}

\section{A.1 Herleitung der Gleichungen zur kompressiblen laminaren Grenzschicht}

In diesem Abschnitt wird vorgeführt, wie man zu den Gleichungen 2.10 bis 2.14 aus Kapitel 2.1.1 gelangt. Zunächst werden zur Vorbereitung einige Zwischenrechnungen durchgeführt. Nach Gleichung 2.4 folgt

$$
\frac{\partial \eta}{\partial y}=\rho \sqrt{\frac{u_{\infty}}{2 \rho_{\infty} \mu_{\infty} x}}=\frac{\rho u_{\infty}}{\mu_{\infty}} \sqrt{\frac{\mu_{\infty}}{2 \rho_{\infty} u_{\infty} x}}=\frac{\rho u_{\infty}}{\mu_{\infty}} \frac{1}{\sqrt{2 R e_{x}}} .
$$

Ferner gilt

$$
\begin{aligned}
g^{\prime} & =\frac{\partial g}{\partial \eta}=\frac{1}{T_{\infty}} \frac{\partial T}{\partial \eta}=\frac{1}{T_{\infty}} \frac{\partial T}{\partial y} \frac{\partial y}{\partial \eta}=\frac{\partial T}{\partial y} \frac{\mu_{\infty} \sqrt{2 R e_{x}}}{\rho u_{\infty} T_{\infty}} \\
\Longleftrightarrow \frac{\partial T}{\partial y} & =\frac{\rho u_{\infty} T_{\infty} g^{\prime}}{\mu_{\infty} \sqrt{2 R e_{x}}}
\end{aligned}
$$

und

$$
\begin{aligned}
f^{\prime \prime} & =\frac{\partial f^{\prime}}{\partial \eta}=\frac{1}{u_{\infty}} \frac{\partial u}{\partial y} \frac{\partial y}{\partial \eta}=\frac{\partial u}{\partial y} \frac{\mu_{\infty} \sqrt{2 R e_{x}}}{\rho u_{\infty}^{2}} \\
\Longleftrightarrow \frac{\partial u}{\partial y} & =\frac{\rho u_{\infty}^{2} f^{\prime \prime}}{\mu_{\infty} \sqrt{2 R e_{x}}} .
\end{aligned}
$$

Nach Schlichting \& Gersten (2006) kann die Wärmestromdichte mittels

$$
\dot{q}_{\mathrm{w}}=-\left.\lambda \frac{\partial T}{\partial y}\right|_{y=0}
$$

berechnet werden, wobei $\lambda$ die Wärmeleitfähigkeit des Mediums angibt. Nach Definition der Prandtl-Zahl gilt

$$
\operatorname{Pr}=\frac{\mu c_{p}}{\lambda}
$$

Dabei ist $c_{p}$ die spezifische Wärmekapazität. Ausgehend von der Definition der Stanton- 
Zahl (Schlichting \& Gersten 2006) folgt

$$
\begin{aligned}
S t & =\frac{\dot{q}_{\mathrm{w}}}{\rho_{\infty} u_{\infty} c_{p}\left(T_{\mathrm{w}}-T_{\mathrm{aw}}\right)}=\left.\frac{-\lambda}{\rho_{\infty} u_{\infty} c_{p}\left(T_{\mathrm{w}}-T_{\mathrm{aw}}\right)} \frac{\partial T}{\partial y}\right|_{y=0} \\
& =\left.\frac{\lambda}{\rho_{\infty} u_{\infty} c_{p}\left(T_{\mathrm{aw}}-T_{\mathrm{w}}\right)} \frac{\rho u_{\infty} T_{\infty} g^{\prime}}{\mu_{\infty} \sqrt{2 R e_{x}}}\right|_{\eta=0}=\left.\frac{T_{\infty} g^{\prime}}{\left(T_{\mathrm{aw}}-T_{\mathrm{w}}\right) \sqrt{2 R e_{x}}} \frac{\rho \lambda}{\rho_{\infty} \mu_{\infty} c_{p}}\right|_{\eta=0} \\
& =\left.\frac{T_{\infty} g^{\prime}}{\operatorname{Pr}\left(T_{\mathrm{aw}}-T_{\mathrm{w}}\right) \sqrt{2 R e_{x}}} \frac{\rho \mu}{\rho_{\infty} \mu_{\infty}}\right|_{\eta=0}=\frac{T_{\infty} g^{\prime}(0)}{\operatorname{Pr}\left(T_{\mathrm{aw}}-T_{\mathrm{w}}\right) \sqrt{2 R e_{x}}} \frac{\rho_{\mathrm{w}} \mu_{\mathrm{w}}}{\rho_{\infty} \mu_{\infty}} .
\end{aligned}
$$

Die Wandschubspannung $\tau_{\mathrm{w}}$ (Schlichting \& Gersten 2006) kann durch

$$
\tau_{\mathrm{w}}=\left.\mu \frac{\partial u}{\partial y}\right|_{y=0}
$$

berechnet werden, sodass für den Reibungsbeiwert (Schlichting \& Gersten 2006) gilt

$$
\begin{aligned}
c_{f} & =\frac{2 \tau_{\mathrm{w}}}{\rho_{\infty} u_{\infty}^{2}}=\left.\frac{2 \mu}{\rho_{\infty} u_{\infty}^{2}} \frac{\partial u}{\partial y}\right|_{y=0}=\left.\frac{2 \mu}{\rho_{\infty} u_{\infty}^{2}} \frac{\rho u_{\infty}^{2} f^{\prime \prime}}{\mu_{\infty} \sqrt{2 R e_{x}}}\right|_{\eta=0} \\
& =\left.\frac{\sqrt{2} f^{\prime \prime}}{\sqrt{R e_{x}}} \frac{\rho \mu}{\rho_{\infty} \mu_{\infty}}\right|_{\eta=0}=\frac{\sqrt{2} f^{\prime \prime}(0)}{\sqrt{R e_{x}}} \frac{\rho_{\mathrm{w}} \mu_{\mathrm{w}}}{\rho_{\infty} \mu_{\infty}} .
\end{aligned}
$$

Die Grenzschichtdicke $\delta_{99}$ ergibt sich für den wandnormalen Abstand, an dem $f^{\prime}=$ 0.99 erreicht wird. Der selbe Wandabstand wird in den Ähnlichkeitsvariablen als $\eta_{99}$ bezeichnet. Die Umrechnung erfolgt durch

$$
\begin{aligned}
\delta_{99} & =\int_{0}^{\delta_{99}} \mathrm{~d} y=\frac{\sqrt{2 R e_{x}} \mu_{\infty}}{u_{\infty}} \int_{0}^{\eta_{99}} \frac{1}{\rho} \mathrm{d} \eta=\frac{\sqrt{2} x}{\sqrt{R e_{x}}} \int_{0}^{\eta_{99}} \frac{\rho_{\infty}}{\rho} \mathrm{d} \eta \\
& =\frac{\sqrt{2} x}{\sqrt{R e_{x}}} \int_{0}^{\eta_{99}} \frac{T}{T_{\infty}} \mathrm{d} \eta=\frac{\sqrt{2} x}{\sqrt{R e_{x}}} \int_{0}^{\eta_{99}} g \mathrm{~d} \eta .
\end{aligned}
$$

Für die Verdrängungsdicke in einer kompressiblen Grenzschicht (White 1991) ergibt sich

$$
\begin{aligned}
\delta_{1} & =\int_{0}^{\infty} 1-\frac{\rho u}{\rho_{\infty} u_{\infty}} \mathrm{d} y=\frac{\mu_{\infty} \sqrt{2 R e_{x}}}{u_{\infty}} \int_{0}^{\infty} \frac{1}{\rho}-\frac{u}{\rho_{\infty} u_{\infty}} \mathrm{d} \eta \\
& =\frac{\mu_{\infty} \sqrt{2 R e_{x}}}{u_{\infty} \rho_{\infty}} \int_{0}^{\infty} \frac{\rho_{\infty}}{\rho}-\frac{u}{u_{\infty}} \mathrm{d} \eta=\sqrt{\frac{2 \mu_{\infty} x}{u_{\infty} \rho_{\infty}}} \int_{0}^{\infty} \frac{T}{T_{\infty}}-\frac{u}{u_{\infty}} \mathrm{d} \eta \\
& =\frac{\sqrt{2} x}{\sqrt{R e_{x}}} \int_{0}^{\infty} g-f^{\prime} \mathrm{d} \eta .
\end{aligned}
$$

Für die Impulsverlustdicke in einer kompressiblen Grenzschicht (White 1991) ergibt sich

$$
\begin{aligned}
\delta_{2} & =\int_{0}^{\infty} \frac{\rho u}{\rho_{\infty} u_{\infty}}\left(1-\frac{u}{u_{\infty}}\right) \mathrm{d} y=\frac{\mu_{\infty} \sqrt{2 R e_{x}}}{u_{\infty} \rho_{\infty}} \int_{0}^{\infty} \frac{u}{u_{\infty}}\left(1-\frac{u}{u_{\infty}}\right) \mathrm{d} \eta \\
& =\frac{\sqrt{2} x}{\sqrt{R e_{x}}} \int_{0}^{\infty} f^{\prime}\left(1-f^{\prime}\right) \mathrm{d} \eta .
\end{aligned}
$$




\section{A.2 Informationen bezüglich der PCB-Sensoren}

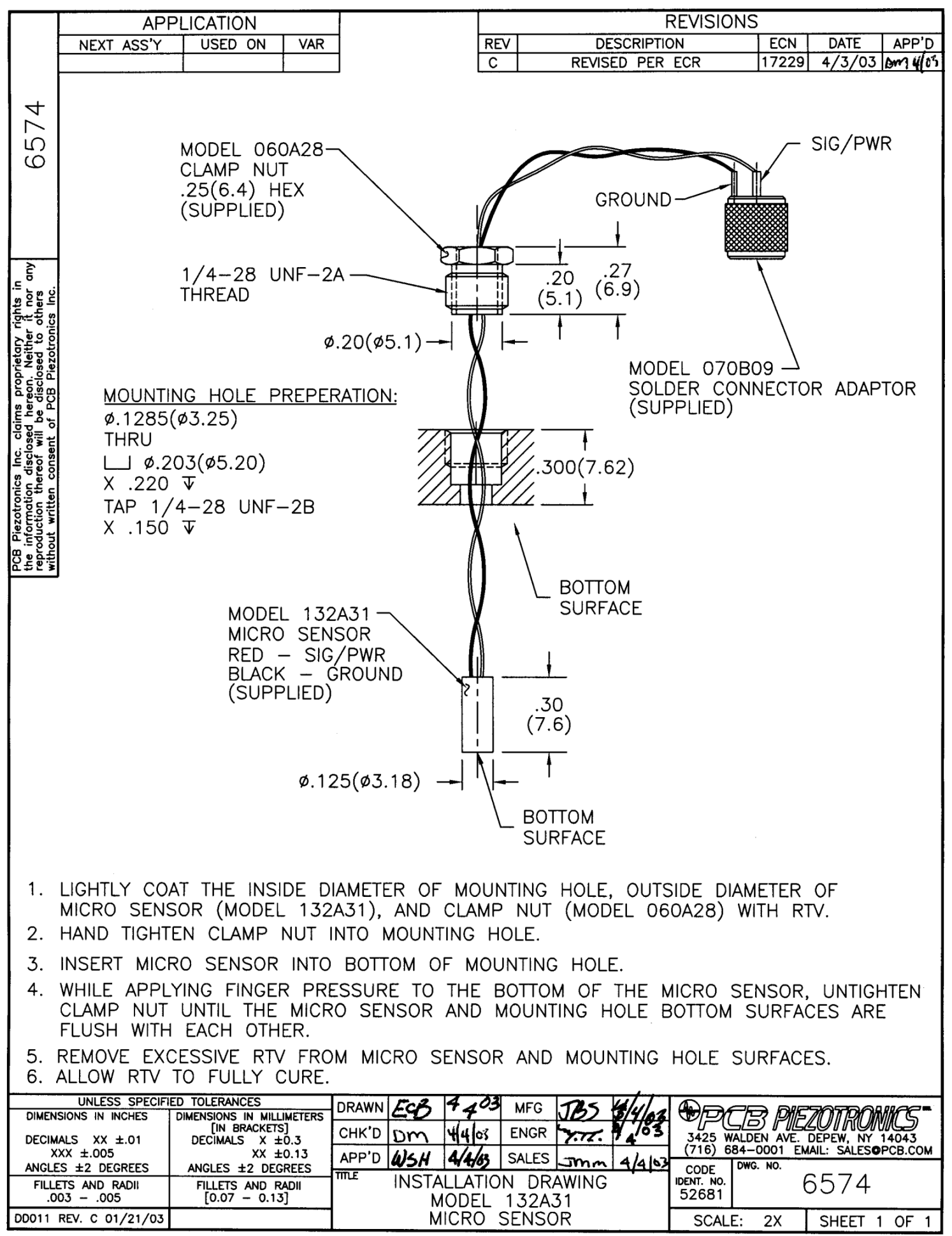

Abbildung A.1: Einbauanleitung der PCB-Sensoren 


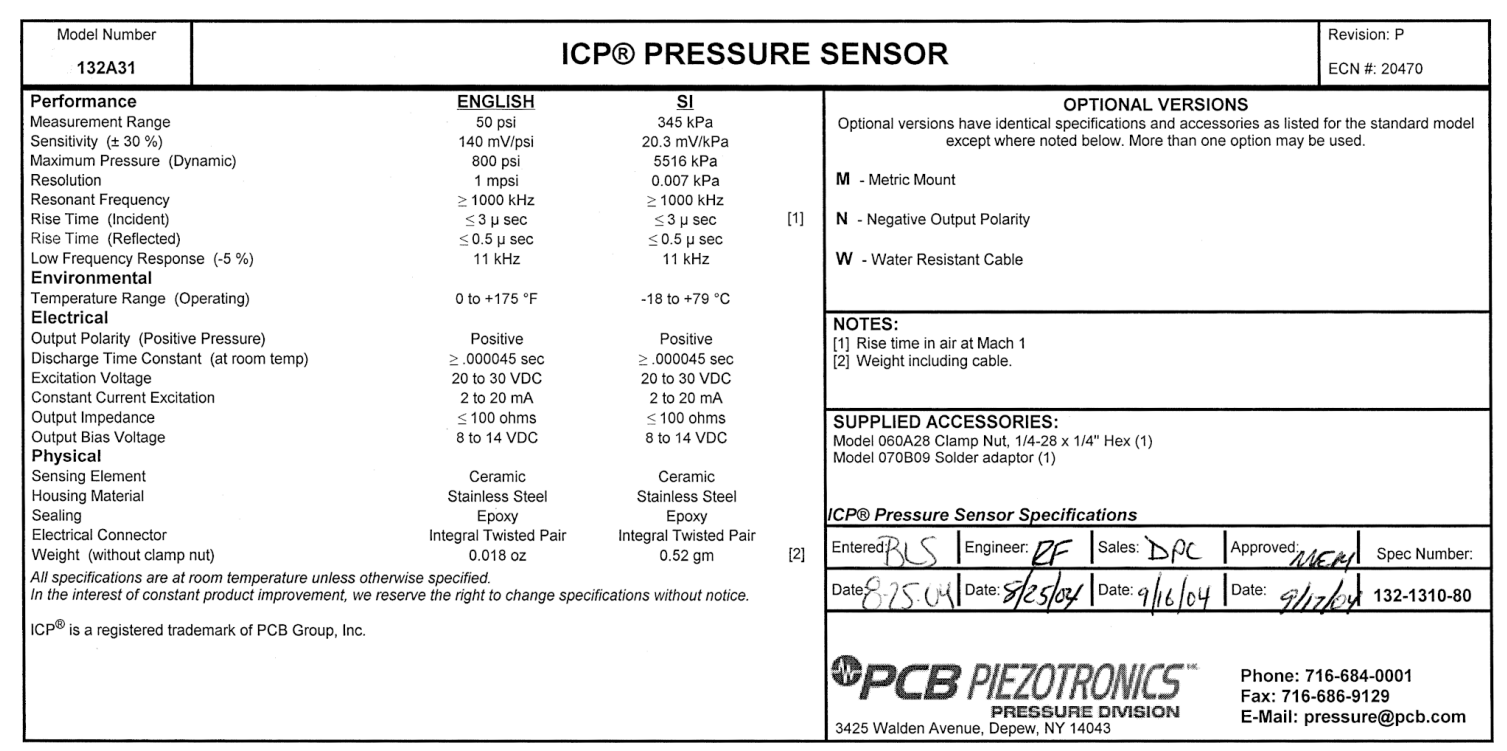

Abbildung A.2: Datenblatt der verwendeten $P C B$-132A31 Sensoren

\begin{tabular}{|c|c|c|c|c|}
\hline \multirow{2}{*}{$\begin{array}{l}\text { Einbau- } \\
\text { position } \\
\end{array}$} & \multirow{2}{*}{$\begin{array}{c}\text { Seriennr. } \\
\text { PCB-Sensor }\end{array}$} & \multirow{2}{*}{$\begin{array}{c}\text { Sensitivität } \\
{[\mu \mathrm{V} / \mathrm{Pa}]}\end{array}$} & \multicolumn{2}{|c|}{$(x, y)$ in $\mathrm{mm}$} \\
\hline & & & stromauf & stromab \\
\hline 1 & 7561 & 18.09 & $(106.00,-65.00)$ & $(486.00, \quad 65.00)$ \\
\hline 2 & 7560 & 21.20 & $(118.67,-56.33)$ & $(473.33, \quad 56.33)$ \\
\hline 3 & 7562 & 24.50 & $(131.33,-47.67)$ & $(460.67, \quad 47.67)$ \\
\hline 4 & 7563 & 20.74 & $(144.00,-39.00)$ & $(448.00, \quad 39.00)$ \\
\hline 5 & 7564 & 24.38 & $(156.67,-30.33)$ & $(435.33, \quad 30.33)$ \\
\hline 6 & 7566 & 21.33 & $(169.33,-21.67)$ & $(422.67, \quad 21.67)$ \\
\hline 7 & 7567 & 19.30 & $(182.00,-13.00)$ & $(410.00, \quad 13.00)$ \\
\hline 8 & 7568 & 20.49 & $(194.67,-4.33)$ & $(397.33, \quad 4.33)$ \\
\hline 9 & 7569 & 24.12 & $(207.33, \quad 4.33)$ & $(384.67,-4.33)$ \\
\hline 10 & 7590 & 17.85 & $(220.00, \quad 13.00)$ & $(372.00,-13.00)$ \\
\hline 11 & 7591 & 17.85 & $(232.67, \quad 21.67)$ & $(359.33,-21.67)$ \\
\hline 12 & 7592 & 19.54 & $(245.33, \quad 30.33)$ & $(346.67,-30.33)$ \\
\hline 13 & 7593 & 19.54 & $(258.00, \quad 39.00)$ & $(334.00,-39.00)$ \\
\hline 14 & 7594 & 22.92 & $(270.67, \quad 47.67)$ & $(321.33,-47.67)$ \\
\hline 15 & 7595 & 23.39 & $(283.33, \quad 56.33)$ & $(308.67,-56.33)$ \\
\hline 16 & 7596 & 17.91 & $(296.00, \quad 65.00)$ & $(296.00,-65.00)$ \\
\hline
\end{tabular}

Tabelle A.1: Zuordnung zwischen der Einbauposition bei der PCB-Einsatzplatte und dem verwendeten PCB-Sensor. Zu jeder Einbauposition sind die beiden Messpositionen im Koordinatensystem der ebenen Platte angegeben. 


\section{A.3 Tabellen zur Messmatrix}

\begin{tabular}{ccccc}
\hline Ablenkwinkel & Strömungsgröße & $\mathrm{G} 1$ & $\mathrm{G} 2$ & $\mathrm{G} 3$ \\
\hline $2^{\circ}$ & $M a$ & 5.98 & 5.70 & 5.44 \\
& $\beta$ & $10.94^{\circ}$ & $11.42^{\circ}$ & - \\
& $p / p_{1}$ & 1 & 1.34 & 1.76 \\
$\rho / \rho_{1}$ & 1 & 1.23 & 1.50 \\
$T / T_{1}$ & 1 & 1.09 & 1.18 \\
& $u / u_{1}$ & 1 & 0.994 & 0.988 \\
\hline $3^{\circ}$ & $M a$ & 5.98 & 5.57 & 5.20 \\
& $\beta$ & $11.65^{\circ}$ & $12.37^{\circ}$ & - \\
& $p / p_{1}$ & 1 & 1.54 & 2.29 \\
$\rho / \rho_{1}$ & 1 & 1.36 & 1.80 \\
$T / T_{1}$ & 1 & 1.13 & 1.27 \\
& $u / u_{1}$ & 1 & 0.991 & 0.981 \\
\hline $4^{\circ}$ & $M a$ & 5.98 & 5.43 & 4.97 \\
& $\beta$ & $12.40^{\circ}$ & $13.38^{\circ}$ & - \\
& $p / p_{1}$ & 1 & 1.76 & 2.95 \\
& $\rho / \rho_{1}$ & 1 & 1.49 & 2.14 \\
& $T / T_{1}$ & 1 & 1.18 & 1.37 \\
$u / u_{1}$ & 1 & 0.987 & 0.973 \\
\hline
\end{tabular}

Tabelle A.2: Strömungsparameter stromab des einfallenden (G2) und reflektierten (G3) Stoßes. Die Strömungsgrößen $p, \rho, T$ und $u$ sind mit den Anströmungsbedingungen (G1) skaliert.

\begin{tabular}{|c|c|c|c|}
\hline $\begin{array}{l}\text { Stoßgen.- } \\
\text { Position }\end{array}$ & $\alpha$ & $R e_{1}\left[10^{6} \mathrm{~m}^{-1}\right]$ & Messtechnik \\
\hline ohne & - & $8,14,19,24$ & QIRT, PCB, FLDI \\
\hline Pos. III & $2^{\circ}, 3^{\circ}, 4^{\circ}$ & $8,14,19,24$ & QIRT, PCB \\
\hline Pos. V & $2^{\circ}, 3^{\circ}, 4^{\circ}$ & $8,14,19,24$ & QIRT, PCB \\
\hline \multirow[t]{2}{*}{ Pos. VII } & $2^{\circ}, 3^{\circ}, 4^{\circ}$ & $8,14,19,24$ & QIRT, PCB \\
\hline & $3^{\circ}$ & $8,14,19,24$ & FLDI \\
\hline Pos. IX & $2^{\circ}, 3^{\circ}, 4^{\circ}$ & $8,14,19,24$ & QIRT \\
\hline Pos. XI & $3^{\circ}$ & $8,14,19,24$ & QIRT \\
\hline
\end{tabular}

Tabelle A.3: Übersicht zur Messmatrix bei Pos. A 


\begin{tabular}{|c|c|c|c|}
\hline $\begin{array}{l}\text { Stoßgen.- } \\
\text { Position }\end{array}$ & $\alpha$ & $\operatorname{Re}_{1}\left[10^{6} \mathrm{~m}^{-1}\right]$ & Messtechnik \\
\hline ohne & - & $8,14,19,24$ & QIRT, PCB \\
\hline Pos. V & $2^{\circ}, 3^{\circ}, 4^{\circ}$ & $8,14,19,24$ & QIRT \\
\hline Pos. VII & $2^{\circ}, 3^{\circ}, 4^{\circ}$ & $8,14,19,24$ & QIRT \\
\hline Pos. IX & $2^{\circ}, 3^{\circ}, 4^{\circ}$ & $8,14,19,24$ & QIRT \\
\hline \multirow[t]{2}{*}{ Pos. XI } & $2^{\circ}$ & $14,19,24$ & QIRT \\
\hline & $3^{\circ}, 4^{\circ}$ & $8,14,19,24$ & QIRT \\
\hline Pos. XIII & $2^{\circ}, 3^{\circ}, 4^{\circ}$ & $8,14,19,24$ & QIRT, PCB \\
\hline Pos. XV & $2^{\circ}, 3^{\circ}, 4^{\circ}$ & $8,14,19,24$ & QIRT \\
\hline \multirow[t]{2}{*}{ Pos. XVII } & $2^{\circ}, 3^{\circ}$ & $14,19,24$ & QIRT \\
\hline & $4^{\circ}$ & $8,14,19,24$ & QIRT \\
\hline Pos. XIX & $2^{\circ}, 3^{\circ}, 4^{\circ}$ & $8,14,19,24$ & QIRT, PCB \\
\hline
\end{tabular}

Tabelle A.4: Übersicht zur Messmatrix bei Pos. B. Der Stoßgenerator wurde um $10 \mathrm{~mm}$ erhöht.

\begin{tabular}{lccc}
\hline $\begin{array}{l}\text { Stoßgen.- } \\
\text { Position }\end{array}$ & $\alpha$ & $R e_{1}\left[10^{6} \mathrm{~m}^{-1}\right]$ & $\begin{array}{c}\text { Stoßgen.- } \\
\text { Verschiebung }\end{array}$ \\
\hline ohne & - & $8,14,19,24$ & - \\
Pos. IX & $2^{\circ}, 3^{\circ}, 4^{\circ}$ & $8,14,19,24$ & $0 \mathrm{~mm}$ \\
Pos. XIII & $2^{\circ}, 3^{\circ}, 4^{\circ}$ & $8,14,19,24$ & $10 \mathrm{~mm}$ \\
\hline
\end{tabular}

Tabelle A.5: Übersicht zur Messmatrix der Schattenaufnahmen

\section{A.4 Werte zur Intermittenzbestimmung}




\begin{tabular}{lcc}
\hline$R e_{1}\left[10^{6} \mathrm{~m}^{-1}\right]$ & $\left(R e_{\mathrm{Tb}}, S t_{\mathrm{Tb}}\right)$ & $\left(R e_{\mathrm{Te}}, S t_{\mathrm{Te}}\right)$ \\
\hline 8 & $\left(2.65 \cdot 10^{6}, 2.54 \cdot 10^{-4}\right)$ & $\left(5.32 \cdot 10^{6},-\right)$ \\
14 & $\left(4.07 \cdot 10^{6}, 2.27 \cdot 10^{-4}\right)$ & $\left(7.49 \cdot 10^{6},-\right)$ \\
19 & $\left(4.57 \cdot 10^{6}, 2.10 \cdot 10^{-4}\right)$ & $\left(8.90 \cdot 10^{6}, 10.11 \cdot 10^{-4}\right)$ \\
24 & $\left(5.47 \cdot 10^{6}, 1.97 \cdot 10^{-4}\right)$ & $\left(10.51 \cdot 10^{6}, 9.67 \cdot 10^{-4}\right)$ \\
\hline$\left(a_{\mathrm{Tb}}, b_{\mathrm{Tb}}\right)$ & $(0.099,-0.400)$ & \\
$\left(a_{\mathrm{Te}}, b_{\mathrm{Te}}\right)$ & & $(0.211,-1 / 3)$ \\
\hline
\end{tabular}

Tabelle A.6: Werte für den Transitionsbeginn bzw. das Transitionsende anhand der StMessungen für die Plattenposition A. Zusätzlich sind die bestimmten Fitparameter zur Berechnung von $S t_{\mathrm{Tb}}$ und $S t_{\mathrm{Te}}$ aufgelistet.

\begin{tabular}{lcc}
\hline$R e_{1}\left[10^{6} \mathrm{~m}^{-1}\right]$ & $\left(R e_{\mathrm{Tb}}, S t_{\mathrm{Tb}}\right)$ & $\left(R e_{\mathrm{Te}}, S t_{\mathrm{Te}}\right)$ \\
\hline 8 & $(-,-)$ & $\left(5.25 \cdot 10^{6},-\right)$ \\
& & $\left(5.19 \cdot 10^{6},-\right)$ \\
14 & $(-,-)$ & $\left(7.39 \cdot 10^{6},-\right)$ \\
& & $\left(7.30 \cdot 10^{6},-\right)$ \\
19 & $(-,-)$ & $\left(9.12 \cdot 10^{6}, 9.41 \cdot 10^{-4}\right)$ \\
& & $\left(9.12 \cdot 10^{6}, 8.95 \cdot 10^{-4}\right)$ \\
24 & $(-,-)$ & $\left(9.87 \cdot 10^{6}, 9.10 \cdot 10^{-4}\right)$ \\
& & $\left(10.08 \cdot 10^{6}, 8.47 \cdot 10^{-4}\right)$ \\
$\left(a_{\mathrm{Tb}}, b_{\mathrm{Tb}}\right)$ & $(-,-)$ & $(0.196,-1 / 3)$ \\
$\left(a_{\mathrm{Te}}, b_{\mathrm{Te}}\right)$ & & $(0.185,-1 / 3)$ \\
& &
\end{tabular}

Tabelle A.7: Werte für das Transitionsende anhand der St-Verteilung für die Plattenposition B. Da für den stromabgelegenen Messbereich zwei Kurven vorliegen, gibt es zum Transitionsende jeweils zwei Punkte. Zusätzlich sind die bestimmten Fitparameter zur Berechnung von $S t_{\mathrm{Te}}$ aufgelistet. Die Stellen des Transitionsbeginns konnten bei dieser Plattenposition nicht aus den StVerläufen bestimmt werden, da der Verlauf der Wärmestromdichte durch Störungen von der Lavaldüse beeinflusst ist. 



\section{Literaturverzeichnis}

Abu-Ghannam, B. J. \& Shaw, R. (1980), 'Natural transition of boundary layers-the effects of turbulence, pressure gradient, and flow history', Journal of Mechanical Engineering Science 22(5), 213-228.

Babinsky, H. \& Harvey, J. K. (2011), Shock wave-boundary-layer interactions, Cambridge University Press.

Bailly, C. \& Comte-Bellot, G. (2015), Turbulence, Springer International Publishing.

Benard, E., Cooper, R. K. \& Sidorenko, A. (2006), 'Transitional and turbulent heat transfer of swept cylinder attachment line in hypersonic flow', International journal of heat and mass transfer 49(5-6), 836-843.

Benay, R., Chanetz, B., Mangin, B., Vandomme, L. \& Perraud, J. (2006), 'Shock wave/transitional boundary-layer interactions in hypersonic flow', AIAA Journal 44(6), 12431254 .

Beresh, S. J., Henfling, J. F., Spillers, R. W. \& Pruett, B. O. M. (2011), 'Fluctuating wall pressures measured beneath a supersonic turbulent boundary layer', Physics of Fluids 23.

Birch, B., Buttsworth, D. \& Zander, F. (2020), 'Measurements of freestream density fluctuations in a hypersonic wind tunnel', Experiments in Fluids 61(158).

Brinich, P. F. (1956), Effects of leading-edge geometry on boundary layer transition at mach 3.1, Technical report, NACA TN 3659.

Canepa, E. S., Ubaldi, M. \& Zunino, P. (2002), Experiences in the application of intermittency detection techniques to hot-film signals in transitional boundary layers, in 'The 16th symposium on measuring techniques in transonic and supersonic flow in cascades and turbomachines'.

Canny, J. (1986), 'A computational approach to edge detection', IEEE Transactions on Pattern Analysis and Machine Intelligence PAMI-8(6), 679-698.

Cebeci, T. \& Bradshaw, P. (1984), Physical and Computational Aspects of Convective Heat Transfer, 1 ed., Springer-Verlag Berlin Heidelberg.

Chapman, D. R., Kuehn, D. M. \& Larson, H. K. (1957), Investigation of separated flows in supersonic and subsonic streams with emphasis on the effect of transition, Technical report, NACA TN 3869.

Corcos, G. M. (1963), 'Resolution of pressure in turbulence', The Journal of the Acoustical Society of America 35(2), 192-199. 
Currao, G. M. D., Choudhury, R., Gai, S. L., Neely, A. J. \& Buttsworth, D. R. (2020), 'Hypersonic transitional shock-wave-boundary-layer interaction on a flat plate', AIAA Journal $\mathbf{5 8}(2), 814-829$.

Deem, R. E. \& Murphy, J. S. (1965), 'Flat plate boundary layer transition at hypersonic speeds', AIAA 1965-128, 2nd Aerospace Sciences Meeting .

Délery, J. \& Marvin, J. G. (1986), 'Shock-wave boundary layer interactions', AGARDograph No. 280 .

Demetriades, A. (1977), Laminar boundary layer stability measurements at mach 7 including wall temperature effects, Technical report, Ford Aerospace and Communications Corp (AFOSR-TR-77-1311).

Demtröder, W. (2009), Experimentalphysik 2, 5 ed., Springer-Verlag Berlin Heidelberg.

Dhawan, S. \& Narasimha, R. (1958), 'Some properties of boundary layer flow during the transition from laminar to turbulent motion', Journal of Fluid Mechanics 3(4), 418-436.

Dolling, D. S. (2001), 'Fifty years of shock-wave/boundary-layer interaction research: what next?', AIAA Journal 39(8), 1517-1531.

Fedorov, A. (2011), 'Transition and stability of high-speed boundary layers', Annual Review of Fluid Mechanics 43(1), 79-95.

Fulghum, M. R. (2014), Turbulence measurements in high-speed wind tunnels using focusing laser differential interferometry, Phd thesis, Pennsylvania State University.

Gaitonde, D. V. (2015), 'Progress in shock wave/boundary layer interactions', Progress in Aerospace Sciences 72, 80-99.

Ginoux, J. J. (1969), On some properties of reattaching laminar and transitional high speed flows, Technical report, von Karman Institute for Fluid Dynamics Technical Note 53.

Gladstone, J. H. \& Dale, T. P. (1863), 'XIV. researches on the refraction, dispersion, and sensitiveness of liquids', Philosophical Transactions of the Royal Society of London 153, 317343.

Heffner, K., Chpoun, A. \& Lengrand, J. (1993), 'Experimental study of transitional axisymmetric shock-boundary layer interactions at Mach 5', AIAA 1993-3161, 23rd Fluid Dynamics, Plasmadynamics, and Lasers Conference .

Helm, C. M., Martín, M. P. \& Williams, O. J. H. (2021), 'Characterization of the shear layer in separated shock/turbulent boundary layer interactions', Journal of Fluid Mechanics $912(\mathrm{~A} 7)$.

Henderson, L. F. (1967), 'The reflexion of a shock wave at a rigid wall in the presence of a boundary layer', Journal of Fluid Mechanics 30(4), 699-722.

Hirschel, E. H. (2005), Basics of Aerothermodynamics, Springer-Verlag Berlin Heidelberg. 
Holden, M. S. (1977), 'Shock wave-turbulent boundary layer interaction in hypersonic flow', AIAA 1977-45, 15th Aerospace Sciences Meeting.

Houpt, A. W. \& Leonov, S. B. (2018), 'Focused laser differential interferometer for supersonic boundary layer measurements on flat plate geometries', AIAA 2018-3434, 2018 Plasmadynamics and Lasers Conference.

Houpt, A. W. \& Leonov, S. B. (2019), 'Focused and cylindrical-focused laser differential interferometer characterization of SBR-50 at Mach 2', AIAA 2019-3383, AIAA Aviation 2019 Forum .

Hung, F. T. \& Barnett, D. O. (1973), 'Shockwave-boundary layer interference heating analysis', AIAA 1973-237, 11th Aerospace Sciences Meeting .

Jewell, J. S., Hameed, A., Parziale, N. J. \& Gogineni, S. (2019), 'Disturbance speed measurements in a circular jet via double focused laser differential interferometry', AIAA 2019-2293, AIAA Scitech 2019 Forum .

Katzer, E. (1989), 'On the lengthscales of laminar shock/boundary-layer interaction', Journal of Fluid Mechanics 206, 477-496.

Knight, D. D. \& Degrez, G. (1998), 'Shock wave boundary layer interactions in high mach number flows - a critical survey of current numerical prediction capabilities', AGARD AR319, Volume 2.

Knight, D. D. \& Mortazavi, M. (2017), 'Hypersonic shock wave transitional boundary layer interactions - a review', AIAA 2017-3124, 47th AIAA Fluid Dynamics Conference.

Krishnan, L. \& Sandham, N. D. (2007), 'Strong interaction of a turbulent spot with a shockinduced separation bubble', Physics of Fluids 19.

Krogmann, P. (1995), The ludwieg tube tunnel B (RWG), Technical report, DLR IB 223-95 C30.

Kuehl, J. J. (2018), 'Thermoacoustic interpretation of second-mode instability', AIAA Journal 56(9), 3585-3592.

Lawson, J. M., Neet, M. C., Grossman, I. J. \& Austin, J. M. (2020), 'Static and dynamic characterization of a focused laser differential interferometer', Experiments in Fluids 61(187).

Lohmeyer, W. Q. \& DeJarnette, F. R. (2012), 'Transition from laminar to turbulent boundary layers', Journal of Spacecraft and Rockets 49(4), 766-768.

Longo, J. M. A. (2003), 'Aerothermodynamics - a critical review at DLR', Aerospace Science and Technology 7(6), 429-438.

Ludwieg, H. (1955), 'Der Rohrwindkanal', Zeitschrift für Flugwissenschaften 3(7), 206-216.

Ludwieg, H., Hottner, T. \& Grauer-Carstensen, H. (1969), 'Der Rohrwindkanal der Aerodynamischen Versuchsanstalt Göttingen', Jahrbuch 1969 der DGLR pp. 52-58. 
Lunte, J. \& Schülein, E. (2019), 'Heat transfer amplifications in transitional shockwave/boundary-layer interactions', AIAA 2019-3440, AIAA Aviation 2019 Forum .

Mack, L. M. (1984), 'Boundary-layer linear stability theory', AGARD Report No. 709, Special Course on Stability and Transition of Laminar Flow .

Mack, L. M. (1990), 'On the inviscid acoustic-mode instability of supersonic shear flows', Theoretical and Computational Fluid Dynamics 2, 97-123.

Mallinson, S. G., Gai, S. L. \& Mudford, N. R. (1996), 'Upstream influence and peak heating in hypervelocity shock wave/boundary-layer interaction', Journal of Propulsion and Power 12(5), 984-990.

Mertins, A. (2013), Signaltheorie, 3 ed., Springer Vieweg.

Meyer, E. \& Neumann, E.-G. (1974), Physikalische und Technische Akustik, 2 ed., Vieweg+Teubner Verlag.

Meyer, M. (2017), Signalverarbeitung, 8 ed., Springer Vieweg.

Morkovin, M. V. (1969), Critical evaluation of transition from laminar to turbulent shear layers with emphasis on hypersonically traveling bodies, Technical report, Air Force Flight Dynamics Laboratory AFFDL-TR-68-149.

Morkovin, M. V. (1987), Transition at hypersonic speeds, Technical report, ICASE Interim Report 1, NASA Contractor Rep. 178315.

Narasimha, R. (1985), 'The laminar-turbulent transition zone in the boundary layer', Progress in Aerospace Sciences 22(1), 29-80.

Narasimha, R. \& Dey, J. (1989), 'Transition-zone models for 2-dimensional boundary layers: A review', Sadhana 14, 93-120.

Needham, D. A. (1965), Laminar separation in hypersonic flow, $\mathrm{PhD}$ thesis, University of London.

Needham, D. \& Stollery, J. (1966), 'Boundary layer separation in hypersonic flow', AIAA 1966-455, 4th Aerospace Sciences Meeting.

Oertel, H. (2017), Prandtl - Führer durch die Strömungslehre, 14 ed., Springer Vieweg.

Ort, D. \& Dosch, J. J. (2019), 'Influence of mounting on the accuracy of piezoelectric pressure measurements for hypersonic boundary layer transition', AIAA 2019-2292, AIAA Scitech 2019 Forum .

Pagella, A., Rist, U. \& Wagner, S. (2002), 'Numerical investigations of small-amplitude disturbances in a boundary layer with impinging shock wave at Ma=4.8', Physics of Fluids 14(7), 2088-2101.

Parziale, N. (2013), Slender-body hypervelocity boundary-layer instability, Phd thesis, California Institute of Technology. 
Parziale, N. J., Shepherd, J. E. \& Hornung, H. G. (2012), 'Reflected shock tunnel noise measurement by focused differential interferometry', AIAA 2012-3261, 42nd AIAA Fluid Dynamics Conference and Exhibit.

Parziale, N. J., Shepherd, J. E. \& Hornung, H. G. (2013), 'Differential interferometric measurement of instability at two points in a hypervelocity boundary layer', AIA A 2013-521, 51st AIAA Aerospace Sciences Meeting including the New Horizons Forum and Aerospace Exposition .

Parziale, N. J., Shepherd, J. E. \& Hornung, H. G. (2014), 'Free-stream density perturbations in a reflected-shock tunnel', Experiments in Fluids $\mathbf{5 5}$.

Pate, S. R. \& Schueler, C. J. (1969), 'Radiated aerodynamic noise effects on boundary-layer transition in supersonic and hypersonic wind tunnels.', AIAA Journal 7(3), 450-457.

Potter, J. L. \& Whitfield, J. D. (1960), Effects of unit reynolds number, nose bluntness, and roughness on boundary layer transition, Technical report, Advisory group for aeronautical research and development - Report 256.

Ramprakash, A., McIntyre, T., Wheatley, V. \& Mee, D. (2019), 'Performance analysis of FLDI technique using turbulent jets', IX Australian Conference on Laser Diagnostics, Adelaide .

Reed, H. L., Saric, W. S. \& Arnal, D. (1996), 'Linear stability theory applied to boundary layers', Annual Review of Fluid Mechanics 28, 389-428.

Reshotko, E. (1976), 'Boundary-layer stability and transition', Annual Review of Fluid Mechanics 8, 311-349.

Ross, R. (1972), 'A simple formula for flat plate boundary-layer transition in supersonic wind tunnels', AIAA Journal 10(3), 336-337.

Saric, W. S., Reed, H. L. \& Kerschen, E. J. (2002), 'Boundary-layer receptivity to freestream disturbances', Annual Review of Fluid Mechanics 34, 291-319.

Schlichting, H. \& Gersten, K. (2006), Grenzschicht-Theorie, 10 ed., Springer-Verlag Berlin Heidelberg.

Schmidt, B. E. \& Shepherd, J. E. (2015), 'Analysis of focused laser differential interferometry', Applied Optics 54(28).

Schneider, S. P. (1995), 'Improved methods for measuring laminar-turbulent intermittency in boundary layers', Experiments in Fluids 18, 370-375.

Schneider, S. P. (2008a), 'Development of hypersonic quiet tunnels', Journal of Spacecraft and Rockets 45(4), 641-664.

Schneider, S. P. (2008b), 'Effects of roughness on hypersonic boundary-layer transition', Journal of Spacecraft and Rockets 45(2), 193-209. 
Schneider, S. P. (2015), 'Developing mechanism-based methods for estimating hypersonic boundary-layer transition in flight: The role of quiet tunnels', Progress in Aerospace Sciences $\mathbf{7 2}, 17-29$.

Schneider, S. P. (2019), 'Towards a process for calibrating 1-MHz pressure-fluctuation sensors with small pressure steps in a shock tube', Final Report for AFOSR Grant FA9550-16-10407, AFRL-AFOSR-VA-TR-2019-0362 .

Schopper, M. R. (1984), 'Interaction of aerodynamic noise with laminar boundary layers in supersonic wind tunnels', NASA Contractor Report 3621.

Schülein, E. (1999), Qualität der Strömung nach der Nachbearbeitung der Überschalldüse „M $=3$ “ des Rohrwindkanals des DLR in Göttingen, Technical report, DLR IB 223-99 A 12.

Schülein, E. (2006), 'Skin friction and heat flux measurements in shock/boundary layer interaction flows', AIAA Journal 44(8), 1732-1741.

Schülein, E. (2014), 'Effects of laminar-turbulent transition on the shock-wave/boundary-layer interaction', AIAA 2014-3332, 44th AIAA Fluid Dynamics Conference.

Schülein, E. \& Wagner, A. (2015), Freestream disturbance characterization in DNW-RWG at Mach 3 and 6, Technical report, DLR-224-2014 C 169.

Schultz, D. L. \& Jones, T. V. (1973), 'Heat-transfer measurements in short-duration hypersonic facilities', AGARDograph No. 165.

Schwamborn, D., Gerhold, T. \& Heinrich, R. (2006), 'The DLR TAU-code: Recent applications in research and industry', European Conference on Computational Fluid Dynamics .

Settles, G. S. (2001), Schlieren and Shadowgraph Techniques, 1 ed., Springer-Verlag Berlin Heidelberg.

Settles, G. S. \& Fulghum, M. R. (2016), 'The focusing laser differential interferometer, an instrument for localized turbulence measurements in refractive flows', Journal of Fluids Engineering 138.

Simeonides, G. A. (2003), 'Correlation of laminar-turbulent transition data over flat plates in supersonic/hypersonic flow including leading edge bluntness effects', Shock Waves 12, 497508.

Simeonides, G., Haase, W. \& Manna, M. (1994), 'Experimental, analytical, and computational methods applied to hypersonic compression ramp flows', AIAA Journal 32(2), 301-310.

Smeets, G. \& George, A. (1973), Laser-differential interferometer applications in gas dynamics, Technical report, Institut Saint-Louis Report 28/73, translated 1996 by A. Goetz.

Solomon, Jr., O. M. (1991), PSD computations using welch's method., Technical report, Sandia Report SAND91-1533, Sandia National Laboratories.

Souverein, L., Bakker, P. \& Dupont, P. (2013), 'A scaling analysis for turbulent shockwave/boundary-layer interactions', Journal of Fluid Mechanics 714, 505-535. 
Spalding, D. B. \& Chi, S. W. (1964), 'The drag of a compressible turbulent boundary layer on a smooth flat plate with and without heat transfer', Journal of Fluid Mechanics 18(1), 117143.

Stetson, K. F., Thompson, E. R., Donaldson, J. C. \& Siler, L. G. (1989), 'Laminar boundary layer stability experiments on a cone at Mach 8, part 5 - tests with a cooled model', AIAA 1989-1895, 20th Fluid Dynamics, Plasma Dynamics and Lasers Conference.

Stetson, K. \& Kimmel, R. (1992), 'On hypersonic boundary-layer stability', AIAA 1992-737, 30th Aerospace Sciences Meeting and Exhibit.

Tomasi, C. \& Manduchi, R. (1998), 'Bilateral filtering for gray and color images', IEEE Sixth International Conference on Computer Vision pp. 839-846.

Torrence, C. \& Compo, G. P. (1998), 'A practical guide to wavelet analysis', Bulletin of the American Meteorological Society 79(1), 61-78.

Van Driest, E. R. (1956), 'The problem of aerodynamic heating', Aeronautical Engineering Review pp. 21-41.

Veerasamy, D. \& Atkin, C. (2020), 'A rational method for determining intermittency in the transitional boundary layer', Experiments in Fluids 61(11).

Waindim, M., Agostini, L., Larchêveque, L., Adler, M. \& Gaitonde, D. V. (2020), 'Dynamics of separation bubble dilation and collapse in shock wave/turbulent boundary layer interactions', Shock Waves 30, 63-75.

Weisberger, J. M., Bathel, B. F., Herring, G. C., King, R. A., Chou, A. \& Jones, S. B. (2019), 'Two-point focused laser differential interferometry second-mode measurements at mach 6', AIAA 2019-2903, AIAA Aviation 2019 Forum .

Welch, P. (1967), 'The use of fast fourier transform for the estimation of power spectra: a method based on time averaging over short, modified periodograms', IEEE Transactions on Audio and Electroacoustics 15(2), 70-73.

White, F. (1991), Viscous fluid flow, 2 ed., McGraw-Hill.

Willems, S. \& Gülhan, A. (2013), 'Experiments on shock induced laminar-turbulent transition on a flat plate at Mach 6', 5th European Conference for Aeronautics and Space Sciences (EUCASS) .

Willems, S., Gülhan, A. \& Steelant, J. (2015), 'Experiments on the effect of laminar-turbulent transition on the SWBLI in H2K at Mach 6', Experiments in Fluids 56(49).

Zhong, X. \& Wang, X. (2012), 'Direct numerical simulation on the receptivity, instability, and transition of hypersonic boundary layers', Annual Review of Fluid Mechanics 44, 527-561. 
Literaturverzeichnis

132 


\section{Lebenslauf}

\section{Persönliche Daten}

Name:

Jens Bernard Lunte

Geburtsdatum:

24.08.1992

Geburtsort:

Lohne

Familienstand:

ledig

Staatsangehörigkeit:

deutsch

\section{Ausbildung und Studium}

2011

2014

2016

seit 2016

Abitur am Gymnasium Lohne

Bachelor of Science in Physik an der

Georg-August-Universität Göttingen

Master of Science in Physik an der

Georg-August-Universität Göttingen

Promotionsstudiengang Physik an der

Georg-August-Universität Göttingen

\section{Berufserfahrung}

seit 2016

Doktorand und wissenschaftlicher Mitarbeiter am Deutschen

Zentrum für Luft- und Raumfahrt e.V., Göttingen 